\title{
Image features for the future in stage IV non-small cell lung cancer
}

Citation for published version (APA):

de Jong, E. (2019). Image features for the future in stage IV non-small cell lung cancer. [Doctoral Thesis, Maastricht University]. Ipskamp Printing BV. https://doi.org/10.26481/dis.20190206ej

Document status and date:

Published: 01/01/2019

DOI:

10.26481/dis.20190206ej

Document Version:

Publisher's PDF, also known as Version of record

\section{Please check the document version of this publication:}

- A submitted manuscript is the version of the article upon submission and before peer-review. There can be important differences between the submitted version and the official published version of record.

People interested in the research are advised to contact the author for the final version of the publication, or visit the DOI to the publisher's website.

- The final author version and the galley proof are versions of the publication after peer review.

- The final published version features the final layout of the paper including the volume, issue and page numbers.

Link to publication

\footnotetext{
General rights rights.

- You may freely distribute the URL identifying the publication in the public portal. please follow below link for the End User Agreement:

www.umlib.nl/taverne-license

Take down policy

If you believe that this document breaches copyright please contact us at:

repository@maastrichtuniversity.nl

providing details and we will investigate your claim.
}

Copyright and moral rights for the publications made accessible in the public portal are retained by the authors and/or other copyright owners and it is a condition of accessing publications that users recognise and abide by the legal requirements associated with these

- Users may download and print one copy of any publication from the public portal for the purpose of private study or research.

- You may not further distribute the material or use it for any profit-making activity or commercial gain

If the publication is distributed under the terms of Article $25 \mathrm{fa}$ of the Dutch Copyright Act, indicated by the "Taverne" license above, 


\section{Image features for the future in stage IV non-small cell lung cancer}




\section{Cover}

The cover shows a voxel representation of a tumor imaged using CT and using PET/CT. The original CT and PET/CT image of the tumor is shown at the back. In the lung, names of image features are shown.

Production Typesetting: This thesis was created using ATEX

Printing: Ipskamp Printing BV, Enschede

ISBN: 978-94-028-1347-0

\section{Copyrights}

(C) Copyright E.E.C. de Jong, Maastricht 2019

No parts of this thesis publication may be reproduced in any form without prior written permission of the holder of the copyrights. 


\title{
Image features for the future in stage IV non-small cell lung cancer
}

\author{
Proefschrift \\ ter verkrijging van de graad van doctor aan de Universiteit Maastricht, \\ op gezag van de Rector Magnificus Prof. dr. Rianne M. Letschert, \\ volgens het besluit van het College van Decanen, \\ in openbaar te verdedigen \\ op woensdag 6 februari 2019 om 14:00 uur \\ door
}

Evelyn Elizabeth Catharina de Jong 


\section{Promotors}

Prof. dr. P. Lambin

Prof. dr. A.-M.C. Dingemans

\section{Co-promotor \\ Dr. ir. W. van Elmpt}

\section{Beoordelingscommissie}

Prof. dr. F.M. Mottaghy (voorzitter)

Prof. dr. J.G.J.V. Aerts (Erasmus Medisch Centrum)

Prof. dr. W.H. Backes

Prof. dr. M. van den Heuvel (Radboud Universitair Medisch Centrum)

Dr. A. Hoeben 


\section{Contents}

\section{General introduction}

Chapter 1 Introduction and outline of the thesis

Chapter 2 Radiomics applied to lung cancer: a review

\section{Methodological challenges in image feature studies}

Chapter 3 Quality assessment of positron emission tomography scans: recommendations for future multicenter trials

Chapter 4 What you see is (not) what you get: tools for a non-radiologist to evaluate image quality in lung cancer

Chapter 5 Influence of gray level discretization on radiomic feature stability for different CT scanners, tube currents and slice thicknesses: a comprehensive phantom study

\section{Image features for prognosis and cachexia}

Chapter $6 \quad{ }^{18} \mathrm{~F}$-FDG PET/CT-based response assessment of stage IV non-small cell lung cancer treated with paclitaxel-carboplatin-bevacizumab with or without nitroglycerin patches

Chapter 7 Applicability of a prognostic CT-based radiomic signature model trained on stage I-III non-small cell lung cancer in stage IV non-small cell lung cancer

Chapter 8 Can radiomic features describe lung semantic features in non-small cell lung cancer patients?

Chapter 9 Radiomics approach to predict skeletal muscle response to chemotherapy in stage IV non-small cell lung cancer

\section{Discussion}

Chapter 10 General discussion and future perspectives 


\section{Appendices}

$\begin{array}{ll}\text { Summary } & 255\end{array}$

$\begin{array}{ll}\text { Samenvatting } & 261\end{array}$

$\begin{array}{ll}\text { Valorisation addendum } & 267\end{array}$

$\begin{array}{ll}\text { Dankwoord } & 271\end{array}$

$\begin{array}{ll}\text { Curriculum vitae } & 277\end{array}$ 


General introduction 

Chapter 1

Introduction and outline of the thesis 


\section{Lung Cancer}

\section{Incidence and mortality}

In the Netherlands, each year more than 100,000 people are diagnosed with cancer, and while many of them are cured, still about 40,000 patients yearly die. In the Netherlands in 2016, 6291 men and 4383 women died due to lung cancer, which is about $24 \%$ of all cancer deaths in the Netherlands. Lung cancer is the type of cancer with the highest mortality in the Netherlands [1]. Lung cancer can be divided into non-small cell lung cancer (NSCLC) and small cell lung cancer (SCLC). Of all lung cancers about $80 \%$ is specified as NSCLC. NSCLC can be subdivided into squamous cell carcinoma, adenocarcinoma and large cell carcinoma. The biggest risk factor in lung cancer is smoking, about $71 \%$ of the 1.59 million deaths yearly is due to lung cancer caused by smoking. In Asian countries, an increase in lung cancer diagnosis in non-smokers is observed, which is associated with specific molecular and genetic tumor characteristics.

\section{Molecular subtypes}

Examples of molecular tumor subtypes of NSCLC are epidermal growth factor (EGFR) mutated tumors, tumors with mutations in v-Ki-ras2 Kirsten rat sarcoma (KRAS) and tumors with a rearrangement in the anaplastic lymphoma kinase (ALK) [2]. About 10-12 \% of the Caucasians with adenocarcinoma have an EGFR mutation. EGFR mutations are more frequent in females, never smokers and East Asian patients. Almost 15-25\% of the NSCLC patients have a KRAS mutation, which occur more frequently in adenocarcinomas, Caucasian patients and smokers [2]. ALK rearrangements, which are present in about $5 \%$ of the adenocarcinomas, are more frequent seen in never smokers and younger patients. Testing for EGFR mutations and ALK rearrangements is recommended in all patients with advanced non-squamous cell carcinoma [3].

\section{Staging of non-small cell lung cancer}

The overall survival rate in lung cancer is low, on average the 5-years survival rate is $16 \%$ [4] and is dependent on the stage of the disease. NSCLC is staged according to the Tumor-Node-Metastasis classification of the American Joint Committee on Cancer (AJCC) and grouped into eight stages (Stage 0, IA, IB, IIA, IIB, IIIA, IIIB and IV). Patients with distant metastases are classified as stage IV patients independent of the T- and N-stage of the tumor [5]. The 5-years survival rate in stage I NSCLC is $61 \%$, stage II $43 \%$, stage III $18 \%$ and it decreased to only $3 \%$ in stage IV [6]. The extent of the primary tumor is determined using computed tomography (CT) imaging, and the assessment of lymph node involvement and metastases is performed using positron emission tomography (PET) imaging. In advanced stage patients, it is recommended to image the brain, preferably by magnetic resonance imaging $(\mathrm{MRI})$ with gadolinium enhancement or by $\mathrm{CT}$ with iodine contrast.

\section{Treatment of non-small cell lung cancer}

Treatment of lung cancer is different for the various stages of lung cancer. But next to the tumor stage, other clinical variables like smoking history, performance status, age, molecular pathology, age, co-morbidities and patient preferences are taken into account 
to determine the optimal treatment for the patient. In all stages of NSCLC, patients should be motivated to quit smoking, because smoking may interact with systemic therapy [3]. About $40 \%$ of the NSCLC patients are diagnosed with stage IV disease. Systemic therapy is advised in all stage IV patients with a good performance status. Chemotherapy for stage IV patients, at the time of the studies described in this thesis, consisted of platinum doublets [3]. The drugs most often used in NSCLC are cisplatin, carboplatin, paclitaxel, docetaxel, gemcitabine, pemetrexed. Many clinical trials are performed to improve outcome and quality of life of patients and individualize treatment in the future. An example, described in this thesis, is the Dutch randomized phase II trial, the NVALT12, in which the effect of the addition of nitroglycerin, to paclitaxel-carboplatin-bevacizumab therapy, on progression free survival, was investigated [7]. Carboplatin is in the platinumbased antineoplastic family and works by interfering with duplication of DNA [8]. Paclitaxel is in the taxane family and works by interference with the normal function of microtubules during cell division [9]. Tumor hypoxia is a poor prognostic factor in lung cancer. Hypoxia Inducible Factor (HIF-1 $\alpha$ ) is the major factor regulating the response to hypoxia. HIF directly activates the vascular endothelial growth factor which helps the tumor to grow. Hypoxia is related to treatment resistance. Hypoxic cells are more radio-resistant, more chemo-resistant and more prone to develop distant metastases than normoxic cells [10]. A phase III study showed that the addition of bevacizumab to paclitaxel-carboplatin therapy improved overall survival, due to the blocking of the vascular endothelial growth factor by bevacizumab [11]. Bevacizumab is in the angiogenesis inhibitor and monoclonal antibody family and works by slowing the growth of new blood vessels [12]. Bevacizumab helps reducing hypoxia which might lead to a more efficient treatment. By adding nitroglycerin, which is a vasodilator [13], the blood flow the tumor was expected to increase, which could therefore also decrease hypoxia related drug resistance. However, the conclusion of the NVALT12 study was that by adding NTG the progression free survival as well as the overall survival did not improve [7].

In September 2018 the clinical practice guidelines for diagnosis, treatment and followup in metastatic NSCLC are updated [14]. Recent development of immune checkpoint inhibitors have shown that immunotherapy can play an important role in the treatment of stage IV NSCLC.

The preferred treatment of EGFR mutated and ALK rearranged NSCLC is with specific targeted tyrosine kinase inhibitors. By individualization of the treatment according to the molecular tumor characteristics a better progression free survival and overall survival is typically obtained.

\section{Prognostic and predictive models}

Although many different treatments are available for lung cancer patients, it is quite difficult to select patients for a specific treatment. Prognostic models exists of a combination of variables that estimate the probability of an outcome, for example survival, as good as possible, while predictive models can distinguish patients who can benefit from a specific treatment [15]. Prognostic and predictive models can be used to tailor treatment decisions and therefore, individualization of the balance of reduction of side effects and prognosis and quality of life of patients is optimized [16]. Clinical variables used in prognostic models in lung cancer are for example age, gender, smoking history, comorbidity, performance status, forced expiratory volume in 1 second, T-stage, $\mathrm{N}$-stage, M-stage, histological grading and mutation status. Another phenomenon 
related to a worse prognosis in lung cancer is cachexia. Cachexia describes involuntary weight loss in patients with cancer that cannot be reversed by normal nutritional support. A defining feature of cancer cachexia is loss of muscle, but fat may be lost as well. In addition to muscle wasting and loss of appetite, patients who suffer from cancer cachexia have a poor quality of life and experience pain and fatigue. About $61 \%$ of newly diagnosed NSCLC patients have weight loss. Patients with cachexia have a higher prevalence of toxic adverse effects to systemic treatment, while the dose of systemic treatment is specified for a certain body mass which decreases in cachectic patients. Cancer cachexia is a multidimensional syndrome and therefore a multimodal treatment is needed. The main driver of the cachexia is the tumor. All factors that have a negative impact on food intake and well-being need to be minimized. Inflammatory mediators possibly play a role in the development of cachexia [17].

In predictive models different treatment groups are the input variables. Prognostic models can also contain biological markers that can for example be measured in the blood, so called biomarkers. Image characteristics, like SUV metrics, but also CT intensity values can potentially improve prognostic and predictive models.

\section{Imaging in stage IV NSCLC}

\section{CT, PET and MRI}

Medical imaging is an important part of routine care in diagnosing, treatment and followup in oncology. For most lung cancer patients a contrast-enhanced CT of the chest and upper abdomen including complete assessment of liver, kidneys and adrenal glands is used for diagnosis and staging. CT is a diagnostic imaging procedure that uses $\mathrm{X}$-rays to build cross sectional images ("slices") of the body. Slices are reconstructed from attenuation measurements of the X-rays through the patient's body. The attenuation is measured from different angles to make transmission or projection images. These projection images are reconstructed into a (electron) density volumetric image expressed in Hounsfield units (HU).

PET is a molecular imaging technique that is used in staging, because it offers the highest sensitivity for tumor infiltration of mediastinal lymph nodes and distant metastases assessment [18]. Depending on tracer characteristics specific biological processes can be monitored. The most frequently used tracer in oncology is 2-deoxy-2-fluorine-18-fluoro-D-glucose $\left({ }^{18} \mathrm{~F}-\mathrm{FDG}\right)$, which is an analogue of glucose. Unlike glucose, when ${ }^{18} \mathrm{~F}-\mathrm{FDG}$ is taken up into the cell, it is converted to ${ }^{18}$ F-FDG-6-phosphate, which cannot undergo further metabolism and is therefore trapped in the cell. An increased cellular uptake of ${ }^{18} \mathrm{~F}-\mathrm{FDG}$ and a higher rate of intracellular phosphorylation lead to higher uptakes and trapping of FDG in cancer cells [19]. The FDG is labeled with ${ }^{18} \mathrm{~F}$, which is a radioactive isotope of fluorine that emits a positron during radioactive decay, which annihilates with a nearby electron to emit two $511 \mathrm{keV}$ photons in almost opposite directions. These two annihilations photons are registered by the PET scanner using a coincidence detection principle, providing 3D information on the tracer distribution. Nowadays, the PET and CT are often combined in one imaging device, the PET/CT scanner. This has the advantage that the patient is in exactly the same position for the CT as for the PET scan, which makes the fusion of the PET scan with the CT scan easier. Using the attenuation properties of the patient derived from the $\mathrm{CT}$, a higher quantitative accuracy is achieved by taking into account 
the patient self-attenuation. However, accurate quantification of tracer uptake remains difficult as many processes influence the PET image. The measured concentration of radioactivity in tissue depends on the tracer concentration in blood over time, which in turn depends on the injected dose and distribution volume. The standardized uptake value (SUV) is corrected for those variabilities. Next to that, the PET signal does not necessarily reflect specific uptake, e.g. trapping of the tracer by the process of interest. Pharmacokinetic modelling can be applied to distinguish between the various kinetic processes and separates the total signal into the uptake in blood, uptake free in tissue, specific uptake (tumor) and non-specific uptake [20]. Figure 1.1 shows an example of a PET/CT scan. In advanced NSCLC PET/CT is the used in the detection of bone metastasis, the evaluation of solitary pulmonary nodules, intra-thoracic pathological lymph nodes and distant metastasis [14].

MRI may complement or improve diagnostic accuracy, mainly in assessing local chest wall, vascular or vertebra invasion. MRI is also effective for identification of nodal and distant metastases. MRI is more sensitive than CT in detecting brain metastasis [14]. In MRI a strong magnetic field is produced by a powerful magnet that forces the protons in the body to align with the field. A radiofrequency pulse let the magnetic vector of the protons deflect. When the radiofrequency pulse is switched off the protons start to align again with the magnetic field. The energy released by the protons during alignment as well as the time it takes is measured and is different for different tissues [21].

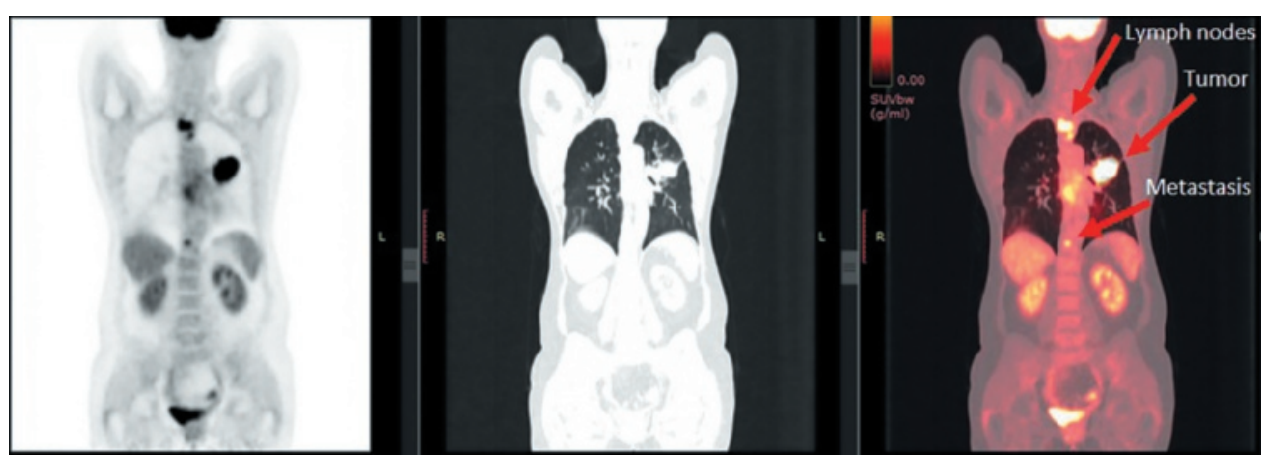

Figure 1.1: Left image shows an ${ }^{18}$ F-FDG-PET scan of a patient with stage IV non-small cell lung cancer, with a tumor in the left lung, positive lymph nodes in the top of the lung and a metastasis in the spine; the middle image shows the CT scan of the same patient and the right image shows the fusion of the PET and the CT scan.

\section{Image quality and standardization}

Due to technology innovations and the combination of PET and CT in one imaging device, the image quality of CT and PET scans has improved over the years. Although due to the many available image settings a large variability in image parameters exists between different hospitals. When comparing scans from different hospitals, it is therefore important to be aware of possible differences. To minimize differences the European Association of Nuclear Medicine (EANM) and some other initiatives provided guidelines for imaging $[22,23,24]$. The first initiative for standardization of PET imaging was the Netherlands protocol for standardization of FDG whole body PET studies in 
multi-center trials (NEDPAS) in 2008 [24]. The protocol is based on standardization of 1) patient preparation; 2) matching of scan statistics by prescribing dosage as function of patient weight, scan time per bed position, percentage of bed overlap and image acquisition mode; 3) matching of image resolution by prescribing reconstruction settings for each type of scanner; 4) matching of data analysis procedure by defining volume of interest methods and SUV calculations and 5) a multicenter quality control procedure for verification of scanner calibration and of activity concentration recoveries using the National Electrical Manufacturers Association (NEMA) image quality phantom. The protocol reduced variability of SUV across centers participating in multicenter trials. Quality control phantom experiments demonstrated that use of the protocol reduced calibration errors from up to $15 \%$ to within $6 \%$ [24]. In 2010 the NEDPAS guidelines were further extended in the EANM procedure guidelines for tumour imaging version 1.0 [22], which were updated in 2014 to the EANM procedure guidelines for tumour imaging version 2.0 [23]. The purpose of these guidelines is to assist physicians in recommending, performing, interpreting, and reporting the results of FDG PET/CT for oncological imaging of adult patients. PET is a quantitative imaging technique and therefore requires a common quality control assurance procedure to maintain the accuracy and precision of quantification. Repeatability and reproducibility are two essential requirements for any quantitative measurement. Repeatability relates to the uncertainty in obtaining the same result in the same patient when he or she is examined more than once on the same system. Reproducibility, is the ability to yield the same result in the same patient when that patient is examined on different systems and at different imaging sites. Adequate repeatability and reproducibility are essential for the clinical management of patients and the use of FDG PET/CT within multicenter trials. A common standardized imaging procedure will help promote the appropriate use of FDG PET/CT imaging and increase the value of publications and therefore, their contribution to evidence based medicine. Moreover, consistency in numerical values between platforms and institutes that acquire the data will potentially enhance the role of semi-quantitative and quantitative image interpretation. Precision and accuracy are additionally important as FDG PET/CT is used to evaluate tumor response as well as for diagnosis, prognosis and staging. Therefore, guidelines specifically aim to achieve standardized uptake value harmonization in multicenter settings [23].

For CT the guidelines are less pronounced. In some countries, for example in the United States, low dose CT scans are performed for lung cancer screening. CT guidelines are developed for those screenings, while for CT scans not performed during screening, protocols are not always standardized. The Alliance for Quality Computed Tomography (AQCT) working group provided information, to ensure that the minimum amount of radiation, to obtain the diagnostic information needed to answer the clinical question, is used. Reference protocols are developed for specific diagnostic tasks by the AQCT working group. The lung cancer screening protocol from the American Association of Physicists in Medicine (AAPM) specifies that the CT scan should be performed in breath hold, because tumor motion is problematic. Next to that, thin image slices should be used, slice thickness preferably less than $2.5 \mathrm{~mm}$. It is encouraged to reconstruct in the axial, but also in the sagittal and coronal direction. The dose should be adjusted to the patient size but should be around $3 \mathrm{mGy}$ [25]. The AAPM protocol for a routine adult chest-abdomen-pelvis CT states that contrast can be applied orally or intravenously, depending on the indication and the preference of the radiologist. The patient should be in supine position with the arms above the head, centered within the gantry for a proper functioning of the automatic 
exposure control system. The scan range is typically from the top of the lungs to either the iliac crest or the pubic symphysis. The slice thickness should be as small as possible, but is dependent on manufacturer [26].

\section{Image biomarkers}

Image characteristics used in prognostic and predictive models are called image biomarkers. An application for image biomarkers is the TNM-stage [27]. Image biomarkers can be qualitative, so called semantic features, or quantitative, for example radiomic features. Image biomarkers can reveal the tumor's phenotype, which represents the underlying biology of the tumor. To determine the tumor's phenotype, typically a biopsy was taken. However, a biopsy might be difficult to obtain, is invasive for the patient and is just a sample from a generally heterogeneous larger lesion. It has been shown that semantic features like, air bronchogram and pleural retraction are related to EGFR mutated tumors, while pleural effusion is related to ALK mutated tumors [28]. This shows that using imaging, mutation statuses, as EGFR, KRAS and ALK, can be distinguished and the invasive biopsy is in the future possibly superfluous. Semantic features are mostly visually scored by radiologists and describe a tumor's shape and internal structure [29]. Examples of semantic features in lung cancer are location of the lesion in the lung; shape (round, complex, oval); presence of ground-glass opacity and presence of cavitation. Figure 1.2 shows some examples of semantic features in NSCLC.

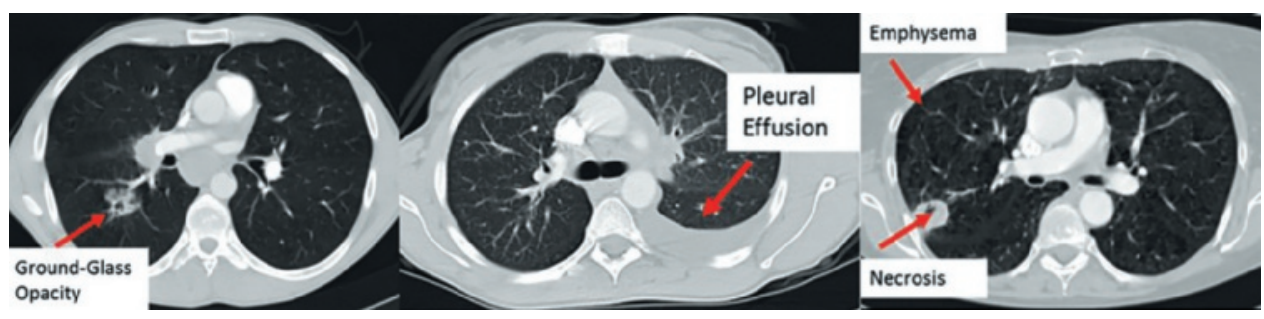

Figure 1.2: Examples of the semantic features: ground glass opacity, pleural effusion, emphysema and necrosis.

While semantic features are scored manually, radiomic features can be extracted automatically. These features describe the intensity distribution, spatial relationships between the various intensity levels, texture heterogeneity patterns, shape and the relation of the tumor with its surroundings The region of interest need to be segmented and then radiomic software can extract hundreds of features For radiomics, it is important that the images are of high quality and performed according to a standardized protocol. To select useful features for the specific research question a feature selection procedure need to be performed [30].

In 2014 a radiomic signature model is built in a cohort of stage I-III lung cancer patients and validated in an independent cohort of stage I-III lung cancer patients. This radiomic signature was surprisingly as well prognostic in head and neck cancer [31]. Figure 1.3 shows an example of radiomic features. 


\section{Objective and outline of the thesis}

The aim of this thesis is to investigate the use of image features in stage IV NSCLC and presents the challenges for using image features in prognostic and predictive models. The hypothesis is that simple and advanced qualitative and quantitative image features from standardized medical imaging help to improve patient care.

After an introduction, the thesis is divided in three parts. Part I, the introduction of radiomics in lung cancer, contains chapter 2, which is a comprehensive review on the multiple radiomics studies performed in lung cancer. This review summarizes the results of 3 PET radiomics papers and $8 \mathrm{CT}$ radiomics papers. The main applications of radiomics were the classification of lung nodules (diagnostic) and prognostication of lung cancer (theragnostic). Only 5 of the 22 studies were externally validated. It was clear that radiomics had great potential although some methodological issues need to be solved first which some are investigated in this thesis (see Chapter 5).

Part II describes the methodological challenges in image feature studies that have
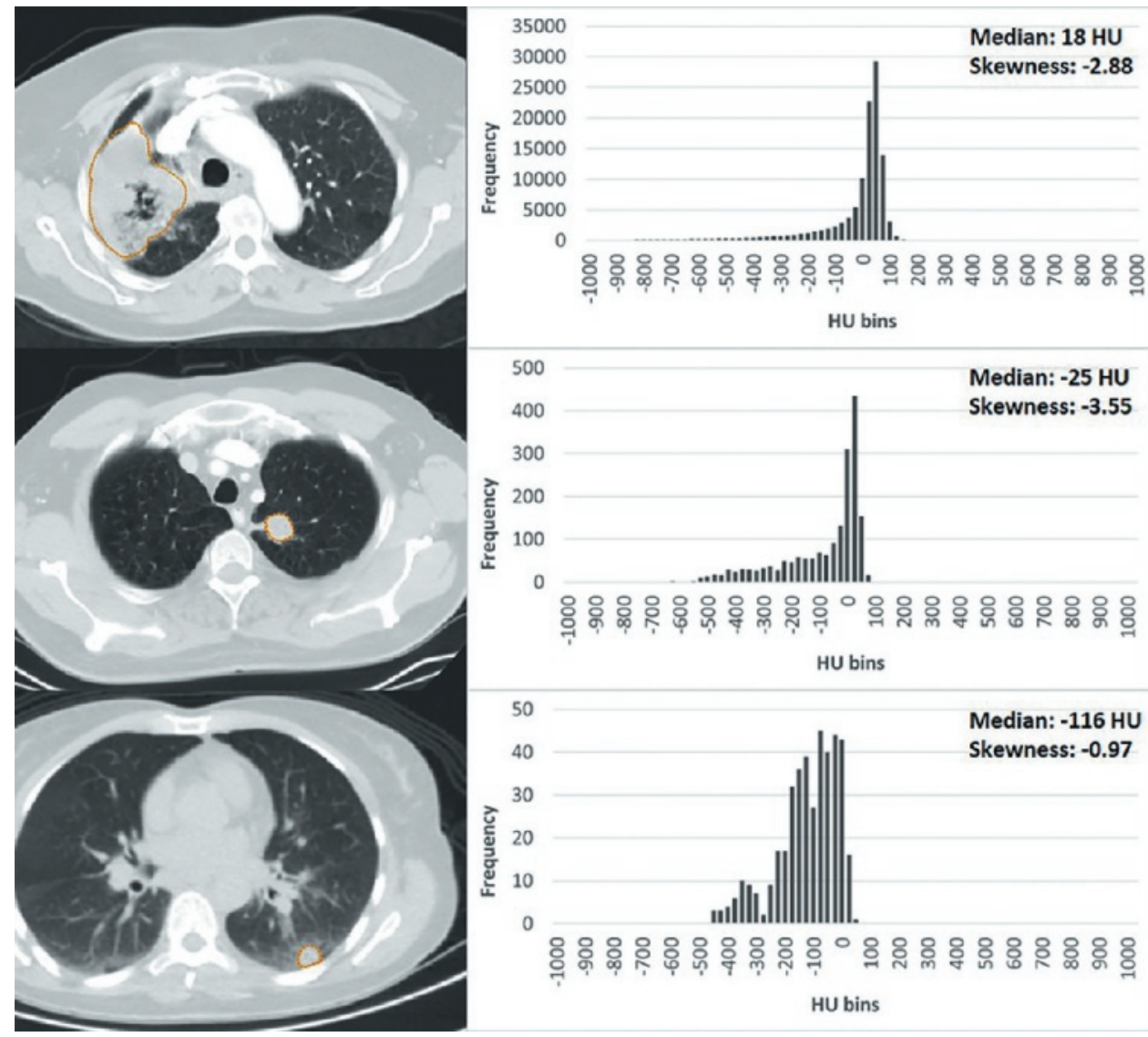

Figure 1.3: Examples of radiomic features. The left images show the delineated tumor on CT; on the right the intensity histogram of the tumor with an example of the radiomic features: median and skewness. 

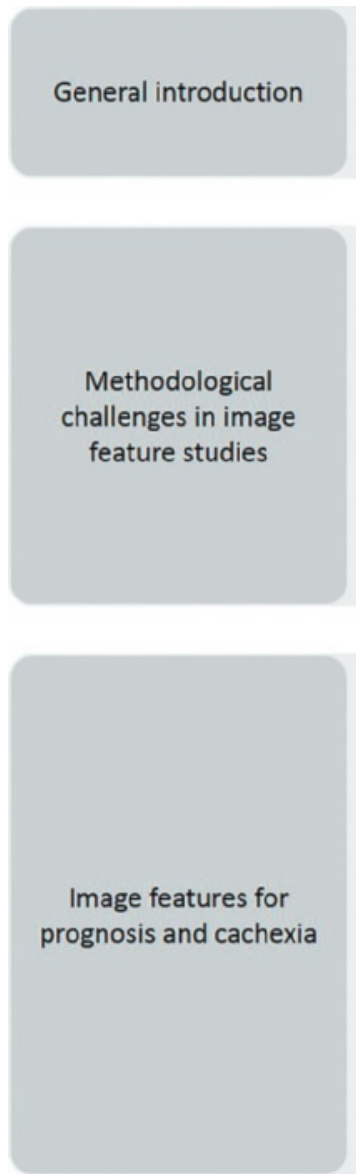

Discussion

\section{Chapter 1}

Introduction and outline of the thesis

Chapter 2

Radiomics applied to lung cancer: a review

\section{Chapter 3}

Quality assessment of positron emission tomography scans:

recommendations for future multicenter trials

\section{Chapter 4}

What you see is (not) what you get: How to evaluate image quality in lung cancer as a non-radiologist

\section{Chapter 5}

Influence of gray level discretization on radiomic feature stability for different CT scanners, tube currents and slice thicknesses: $a$ comprehensive phantom study

\section{Chapter 6}

[18F] FDG PET/CT-based response assessment of stage IV non-small cell lung cancer treated with paclitaxel-carboplatin-bevacizumab with or without nitroglycerin patches

\section{Chapter 7}

Applicability of a prognostic CT-based radiomic signature model trained on stage I-III non-small cell lung cancer in stage IV non-small cell lung cancer

\section{Chapter 8}

Can radiomic features describe lung semantic features in non-small cell lung cancer patients?

\section{Chapter 9}

Are radiomic features of skeletal muscle prognostic for overall survival?

\section{Chapter 10}

General discussion and future perspectives

Figure 1.4: Schematic outline of the thesis.

to be overcome in the future. In chapter 3 the challenges of PET response assessment in multicenter studies are described. Although standardization protocols and guidelines are available, an image sub-study of the NVALT12 trial, in which repeated PET images were performed, showed that there is still a large variability in image acquisition and reconstruction parameters. In this chapter the image parameters of the PET scans of the NVALT12 were compared to the image parameters recommended by the EANM.

Based on the results recommendations are proposed for future multicenter studies using PET for response assessment. Chapter 4 describes the problems with imaging in two clinical trials. Clinicians are not always aware of the technical details influencing image interpretation, and imaging experts are not always aware of the clinical aim of a particular scan. This chapter describes simple guidelines for clinicians to judge if a scan can be used to answer the clinical question. In chapter 5 the influence of different image parameters 
on radiomic features is reported. In this chapter a phantom study is done to investigate the influence of slice thickness, exposure, bin width and resampling methods on radiomic texture features.

Part III describes the use of simple and advanced qualitative and quantitative image features for the prediction of cachexia and treatment outcome (survival). In chapter 6 the use of simple quantitative PET features for prognosis prediction based on the NVALT12 trial was investigated. The chapter describes the investigation of the feasibility of ${ }^{18} \mathrm{~F}-$ FDG PET for response assessment to paclitaxel-carboplatin-bevacizumab treatment with and without NTG patches. A $30 \%$ decrease in CT and PET parameters was used to investigate response, which was correlated to overall survival. Chapter 7 describes the applicability of the radiomic signature trained on stage I-III NSCLC patients in stage IV non-small cell lung cancer patients. Many studies showed prognostic factors in stage IV non-small cell lung cancer, but no consensus on which factors are prognostic is reached and next to that, none of the prognostic factors are based on standard imaging. In this study, a previously developed radiomic signature, which was prognostic in stage I-III NSCLC and as well in head-and-neck cancer, was tested in two independent cohorts of stage IV NSCLC. In chapter 8 the association between qualitative $C T$ features and quantitative $C T$ features in stage IV NSCLC was studied. While qualitative features are inter-observer dependent, quantitative features can be scored automatically. The study investigated if a combination of radiomic features could describe semantic features, which makes it less time consuming and less inter-observer dependent. A decrease in skeletal muscle volume after 3 weeks was previously described to be prognostic for overall survival. A decrease in skeletal muscle volume probably also causes a change in the muscle texture. In chapter 9 is investigated if radiomic texture features of skeletal muscle are changed during treatment.

To conclude, chapter 10 provides a general discussion and thoughts on future perspectives on the use of image features for personalized medicine. The outline of the thesis is schematically illustrated in Figure 1.4. 


\section{References}

[1] Volksgezondheid.

URL:

https://www.volksgezondheidenzorg.info/onderwerp/kanker/.

[2] N. Karachaliou et al. "KRAS mutations in lung cancer". In: Clin Lung Cancer 14.3 (2013). Clinical lung cancer, pp. 205-14. DOI: 10.1016/j.cllc.2012.09.007.

[3] S. Novello et al. "Metastatic non-small-cell lung cancer: ESMO Clinical Practice Guidelines for diagnosis, treatment and follow-up". In: Ann Oncol 27.suppl 5 (2016). Annals of oncology : official journal of the European Society for Medical Oncology, pp. v1-v27. DOI: 10.1093/annonc/mdw326.

[4] Signaleringscommissie Kanker van K. W. F. Kankerbestrijding. Kanker in Nederland tot 2020, Trends en prognoses. Tech. rep. 2011.

[5] F. C. Detterbeck et al. "The Eighth Edition Lung Cancer Stage Classification". In: Chest 151.1 (2017). Chest, pp. 193-203. DOI: 10.1016/j.chest.2016.10.010.

[6] Cijfers over kanker. URL: https://www.cijfersoverkanker.nl/.

[7] A. M. Dingemans et al. "A randomized phase II study comparing paclitaxel-carboplatin-bevacizumab with or without nitroglycerin patches in patients with stage IV nonsquamous nonsmall-cell lung cancer: NVALT12 (NCT01171170)". In: Ann Oncol 26.11 (2015). Annals of oncology : official journal of the European Society for Medical Oncology, pp. 2286-93. DOI: $10.1093 /$ annonc/mdv370.

[8] M. G. Apps, E. H. Choi, and N. J. Wheate. "The state-of-play and future of platinum drugs". In: Endocr Relat Cancer 22.4 (2015). Endocrine-related cancer, R219-33. DOI: 10.1530 /erc-15-0237.

[9] "Drugs of choice for cancer". In: Treat Guidel Med Lett 1.7 (2003). Treatment guidelines from the Medical Letter, pp. 41-52.

[10] W. R. Wilson and M. P. Hay. "Targeting hypoxia in cancer therapy". In: Nat Rev Cancer 11.6 (2011). Nature reviews. Cancer, pp. 393-410. DOI: 10.1038/nrc3064.

[11] A. Sandler et al. "Paclitaxel-carboplatin alone or with bevacizumab for non-smallcell lung cancer". In: N Engl J Med 355.24 (2006). The New England journal of medicine, pp. 2542-50. DOI: 10.1056/NEJMoa061884.

[12] G. Mountzios. "Adjuvant bevacizumab for resected non-small cell lung cancer: the end of an era?" In: Transl Lung Cancer Res 7.Suppl 2 (2018). Translational lung cancer research, S179-s182. DOI: 10.21037/tlcr.2018.03.24.

[13] Z. Chen et al. "An essential role for mitochondrial aldehyde dehydrogenase in nitroglycerin bioactivation". In: Proc Natl Acad Sci U S A 102.34 (2005). Proceedings of the National Academy of Sciences of the United States of America, pp. 12159-64. DOI: $10.1073 /$ pnas.0503723102.

[14] D. Planchard et al. Metastatic non-small cell lung cancer: ESMO Clinical Practice Guidelines for diagnosis, treatment and follow-upt. 2018. DOI: 10.1093/annonc/ mdy275. URL: http://dx.doi.org/10.1093/annonc/mdy275.

[15] K. V. Ballman. "Biomarker: Predictive or Prognostic?" In: J Clin Oncol 33.33 (2015). Journal of clinical oncology : official journal of the American Society of Clinical Oncology, pp. 3968-71. DOI: 10.1200/jco.2015.63.3651. 
[16] F. R. Vogenberg. "Predictive and prognostic models: implications for healthcare decision-making in a modern recession". In: Am Health Drug Benefits 2.6 (2009). American health \& drug benefits, pp. 218-22.

[17] J. Sorensen. "Lung Cancer Cachexia: Can Molecular Understanding Guide Clinical Management?" In: Integr Cancer Ther (2018). Integrative cancer therapies, p. 1534735418781743 . DOI: $10.1177 / 1534735418781743$.

[18] G. E. Darling et al. "Positron emission tomography-computed tomography compared with invasive mediastinal staging in non-small cell lung cancer: results of mediastinal staging in the early lung positron emission tomography trial". In: J Thorac Oncol 6.8 (2011). Journal of thoracic oncology : official publication of the International Association for the Study of Lung Cancer, pp. 1367-72. DOI: 10.1097/JTO.0b013e318220c912.

[19] P. M. Smith-Jones. "Drug Discovery Technologies". In: Comprehensive Medicinal Chemistry II. 2007.

[20] E. E. Verwer, R. Boellaard, and A. A. van der Veldt. "Positron emission tomography to assess hypoxia and perfusion in lung cancer". In: World J Clin Oncol 5.5 (2014). World journal of clinical oncology, pp. 824-44. DOI: 10.5306/wjco.v5.i5.824. URL: http://www.ncbi.nlm.nih.gov/pubmed/25493221.

[21] National Institute of Biomedical Imaging Bioengineering. Magnetic Resonance Imaging. URL: https:// www . nibib . nih . gov / science - education / science topics/magnetic-resonance-imaging-mri.

[22] R. Boellaard et al. "FDG PET and PET/CT: EANM procedure guidelines for tumour PET imaging: version 1.0". In: Eur J Nucl Med Mol Imaging 37.1 (2010). European journal of nuclear medicine and molecular imaging, pp. 181-200. DOI: 10.1007/ s00259-009-1297-4.

[23] R. Boellaard et al. "FDG PET/CT: EANM procedure guidelines for tumour imaging: version 2.0". In: Eur J Nucl Med Mol Imaging 42.2 (2015). European journal of nuclear medicine and molecular imaging, pp. 328-54. DOI: $10.1007 /$ s00259-0142961-x. URL: http://www.ncbi.nlm.nih.gov/pubmed/25452219.

[24] R. Boellaard et al. "The Netherlands protocol for standardisation and quantification of FDG whole body PET studies in multi-centre trials". In: Eur J Nucl Med Mol Imaging 35.12 (2008). European journal of nuclear medicine and molecular imaging, pp. 2320-33. DOI: 10.1007/s00259-008-0874-2. URL: http://www.ncbi.nlm.nih. gov/pubmed/18704407.

[25] CT protocol screening AAPM. URL: https://www.aapm.org/pubs/CTProtocols/ documents/LungCancerScreeningCT.pdf.

[26] AAPM. Routine Adult Chest CT protocols. 2016. URL: https://www.aapm.org/ pubs/CTProtocols/documents/AdultRoutineChestCT.pdf.

[27] J. P. O'Connor et al. "Imaging biomarker roadmap for cancer studies". In: Nat Rev Clin Oncol 14.3 (2017). Nature reviews. Clinical oncology, pp. 169-186. DOI: 10.1038 /nrclinonc. 2016.162 .

[28] S. Rizzo et al. "CT Radiogenomic Characterization of EGFR, K-RAS, and ALK Mutations in Non-Small Cell Lung Cancer". In: Eur Radiol 26.1 (2016). European radiology, pp. 32-42. DOI: 10.1007/s00330-015-3814-0. 
[29] S. S. F. Yip et al. "Associations between radiologist-defined semantic and automatically computed radiomic features in non-small cell lung cancer". In: Sci Rep 7.1 (2017). Scientific reports, p. 3519. DOI: 10.1038/s41598-017-02425-5.

[30] P. Lambin et al. "Radiomics: extracting more information from medical images using advanced feature analysis". In: Eur J Cancer 48.4 (2012). European journal of cancer (Oxford, England : 1990), pp. 441-6. DOI: 10.1016/j.ejca.2011.11.036.

[31] H. J. Aerts et al. "Decoding tumour phenotype by noninvasive imaging using a quantitative radiomics approach". In: Nat Commun 5 (2014). Nature communications, p. 4006. DOI: $10.1038 /$ ncomms5006. 



\section{Chapter 2}

\section{Radiomics applied to lung cancer: a review}

\section{Authors}

Madeleine Scrivener*, Evelyn E.C. de Jong*, Janna E. van Timmeren*, Thierry Pieters, Benôit Ghaye, Xavier Geets

\section{Adapted from}

Translational Cancer Research, 2016 August; 5(4): 389-409 ISSN: 2219-6803

*equal contribution 


\section{Abstract}

Lung cancers exhibit strong phenotypic differences that can be visualized noninvasively by medical imaging. Radiomics, a concept introduced in 2012, refers to the comprehensive quantification of tumor phenotypes by applying a large number of quantitative image features (watch the animation: https://youtu.be/Tq980GEVP0Y and the website www.radiomics.org). Here, we review the literature related to radiomics for lung cancer. We found 11 papers related to computed tomography (CT) radiomics, 3 to radiomics or texture analysis with positron emission tomography (PET) and 8 relating to $\mathrm{PET} / \mathrm{CT}$ radiomics. There are two main applications of radiomics, the classification of lung nodules (diagnostic) or prognostication of established lung cancer (theragnostic). There are quite a few methodological issues in most of the reviewed papers. Only 5 studies, out of the 22, were externally validated. Overall, it is clear that radiomics offers great potential in improving diagnosis and patient stratification in lung cancer. It may also have a real clinical impact, as imaging is routinely used in clinical practice, providing an unprecedented opportunity to improve decision support in lung cancer treatment at low cost. 


\section{Introduction}

Globally, lung cancer is the most frequent cause of death in men and the second most frequent cause amongst women in the USA [1]. Past smoking patterns strongly influence actual cancer rates amongst both men and women and vary considerably due to geographical location, age, race, and socioeconomic status. There is a broad consensus that it is necessary to develop better biomarkers predicting treatment response or survival, in order to identify subgroups that would benefit from individualized treatment to improve the patient's outcome.

Most tumours do not represent a homogenous entity; they are composed of multiple clonal sub-populations of cancer cells. This heterogeneous complex needs to be analysed in order to tailor the cancer care to the patient and their tumour. One way of characterizing the tumour is by extracting tumour tissue in a tumour biopsy, the tissue is then characterised using genomics based approaches. Although these approaches have successfully been used in clinical oncology, there are intrinsic limitations; repeated tumour biopsies increase the risk of complications for the patients, in particular in lung cancer and the results may vary depending on which part of the tumour is biopsied.

These challenges can be addressed by medical imaging. Medical imaging is an important part of cancer care, it is essential to cancer staging and diagnosis. Unlike biopsies, it is noninvasive, three dimensional and provides information regarding the entire tumour.

Radiomics, first introduced by Lambin et al. [2] in 2012 and summarized in a two minute animation (http://youtu.be/Tq980GEVP0Y), is a process that converts standard of care images into minable high-dimensional data. The radiomics process can extract quantitative features from digital medical images, which can then be used to build descriptive and predictive models, linking the image features to the tumour's gene-protein signatures or the tumour's phenotype. As shown in recent studies, quantitative imaging features have a prognostic value and potential in predicting clinical outcomes or treatment monitoring in different cancer types $[3,4,5,6,7]$. The aim of radiomics is to use these models, which can include biological or medical data, to help provide valuable diagnostic, prognostic or predictive information. Radiomics aims to utilise the full potential of medical imaging by extracting and analysing large amounts of advanced quantitative imaging features, with high throughput from digital medical images, obtained with computed tomography (CT), positron emission tomography (PET) or magnetic resonance imaging $(\mathrm{MRI})$. This in turn provides a more detailed quantification of tumour phenotypic characteristics describing its intensity, shape, texture, intra-tumour heterogeneity and in doing so it effectively converts medical images into a high dimensional minable feature space.

Precision oncology is the customization of cancer care where therapies and/or practices are being tailored to individual patients, with all of the information about the tumour characteristics that radiomics provides; it presents numerous new opportunities for precision medicine.

In this paper we will review the literature and discuss the potential advantages and pitfalls of radiomics in lung cancer. 


\section{Workflow of radiomics}

The radiomics process consists of three steps: (I) image acquisition and volume segmentation; (II) feature extraction and storage; (III) signature development and validation on one or several datasets (see Figure 2.1) [2, 8, 9]. Each of these three steps poses unique challenges, which will be introduced below. Once a signature has been developed and is applied on a specific patient, the process is the same except that in step 3 the validated signature is used to determine the prognosis of the patient.

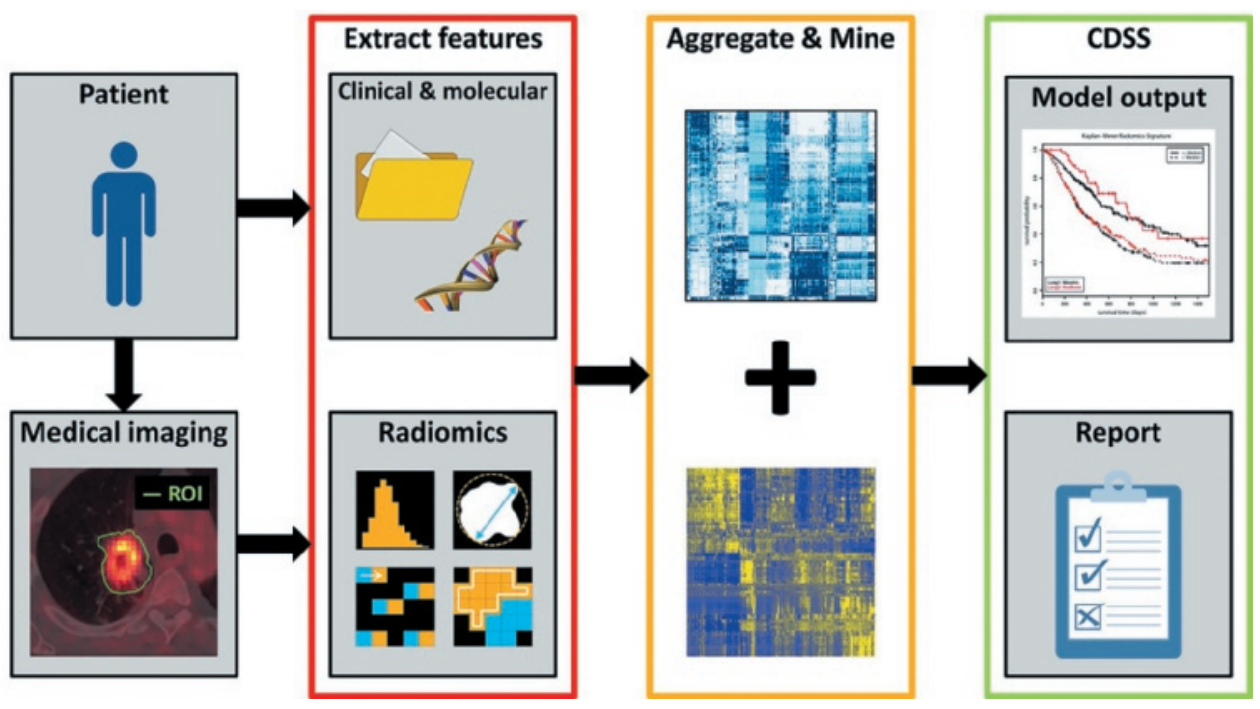

Figure 2.1: Overview of methodological processes in radiomics: data discovery, collection and preparation, model(s) development/validation and implementation, assessment of clinical utility.

\section{Image acquisition}

Modern CT, PET and MRI scanners allow acquisition and reconstruction settings in a large range. Although this facilitates the subjective needs of the human expert, when the images are intended to be objectively characterized by a machine, these variations may create a bias that masks the true underlying biological characteristics. This phenomenon is well recognized in the field of radiomics and consequently, efforts are being made to standardize acquisition and reconstruction protocols. This advance in quantitative imaging is being led by several organizations or consortia such as the European Association of Nuclear Medicine (EANM), the QulC-ConCePT project from the Innovative Medicine Initiative Joint Undertaking (IMIJU).

\section{Volumes of interest}

One of the central processes of radiomics is to identify one or several volumes of interest (the primary tumour, nodules, etc.) on the diagnostic imaging. However, predictive value may lie in the detailed analysis of subvolumes within the tumour, also known as habitats. The heterogeneity within the tumour is due to particular combinations of blood 
flow, oedema, necrosis, and cell density, which creates a unique pathophysiology. Using radiomics, important additional information from these habitats as well as information from a normal healthy lung, can be extracted.

\section{Segmentation}

Segmentation or delineation plays a crucial role within radiomics, because the features that are generated depend on the segmented volumes. However, many tumours, as well as subvolumes, have ambiguous borders. This can lead to a significant inter-reader bias and low reproducibility when these volumes are manually delineated. Unfortunately, there is no universal automatic segmentation algorithm that can work for all medical images $[10,11]$. Consequently, the consensus that emerges out of this debate is that the optimum reproducible segmentation can be obtained via semi-automatic segmentation, which consists of automatic segmentation followed by, if necessary, manual curation [12]. The different image modalities have also their own segmentation methods. For CT, the region of interest (ROI) represents for example the gross tumor volume (GTV), while for PET the metabolic target volume (MTV) is segmented as ROI. The segmentation method also depends on the endpoint of the study. Cheebsumon et al. [13] showed that CT-based delineation was overestimated compared to pathology while PET-based tumour delineation methods provided maximum diameters in closer agreement with pathology. They also showed that for example the contrast-oriented methods seem to best suited for assessing tumour size, while an adaptive $70 \%$ threshold-based method is better for response monitoring [13].

\section{Feature extraction}

The essential part of radiomics is the high throughput extraction of quantitative image features that characterize the volumes of interest. The number of features is enormous, more then 1000, and complex, and this leads to the risk of overfitting. Overfitting exists when a model is specifically and exclusively optimized for the training dataset and consequently performs poorly on new data. To avoid overfitting, the ratio of the number of evaluated features to the number of outcome events must be kept as low as possible. To offset this risk, there is a key process of feature reduction and ranking. In order to do so, one approach consists in looking at robustness and reproducibility of features in test-retest datasets. The information extracted from two datasets of images acquired within a small period of time (few minutes to few days) from a single patient cohort is called test-retest data and is highly advantageous for ranking features thanks to their reproducibility and stability. Therefore, when coupled with robust segmentation, test-retest data should be exploited whenever possible. Another complementary approach consists of identifying features that may be redundant, if they are for example, highly correlated with one another. Groups of highly correlated features can in turn be reduced to one archetypal feature.

\section{Model development and validation}

Developing a model based on the calculated radiomics features can be data-driven or hypothesis driven. The data-driven approach makes no assumption about the meaning of individual features, therefore all features are treated with equal weight during model development, whereas the hypothesis-driven approach treats cluster features according 
to predefined information content and a clinical context. The best models start with a well-defined end-point, such as overall survival (OS), and ideally can accommodate non-radiomics features. Covariates that need to be taken into account include clinical, treatment and genomic data (age, histology, tumour-node-metastasis (TNM) stage, serum markers, chemotherapy, radiotherapy, dose, fractionation, treatment time, gene expression, mutation status, gene polymorphisms...). This information is occasionally missing for some patients; therefore models should accommodate sparse data. It is necessary that the models be adequately validated with an external dataset, preferably from an external institute. If data from an external institute is not available, the available data must be split into a model training dataset and a validation dataset.

Model performance is often measured in terms of calibration and discrimination. Accurate models correctly discriminate between patients. This can be measured using the c-index or the area under the curve (AUC) of receiver operating characteristic (ROC) for censored data [14]. On the other hand, the calibration is the association between observed outcomes and model prediction.

\section{Analysis of the literature}

Reviewing all the literature about radiomics in lung cancer has shown that radiomics can be useful in many fields, such as in the classification of nodules, in the description of the tumour, and it has already been shown to be a tool that can assess patient prognosis [3, 15]. All of the articles published to date, to the best of our knowledge, about radiomics in lung cancer have been placed in Table 2.1.

First of all, 16 articles in the table assess the prognostic value of texture analysis from standard of care CT and FDG-PET images, which can predict treatment outcome in some ways.

Certain methodological aspects of radiomics have been studied, for example, in order to find a way of reducing redundancy and comparing the prognostic characteristics of radiomics features across cancer types. Parmar et al. [16], investigated cancer-specific radiomics feature clusters in his study published in 2015 . This study concluded that consensus clustering could provide robust radiomics feature clusters and therefore could reduce feature redundancy. Another study, published by Leijenaar et al. [17] analysed the test-retest and inter-observer variability of radiomics features in FDG-PET images. The study concluded that the majority of assessed features had both a high test-retest $(71 \%)$ and inter-observer (91\%) stability, and that overall, features that were more stable in repeated PET imaging were also found to be more robust against inter-observer variability.

One of the first studies showing the potential of CT texture analysis as independent marker of survival for patients with non-small cell lung cancer (NSCLC) is the study of Ganeshan et al. [18] from 2012. They showed that patients with heterogeneous tumours with low uniformity values demonstrated poorer survival and that CT texture and PET stage were significant independent predictors of survival. Another study of Balagurunathan et al. [8] showed that a large value of run length calculated on CT images (one of the reproducible features) indicated a more homogeneous tumour, and that this was related to a longer survival. The largest, the most comprehensive and rigorous study is from Aerts et al. [15] who worked on radiomics to decode the tumour phenotype, found that a large number of radiomics features have prognostic power in independent datasets of lung and head and neck cancer patients, many of which were previously not identified as 
significant. After a very strict features reduction process, using test-retest datasets and multiple delineations datasets, they created a signature consisting of "only" four features quantifying tumour heterogeneity, consistently with their initial hypothesis. Combining this radiomics signature with TNM staging showed a significant improvement in all data sets, compared with TNM staging alone, considered as gold standard. Remarkably, the same signature was working on lung and head neck cancer suggesting that radiomics identifies a general prognostic phenotype existing in both lung and head and neck cancer. They also showed in a series of operated patients, having had standardized CT imaging and gene expression array, the so-called "radiogenomic approach", that the four feature prognostic radiomics signature, capturing intratumour heterogeneity, is associated with underlying gene-expression patterns linked to tumour proliferation [19]. Overall, this study convincingly validate the signature on three external datasets and recently in a fourth one [20].

Next to the studies describing radiomics alone to stratify patients, there are also some studies describing the value of adding radiomics features to conventional prognostic factors alone. A study of Desseroit et al. [21] described the development of a nomogram combining clinical staging with PET/CT image features stage I-III NSCLC patients. The nomogram had a higher stratification power than the clinical staging alone. Patients with stage III disease, with a large tumour volume, low CT heterogeneity although a high PET heterogeneity had the poorest prognosis. A study of Fried et al. [4] also showed that models using textural features and conventional prognostic factors improved stratification power compared to models using conventional prognostic factors alone.

In addition, it was found that radiomics features can reflect different biologic mechanisms, such as gene-expression patterns or cell cycling pathways. A study of Yoon et al. [22] also made the link with gene-expression and showed that quantitative imaging using radiomics can capture distinct phenotypic differences between tumours. They showed that ALK/ROS1/RET fusion positive lung adenocarcinomas possesses certain imaging features next to clinical features that enable good discrimination between fusion positive and fusion negative tumours.

Three articles studied the imaging texture analysis of lung cancer in patients treated by stereotactic radiotherapy $[23,24,25]$. Two studies by Mattonen et al. $[24,25]$ investigated the use of imaging texture analysis in a decision support system in order to support earlier salvage for patients with recurrence and fewer investigations of benign radiation-induced lung injury. They found that radiomic features, extracted from CT images using an automated method, are able to predict recurrence with better performance than physicians. The study of Lovinfosse et al. [23] used radiomics along with clinical features, using univariate and multivariate analysis for OS, disease-specific survival (DSS) and disease-free survival (DFS) in order to assess the predictive value of the radiomics features. They concluded that the textural feature "dissimilarity" measured on the baseline ${ }^{18} \mathrm{~F}$-FDG PET/CT appears to be a strong independent predictor of the outcome in patients with NSCLC treated by stereotactic body radiation therapy.

Prognostic value of metabolic metrics extracted from baseline PET images in NSCLC is studied in three separate articles using OS to assess the prognostic value $[5,7,26]$. The study from 2013 by Carvalho et al. [5] examined the prognostic value of metabolic PET descriptors on OS for NSCLC patients and they showed that only one metric, relative volume above $80 \%$ SUV, was significantly related to OS $(p=0.05)$.

The study from Cook et al. [7] investigated the relation of radiomics features to response and survival after chemoradiotherapy for 53 NSCLC patients. This study 


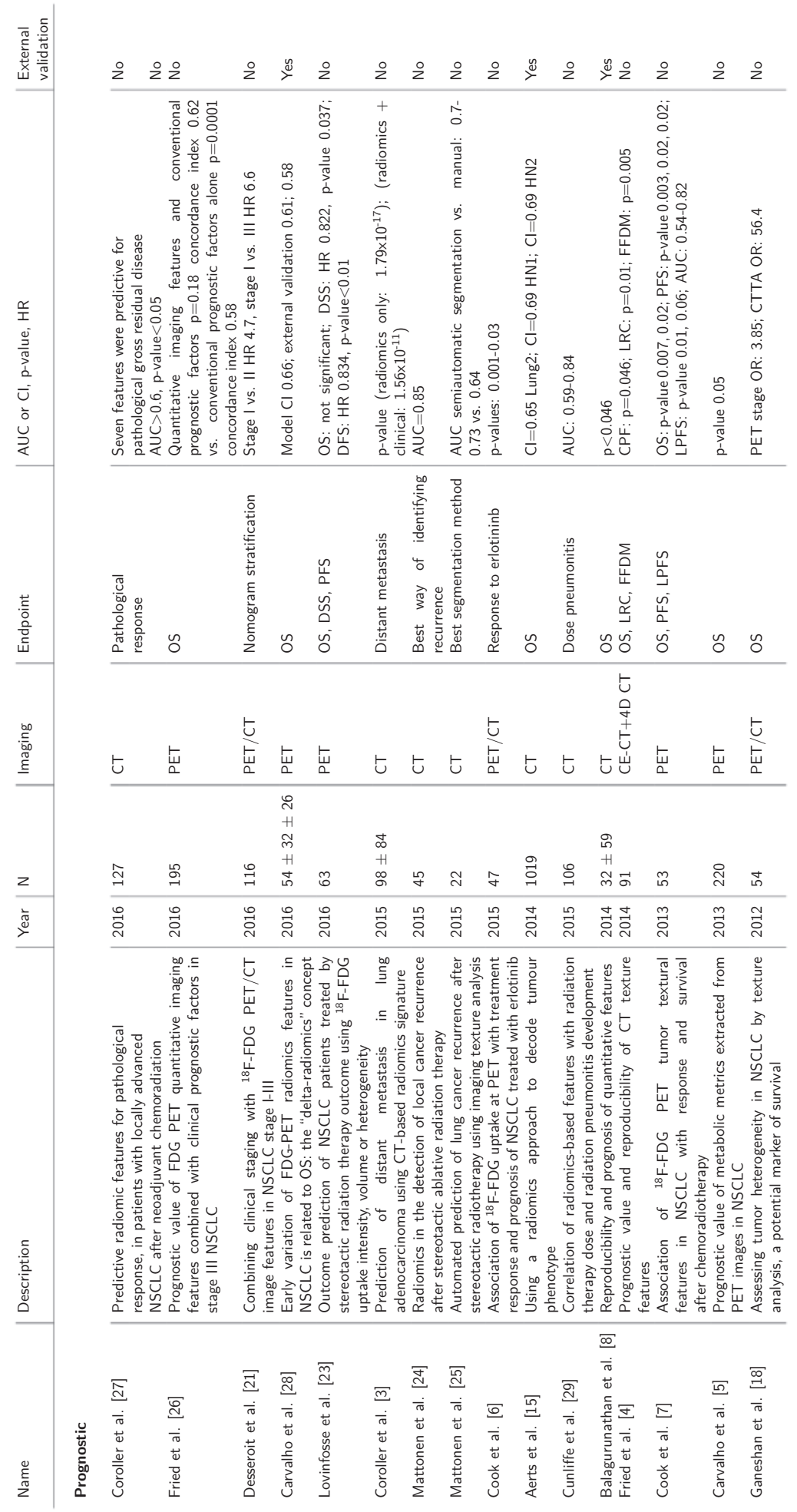




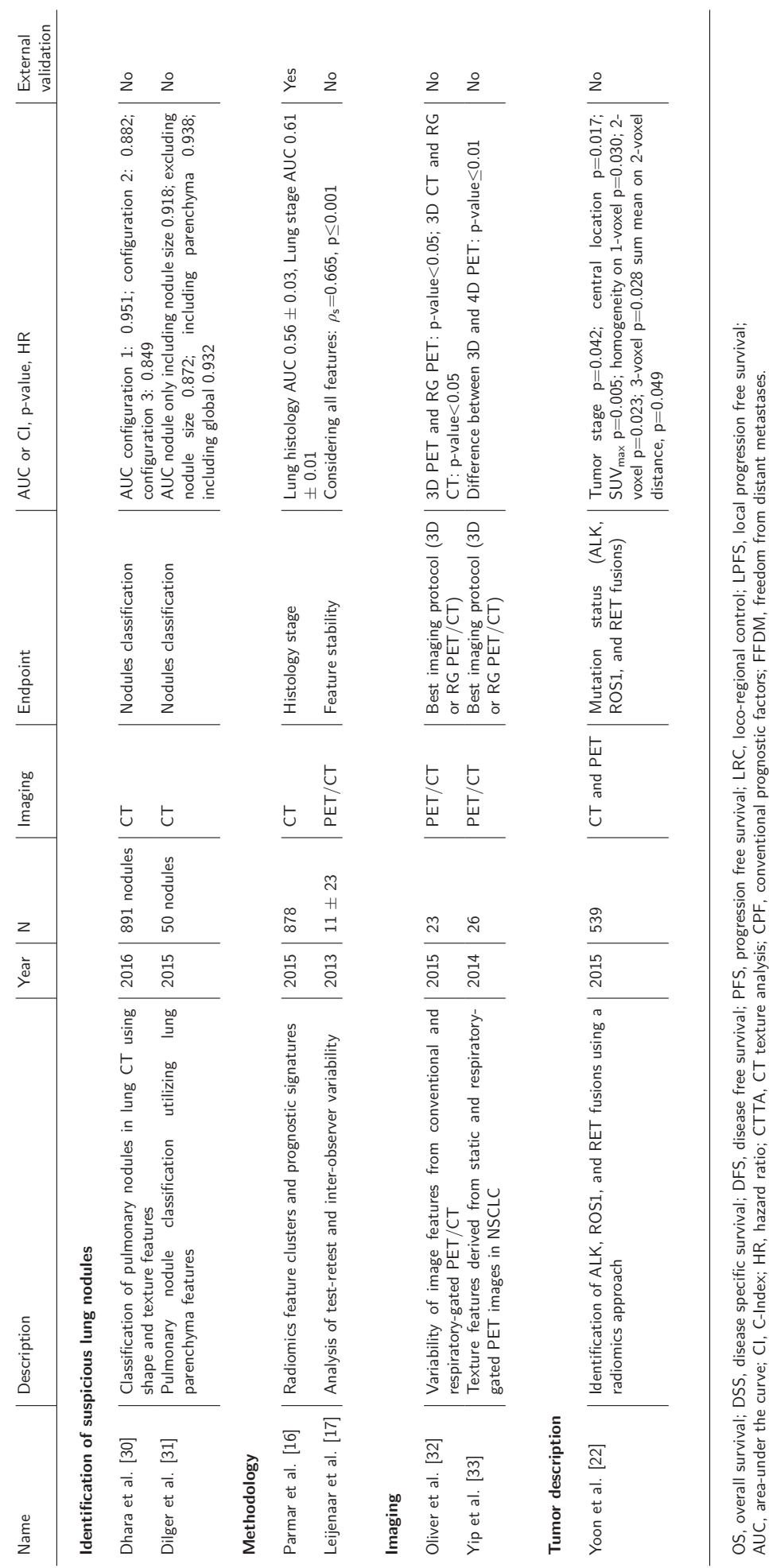


concluded that three textural features were able to differentiate between responders and non-responders to chemoradiotherapy (determined using RECIST) and were independent predictors of OS.

A study by Cook et al. [6] showed that the response to erlotinib is associated with reduced heterogeneity at ${ }^{18} \mathrm{~F}-\mathrm{FDG}$ PET and that changes in first-order entropy are independently associated with OS and treatment response.

And the 2016 study by Fried et al. [26] showed that pretreatment PET features were associated with OS in 195 patients with stage III NSCLC. In this study, predictors of OS generated with both quantitative imaging features and conventional prognostic factors demonstrated improved risk stratification compared with those generated with conventional prognostic factors.

Another study investigating the prognostic value of PET features by van Gómez López et al. [34] showed that textural analyses of ${ }^{18}$ F-FDG PET images to assess tumour heterogeneity were related to global metabolic parameters (e.g., SUV max $, S_{\text {mean }}, M T V$ and TLG) and pathologic staging.

In addition, the study by Coroller et al. [27] identified predictive radiomic features for pathological response in 127 patients with locally advanced NSCLC after neoadjuvant chemoradiation. This study demonstrated that radiomics can provide valuable clinical information, and perform better than conventional imaging.

Furthermore, one study published in 2015 used radiomics to predict tumour distant metastasis, and concluded that radiomics features capture detailed information of the tumour phenotype and therefore can be used as a prognostic biomarker for clinicallyrelevant factors such as distant metastasis [3]. However, distant metastasis is not the only clinically-relevant factor with which radiomics features can be correlated. A different study assessed the relationship between radiation dose and change of texture-based features of lung tissue in order to determine the ability of texture analysis to identify patients who develop radiation pneumonitis (RP). A relationship between dose and change in a set of image-based features was observed. For 12 features, feature values were significantly related to RP development. This study demonstrated the ability of radiomics to provide a quantitative, individualized measurement of patient lung tissue reaction to radiation therapy and assess RP development.

In the field of diagnostics, radiomics can be used for the classification of nodules. A study by Dhara et al. [30] showed that using support vector machine nodules can be classified as benign or malignant. In addition the study by Dilger et al. [31] showed that this classification can be improved by including features quantified from the surrounding lung tissue.

Radiomics is usually performed using textural information derived from the primary tumor. However, textural information derived from the lymph nodes may contain complementary information. An ongoing study on ${ }^{18}$ F-FDG PET images of 262 NSCLC patients is performing radiomics on both primary tumour and lymph nodes and is investigating the predictive performance to OS [35].

The above-mentioned studies performed radiomics on pre-treatment scans to advance the stratification of patients for therapy leading to improvements in health outcomes. Some other studies focused more on radiomics for response assessment by comparing radiomics features of a baseline scan with radiomics features of a second scan performed during treatment. Information about early changes in radiomics features can potentially increase the prognostic value of a model. Currently, percentage variations of radiomics features derived from PET images at baseline and the second week of treatment are calculated 
and related to OS for NSCLC patients [28]. The next challenge will be to use radiomics approaches for PET biomarkers different then fluorodeoxyglucose in an attempt to extract more information [36, 37, 38, 39].

\section{Challenges and future prospects}

\section{Tumour motion}

Standardization of image acquisition and reconstruction is one of the major challenges of radiomics [40, 41, 42]. Reproducibility and stability of radiomics features is important for potential clinical utility $[12,17,43]$. In lung cancer, breathing-induced tumour motion is one of the factors causing variability in image features, and different methods exist to reduce the influence of breathing motion on image characteristics [44]. Due to the scan duration of a 3D (free-breathing) PET scan, images are averaged over multiple breathing cycles and introduce some noise. During a respiratory correlated 4D PET acquisition, counts are binned into typically 5 to 10 phases. This gating based on respiratory motion reduces blurring, but due to the reduced acquisition time per phase an increased noise is observed. However, there are also motion correction techniques available $[45,46,47]$. For CT images of lung cancer patients, the mid-ventilation phase of a respiratory-gated (4D) acquisition is commonly used, in the motion management option, for target delineation. This phase is typically used for treatment planning purposes and the GTV or MTV can also be used as the ROI for tumour quantification with radiomics. Variations in radiomics feature values may occur when a different phase or a static (3D) CT is used instead. In Figure 2.2, variations in $\mathrm{SUV}_{\max }$ are shown using different acquisition methods.

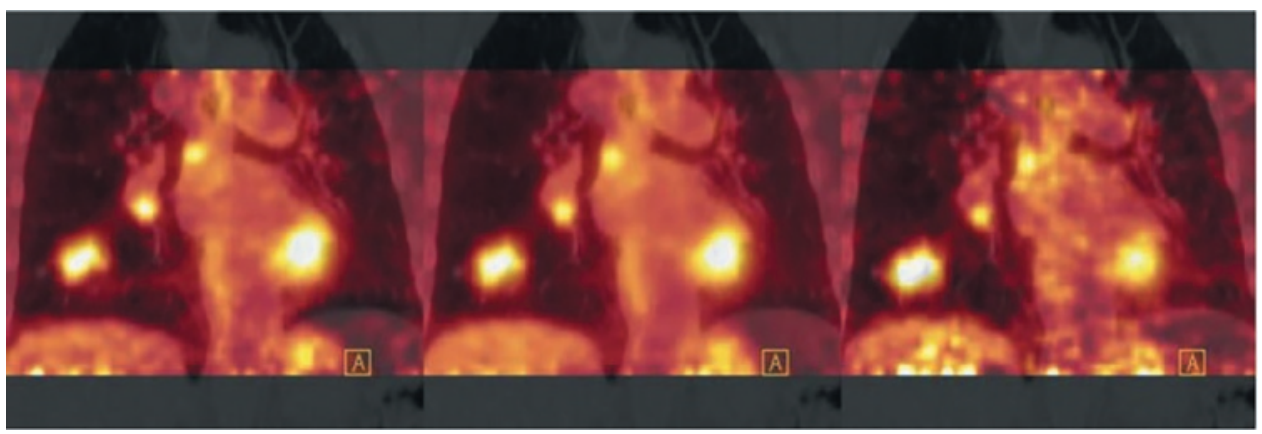

Figure 2.2: Fused PET/CT images obtained with optimal gating (left), 3D reconstruction (middle) and 3D reconstruction (4D), showing variations in $\mathrm{SUV}_{\max }$ [45]. PET, positron emission tomography; CT, computed tomography.

For generalizability and robust extraction of radiomics for lung cancer, it is important that the impact of tumour motion on the radiomics feature values is minimized, using gated acquisitions or breath-hold methods.

Two recent studies investigated the influence of motion on radiomics feature values derived from PET and CT. Relative differences in features values were calculated between $3 \mathrm{D}$ and $4 \mathrm{D}$ acquisitions. A study by Yip et al. [33] investigated five texture features derived from 3D PET and 4D PET for 26 lung cancer patients. In this study, 4D PET counts were binned into five phases and the differences in features values between all five 
phase bins were assessed. They reported significant differences between 3D and all bins of 4D PET for three features and significant differences between 3D and 4D for four out of five bins for one feature. The textural feature 'Contrast' was not significantly different between 3D and 4D PET. Between different breathing phases, none of the five features was significantly different. A second study evaluated the influence of 56 radiomics features between 3D PET/CT and 4D PET/CT acquisitions for lung cancer patients $(n=23)$ [32]. The acquired data from the PET scan were binned into ten phases. Features were extracted from the first phase (inhale) and the fifth phase (exhale) of the respiratory-gated scan. They found that the differences in features between a 3D and 4D acquisitions varied between $0 \%$ and $193 \%$ for PET and $0 \%$ and $176 \%$ for CT, with the feature kurtosis being an outlier for both modalities. Substantial differences between radiomics features derived from 3D and 4D acquisitions were observed for both CT and PET. Respiratorygated imaging reduces effects of motion, including blurring, rotation and deformation at the expense of a somewhat higher noise level. It is important to choose the respiratory phase-bin with robust features for quantitative tumour characterization using 4D imaging. To improve standardization in radiomics, acquisition and reconstruction protocols used to acquire quantitative image features should always be described in detail for both PET and CT.

\section{Methodological issues}

There is one major risk in radiomics studies: the selection of significant features by chance and overfitting. This issue is very similar to that in the field of genomics. Authors should clearly state in their publication, in what way the study has advanced the field of radiomics and how it has specifically identified and met an unmet need. It is also important that authors avoid making overly optimistic claims concerning robustness and generalizability as they diminish scientific and clinical impact. Furthermore, only 5 of the 22 articles were externally validated, this highlights another methodological issue. Study design, protocols, detailed quality assurance processes and standard operating procedures should be exhaustively reported in the publications. Rigorous reporting guidelines and requirements are necessary for the maturation of radiomics. While the minute technical details of radiomics and finding external validation datasets can be tedious, they potentially have a great influence on the robustness, generalizability and confound meta-analyses. The following points are crucial in radiomics studies:

1. I. Standardized imaging protocols allow for appropriate meta-analysis;

2. II. The effects of inter-scanner differences should considered and minimized;

3. III. Robust segmentation, preferably automated, is advantageous for ranking features on the basis of their spatial reproducibility/stability;

4. IV. Test-retest is useful for ranking features on the basis of their temporal reproducibility/stability [17];

5. V. Independent validation datasets, preferably from another centre and multiple datasets can provide crucial information with regard to overfitting, clinical performance and generalizability. 


\section{Conclusions}

There are two main applications of radiomics in lung cancer, classification of lung nodules (diagnostic) or prognostication of established lung cancer (theragnostic). Overall, it is clear that radiomics has great potential to improve diagnosis and patients stratification in lung cancer. It may also have a clinical impact as imaging is routinely used in clinical practice, providing an unprecedented opportunity to improve decision support in lung cancer for diagnosis or treatment at low cost $[48,49,50,51]$.

\section{Acknowledgements}

Authors acknowledge financial support from the QulC-ConCePT project, which is partly funded by EFPI Acompanies and the Innovative Medicine Initiative Joint Undertaking (IMIJU) under Grant Agreement No. 115151. This research is also supported by the Dutch technology Foundation STW (grant n 10696 DuCAT; n P14-19 radiomics STRaTegy), which is the applied science division of NWO. Authors also acknowledge financial support from EU 7th framework program (ARTFORCE - n 257144, REQUITE - n 601826), SME Phase 2 (EU proposal 673780 - RAIL). Authors thank the contribution of Simone Moorman for editing. 


\section{References}

[1] Cancer statistics. URL: https://cancerstatisticscenter.cancer.org.

[2] P. Lambin et al. "Radiomics: extracting more information from medical images using advanced feature analysis". In: Eur J Cancer 48.4 (2012). European journal of cancer (Oxford, England : 1990), pp. 441-6. DOI: 10.1016/j.ejca.2011.11.036.

[3] T. P. Coroller et al. "CT-based radiomic signature predicts distant metastasis in lung adenocarcinoma". In: Radiother Oncol 114.3 (2015). Radiotherapy and oncology : journal of the European Society for Therapeutic Radiology and Oncology, pp. 345-50. DOI: 10.1016/j.radonc.2015.02.015.

[4] D. V. Fried et al. "Prognostic value and reproducibility of pretreatment CT texture features in stage III non-small cell lung cancer". In: Int J Radiat Oncol Biol Phys 90.4 (2014). International journal of radiation oncology, biology, physics, pp. 834-42. DOI: $10.1016 /$ j.ijrobp.2014.07.020.

[5] S. Carvalho et al. "Prognostic value of metabolic metrics extracted from baseline positron emission tomography images in non-small cell lung cancer". In: Acta Oncol 52.7 (2013). Acta oncologica (Stockholm, Sweden), pp. 1398-404. DOI: 10.3109/ 0284186x.2013.812795.

[6] G. J. Cook et al. "Non-Small Cell Lung Cancer Treated with Erlotinib: Heterogeneity of (18)F-FDG Uptake at PET-Association with Treatment Response and Prognosis". In: Radiology 276.3 (2015). Radiology, pp. 883-93. DOI: 10.1148/radiol.2015141309.

[7] G. J. Cook et al. "Are pretreatment 18F-FDG PET tumor textural features in non-small cell lung cancer associated with response and survival after chemoradiotherapy?" In: J Nucl Med 54.1 (2013). Journal of nuclear medicine : official publication, Society of Nuclear Medicine, pp. 19-26. DOI: 10.2967/jnumed.112.107375.

[8] Y. Balagurunathan et al. "Reproducibility and Prognosis of Quantitative Features Extracted from CT Images". In: Transl Oncol 7.1 (2014). Translational oncology, pp. $72-87$.

[9] V. Kumar et al. "Radiomics: the process and the challenges". In: Magn Reson Imaging 30.9 (2012). Magnetic resonance imaging, pp. 1234-48. DOI: 10.1016/j.mri.2012.06.010.

[10] E. R. Velazquez et al. "Volumetric CT-based segmentation of NSCLC using 3DSlicer". In: Sci Rep 3 (2013). Scientific reports, p. 3529. DOI: 10.1038/srep03529.

[11] M. Hatt et al. "Impact of tumor size and tracer uptake heterogeneity in (18)FFDG PET and CT non-small cell lung cancer tumor delineation". In: J Nucl Med 52.11 (2011). Journal of nuclear medicine : official publication, Society of Nuclear Medicine, pp. 1690-7. DOI: 10.2967/jnumed.111.092767.

[12] C. Parmar et al. "Robust Radiomics feature quantification using semiautomatic volumetric segmentation." eng. In: PloS one 9 (7 2014), e102107.

[13] P. Cheebsumon et al. "Assessment of tumour size in PET/CT lung cancer studies: PET- and CT-based methods compared to pathology". In: EJNMMI Res 2.1 (2012). EJNMMI research, p. 56. DOI: 10.1186/2191-219x-2-56. 
[14] E. W. Steyerberg et al. "Assessing the performance of prediction models: a framework for traditional and novel measures". In: Epidemiology 21.1 (2010). Epidemiology (Cambridge, Mass.), pp. 128-38. DOI: 10.1097/EDE.0b013e3181c30fb2.

[15] H. J. Aerts et al. "Decoding tumour phenotype by noninvasive imaging using a quantitative radiomics approach". In: Nat Commun 5 (2014). Nature communications, p. 4006. DOI: $10.1038 /$ ncomms5006.

[16] C. Parmar et al. "Radiomic feature clusters and prognostic signatures specific for Lung and Head \& Neck cancer". In: Sci Rep 5 (2015). Scientific reports, p. 11044. DOI: $10.1038 /$ srep11044.

[17] R. T. Leijenaar et al. "Stability of FDG-PET Radiomics features: an integrated analysis of test-retest and inter-observer variability". In: Acta Oncol 52.7 (2013). Acta oncologica (Stockholm, Sweden), pp. 1391-7. DOI: 10.3109/0284186x.2013. 812798.

[18] B. Ganeshan et al. "Tumour heterogeneity in non-small cell lung carcinoma assessed by CT texture analysis: a potential marker of survival". In: Eur Radiol 22.4 (2012). European radiology, pp. 796-802. DOI: 10.1007/s00330-011-2319-8.

[19] K. M. Panth et al. "Is there a causal relationship between genetic changes and radiomics-based image features? An in vivo preclinical experiment with doxycycline inducible GADD34 tumor cells". In: Radiother Oncol 116.3 (2015). Radiotherapy and oncology : journal of the European Society for Therapeutic Radiology and Oncology, pp. 462-6. DOI: 10.1016/j.radonc.2015.06.013.

[20] R. T. Leijenaar et al. "External validation of a prognostic CT-based radiomic signature in oropharyngeal squamous cell carcinoma". In: Acta Oncol 54.9 (2015). Acta oncologica (Stockholm, Sweden), pp. 1423-9. DOI: 10.3109/0284186x.2015.1061214.

[21] M. C. Desseroit et al. "Development of a nomogram combining clinical staging with (18)F-FDG PET/CT image features in non-small-cell lung cancer stage I-III". In: Eur J Nucl Med Mol Imaging 43.8 (2016). European journal of nuclear medicine and molecular imaging, pp. 1477-85. DOI: 10.1007/s00259-016-3325-5.

[22] H. J. Yoon et al. "Decoding Tumor Phenotypes for ALK, ROS1, and RET Fusions in Lung Adenocarcinoma Using a Radiomics Approach". In: Medicine (Baltimore) 94.41 (2015). Medicine, e1753. DOI: 10.1097/md.0000000000001753.

[23] P. Lovinfosse et al. "FDG PET/CT texture analysis for predicting the outcome of lung cancer treated by stereotactic body radiation therapy". In: Eur J Nucl Med Mol Imaging 43.8 (2016). European journal of nuclear medicine and molecular imaging, pp. 1453-60. DOI: 10.1007/s00259-016-3314-8.

[24] S. A. Mattonen et al. "Detection of Local Cancer Recurrence After Stereotactic Ablative Radiation Therapy for Lung Cancer: Physician Performance Versus Radiomic Assessment". In: Int J Radiat Oncol Biol Phys 94.5 (2016). International journal of radiation oncology, biology, physics, pp. 1121-8. DOI: 10.1016/j.ijrobp.2015.12.369.

[25] S. A. Mattonen et al. "Imaging texture analysis for automated prediction of lung cancer recurrence after stereotactic radiotherapy". In: J Med Imaging (Bellingham) 2.4 (2015). Journal of medical imaging (Bellingham, Wash.), p. 041010. DOI: 10. 1117/1.jmi.2.4.041010. 
[26] D. V. Fried et al. "Stage III Non-Small Cell Lung Cancer: Prognostic Value of FDG PET Quantitative Imaging Features Combined with Clinical Prognostic Factors". In: Radiology 278.1 (2016). Radiology, pp. 214-22. DOI: 10.1148/radiol.2015142920.

[27] T. P. Coroller et al. "Radiomic phenotype features predict pathological response in non-small cell lung cancer". In: Radiother Oncol 119.3 (2016). Radiotherapy and oncology : journal of the European Society for Therapeutic Radiology and Oncology, pp. 480-6. DOI: 10.1016/j.radonc.2016.04.004.

[28] S. Carvalho et al. "Early variation of FDG-PET radiomics features in NSCLC is related to overall survival the delta radiomics concept". In: Radiotherapy and Oncology 118 (Sept. 2018), S20-S21. ISSN: 0167-8140. DOI: 10 . 1016 / s0167 - 8140(16 ) 30042 - $1 . \quad$ URL: https://doi.org/10.1016/S0167-8140(16)30042-1.

[29] A. Cunliffe et al. "Lung texture in serial thoracic computed tomography scans: correlation of radiomics-based features with radiation therapy dose and radiation pneumonitis development". In: Int J Radiat Oncol Biol Phys 91.5 (2015). International journal of radiation oncology, biology, physics, pp. 1048-56. DOI: 10.1016/j.ijrobp.2014.11.030.

[30] A. K. Dhara et al. "A Combination of Shape and Texture Features for Classification of Pulmonary Nodules in Lung CT Images". In: J Digit Imaging 29.4 (2016). Journal of digital imaging, pp. 466-75. DOI: 10.1007/s10278-015-9857-6.

[31] S. K. Dilger et al. "Improved pulmonary nodule classification utilizing quantitative lung parenchyma features". In: J Med Imaging (Bellingham) 2.4 (2015). Journal of medical imaging (Bellingham, Wash.), p. 041004. DOI: 10.1117/1.jmi.2.4.041004.

[32] J. A. Oliver et al. "Variability of Image Features Computed from Conventional and Respiratory-Gated PET/CT Images of Lung Cancer". In: Transl Oncol 8.6 (2015). Translational oncology, pp. 524-34. DOI: 10.1016/j.tranon.2015.11.013.

[33] S. Yip et al. "Comparison of texture features derived from static and respiratorygated PET images in non-small cell lung cancer". In: PLoS One 9.12 (2014). PloS one, e115510. DOI: 10.1371/journal.pone.0115510.

[34] O. van Gomez Lopez et al. "Heterogeneity in [(1)(8)F]fluorodeoxyglucose positron emission tomography/computed tomography of non-small cell lung carcinoma and its relationship to metabolic parameters and pathologic staging". In: Mol Imaging 13 (2014). Molecular imaging. DOI: 10.2310/7290.2014.00032.

[35] S. Carvalho et al. "OC-0205: Prognostic value of pre-RT PET metrics of lymph nodes vs. primary tumor in NSCLC: which holds more information?" In: Radiotherapy and Oncology 115 (2015), S103-S104. DOI: 10.1016/S0167-8140(15)40203-8. URL: http://dx.doi.org/10.1016/S0167-8140(15)40203-8.

[36] C. M. Zegers et al. "In vivo quantification of hypoxic and metabolic status of NSCLC tumors using [18F]HX4 and [18F]FDG-PET/CT imaging". In: Clin Cancer Res 20.24 (2014). Clinical cancer research : an official journal of the American Association for Cancer Research, pp. 6389-97. DOI: 10.1158/1078-0432.ccr-14-1524.

[37] C. M. L. Zegers et al. "Hypoxia imaging with [(1)(8)F]HX4 PET in NSCLC patients: defining optimal imaging parameters." eng. In: Radiotherapy and oncology : journal of the European Society for Therapeutic Radiology and Oncology 109 (1 2013), pp. 58-64. 
[38] L. J. Dubois et al. "Preclinical evaluation and validation of [18F]HX4, a promising hypoxia marker for PET imaging". In: Proc Natl Acad Sci U S A 108.35 (2011). Proceedings of the National Academy of Sciences of the United States of America, pp. 14620-5. DOI: 10.1073/pnas.1102526108.

[39] J. van Loon et al. "PET imaging of hypoxia using [18F]HX4: a phase I trial". In: Eur J Nucl Med Mol Imaging 37.9 (2010). European journal of nuclear medicine and molecular imaging, pp. 1663-8. DOI: 10.1007/s00259-010-1437-x.

[40] P. E. Galavis et al. "Variability of textural features in FDG PET images due to different acquisition modes and reconstruction parameters". In: Acta Oncol 49.7 (2010). Acta oncologica (Stockholm, Sweden), pp. 1012-6. DOI: 10.3109/0284186x. 2010.498437.

[41] X. Fave et al. "Can radiomics features be reproducibly measured from CBCT images for patients with non-small cell lung cancer?" In: Med Phys 42.12 (2015). Medical physics, pp. 6784-97. DOI: 10.1118/1.4934826.

[42] R. T. Leijenaar et al. "The effect of SUV discretization in quantitative FDG-PET Radiomics: the need for standardized methodology in tumor texture analysis". In: Sci Rep 5 (2015). Scientific reports, p. 11075. DOI: 10.1038/srep11075.

[43] Y. Balagurunathan et al. "Test-retest reproducibility analysis of lung CT image features". In: J Digit Imaging 27.6 (2014). Journal of digital imaging, pp. 805-23. DOI: $10.1007 / \mathrm{s} 10278-014-9716-x$.

[44] C. C. Nagel et al. "Phased attenuation correction in respiration correlated computed tomography/positron emitted tomography". In: Med Phys 33.6 (2006). Medical physics, pp. 1840-7. DOI: 10.1118/1.2198170.

[45] W. van Elmpt et al. "Optimal gating compared to 3D and 4D PET reconstruction for characterization of lung tumours". In: Eur J Nucl Med Mol Imaging 38.5 (2011). European journal of nuclear medicine and molecular imaging, pp. 843-55. DOI: 10. 1007/s00259-010-1716-6.

[46] C. Liu et al. "Quiescent period respiratory gating for PET/CT". In: Med Phys 37.9 (2010). Medical physics, pp. 5037-43. DOI: 10.1118/1.3480508.

[47] M. Dawood et al. "Respiratory motion correction in 3-D PET data with advanced optical flow algorithms". In: IEEE Trans Med Imaging 27.8 (2008). IEEE transactions on medical imaging, pp. 1164-75. DOI: 10.1109/tmi.2008.918321.

[48] P. Lambin et al. "Predicting outcomes in radiation oncology-multifactorial decision support systems". In: Nat Rev Clin Oncol 10.1 (2013). Nature reviews. Clinical oncology, pp. 27-40. DOI: 10.1038/nrclinonc.2012.196.

[49] P. Lambin et al. "Rapid Learning health care in oncology an approach towards decision support systems enabling customised radiotherapy". In: Radiother Oncol 109.1 (2013). Radiotherapy and oncology : journal of the European Society for Therapeutic Radiology and Oncology, pp. 159-64. DOI: 10.1016/j.radonc.2013.07. 007.

[50] P. Lambin et al. "The ESTRO Breur Lecture 2009. From population to voxel-based radiotherapy: exploiting intra-tumour and intra-organ heterogeneity for advanced treatment of non-small cell lung cancer". In: Radiother Oncol 96.2 (2010). Radiotherapy and oncology : journal of the European Society for Therapeutic Radiology and Oncology, pp. 145-52. DOI: 10.1016/j.radonc.2010.07.001. 
[51] P. Lambin et al. "Modern clinical research: How rapid learning health care and cohort multiple randomised clinical trials complement traditional evidence based medicine". In: Acta Oncol 54.9 (2015). Acta oncologica (Stockholm, Sweden), pp. 1289-300. DOI: $10.3109 / 0284186 x .2015 .1062136$. 



\section{Methodological challenges}

in image feature studies 



\section{Chapter 3}

Quality assessment of positron emission tomography scans: recommendations for future multicenter trials

Evelyn E.C. de Jong, Wouter van Elmpt, Otto S. Hoekstra, Harry J.M. Groen, Egbert F. Smit, Ronald Boellaard, Philippe Lambin, Anne-Marie C. Dingemans 


\section{Abstract}

\section{Background}

Standardization protocols and guidelines for positron emission tomography (PET) in multicenter trials are available, despite a large variability in image acquisition and reconstruction parameters exist. In this study, we investigated the compliance of PET scans to the guidelines of the European Association of Nuclear Medicine (EANM). From these results, we provide recommendations for future multicenter studies using PET.

\section{Material and methods}

Patients included in a multicenter randomized phase II study had repeated PET scans for early response assessment. Relevant acquisition and reconstruction parameters were extracted from the digital imaging and communications in medicine (DICOM) header of the images. The PET image parameters were compared to the guidelines of the EANM for tumor imaging version 1.0 recommended parameters.

\section{Results}

From the 223 included patients, 167 baseline scans and 118 response scans were available from 15 hospitals. Scans of $19 \%$ of the patients had an uptake time that fulfilled the Uniform Protocols for Imaging in Clinical Trials response assessment criteria. The average quality score over all hospitals was $69 \%$. Scans with a non-compliant uptake time had a larger standard deviation of the mean standardized uptake value $\left(\mathrm{SUV}_{\text {mean }}\right)$ of the liver than scans with compliant uptake times.

\section{Conclusion}

Although a standardization protocol was agreed on, there was a large variability in imaging parameters. For future multicenter studies including PET imaging, a prospective central quality review during patient inclusion is needed to improve compliance with image standardization protocols as defined by EANM. 


\section{Background}

Many studies have shown that ${ }^{18} \mathrm{~F}$-fluorodeoxy glucose positron emission tomography $\left({ }^{18} \mathrm{~F}\right.$ FDG-PET) can be used for early response assessment $[1,2,3,4,5]$. Treatment response is measured by the relative change in standardized uptake value (SUV) during treatment. Many factors affect SUV, such as patient preparation procedures, acquisition parameters and reconstruction parameters. Therefore, if response assessment using ${ }^{18} \mathrm{~F}$-FDG-PET data is performed in multicenter studies it is of utmost importance that acquisition and reconstruction are standardized. Also in preclinical research the development of PET as a predictive tool is desirable. However, reaching a high degree of reproducibility and sensitivity in small animal studies using FDG-PET is also challenging. Therefore it is important to minimize methodological issues in preclinical as well as clinical studies as much as possible by standardize acquisition and reconstruction [6].

In 2010, the European Association of Nuclear Medicine (EANM) published the tumor PET imaging guidelines version 1.0, which give a minimum standard for acquisition and reconstruction of FDG-PET scans [7]. This protocol standardizes patient preparation, FDG dosage, reconstruction settings, data analysis and include a multi-center quality control phantom measurement procedure $[7,8,9,10]$.

In 2011, the multicenter randomized phase II NVALT12 (NCT01171170) study in stage IV non-small cell lung cancer started. Part of this study was an imaging sub-study where repeated ${ }^{18} \mathrm{~F}-\mathrm{FDG}$-PET imaging for early response assessment was performed [11]. In the NVALT12 protocol it was assumed that the EANM 1.0 imaging guidelines had been implemented in the hospitals and that ${ }^{18} \mathrm{~F}$-FDG-PET scans were performed according to these guidelines. EANM 1.0 reconstruction of the ${ }^{18} \mathrm{~F}-\mathrm{FDG}-\mathrm{PET}$ images was recommended, although reconstruction might be performed according to institutional standards and therefore variability in image parameters could be expected.

In this study, we investigated the quality of the ${ }^{18} \mathrm{~F}-\mathrm{FDG}-\mathrm{PET}$ scans in the NVALT12 study and the impact of deviations on the mean SUV of the liver. By identifying the sources of variability we derived recommendations for future reduction of these uncertainties for early response assessment.

\section{Material and methods}

Between January 2011 and January 2013, a total of 223 patients were included in the NVALT12 study [12]. The baseline ${ }^{18}$ F-FDG-PET scan was standard of care, and only for patients who had an ${ }^{18} \mathrm{~F}$-FDG-PET at baseline as part of the standard work-up for lung cancer a second ${ }^{18} \mathrm{~F}$-FDG-PET scan was performed. Scans were anonymized locally and per $\mathrm{CD}$ sent to the central hospital were the analysis was done. One hundred and sixty-seven baseline ${ }^{18} \mathrm{~F}$-FDG-PET scans and 118 response ${ }^{18} \mathrm{~F}$-FDG-PET scans were retrieved. Scans that could not be converted to SUV (i.e. SUV conversion information missing) were also excluded (baseline $n=9$; response $n=9$ ), which meant that only for 158 baseline scans and 109 response scans the SUV mean of the liver could be analyzed. The ${ }^{18}$ F-FDG-PET scans were obtained in a multicentric setting from 15 different hospitals using PET scanners from three different vendors: vendor $A$ (Philips Healthcare, Best, the Netherlands), vendor B (GE Healthcare, Chicago, IL, USA) and vendor C (Siemens Healthineers, Erlangen, Germany).

For the quality analysis, we performed a Digital Imaging and Communications in 
Medicine (DICOM) header extraction to extract the radiopharmaceutical start time, series time, attenuation correction method, randoms correction method, scatter correction method, reconstruction method, convolution kernel width and matrix size.

To check the quality of the ${ }^{18}$ F-FDG-PET scans, the image acquisition and reconstruction parameters extracted from the DICOM header were compared to the acquisition and reconstruction parameters as recommended by the EANM 1.0. In the paragraphs below the EANM parameters that were used for the quality check are described.

\section{Uptake time}

The EANM 1.0 guidelines recommend that emission scanning should start 55-65 minutes after FDG administration. To test if the scans of a patient could be used for response assessment the Uniform Protocol for Imaging in Clinical Trials (UPICT) criteria were used. The UPICT protocol specifies that when a scan is repeated on the same patient, it is important that the uptake time is consistent with an acceptable variance of \pm 15 minutes, provided that both scans do not start before 55 minutes after FDG administration [10]. The uptake time in our study was the difference in time between the radiopharmaceutical start time and the start of the PET acquisition (i.e. DICOM series time).

\section{Corrections}

In order to obtain the quantitative information from a ${ }^{18} \mathrm{~F}-\mathrm{FDG}-\mathrm{PET}$ scan, an attenuation correction and corrections for random and scatter coincidences are required [13]. Different methods to correct for randoms and scatter exist, depending on the specific implementation at the scanner and the vendor. For this analysis, it was only checked if the correction was applied.

\section{Image reconstruction}

Based on a phantom study in 2008, most PET/CT scanners provide images with a spatial resolution close to $7 \mathrm{~mm}$ full width at half maximum (FWHM) when using the default by the manufacturer-recommended image reconstruction settings [9]. Other settings, such as smoothing filters and image matrix size can be changed with preservation of a final image resolution of approximately $7 \mathrm{~mm}$ FWHM and harmonized interpretation and analysis of multicenter data is possible.

The scans in this study were performed on eight different PET scanners from three different vendors: $41 \%(69 / 167)$ of the scans were performed on a scanner of vendor $A$, $2 \%(4 / 167)$ on a scanner of vendor $B$ and $56 \%(94 / 167)$ on a scanner of vendor $C$. For scans performed on a scanner of vendor $\mathrm{B}$ or $\mathrm{C}$, the indicative reconstruction settings are a matrix size of $128 \times 128$ or $256 \times 256$, a post-reconstruction smoothing using a $5-6 \mathrm{~mm}$ FWHM Gaussian filter and a 2D/3D ordered subset expectation maximization (OSEM) reconstruction with the number of iterations times the number of subsets above 30 . For scans performed on a scanner of vendor $A$, the indicative reconstruction settings are a matrix size of $144 \times 144$ and a line-of-response (LOR-RAMLA)/3D RAMLA/3D orderedsubset iterative time-of-flight (BLOB-OS-TF) reconstruction method. For reconstructions with point spread function (PSF) corrections, the post-reconstruction smoothing need to be increased to $7-8 \mathrm{~mm}$. 


\section{Quality score}

To check possible differences in PET quality between the different hospitals we scored every scan individually and calculated the average score of the scans per hospital. For every correct parameter, the scan received a point and the number of points per scan were divided by the number of known parameters for that scan, so missing data were excluded from the quality score.

\section{Influence on SUV}

To investigate the influence of image parameters on SUV, a spherical volume of interest with a diameter of $3 \mathrm{~cm}$ was delineated in the right lobe of the liver [14]. A mean SUV of the liver between 1.3 and 2.5 was defined as a correct quality scan [8].

\section{Results}

There were only a few scans with differences in reconstruction parameters between the baseline scan and the response scan; some patients had the baseline scan on a scanner of vendor $C$ and the response scan on a scanner of vendor $A(n=2)$ and some other patients had the baseline scan without PSF and the response scan with PSF $(n=2)$ (see also Figure 3.1).

About one-third of the scans $(35 \% ; 97 / 280)$ of all scans had an uptake time between 55 and $65 \mathrm{~min}$. Twelve percent $(14 / 118)$ of the scans had an uptake time between 55 and $65 \mathrm{~min}$ for the baseline scan and for the response scan (Figure 3.2). Due to the range in uptake time we calculated the median instead of the mean. The median uptake time was $64 \mathrm{~min}$ for the baseline scan as well as for the response scan (see also Table 3.1 in the Supplementary Material). In 11 patients both scans had an uptake time less than 55 min. In only $19 \%(53 / 285)$ of the patients, both scans had an uptake time of more than 55 min and a difference of less than 15 min between the two scans and could therefore be used for response assessment according to UPICT.

The corrections for attenuation, random and scatter coincidences could be retrieved for $86 \%(144 / 167)$ of the baseline scans and for $78 \%(92 / 118)$ of the response scans (Table 3.1 in the Supplementary Material). For the other scans the information was missing. Scans that had missing information were from the same hospital (see also Figure 3.1).

About $50 \%$ of the baseline scans and $50 \%$ of the response scans performed on a scanner of vendor A were reconstructed according to the EANM 1.0 guidelines. For $24 \%$ of the baseline scans and $25 \%$ of the response scans performed on a scanner of vendor $A$ the reconstruction method was missing. For the scans performed on a scanner of vendor $\mathrm{B}$ or $\mathrm{C}$ a large variability in reconstruction method existed. Thirty-three percent of the baseline scans and $37 \%$ of the response scans performed on a scanner of vendor $\mathrm{B}$ or $\mathrm{C}$ were reconstructed according to the EANM 1.0 guidelines. No reconstruction parameters were missing for the scans performed on a scanner of vendor B or $C$.

The average quality score over all hospitals was 0.69 (range $0.46-0.88$ ), which means that on average more than two-third of the image parameters were EANM 1.0 guideline compliant. Hospitals with a scanner of vendor A scored on average slightly higher than hospitals with a scanner of vendor $B$ or $C$ (vendor $A$ : 0.80 (range: $0.56-0.88$ ); vendor $B$ or C: $0.64(0.46-0.88))$. 
Although there was a large variability in image parameters, most scans had a SUV mean $_{\text {man }}$ of the liver which was EANM 1.0 guideline compliant (64\%; 171/267), only $5 \%(8 / 158)$ of the baseline scans and $2 \%(2 / 109)$ of the response scans had a SUV $\mathrm{Sman}_{\text {mean }}$ below 1.3. Twenty percent $(53 / 267)$ of the scans had a SUV mean of the liver as well as an uptake time that was EANM 1.0 guideline compliant (Figure 3.4 in the Supplementary Material). There was no difference in mean SUV $_{\text {mean }}$ of the liver for baseline or response scans with an EANM 1.0 or UPICT compliant uptake time. When looking at the scans with a nonguideline compliant uptake time, the mean SUV $_{\text {mean }}$ of the liver is comparable 2.33 for the baseline scan and 2.23 for the response scan although the standard deviation is slightly increased 0.62 for the baseline scan and 0.49 for the response scan.

In Figure 3.3, the hospitals were ranked in descending order according to their quality score and there seems no trend between the quality score of the hospital and the variability in $S U V_{\text {mean }}$ of the liver. No difference in $S U V_{\text {mean }}$ of the liver was found for the different vendors.

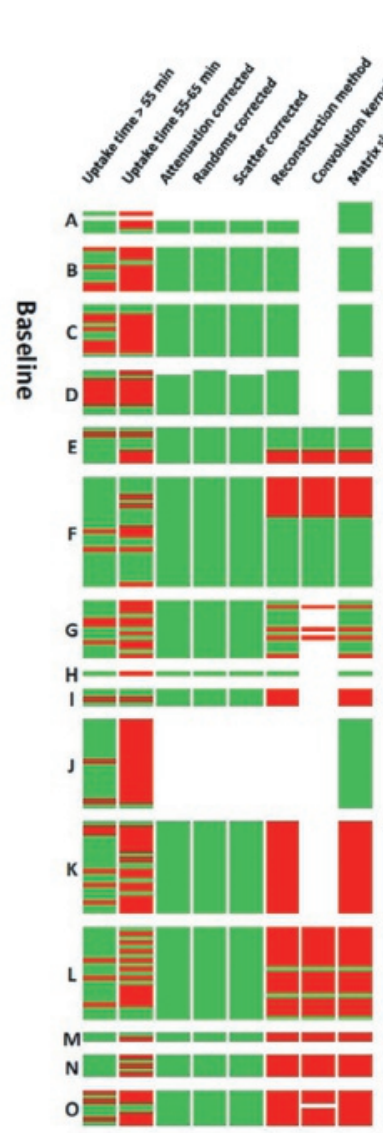

a)

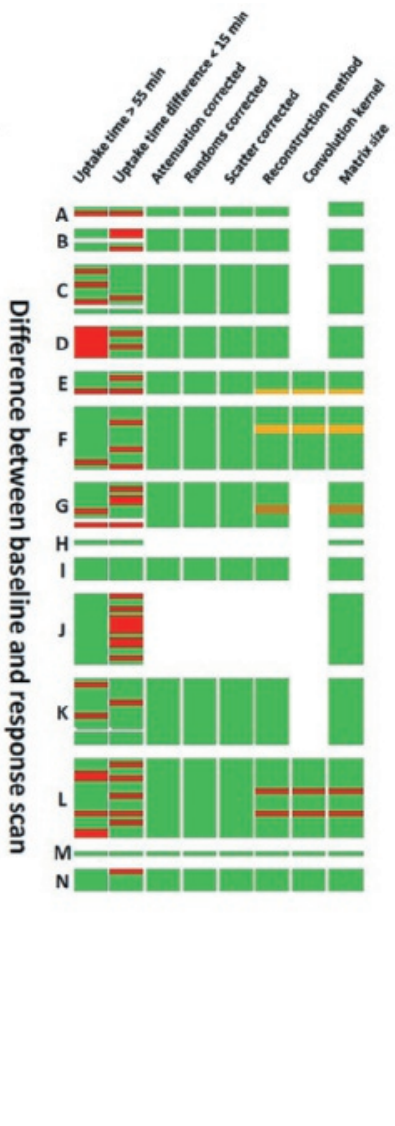

b)
Figure 3.1: (A) Heatmap of the image parameters of the baseline scan ranked in descending order according to the quality score of the hospital. (B) Heatmap of the difference in image parameters between the baseline scan and the response scan ranked in descending order according to the quality score of the hospital. Green: EANM guideline compliant, white: missing information, orange: improved for response scan, red: EANM guideline non-compliant/worsened

for response scan, striped: response scan on different scanner than baseline scan. 


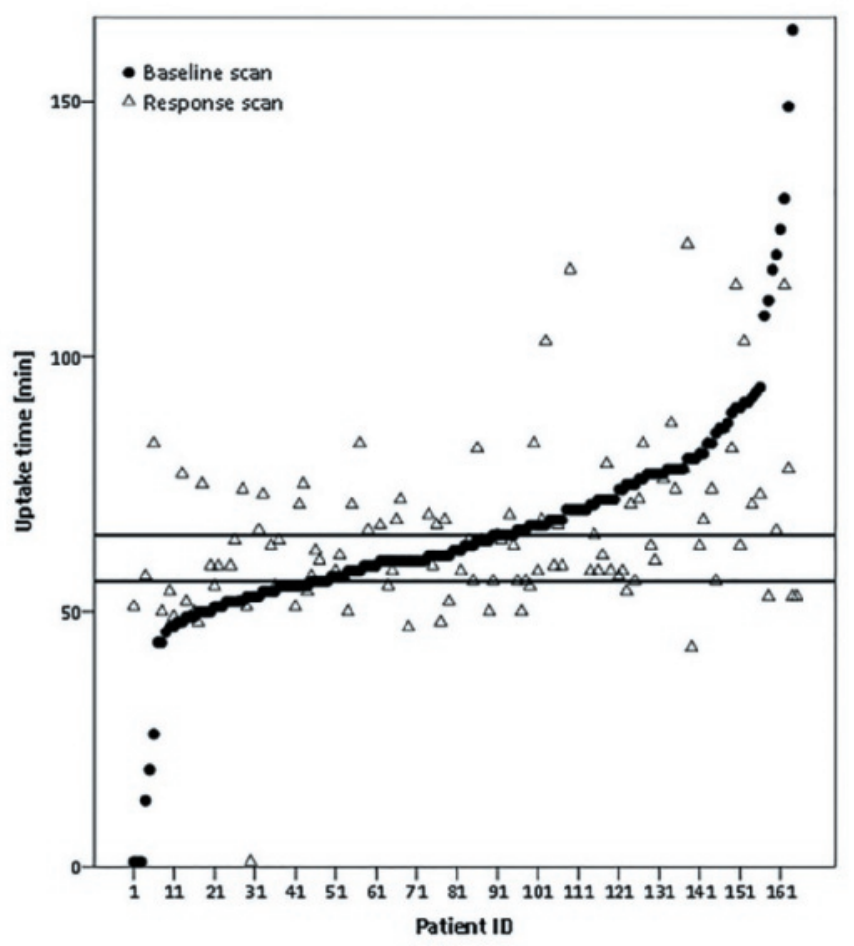

Figure 3.2: Time interval between injection and start time of scan per patient ranked in ascending order for the baseline scan. The horizontal lines show the NEDPAS specified maximum (65) and minimal $(55)$ uptake times. One outlier $(106 ; 251)$ is removed for visualization purposes.

\section{Discussion}

Despite the availability of imaging guidelines for ${ }^{18}$ F-FDG-PET [7, 8, 9, 10, 15], our analysis shows that a large variability in image acquisition and reconstruction parameters existed in the ${ }^{18} \mathrm{~F}$-FDG-PET scans of the multicenter NVALT12 trial. In this study, we investigated the number of ${ }^{18} \mathrm{~F}$-FDG-PET scans of the NVALT12 study that were EANM 1.0 guideline compliant and the impact of deviations on SUV. Next to that, we speculate about possible reasons for deviations from this guideline.

The EANM 1.0 guideline recommend that PET imaging takes place $60 \pm 5$ min after administration of FDG [9]. This uptake time window is essentially empiric and determined taken financial considerations into account, and probably not optimal [15]. It is well known that lesion SUV for FDG continues to rise for at least $45 \mathrm{~min}$ after FDG injection, reaching a plateau between 60 and 90 minutes $[16,17]$. In our study, only $34 \%$ of all scans had an uptake time as recommended by the EANM 1.0. With that we show that in clinical practice it is hard to have an uptake time within the recommended window of $10 \mathrm{~min}$. In the EANM guidelines version 2.0, this is changed to an acceptable uptake time window of $55-75 \mathrm{~min}, 59 \%$ of the scans in this study had an uptake time within this window. Another possible reason for deviations is that the radiopharmaceutical start time extracted 


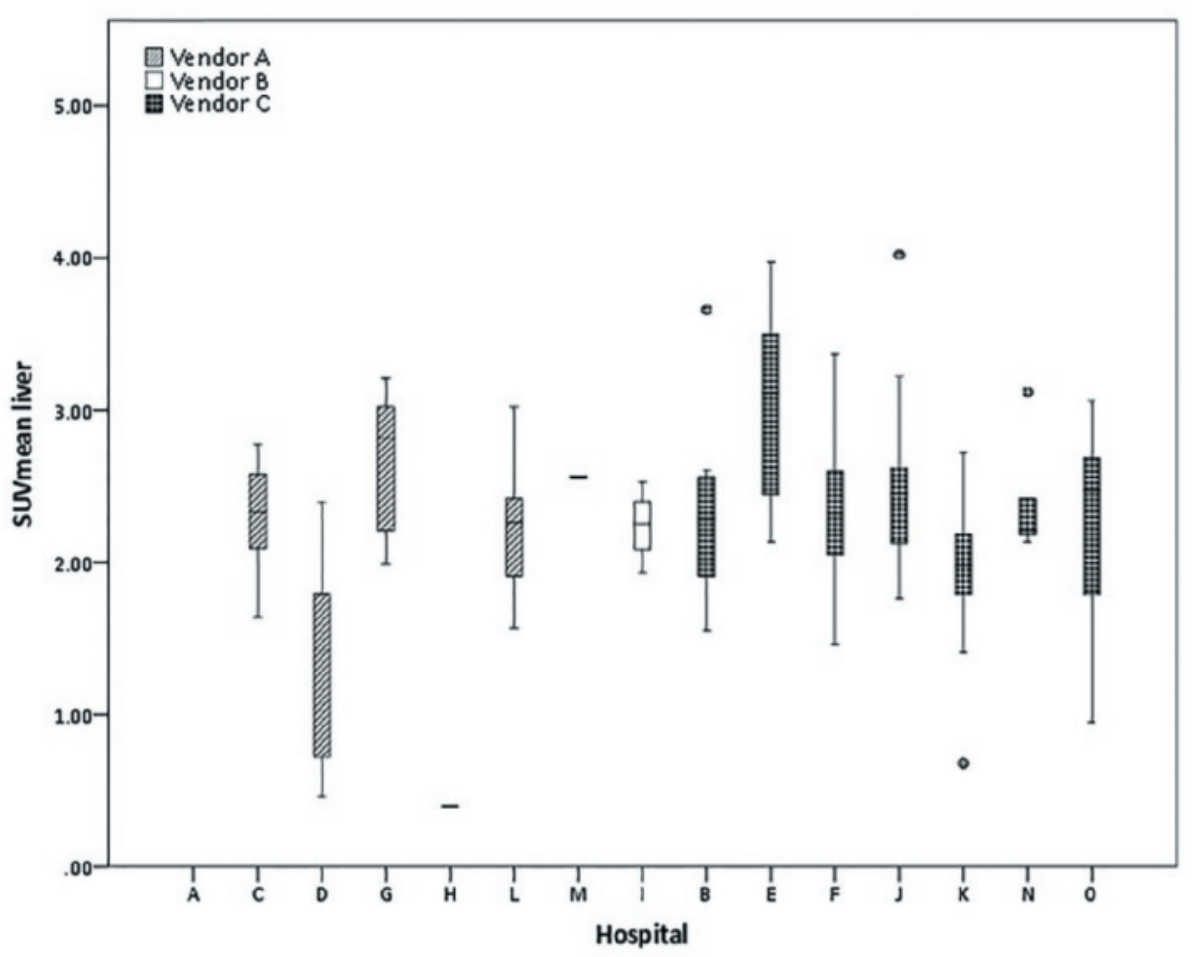

Figure 3.3: Boxplot of $\mathrm{SUV}_{\text {mean }}$ of the liver of the baseline scan ranked in descending order according to the quality score and per vendor, the scans of hospital A were, due to a missing SUV conversion tag, not converted to SUV and are therefore empty in the graph.

from the DICOM header was not the time of FDG administration as we assumed. Because it is currently not possible to separately store information on injection time and calibration time in the DICOM header, some ambiguity which time is in the DICOM header under the name radiopharmaceutical start time exists [18].

Reconstruction algorithms and voxel size are different for the various available PET systems and vendor dependent. Therefore, it is a challenge to ensure that images of PET scanners from different vendors are quantitatively comparable [15]. Multiple studies have shown that liver metabolism can be used as reference organ due to its stable uptake over time and therefore suitable for assessment of scan parameters [18]. We therefore used the SUV mean of liver as quality control measure. However, our results did not show a relation between the quality score and $S_{U} V_{\text {mean }}$ of the liver. Kuhnert et al. [19] showed that for parameters other than reconstruction method and settings (injected activity and uptake time for example), the $\mathrm{SUV}_{\text {mean }}$ of the liver could be used for quality assessment in compliance with the EANM guideline, meaning that the large variability in SUV mean of the liver is most probably caused by the differences in uptake time and net injected activity. We showed that like Hristova et al. [18] the variability in SUV mean of the liver is slightly smaller for the scans with a guideline compliant uptake time than for the scans 
with non-compliant uptake times (SUV mean $2.29 \pm 0.46$ vs. $2.33 \pm 0.62)$.

The hospital analysis showed that the average score over all hospitals is 0.69 and that scans from the same hospital mostly have the same non-compliant image parameters. Scans with missing parameters are typically from the same hospitals which is most probably caused by the anonymization procedure.

Our study shows that in a multicenter trial still a large variability in image parameters exist. Especially when using the PET information for treatment decisions, like in dose painting the high uptake region [20], it is important that acquisition and reconstruction are standardized, otherwise different sub-volumes will be boosted. When more complex analysis methods (e.g., Radiomics) are used to predict therapy response using PET in the future, it is important that image acquisition and reconstruction are standardized [21]. A study of Carvalho et al. [22] investigated next to common PET descriptors like SUV $\max$ and $S_{U} U V_{\text {peak }}$ if more complex descriptors like intensity volume histograms of the PET signal are prognostic in NSCLC. They only found a trend that patients with tumors with a more homogeneous distribution of SUV had a better prognosis [22]. Although they only had data from one hospital, the image protocol changed during inclusion criteria and therefore variability in SUV could be present what could have a caused the non-significance of $\mathrm{SUV}_{\max }$ for prognosis.

A few recommendations for future multicenter studies with repeated PET imaging are drawn. For drafting the study protocol, it is recommended to collaborate with radiologists and nuclear medicine experts to assure access to the current imaging guidelines to keep the variability in image acquisition and reconstruction parameters as low as possible. Next, it is recommended to use an anonymization process that keeps all DICOM fields necessary for SUV quantification and investigation of the acquisition and reconstruction parameters. For calculating the uptake time, take the acquisition time near the midplane slice of the tumor. A final recommendation in a multicenter trial setting using ${ }^{18} \mathrm{~F}-\mathrm{FDG}$-PET involves a prospective rapid quality review, which is a good procedure to improve compliance with imaging guidelines and tackle difficulties in image sharing and processing as early as possible [18]. More details of these recommendations can be found in Table 3.2 in the Supplementary Material.

In conclusion, although guidelines for tumor imaging exist, there is still a large variability in acquisition and reconstruction parameters of ${ }^{18} \mathrm{~F}-\mathrm{FDG}-\mathrm{PET}$ imaging in multicenter studies. In the future, these variations should be minimized by performing a rapid quality review that tackle difficulties in image sharing and processing as early as possible. 


\section{References}

[1] W. van Elmpt et al. "Response assessment using 18F-FDG PET early in the course of radiotherapy correlates with survival in advanced-stage non-small cell lung cancer". In: J Nucl Med 53.10 (2012). Journal of nuclear medicine : official publication, Society of Nuclear Medicine, pp. 1514-20. DOI: 10.2967/jnumed.111.102566.

[2] L. F. de Geus-Oei et al. "Chemotherapy response evaluation with 18F-FDG PET in patients with non-small cell lung cancer". In: J Nucl Med 48.10 (2007). Journal of nuclear medicine : official publication, Society of Nuclear Medicine, pp. 1592-8. DOI: 10.2967/jnumed.107.043414. URL: http://www.ncbi.nlm.nih.gov/pubmed/ 17873138.

[3] A. M. Dingemans et al. "First-line erlotinib and bevacizumab in patients with locally advanced and/or metastatic non-small-cell lung cancer: a phase II study including molecular imaging". In: Ann Oncol 22.3 (2011). Annals of oncology : official journal of the European Society for Medical Oncology / ESMO, pp. 559-66. DOI: 10.1093/ annonc/mdq391. URL: http://www.ncbi.nlm.nih.gov/pubmed/20702788.

[4] R. K. Ramanathan et al. "Positron emission tomography response evaluation from a randomized phase III trial of weekly nab-paclitaxel plus gemcitabine versus gemcitabine alone for patients with metastatic adenocarcinoma of the pancreas". In: Ann Oncol 27.4 (2016). Annals of oncology : official journal of the European Society for Medical Oncology, pp. 648-53. DOI: 10.1093/annonc/mdw020.

[5] A. Hendlisz et al. "Serial FDG-PET/CT for early outcome prediction in patients with metastatic colorectal cancer undergoing chemotherapy". In: Ann Oncol 23.7 (2012). Annals of oncology : official journal of the European Society for Medical Oncology, pp. 1687-93. DOI: 10.1093/annonc/mdr554.

[6] M. Busk et al. "FDG-PET reproducibility in tumor-bearing mice: comparing a traditional SUV approach with a tumor-to-brain tissue ratio approach". In: Acta Oncol (2017). Acta oncologica (Stockholm, Sweden), pp. 1-10. DOI: 10.1080/0284186x.2016.1276620.

[7] R. Boellaard et al. "FDG PET and PET/CT: EANM procedure guidelines for tumour PET imaging: version 1.0". In: Eur J Nucl Med Mol Imaging 37.1 (2010). European journal of nuclear medicine and molecular imaging, pp. 181-200. DOI: 10.1007/ s00259-009-1297-4.

[8] R. Boellaard et al. "FDG PET/CT: EANM procedure guidelines for tumour imaging: version 2.0". In: Eur J Nucl Med Mol Imaging 42.2 (2015). European journal of nuclear medicine and molecular imaging, pp. 328-54. DOI: 10.1007/s00259-0142961-x. URL: http://www.ncbi.nlm.nih.gov/pubmed/25452219.

[9] R. Boellaard et al. "The Netherlands protocol for standardisation and quantification of FDG whole body PET studies in multi-centre trials". In: Eur J Nucl Med Mol Imaging 35.12 (2008). European journal of nuclear medicine and molecular imaging, pp. 2320-33. DOI: 10.1007/s00259-008-0874-2. URL: http://www.ncbi.nlm.nih. gov/pubmed/18704407.

[10] M. M. Graham et al. "Summary of the UPICT Protocol for 18F-FDG PET/CT Imaging in Oncology Clinical Trials". In: J Nucl Med 56.6 (2015). Journal of nuclear medicine : official publication, Society of Nuclear Medicine, pp. 955-61. DOI: 10. 2967/jnumed.115.158402. 
[11] E. E. de Jong et al. “[18F]FDG PET/CT-based response assessment of stage IV non-small cell lung cancer treated with paclitaxel-carboplatin-bevacizumab with or without nitroglycerin patches". In: Eur J Nucl Med Mol Imaging 44.1 (2017). European journal of nuclear medicine and molecular imaging, pp. 8-16. DOI: 10.1007/s00259-016-3498-y.

[12] A. M. Dingemans et al. "A randomized phase II study comparing paclitaxel-carboplatin-bevacizumab with or without nitroglycerin patches in patients with stage IV nonsquamous nonsmall-cell lung cancer: NVALT12 (NCT01171170)". In: Ann Oncol 26.11 (2015). Annals of oncology : official journal of the European Society for Medical Oncology, pp. 2286-93. DOI: $10.1093 /$ annonc/mdv370.

[13] J. L. Herraiz and A. Sitek. "Sensitivity estimation in time-of-flight list-mode positron emission tomography". In: Med Phys 42.11 (2015). Medical physics, pp. 6690-702. DOI: $10.1118 / 1.4934374$.

[14] R. L. Wahl et al. "From RECIST to PERCIST: Evolving Considerations for PET response criteria in solid tumors". In: J Nucl Med 50 Suppl 1 (2009). Journal of nuclear medicine : official publication, Society of Nuclear Medicine, 122S-50S. DOI: 10.2967 / jnumed.108.057307. URL: http: / / www . ncbi . nlm . nih . gov/pubmed/ 19403881.

[15] M. M. Graham, R. D. Badawi, and R. L. Wahl. "Variations in PET/CT methodology for oncologic imaging at U.S. academic medical centers: an imaging response assessment team survey". In: J Nucl Med 52.2 (2011). Journal of nuclear medicine : official publication, Society of Nuclear Medicine, pp. 311-7. DOI: 10.2967/jnumed.109.074104.

[16] S. Beaulieu et al. "SUV varies with time after injection in (18)F-FDG PET of breast cancer: characterization and method to adjust for time differences". In: J Nucl Med 44.7 (2003). Journal of nuclear medicine : official publication, Society of Nuclear Medicine, pp. 1044-50.

[17] D. S. Binns et al. "Compliance with PET acquisition protocols for therapeutic monitoring of erlotinib therapy in an international trial for patients with non-small cell lung cancer". In: Eur J Nucl Med Mol Imaging 38.4 (2011). European journal of nuclear medicine and molecular imaging, pp. 642-50. DOI: 10.1007/s00259-010-1665-0.

[18] I. Hristova et al. "Retrospective quality control review of FDG scans in the imaging sub-study of PALETTE EORTC 62072/VEG110727: a randomized, double-blind, placebo-controlled phase III trial". In: Eur J Nucl Med Mol Imaging 42.6 (2015). European journal of nuclear medicine and molecular imaging, pp. 848-57. DOI: 10. 1007/s00259-015-3002-0.

[19] G. Kuhnert et al. "Impact of PET/CT image reconstruction methods and liver uptake normalization strategies on quantitative image analysis". In: Eur J Nucl Med Mol Imaging 43.2 (2016). European journal of nuclear medicine and molecular imaging, pp. 249-58. DOI: 10.1007/s00259-015-3165-8.

[20] D. Di Perri et al. "Evolution of [18F]fluorodeoxyglucose and [18F]fluoroazomycin arabinoside PET uptake distributions in lung tumours during radiation therapy". In: Acta Oncol 56.4 (2017). Acta oncologica (Stockholm, Sweden), pp. 516-524. DOI: 10.1080/0284186x.2017.1287943. 
[21] R. T. Larue et al. "Quantitative radiomics studies for tissue characterization: a review of technology and methodological procedures". In: Br J Radiol 90.1070 (2017). The British journal of radiology, p. 20160665. DOI: 10.1259/bjr.20160665.

[22] S. Carvalho et al. "Prognostic value of metabolic metrics extracted from baseline positron emission tomography images in non-small cell lung cancer". In: Acta Oncol 52.7 (2013). Acta oncologica (Stockholm, Sweden), pp. 1398-404. DOI: 10.3109/ $0284186 x .2013 .812795$. 


\section{Supplementary Material}

\begin{tabular}{lllll}
\hline & & Baseline scan & Response scan & $\begin{array}{l}\text { Overlap baseline } \\
\text { and response }\end{array}$ \\
\hline Analyzed ${ }^{18}$ F-FDG-PET/CT scans & & 167 & 118 & 118 \\
\hline Time interval injection-scan & & Median(range) & Median(range) & \\
\hline & 555 min & $50(1-54)$ & $51(1-54)$ & \\
& $>65$ min & $60(55-64)$ & $59(55-64)$ & \\
\hline Time interval injection-scan & & $\% 65-164)$ & $74(65-251)$ & \\
\hline & $55-65$ min & $32 \%(53)$ & $37 \%(44)$ & $12 \%(14)$ \\
Attenuation correction & missing & $2 \%(3)$ & $2 \%(2)$ & $2 \%(2)$ \\
Randoms correction & yes & $86 \%(144)$ & $83 \%(98)$ & $86 \%(101)$ \\
Scatter correction & missing & $14 \%(23)$ & $17 \%(20)$ & $14 \%(17)$ \\
Reconstruction method & yes & $86 \%(144)$ & $83 \%(98)$ & $85 \%(100)$ \\
& missing & $14 \%(23)$ & $17 \%(20)$ & $15 \%(18)$ \\
Convolution kernel & yes & $86 \%(144)$ & $83 \%(98)$ & $86 \%(101)$ \\
& missing & $14 \%(23)$ & $17 \%(20)$ & $14 \%(17)$ \\
Matrix size & correct & $44 \%(74)$ & $44 \%(52)$ & $42 \%(50)$ \\
& missing & $14 \%(24)$ & $18 \%(21)$ & $16 \%(19)$ \\
& correct & $14 \%(24)$ & $14 \%(16)$ & $12 \%(14)$ \\
& missing & $57 \%(96)$ & $64 \%(75)$ & $62 \%(73)$ \\
& correct & $44 \%(74)$ & $59 \%(70)$ & $58 \%(68)$ \\
\hline
\end{tabular}

Table 3.1: Results of the quality analysis per scan. Correct means EANM guideline compliant; missing means not in DIOCM header; yes means correction is applied.

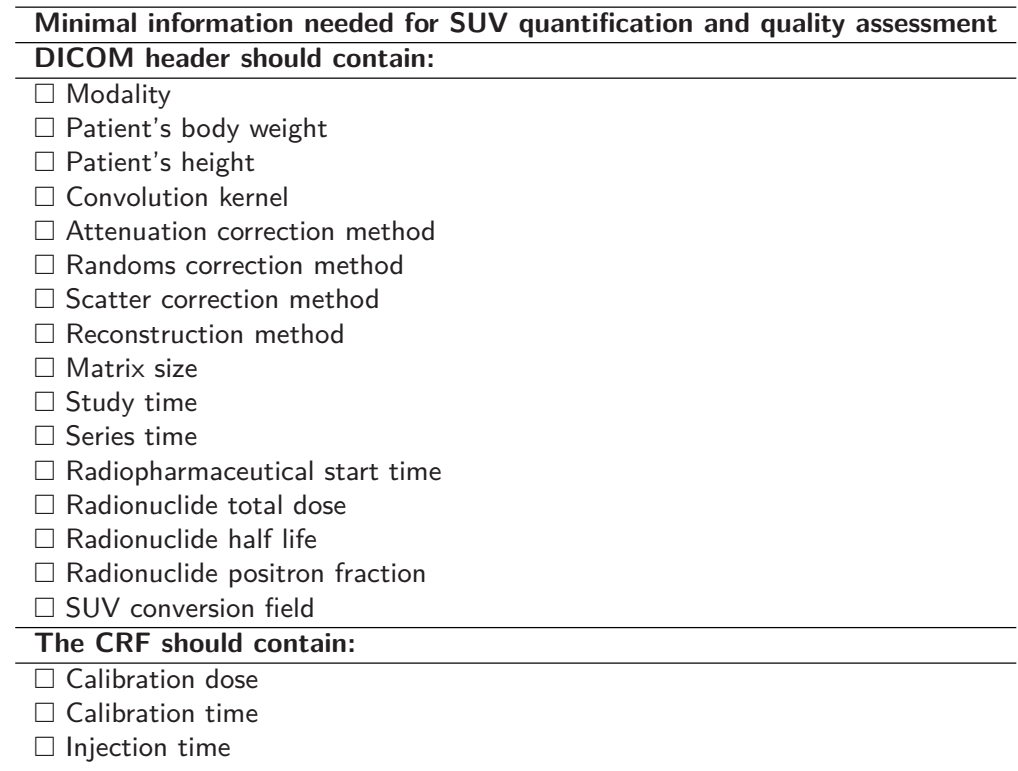

Table 3.2: Minimal information in DICOM header and Case Report Form that is needed for SUV quantification and quality assesment. 


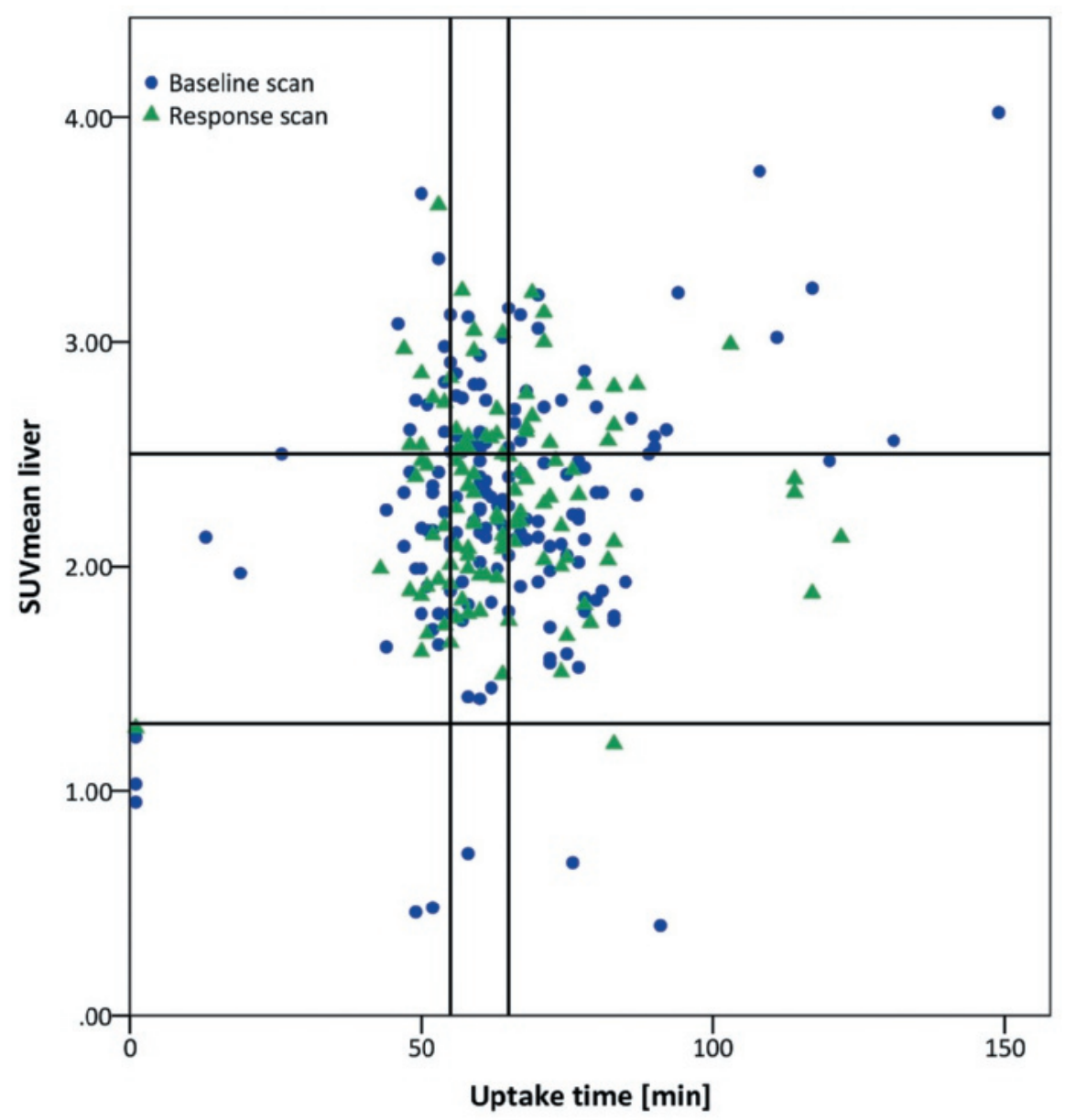

Figure 3.4: Uptake time versus the SUV mean of the liver. The horizontal lines show the by EANM specified liver SUV mean (1.3-2.5), the vertical lines show the by the EANM specified uptake time interval (55-65 min). (x-and y-axis shortened, two outliers missing $(251,2.09 ; 164,2.20)$ ). 



\section{Chapter 4}

What you see is (not) what you get: tools for a nonradiologist to evaluate image quality in lung cancer

Authors

Evelyn E.C. de Jong*, Lizza E.L. Hendriks*, Wouter van Elmpt, Hester A. Gietema, Paul A.M. Hofman, Dirk K.M. De Ruysscher, Anne-Marie C. Dingemans

\section{Adapted from}

Lung Cancer, 2018 September; 123: 112-115

DOI: 10.1016/j.lungcan.2018.07.014

*equal contribution 


\begin{abstract}
Medical images are an integral part of oncological patient records and they are reviewed by many different specialists. Therefore, it is important that besides imaging experts, other clinicians are also aware that the diagnostic value of a scan is influenced by the applied imaging protocol.

Based on two clinical lung cancer trials, we experienced that, even within a study protocol, there is a large variability in imaging parameters, which has direct impact on the interpretation of the image. These two trials were: 1 ) the NTR3628 in which the added value of gadolinium magnetic resonance imaging (Gd-MRI) to dedicated contrast enhanced computed tomography (CE-CT) for detecting asymptomatic brain metastases in stage III non-small cell lung cancer (NSCLC) was investigated and 2) a sub-study of the NVALT 12 trial (NCT01171170) in which repeated ${ }^{18} \mathrm{~F}$-fludeoxyglucose positron emission tomography ( $\left.{ }^{18} \mathrm{~F}-\mathrm{FDG}-\mathrm{PET}\right)$ imaging for early response assessment was investigated.

Based on the problems encountered in the two trials, we provide recommendations for non-radiology clinicians, which can be used in daily interpretation of imaging. Variations in image parameters cannot only influence trial results, but sub-optimal imaging can also influence treatment decisions in daily lung cancer care, when a physician is not aware of the scanning details.
\end{abstract}




\section{Introduction}

Medical imaging is an essential component of the diagnostic procedures performed in lung cancer. Next to that, it is also used for response assessment. The imaging modalities used in oncology have evolved from simple X-rays to computed tomography (CT)- and magnetic resonance imaging (MRI) scans. Nuclear imaging has innovated by the introduction of positron-emission tomography (PET) with several tracers being ${ }^{18}$ F-fludeoxyglucose $\left({ }^{18} \mathrm{~F}-\mathrm{FDG}\right)$ most frequently used. An ${ }^{18} \mathrm{~F}-\mathrm{FDG}-\mathrm{PET}$-scan using an integrated PET-CT scanner combined with a contrast enhanced CT is nowadays a standard staging technique in thoracic oncology. Due to ongoing technological innovations, the sensitivity and specificity of these modalities have significantly improved. However, many factors, as patient preparation, image acquisition and reconstruction parameters affect the quality and accuracy of all these exams [1].

Images are nowadays an integral part of electronic patient records and can be reviewed directly by many different health care specialists. Consequently, it is important that besides the imaging experts (i.e. radiologists and nuclear medicine physicians), other reviewing clinicians are aware that the diagnostic value of a scan is influenced by the applied imaging protocol and can recognize common artifacts (e.g. breathing artifacts). More knowledge on this topic will provide clinicians tools to communicate with their imaging colleagues to prevent image misinterpretation and to select the appropriate image acquisition protocol.

In two recently performed clinical trials (NTR3628 and NCT01171170) studying patients with non-small cell lung cancer (NSCLC) we experienced that, even though specific imaging guidelines were mandated by the trial protocol, there was a large variability in imaging parameters. This influenced not only the outcome of the trial but could also have impact on treatment by their clinical physician (medical oncologist / pulmonologist). In this manuscript, we describe the imaging problems encountered in these two trials. Furthermore, we will provide recommendations to guide clinicians in the interpretation of medical imaging based on our experience. Our goal is that this will result in improved clinical care as well as imaging standardization, not only in future multicenter studies, but also in daily clinical care.

\section{Methods}

\section{NVALT12 ${ }^{18}$ F-FDG-PET/CT imaging sub-study}

In all lung cancer patients eligible for therapy with curative intent, not only a chest CE$\mathrm{CT}$, but also a whole body ${ }^{18} \mathrm{~F}-\mathrm{FDG}-\mathrm{PET}$ is recommended $[2,3]$. The ${ }^{18} \mathrm{~F}-\mathrm{FDG}-\mathrm{PET}$, performed with a non-diagnostic low dose CT (LD-CT) for attenuation correction can be extended by an additional diagnostic CE-CT of the chest (with or without the upper abdomen and brain).

In the multicenter randomized phase II NVALT12 trial (NCT01171170) chemo-naïve patients with stage IV non-squamous NSCLC were treated with paclitaxel-carboplatinbevacizumab with or without nitroglycerin patches. For patients in whom an ${ }^{18} \mathrm{~F}$-FDG-PET at baseline was performed as part of standard work-up, a second study ${ }^{18}$ F-FDG-PET was performed within three weeks after start of treatment. The two ${ }^{18} \mathrm{~F}-\mathrm{FDG}$-PETs were used for response assessment, and results have been reported [4]. It was assumed that image acquisition was per the European Association of Nuclear Medicine (EANM) guidelines for 
tumor imaging version 1 as introduced in 2009, therefore no PET guidelines were added to the protocol [1].

\section{Study NTR3628: brain imaging}

Although patients with brain metastases often have neurological complaints, 3-21 \% of neurologically asymptomatic patients with otherwise stage I-III lung cancer are diagnosed with brain metastases on MRI [5]. This diagnosis is especially important in patients that are potentially eligible for therapy with curative intent. A post-gadolinium-MRI (post-Gd$\mathrm{MRI}$ ) is the imaging modality of choice, but when MRI is contra-indicated or too difficult to arrange within a reasonable time scale, a diagnostic CE-CT is an acceptable alternative [2, 3]. Except for the recommendation to include Gd-contrast series, no recommendations are given in the ESMO and NCCN lung cancer guidelines regarding the minimal requirements for this brain MRI (e.g. applied MRI sequences (e.g. T1, T2 FLAIR, diffusion weighted imaging) and minimum contrast amount) [2,3]. For brain CTs, intravenous administration of iodine-containing contrast is advised but otherwise no recommendations are made (e.g. minimum number of $\mathrm{mAs}$ and minimum contrast dose) [2,3].

In the multicenter NTR3628 study, the additional value of a post-contrast brain MRI was evaluated in stage III (based on ${ }^{18} \mathrm{~F}-\mathrm{FDG}-\mathrm{PET} / \mathrm{CE}-\mathrm{CT}$ ) NSCLC patients. All patients underwent a dedicated brain CE-CT as part of the staging whole body ${ }^{18}$ F-FDG-PET as standard of care [6]. Imaging requirements were: a standard ${ }^{18} \mathrm{~F}-\mathrm{FDG}-\mathrm{PET} / \mathrm{CE}-\mathrm{CT}$ protocol that included a diagnostic CE-CT brain, and a $1.5 \mathrm{~T}$ Gd-MRI brain (1 mm slices, $0.1 \mathrm{mmol} / \mathrm{kg}$ gadolinium), with a magnetization transfer contrast (MTC) pre-pulse to increase sensitivity and an additional post-contrast FLAIR sequence. MRI parameters were as recommended by an experienced neuro-radiologist $(\mathrm{PH})$ and followed the American College of Radiology Appropriateness Criteria (ACR AC) [7]. After inclusion of all patients, CE-CTs and MRIs were per protocol centrally reviewed by $\mathrm{PH}$ for protocol adherence and presence of brain metastases.

\section{Results}

\section{NVALT12 ${ }^{18}$ F-FDG-PET/CT imaging sub-study}

In the imaging analysis sub-study of the NVALT12, 167 baseline scans and 118 follow-up PET scans for response evaluation were present. Only 97 (34 \%) of the $285{ }^{18} \mathrm{~F}-\mathrm{FDG}-$ PETs performed in this study had an ${ }^{18} \mathrm{~F}-\mathrm{FDG}$ uptake time as recommended by the EANM. Fifty-four $(19 \%)$ scans had both uptake times in agreement with the uniform protocols for imaging in clinical trials (UPICT) guidelines. Supplementary Material Figure 4.2 shows the uptake times of the baseline- and the response scans ranked in ascending order for the baseline scan, only the uptake times between the red lines can be used for response assessment. The other investigated parameters in this imaging sub-study, correction factors (attenuation, randoms, scatter) and reconstruction settings (method, convolution kernel, matrix size) were assessed in a quality score.

In this study, $60 \%(171 / 285)$ of the ${ }^{18} \mathrm{~F}$-FDG-PETs had a SUV mean of the liver within reference values. Figure 4.1 shows an example of how a difference in SUV $_{\text {mean }}$ of the liver between the baseline and the response scan can lead to a visual underestimation of tumor response. Although we expected a non-compliant $\mathrm{SUV}_{\text {mean }}$ of the liver for scans with a 


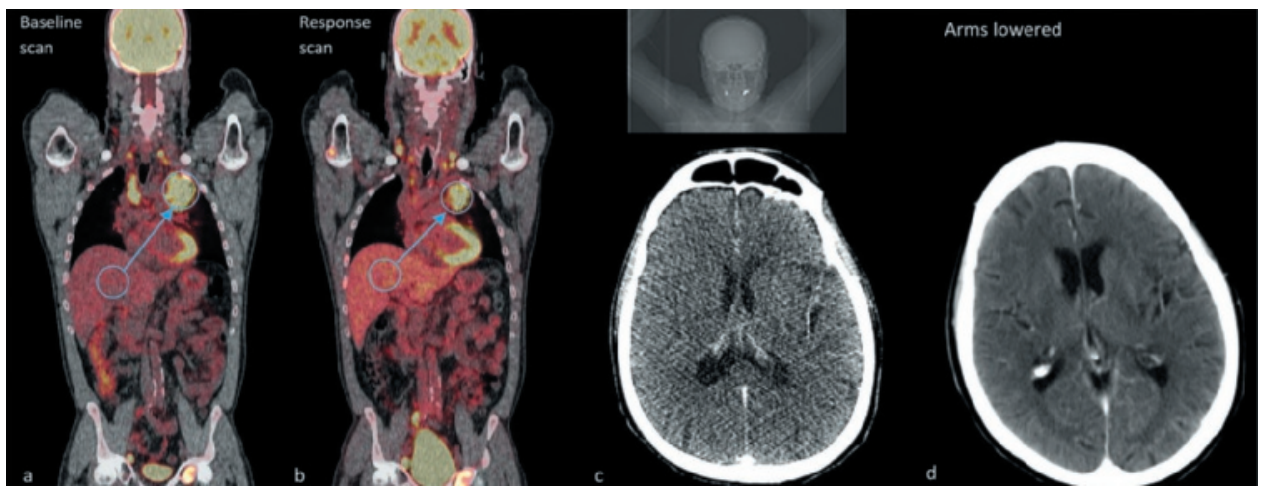

Figure 4.1: a) Baseline ${ }^{18} \mathrm{~F}$-FDG-PET/CT scan of the NCT01171170 trial; b) Response ${ }^{18} \mathrm{~F}-\mathrm{FDG}$ $\mathrm{PET} / \mathrm{CT}$ scan of the same patient, showing that a different $\mathrm{SUV}_{\text {mean }}$ of the liver can lead to a visually underestimation of response in the tumor; c) Axial CT image of the brain of the NTR3628 after contrast administration, reconstructed with a field of view of $500 \times 500 \mathrm{~mm}$ with raised arms (note the high level of noise and the streak artifacts due to beam hardening); d) Axial CT image of the brain of the same patient at approximate the same level, also after contrast administration, reconstructed with a FOV of $200 \times 200 \mathrm{~mm}$ with the arms lowered. The window and level setting are identical as well as other acquisition parameters for all imaging, note the difference in image quality.

low quality score, our results did not show such a relation between the quality score and SUV $_{\text {mean }}$ of the liver [8].

\section{Study NTR3628: brain imaging}

With respect to dedicated brain imaging, imaging related problems were encountered both during set-up and after central imaging review. Trial set-up was in 2012 and it was planned to have four hospitals involved, but one did not have a MRI scanner that could meet the required technical standards and was excluded from participation. Two other hospitals used MRI protocols that did not meet the required standards $(5 \mathrm{~mm}$ instead of $1 \mathrm{~mm}$ slices, no MTC technique), but they agreed to update their protocols to be able to participate in the study.

Two-hundred-fifteen neurologically asymptomatic, extracranial stage III (based on the ${ }^{18}$ F-FDG-PET-CE-CT) NSCLC patients were included between December 2012 and July 2016. After central review, it turned out that in 56 patients $(26 \%)$ the CE-CT of the brain did not meet the criteria for a dedicated brain imaging protocol. Remarkably, for these patients CE-CT brain results were reported in the radiology report without noting the limitations of the study. Problems with these brain CE-CTs were the field of view ((FOV), adjusted to the thorax, but not to the brain) and the arm position (above the head instead of at thorax level, see Figure 4.1).

\section{Discussion}

Imaging techniques have significantly evolved over the last years and have become more sensitive for the detection of subtle abnormalities. In (lung) cancer guidelines, 


\begin{tabular}{|c|c|c|}
\hline Image Modality & Recommendations & Consequences when not performed \\
\hline MRI brain & $\begin{array}{l}\text { T2 } \\
\text { T2-FLAIR } \\
\text { DWI } \\
\text { T1 before contrast } \\
\text { T1 post contrast } \\
\text { Axial slice thickness between } 3 \mathrm{~mm} \text { and } 5 \mathrm{~mm} \\
\text { Minimal } 1.5 \mathrm{~T}\end{array}$ & $\begin{array}{l}\text { Combination of sequences gives a complete overview, } \\
\text { missing a sequence can lead to a wrong diagnosis (e.g.) } \\
\text { benign vs. malignant) } \\
\text { With thicker slices small lesions can be missed } \\
\text { Too low signal-noise ratio and lower resolution can lead } \\
\text { to missing small lesions }\end{array}$ \\
\hline CE-CT brain & $\begin{array}{l}\text { Field-of-view should be set to head } \\
\text { Arms of the patients at thorax level } \\
\text { Delayed imaging } \\
\text { Higher contrast dose }\end{array}$ & $\begin{array}{l}\text { Artefacts } \\
\text { Artefacts } \\
\text { Less contrast in the head } \\
\text { Lesion less visible }\end{array}$ \\
\hline
\end{tabular}

If any of the recommendations is not met, please consult your nuclear medicine or radiology expert.

Table 4.1: Recommendations and consequences for a quality check of repeated ${ }^{18}$ F-FDG-PET/CT scans, brain CE-CT scans and brain MRI scans based on current guidelines, expert opinion and results of two clinical trials

recommendations regarding imaging techniques are made and even separate detailed ${ }^{18}$ F-FDG-PET-(CT) recommendations exist $[1,2,3]$. However, no recommendations are made on the minimal technical requirements, resulting in a large variation in accuracy. Therefore, there is a large variability in imaging parameters and scanners, which can influence not only the clinical trial outcome but also the clinical interpretation by the treating physician. In this manuscript, we report on two trials in which problems were encountered due to variation in applied imaging protocols, which people did not realize beforehand.

In NTR3628, after central imaging review, it turned out that the CE-CTs brain of several included patients suffered from unreported serious flaws. In this specific trial, the non-diagnostic CE-CT had no clinical consequences, as all patients underwent a postGd-MRI. However, when limitations of an examination are not recognized nor reported, this can have serious consequences (see Table 4.1). Depending on geographical location and institution, in $48-62 \%$ of patients a CE-CT is performed instead of a Gd-MRI to screen for brain metastases [9]. Although not reported in CE-CT imaging guidelines, is the importance of the patient position: it should be reproducible and standardized, and it matters what the FOV and patient position is. The FOV should be adjusted to the size of the head and arms should be at thorax level to reduce artifacts and acquire diagnostic images (see Figure 4.1). Furthermore, just the application of iodine contrast administration does not make the CT-brain diagnostic: timing of the scan relative to contrast injection and the amount of contrast material injected are important.

For tumor response assessment using ${ }^{18} \mathrm{~F}-\mathrm{FDG}-\mathrm{PET}$, it is important that scans are performed with the same acquisition and reconstruction settings as baseline. Because varying uptake times lead to variations in measured SUV, a larger uptake time will result in a higher SUV because tumor SUV increases over time. In response assessment studies, 
changes in SUV over time are the parameter of interest and therefore differences in uptake times between repeated scans can lead to scan misinterpretation. Variability in uptake times of the baseline as well as the response scan made that only 46 scans could be used for early response assessment in the NVALT12 sub-study. A study of Kuhnert et al. [10] showed that for settings like the injected activity and the uptake time the SUV mean of the liver could be used for quality assessment in compliance with the EANM guideline. This means that in the NVALT12 sub-study the large variability in SUV mean of the liver is most probably caused by the differences in uptake time (although a treatment effect cannot be excluded). In the NVALT12 sub-study no relation between deviation in image parameters, reflected in the quality score, and the SUV mean of the liver was reported. The SUV mean of the liver can therefore not exclude all quality issues, although, it could be used as a quick quality control with reference values between 1.3 and 2.5 .

Our recommendations for clinicians regarding repeated ${ }^{18} \mathrm{~F}$-FDG-PET imaging for response assessment are to try to use the same scanning parameters as for the first scan, or check if the same scan parameters are used as in the previous scan. Scans with an uptake time less than 55 minutes or more than 75 minutes cannot reliable be used and checking the SUV $_{\text {mean }}$ of liver can be used as quality assurance. For data analysis it is important that all scan parameters are noted in the DICOM header, but at least injection time, scan time, patient's body weight, radionuclide dose and the SUV conversion tag should be present. An example of a DICOM header with the minimum required tags is shown in Supplementary Material Figure 4.3.

For brain CE-CT combined with the ${ }^{18}$ F-FDG-PET scan, we recommend that the clinician checks the head FOV, the patient arm position and the contrast dose and timing.

For MRI, at least a T2-weighted sequence, a T2-FLAIR and a T1-weighted sequence pre- and post-contrast should be performed. See for an overview of these recommendations Table 4.1. If any of the recommendations cannot be met it is advised to consult an imaging expert.

In addition, radiologists need to be informed about the indication for the requested scan and therefore clinicians must provide all clinical information that is important to determine the optimal imaging protocol to apply. Next to that, for non-imaging clinicians it is important to evaluate whether the applied imaging protocol is indeed adequate for the requested purpose. Our quality recommendations will potentially lead to a more uniform clinical interpretation and could be helpful during multidisciplinary meetings. These recommendations should preferably be tested prospectively.

\section{Funding}

This work was supported by the Dutch Cancer Society (grant UM 2010-4883).

\section{Disclosure}

Author AD is advisory board member of Roche, Eli Lilly, Astra Zeneca, MSD and Pfizer. Author LH is advisory board member of Boehringer-Ingelheim and MSD. All remaining authors have declared no conflicts of interest. 


\section{References}

[1] R. Boellaard et al. "FDG PET and PET/CT: EANM procedure guidelines for tumour PET imaging: version 1.0". In: Eur J Nucl Med Mol Imaging 37.1 (2010). European journal of nuclear medicine and molecular imaging, pp. 181-200. DOI: 10.1007/ s00259-009-1297-4.

[2] P. E. Postmus et al. "Early and locally advanced non-small-cell lung cancer (NSCLC): ESMO Clinical Practice Guidelines for diagnosis, treatment and follow-up". In: Ann Oncol 28 (2017). Annals of oncology : official journal of the European Society for Medical Oncology, pp. iv1-iv21. DOI: 10.1093/annonc/mdx222.

[3] D. S. Ettinger et al. "Non-Small Cell Lung Cancer, Version 5.2017, NCCN Clinical Practice Guidelines in Oncology". In: J Natl Compr Canc Netw 15.4 (2017). Journal of the National Comprehensive Cancer Network: JNCCN, pp. 504-535.

[4] E. E. de Jong et al. "[18F]FDG PET/CT-based response assessment of stage IV non-small cell lung cancer treated with paclitaxel-carboplatin-bevacizumab with or without nitroglycerin patches". In: Eur J Nucl Med Mol Imaging 44.1 (2017). European journal of nuclear medicine and molecular imaging, pp. 8-16. DOI: 10.1007/s00259-016-3498-y.

[5] M. M. Hochstenbag et al. "MR-imaging of the brain of neurologic asymptomatic patients with large cell or adenocarcinoma of the lung. Does it influence prognosis and treatment?" In: Lung Cancer 42.2 (2003). Lung cancer (Amsterdam, Netherlands), pp. 189-93.

[6] J. Schoenmaekers et al. "MA06.05 Screening for Brain Metastases in Patients with Stage III NSCLC, MRI or CT? A Prospective Study". In: Journal of Thoracic Oncology 12.1 (2017), S371-S372. DOI: 10.1016/j.jtho. 2016.11.418. URL: https://doi.org/10.1016/j.jtho.2016.11.418.

[7] The American College of Radiology. ACR-ASNR-SPR Practice parameter for the performance and interpretation of MRI of the brain. 2013. URL: https://www.asnr. org/advocacy/guidelines-standards/.

[8] E. E. C. de Jong et al. "Quality assessment of positron emission tomography scans: recommendations for future multicentre trials". In: Acta Oncol 56.11 (2017). Acta oncologica (Stockholm, Sweden), pp. 1459-1464. DOI: 10.1080/0284186x. 2017. 1346824.

[9] B. J. Hudson, M. B. Crawford, and J. J. Curtin. "Brain imaging in lung cancer patients without symptoms of brain metastases: a national survey of current practice in England". In: Clin Radiol 70.6 (2015). Clinical radiology, pp. 610-3. DOI: 10.1016/ j.crad.2015.02.007.

[10] G. Kuhnert et al. "Impact of PET/CT image reconstruction methods and liver uptake normalization strategies on quantitative image analysis". In: Eur J Nucl Med Mol Imaging 43.2 (2016). European journal of nuclear medicine and molecular imaging, pp. 249-58. DOI: $10.1007 / \mathrm{s} 00259-015-3165-8$. 


\section{Supplementary Material}

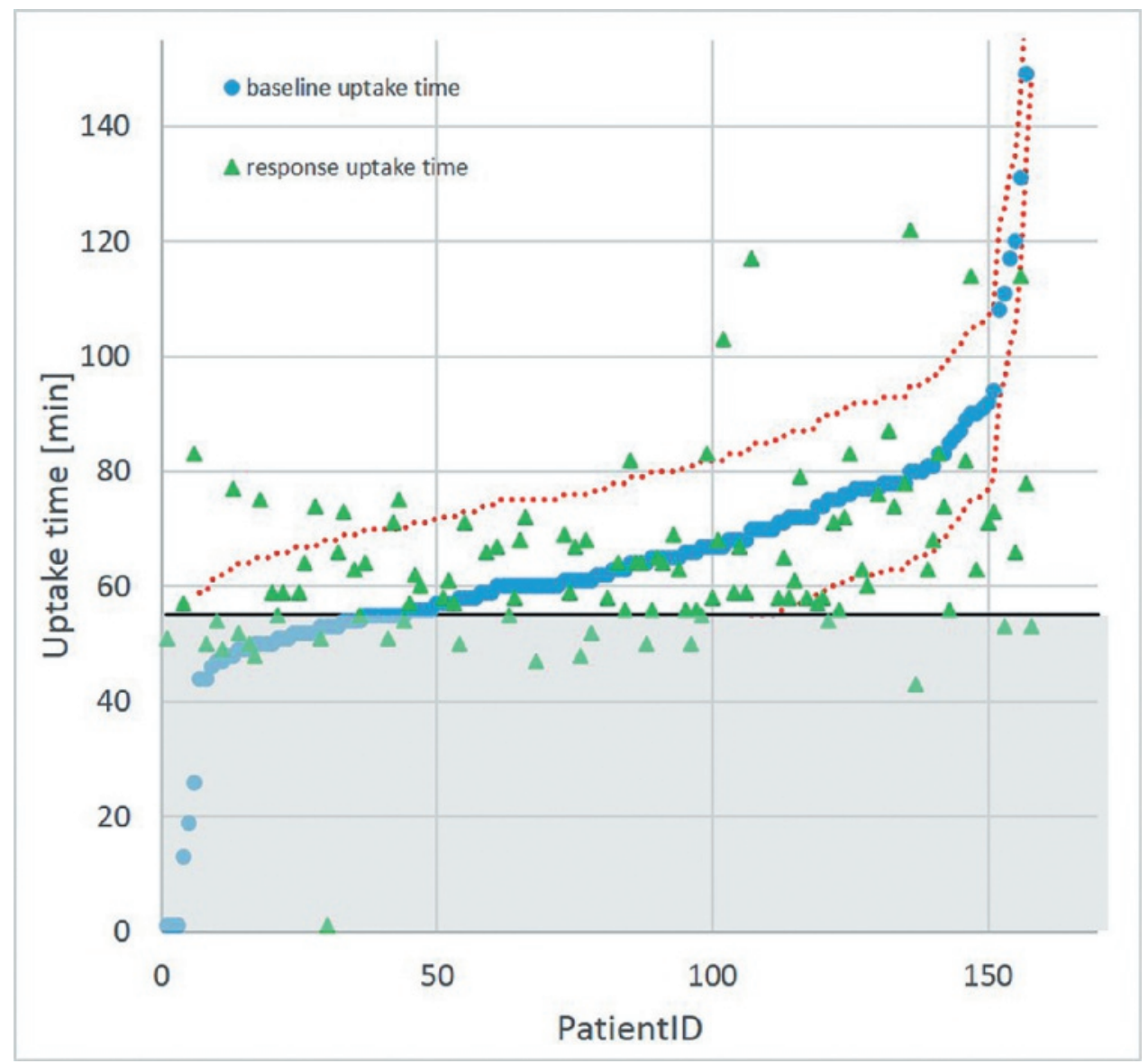

Figure 4.2: Time interval between FDG injection and start of PET scan ranked in ascending order for the baseline scan. The grey area shows uptake times lower than 55 minutes, the red lines show the 15 minutes difference between the repeated scans (i.e. only the scans between the red lines can be used for response assessment). Figure adjusted from [1] 


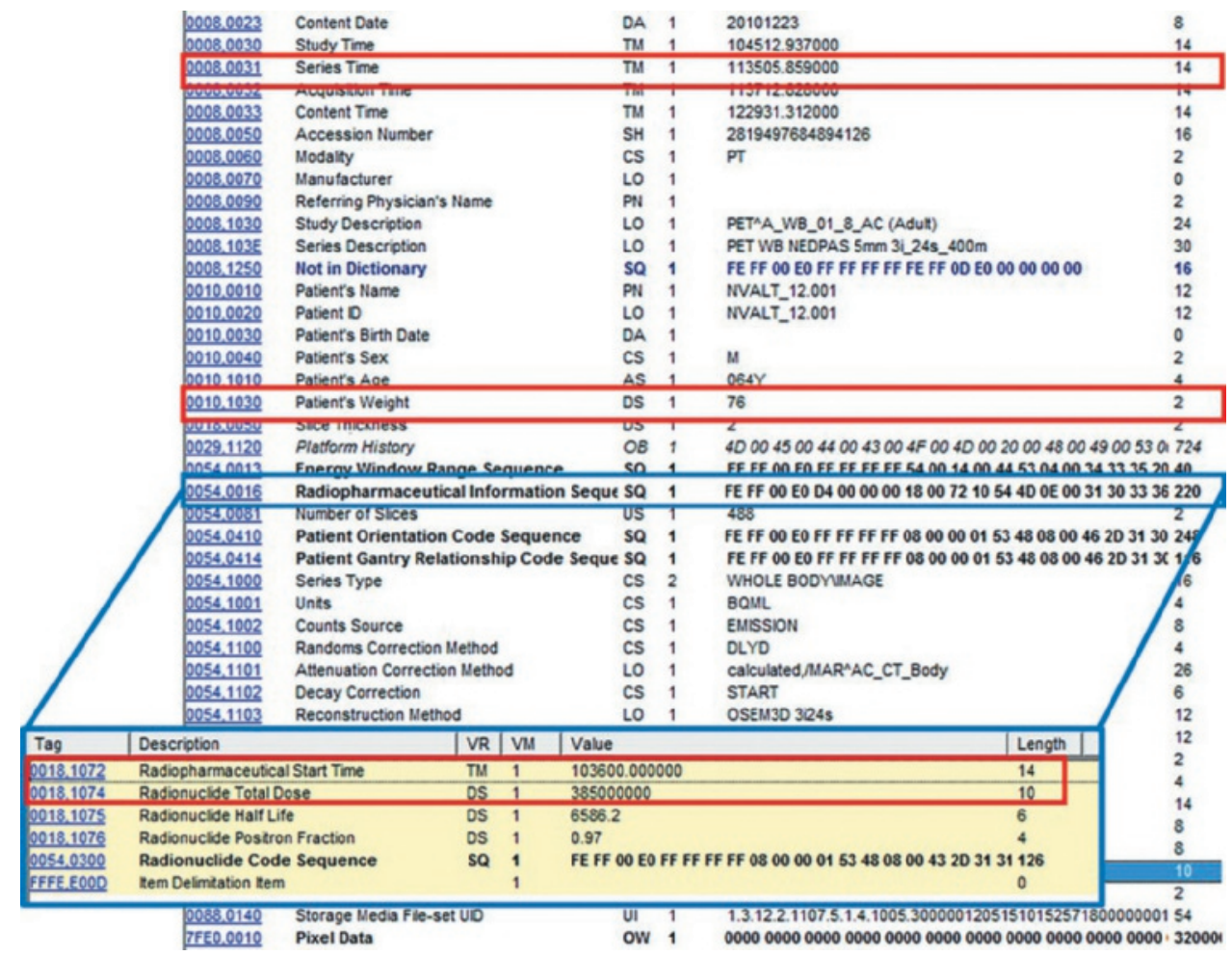

Figure 4.3: Example of a section of a DICOM header from an ${ }^{18}$ F-FDG-PET scan. The red boxes show the minimum required tags. 



\section{Chapter 5}

Influence of gray level discretization on radiomic feature stability for different CT scanners, tube currents and slice thicknesses: a comprehensive phantom study

\section{Authors}

Ruben T.H.M. Larue*, Janna E. van Timmeren*, Evelyn E.C. de Jong*, Giacomo Feliciani, Ralph T.H. Leijenaar, Wendy M.J. Schreurs, Meindert N. Sosef, Frank H.P.J. Raat, Frans H.R. van der Zande, Marco Das, Wouter van Elmpt, Philippe Lambin

*equal contribution 


\section{Abstract}

\section{Background}

Radiomic analyses of $\mathrm{CT}$ images provide prognostic information that can potentially be used for personalized treatment. However, heterogeneity of acquisition- and reconstruction protocols influences robustness of radiomic analyses. The aim of this study was to investigate the influence of different CT-scanners, slice thicknesses, exposures and gray-level discretization on radiomic feature values and their stability.

\section{Material and methods}

A texture phantom with ten different inserts was scanned on nine different CT-scanners with varying tube currents. Scans were reconstructed with 1.5 $\mathrm{mm}$ or $3 \mathrm{~mm}$ slice thickness. Image pre-processing comprised gray-level discretization in ten different bin widths ranging from 5 to $50 \mathrm{HU}$ and different resampling methods (i.e., linear, cubic and nearest neighbor interpolation to $1 \times 1 \times 3 \mathrm{~mm}^{3}$ voxels) were investigated. Subsequently, 114 textural radiomic features were extracted from a $2.1 \mathrm{~cm}^{3}$ sphere in the center of each insert. The influence of slice thickness, exposure and bin width on feature values was investigated. Feature stability was assessed by calculating the concordance correlation coefficient (CCC) in a test-retest setting and for different combinations of scanners, tube currents and slice thicknesses.

\section{Results}

Bin width influenced feature values, but this only had a marginal effect on the total number of stable features (CCC $>0.85$ ) when comparing different scanners, slice thicknesses or exposures. Most radiomic features were affected by slice thickness, but this effect could be reduced by resampling the CTimages before feature extraction. Statistics feature 'energy' was the most dependent on slice thickness. No clear correlation between feature values and exposures was observed.

\section{Conclusions}

CT-scanner, slice thickness and bin width affected radiomic feature values, whereas no effect of exposure was observed. Optimization of gray-level discretization to potentially improve prognostic value can be performed without compromising feature stability. Resampling images prior to feature extraction decreases the variability of radiomic features. 


\section{Introduction}

Extraction of quantitative imaging features, also called radiomics [1], has become an additional source of information for the development of prognostic and predictive models $[2,3,4,5,6,7]$. The total number of features that can be calculated is almost unlimited, especially if filter-based features (e.g., Laplacian of Gaussian or wavelet) are also taken into consideration. To incorporate such large numbers of features in prognostic and predictive models, multiple independent and multi-centric datasets are needed for training and validation. However, recent literature shows that there are some challenges to overcome [8].

Several studies already showed that radiomic feature values are influenced by image acquisition and reconstruction settings, like slice thickness and exposure $[2,9,10,11$, 12, 13]. For instance, Mackin et al. [13] scanned a phantom with ten unique inserts using different acquisition parameters on computed tomography (CT) scanners of four manufacturers. They demonstrated that the variability in textural features calculated on the phantom scans can be in the same order of magnitude as the variability seen in non-small cell lung cancer patients (NSCLC). Using the same phantom, Shafiq-ul-Hassan et al. [12] investigated voxel-size dependency of radiomic features and found that this dependency can be minimized by resampling to a nominal voxel size or by normalizing the voxel size. Next to that, they found that gray-level dependency also can be reduced by normalization. Since multi-centric data usually are acquired with different CT-scanners using institutional scan protocols, the lack of statistical power in validation datasets might, therefore, be attributed to the different acquisition settings.

In the current study, we investigated the variability in radiomic feature values for scans with different slice thicknesses, different exposures and from different CT-scanners and performed a test-retest analysis using the same texture phantom as Mackin et al. and Shafiq-ul-Hassan et al. [13, 12]. In addition to these former studies, we used a more extensive radiomic features dataset, different scanner types and focussed more on the influence of gray-level discretization and resampling of voxel size on interchangeability of radiomic features. Furthermore, we compared the variability of radiomic features values derived from the phantom with those derived from two independent non-small cell lung cancer (NSCLC) patient datasets, to investigate the comparability of the phantom inserts to clinical CT-scans.

\section{Material and Methods}

\section{Image acquisition}

The Credence Cartridge Radiomics phantom (CCR), previously described by Mackin et al. [13], consists of an acrylic case with ten different inserts with different textures and was scanned on nine different CT-scanners. We focussed on the shredded rubber insert, because the CT-properties of this insert are most similar to tumors [13].

An overview of all scanners and settings is provided in Table 5.1. The scans were reconstructed using two different slice thicknesses per scanner. For the ' $\mathrm{H}$ ' scanner, the ' $\mathrm{B}$ ' scanner and the ' $\mathrm{O}$ ' scanner the increment as well as the slice thickness was $1.5 \mathrm{~mm}$ or $3 \mathrm{~mm}$. For the other scanners the increment was $1 \mathrm{~mm}$ for scans with a slice thickness of $1.5 \mathrm{~mm}$ (scanner ' $\mathrm{S}$ ' had a slice thickness of $2 \mathrm{~mm}$ instead of 1.5 ) and $2 \mathrm{~mm}$ for the 
scans with a slice thickness of $3 \mathrm{~mm}$. The reconstructed field of view was $500 \mathrm{~mm}$ for all scans.

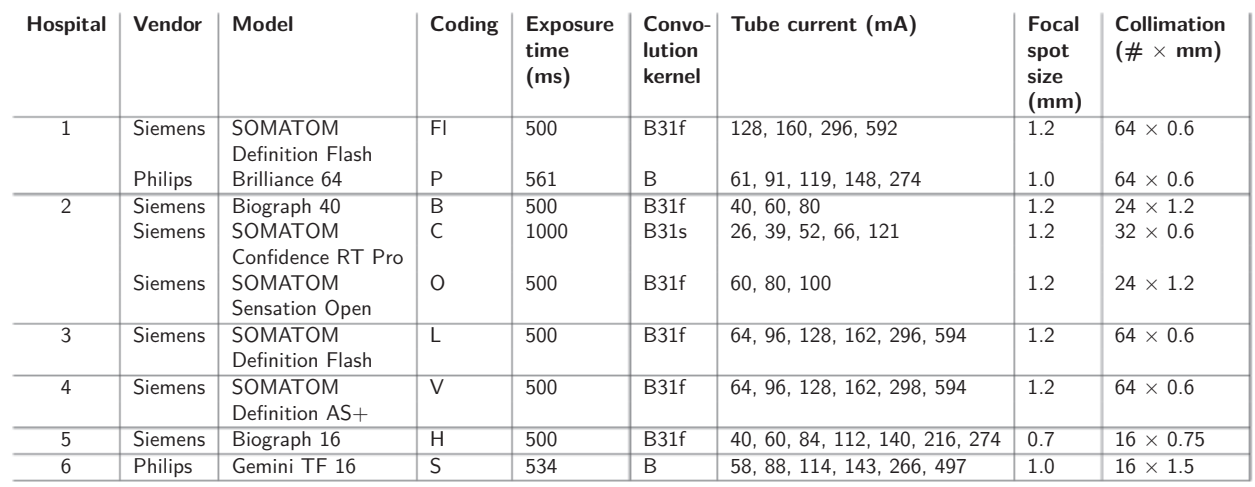

Table 5.1: Overview scanners and per scanner exposure time, convolution kernel, tube current, focal spot size and collimation.

All scans were performed with a tube voltage of $120 \mathrm{kVp}$ and a pitch of 1.0 . The aim was to scan the phantom with the same range of CT-dose index (CTDI) settings [2.17, $3.26,4.32,5,43,10,20 \mathrm{mGy}$ ], but due to hardware (e.g., the risk of overheating) and software limitations (i.e., not all exposures could be set) not all CTDls could be obtained for all scanners. Philips scans were reconstructed using the B kernel and Siemens scans using the B31f or B31s kernel.

For test-retest purposes, two subsequent CT-scans were acquired on the 'B' and the ' $\mathrm{O}$ ' scanner. The phantom was kept in place on the table without changing anything in between, meaning that no other parameters than scanner output fluctuations could have influenced the images.

\section{Feature extraction}

A spherical region of interest (ROI) with a volume of $2.1 \mathrm{~cm}^{3}$ was contoured using Mirada RTx (Mirada RTx 1.6, Mirada Medical, Oxford, UK) in the center of every insert. Moreover, for test-retest purposes, the ROI was shifted to the right and downwards. Supplementary Material Figure 5.7 shows an example CT image of the phantom in which the ROls are indicated in the rubber insert.

One hundred and fourteen radiomic features were extracted for the ROI in every insert using software developed in-house. The histogram of voxel intensity values within the ROI was described by nineteen first order statistics (Stats) features. Textural features were divided in five neighborhood gray-tone difference (NGTDM) features, sixteen neighboring gray-level dependence matrix (NGLDM) features, sixteen gray-level size zone matrix (GLSZM) features, sixteen gray-level run length matrix (GLRLM) features, sixteen gray-level distance zone matrix (GLDZM) features and 26 gray-level co-occurrence matrix (GLCM) features. The definitions of the radiomic features are previously described in van Timmeren et al. [14].

The following pre-processing steps were applied prior to radiomic feature extracting: gray-level discretization and voxel resampling. All features were calculated for ten bin widths ranging from 5 to 50 Hounsfield Units (HU), with a step size of 5 HU. Radiomic 
features were also calculated with and without applying resampling into voxel sizes of $1 \times 1 \times 3 \mathrm{~mm}^{3}$ using cubic, linear and nearest neighbor interpolation, which was only done for a bin width of 25 .

\section{Analyses}

The variation in feature values due to slice thickness and exposure, calculated per feature for the rubber insert using a bin width of $25 \mathrm{HU}$, was defined as the maximal difference between scans with either the same exposure (variation due to unequal slice thicknesses) or the same slice thickness (variation due to unequal exposures). The ratios between the variations were calculated to investigate which features are most dependent on either slice thickness or exposure. Features were ranked per scanner based on their ratios and afterwards the rankings were summed, with a higher total rank indicating larger dependency on slice thickness, and a lower total rank indicating a larger dependency on exposure.

We compared the radiomic feature values which were calculated using the three different resampling methods. Moreover, for five different scanners we compared the distribution of $\mathrm{HU}$ values in the rubber insert. For all scans we used in this analysis, the phantom was scanned using a CTDI of $5.43 \mathrm{mGy}$ and reconstructed with a slice thickness of $1.5 \mathrm{~mm}$. We also investigated the $\mathrm{HU}$ distributions after resampling into voxel sizes of $1 \times 1 \times 3 \mathrm{~mm}^{3}$ using linear interpolation.

To investigate the influence of gray-level discretization on the feature values, each feature was plotted against the bin width. Moreover, the pairwise concordance correlation coefficient (CCC) [15] was used to determine the agreement in feature values over all inserts when comparing (1) a slice thickness of $1.5 \mathrm{~mm}$ with $3 \mathrm{~mm},(2)$ an exposure of 60 $\mathrm{mA}$ with $80 \mathrm{~mA}$, or (3) scanner ' $\mathrm{B}$ ' with 'O' using the same scan protocol. The CCC ranges from -1 (perfect negative agreement) to 1 (perfect positive agreement). A minimum CCC of 0.85 , which was used in previous studies $[16,17]$, was used to identify features that were independent of the different scanners or settings that were compared. The total number of stable features per bin width was then used as a measure to investigate the influence of bin width on feature stability.

Moreover, we performed a test-retest analysis to be able to compare results within a controlled setting. For the three sets of scans that were used to perform the CCC analyses described above: 1) B-60mA-3mm and B-60mA-1.5mm, 2) B-60mA-3mm and B-80mA-3mm and 3) B-60mA-3mm and O-60mA-3mm, we also calculated the CCC for the test-retest setting of both scans. The minimal CCC of these two test-retest scans was then used to determine the number of stable features $(C C C>0.85)$ per bin width. Moreover, we investigated the variability of radiomic features caused by shifted ROls (see 'Feature extraction'), by calculating the CCC between the radiomic features extracted from the scan with the ROI in the center and from the same scan with the shifted ROI.

Finally, to test if the variability in tumor feature values is comparable to the variability in feature values measured in the phantom, the results from the ten phantom inserts were compared to two patient datasets. Patient dataset 1 consisted of 157 NSCLC patients for which the CT-scans were acquired in different hospitals in which the phantom was scanned as well. Patient dataset 2 consisted of 168 NSCLC patients for which all CT-scans were performed in a single hospital which is one of the hospitals in which the phantom was scanned. All patient scans had a slice thickness ranging from $1 \mathrm{~mm}$ to $3 \mathrm{~mm}$. To reduce variability between scans, these were resampled to voxels of $1 \times 1 \times 3 \mathrm{~mm}^{3}$ prior to analysis using cubic interpolation, as this corresponds to the voxel size of most the clinical images. 


\section{Results}

The feature 'First order statistics (Stats) - Energy' had the highest rank and thus showed the highest dependency on slice thickness and 'GLRLM - Run Length Nonuniformity (RLN)' the second highest dependency (Supplementary Material Figure 5.8). The feature 'GLSZM - Small Area Emphasis (SAE)' was least dependent on slice thickness and was ranked last. In further analyses in this study, we focussed on the features 'GLRLM RLN' and 'GLSZM - SAE', since 'Energy' is independent of gray-level discretization by definition. Figure 5.1 shows that the feature values for 'GLRLM - RLN' for $1.5 \mathrm{~mm}$ and $3 \mathrm{~mm}$ were more similar after resampling, which was not the case for the 'GLSZM SAE' feature values. The test-retest analysis demonstrated that the feature 'GLRLM RLN' is a stable feature (CCC $>0.85$ for all bin widths), whereas the feature 'GLSZM SAE' is unstable (Supplementary Material Figure 5.10).
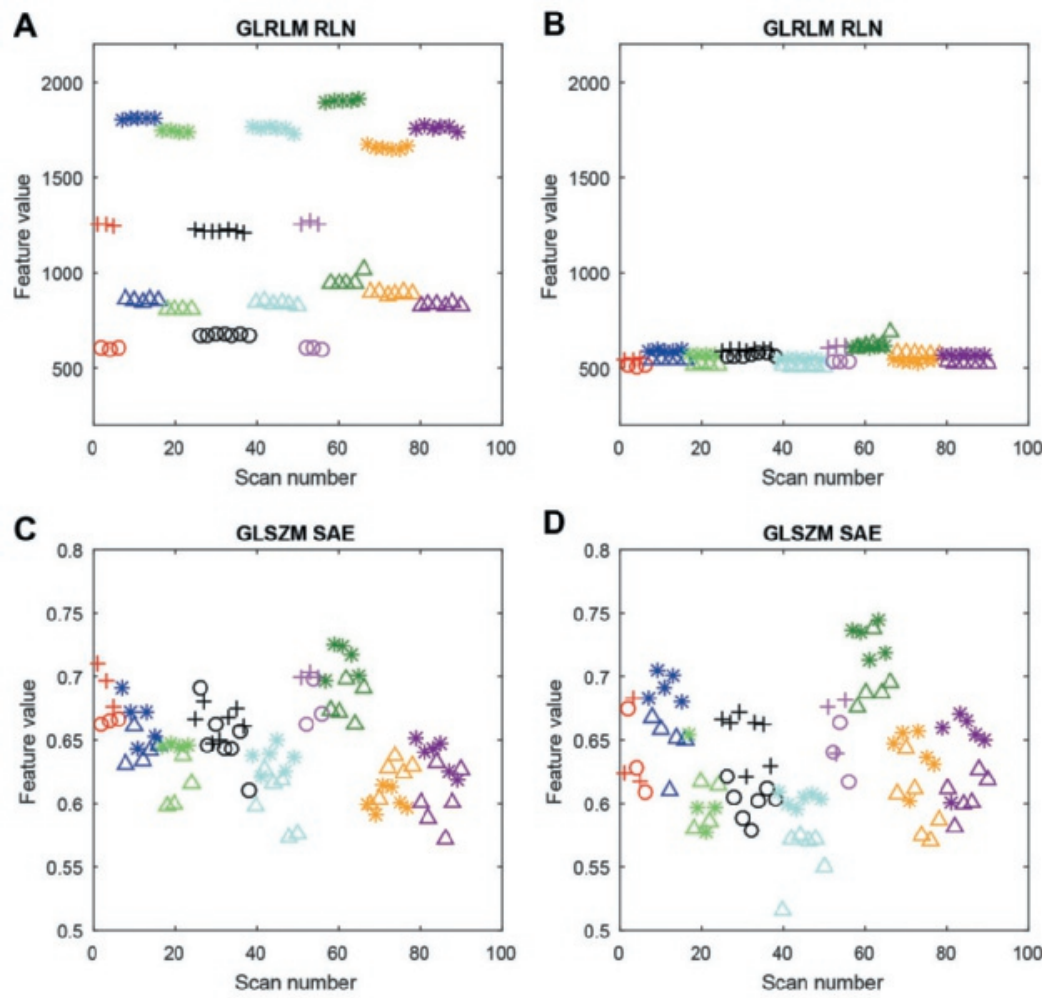

\begin{tabular}{llll}
\hline Siemens Biograph 40 & - Siemens Sensation Open & $* 1 \mathrm{~mm}$ \\
- Siemens Confidence RT Pro & - Philips Brilliance 64 & $\Delta 2 \mathrm{~mm}$ \\
- Siemens Definition Flash & - Philips Gemini TF 16 & $+1.5 \mathrm{~mm}$ \\
- Siemens Biograph 16 & - Siemens Definition AS+ & 0 & $\mathrm{~mm}$ \\
- Siemens Definition Flash & & &
\end{tabular}

Figure 5.1: Scatterplots of feature values for all scanners. Per scanner, the data is sorted from high to low exposure. Left: original data; Right: after resampling; Top: 'GLRLM-RLN', highest slice thickness dependency; Bottom: 'GLSZM-SAE', lowest slice thickness dependency. 
For the feature 'GLRLM - RLN' the cubic, linear and nearest neighbor resampling method resulted in very similar feature ranges after resampling (Figure 5.2). For 'GLSZM - SAE', however, the nearest neighbor method resulted in a very wide range. When comparing the three resampling methods for all features tested $(n=114)$, for $34(30 \%)$ features cubic interpolation resulted in the narrowest feature range, for 55 (48\%) features this applied to linear interpolation, and the remaining 25 (22\%) features had the narrowest feature range after nearest neighbor interpolation. Nearest neighborhood interpolation had the widest range for the majority $(61 \%)$ of the features. For the feature that showed the highest dependency on slice thickness, 'Stats - Energy', feature values over all scans ranged from $6.3 \times 10^{8}$ to $1.9 \times 10^{9}$ (median $1.2 \times 10^{9}$ ), which reduced to a range from $5.5 \times 10^{8}$ to $6.9 \times 10^{8}$ (median $6.1 \times 10^{8}$ ) after resampling using cubic interpolation.

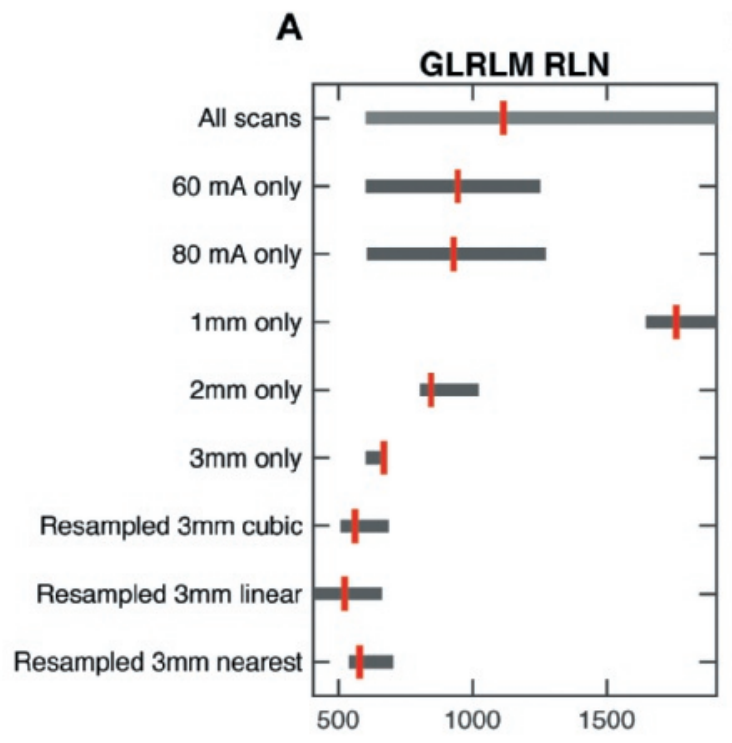

\section{B}

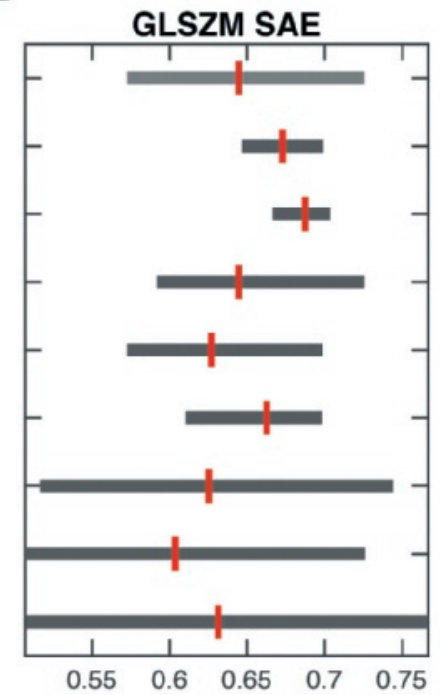

Figure 5.2: Bar plots of the spread of feature values of (A): 'GLRLM-RLN' and (B): 'GLSZM-SAE'. Bars range from the minimum to the maximum observed value, and the vertical lines indicate the median. From top to bottom these bars are shown for all scans $(n=90)$, all scans with an exposure of $60 \mathrm{~mA}(n=6)$, all scans with an exposure of $80 \mathrm{~mA}(n=4)$, all scans with an increment of 1 $\mathrm{mm}(\mathrm{n}=34), 2 \mathrm{~mm}(\mathrm{n}=34)$ and $3 \mathrm{~mm}(\mathrm{n}=13)$ and all scans after resampling the images into $1 \times 1 \times 3 \mathrm{~mm}^{3}$ using cubic $(n=90)$, linear $(n=90)$ and nearest neighbor $(n=90)$ interpolation.

Figure 5.3 shows that the scans of the five different scanners had a similar median HU value: between 931 and $939 \mathrm{HU}$. When comparing the range in $\mathrm{HU}$ of those five scanners, scanner ' $\mathrm{P}$ ' had a much wider range than the other four scanners (160-1564 compared to 437-1291). As shown in Figure 5.3 for the feature 'GLCM cluster prominence', this also affected radiomic feature values extracted from these images. The same histograms were made after resampling, which show a similar shape as before resampling (shown in Supplementary Material Figure 5.9). 

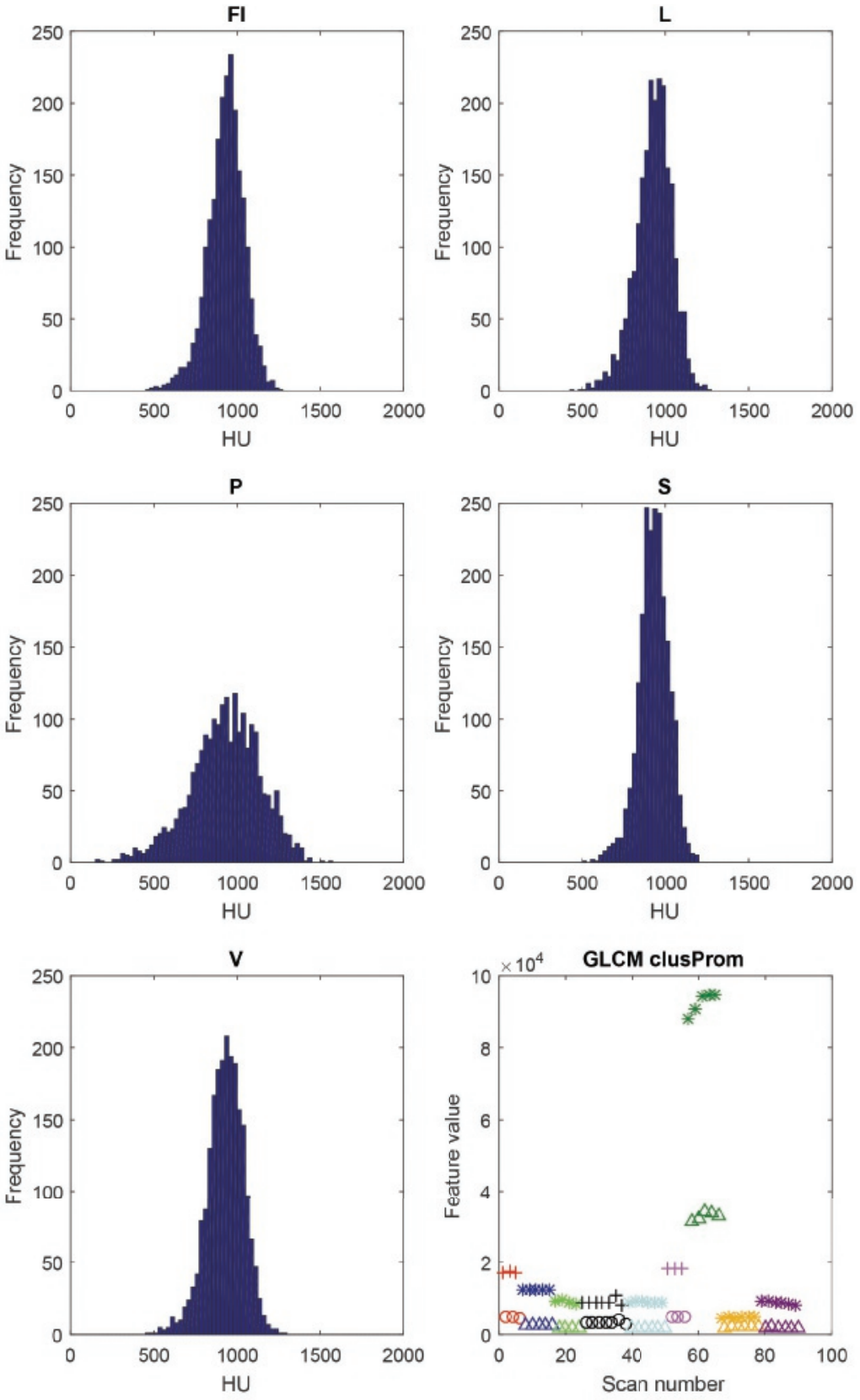

Figure 5.3: HU distribution of the rubber insert scanned with a fixed CDTI of $5.43 \mathrm{mGy}$ on five different Siemens and Philips scanners. From left to right and top to bottom [scanner tube current]: 'Fl-160mA', 'L-161mA', 'P-148mA', 'S-143mA', and 'V-162mA'. The scatterplot in the bottom right displays the observed values of the feature 'GLCM cluster prominence'over all scanners. The outlier in dark-green is scanner ' $P$ '. 

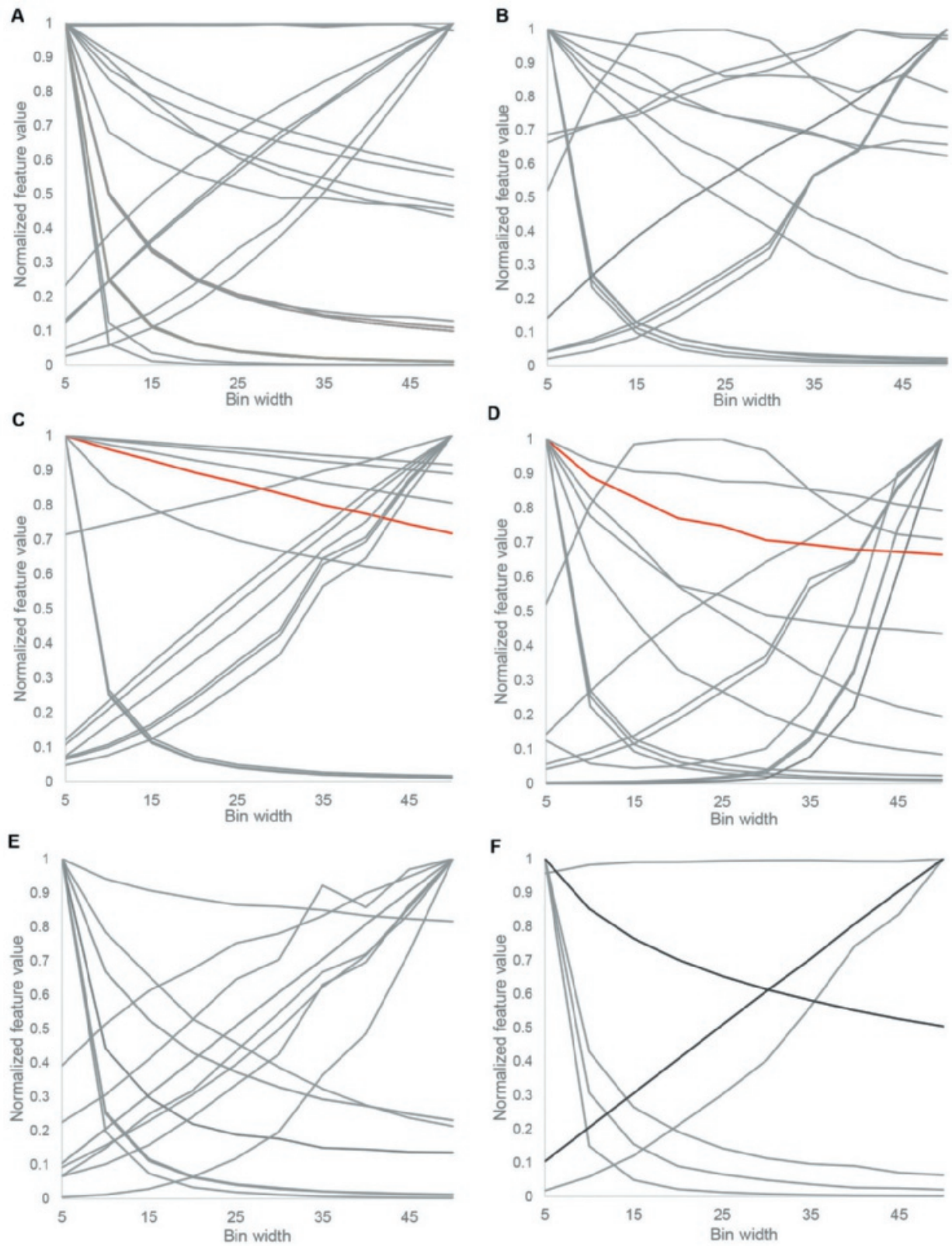

Figure 5.4: Normalized feature values plotted against bin width. (A): GLCM features $(n=26)$, (B): GLDZM features $(n=16),(C)$ : GLRLM features $(n=16),(D)$ : GLSZM features $(n=16),(E)$ : NGDLM features $(n=16),(F)$ : NGTDM $(n=5)$ and first order statistics features $(n=2$, dotted lines). The red dashed lines indicate GLRLM RLN and GLSZM SAE. 
Figure 5.4 shows that every normalized feature had another bin width dependency. Some features (e.g., 'Energy' and 'Skewness') were not dependent on bin width per definition. In terms of feature stability, when comparing $1.5 \mathrm{~mm}$ scans with $3 \mathrm{~mm}$ scans using an exposure of $60 \mathrm{~mA}$, the total number of stable textural features hardly changed over the different bin widths (Figure 5.5). The median number of stable features was 49 (range 47-53). All CCC numbers for this comparison are shown in Supplementary Material Figure 5.10, where the features that were not stable in either the test-retest of 'B-60mA-1.5mm' or 'B-60mA-3mm' are also indicated. Also, when comparing an exposure of $60 \mathrm{~mA}$ with $80 \mathrm{~mA}$ on the same scanner, or scanner 'B' with 'O', no trend indicating a dependency on bin width could be observed. In this case, the median number of stable features was 66.5 (55-70) and 67.5 (56-72), respectively. Note that the total number of stable features was much lower when comparing different slice thicknesses.

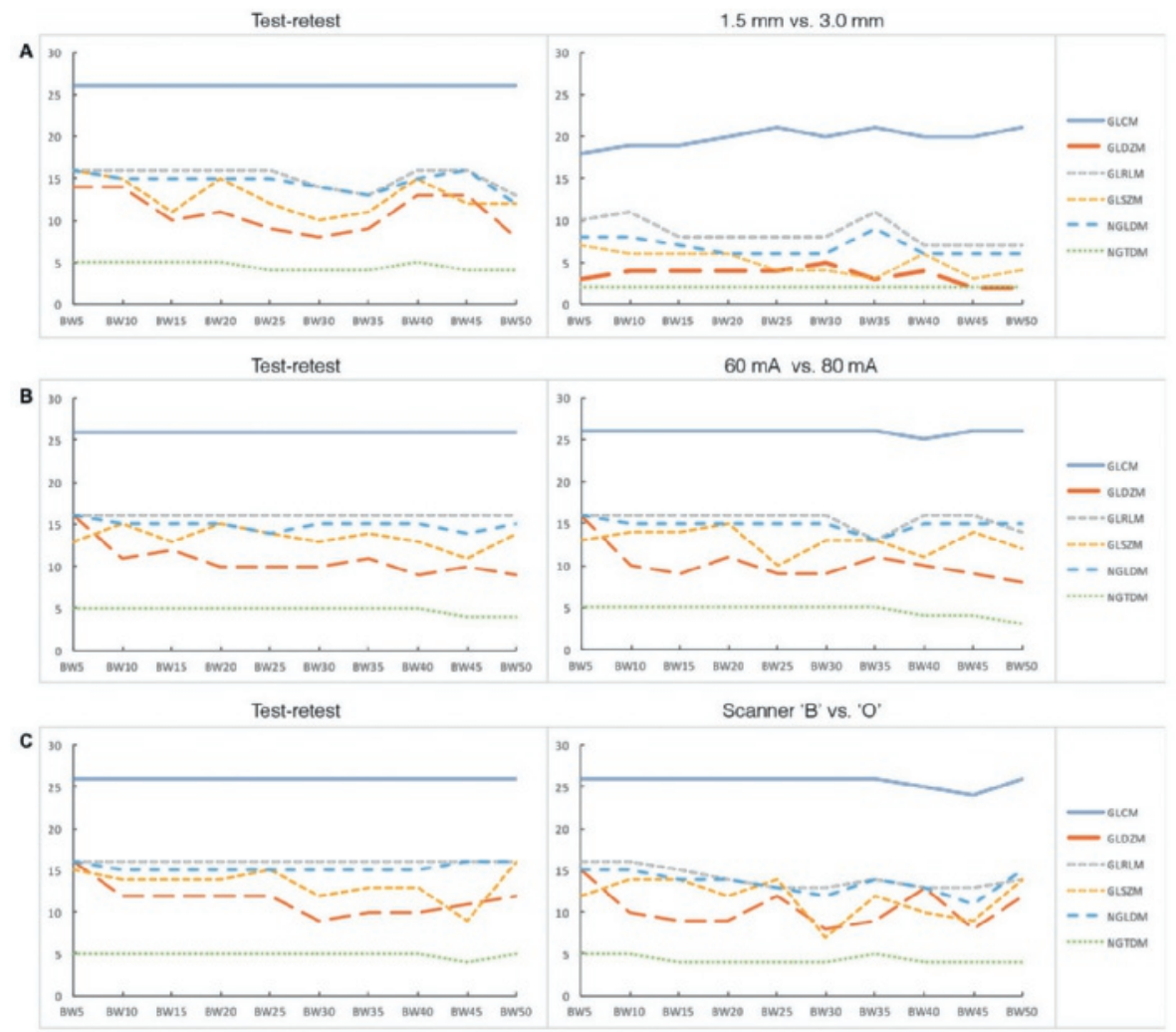

Figure 5.5: The total number of features with a CCC $>0.85$ for each bin width when comparing (A) a slice thickness of $1.5 \mathrm{~mm}$ with $3 \mathrm{~mm}$ (exposure $60 \mathrm{~mA}$, scanner 'B'), (B) an exposure of $60 \mathrm{~mA}$ with $80 \mathrm{~mA}$ (slice thickness $3 \mathrm{~mm}$, scanner 'B'), or (C) scanner 'B' and 'O' using the same scan protocol (exposure $60 \mathrm{~mA}$ and slice thickness $3 \mathrm{~mm}$ ). Test-retest figures are based on minimal observed CCC in both test-retest sets that are compared. 


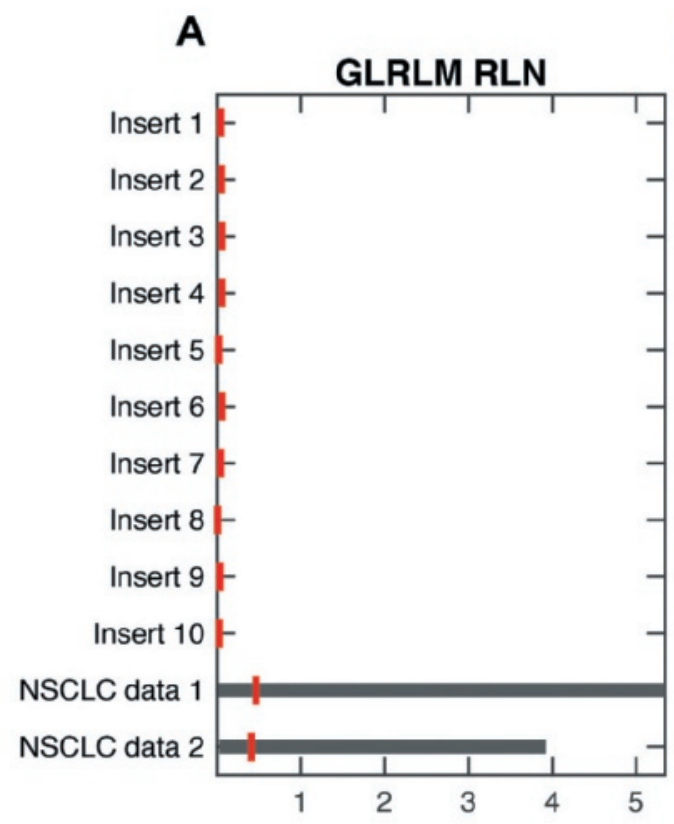

B

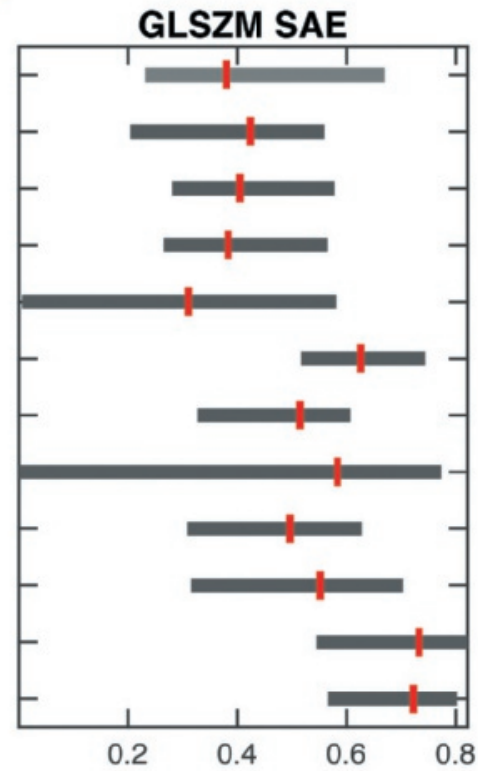

Figure 5.6: Bar plots of the spread of feature values over all phantom inserts and two independent NSCLC datasets for (A): 'GLRLM-RLN' and (B): 'GLSZM-SAE'. The bars indicate the minimum, median, and maximum feature values. Scans were resampled to $1 \times 1 \times 3 \mathrm{~mm}^{3}$ voxels using cubic interpolation and a bin width of $25 \mathrm{HU}$ was used.

The images on the left side of Figure 5.5 show that approximately the same number of features were stable for the test-retest compared to the scans with different exposure or acquired from different scanners. On the other side, more features were stable in the testretest setting than when slice thicknesses were different. The test-retest analysis of scan 'B-60mA-3mm' (bin width 25) shows that 5 out of 114 had a CCC below 0.85 . When shifting the ROI downwards (ROI '6s324' in Supplementary Material Figure 5.7), there were 8 features with CCC $<0.85$, with $100 \%$ overlap compared to the test-retest. When shifting the delineation to the right (ROI '6s234'), 14 features were unstable, again with a $100 \%$ overlap.

The range of feature values for all inserts was compared to the range in two independent datasets of NCSLC patients. As shown in Figure 5.6, the 'GLRLM-RLN' feature range observed in the phantom is very limited and substantially lower in comparison with the range observed in the clinical datasets, whereas the 'GLSZM-SAE' ranged in the same order of magnitude. The same plots for all features are available in Supplementary Material Figure 5.11. 


\section{Discussion}

The aim of this study was to investigate the impact of different CT-scanners, slice thicknesses, exposures, gray-level discretization and resampling of voxel sizes on feature values and on their stability using a texture phantom. Moreover, we performed a test-retest analysis. In short, we could show that CT-scanner, slice thickness and gray-level discretization (i.e. bin width) influence feature values. A clear effect of exposure on feature values was not observed. Moreover, the stability of radiomic features is hardly influenced by bin width, i.e. for each bin width the variability across different settings is similar.

Scatterplots of feature values showed that the distribution of feature values is different for each CT-scanner. Since scans were acquired with the same range of CTDI values, this implies that the variability of feature values is affected by different scanners used in that particular study. The feature 'GLSZM - SAE', which has the lowest rank in terms of slice thickness dependency, appeared to be unstable in a test-retest analysis. In contrast the feature 'GLRLM - RLN' had a CCC above 0.85 for all test-retest scans, whereas the CCC was low in the comparison between $1.5 \mathrm{~mm}$ and $3 \mathrm{~mm}$ slice thickness (Supplementary Material Figure 5.10).

A comparable effect was observed in the HU histograms of five different scans that were acquired with the same CTDI of $5.43 \mathrm{mGy}$. The ' $\mathrm{Fl}$ ', 'L', ' $\mathrm{V}$ ', and 'S' scanners had comparable $\mathrm{HU}$ distributions within the rubber insert. However, even though the same radiation dose output of the CT-scanner and the same acquisition protocol was used, scanner ' $\mathrm{P}$ ' resulted in a CT-image with a much wider range of HU. The histograms show the same pattern after resampling of the images was applied. The reason for the discrepancy of scanner ' $P$ ' is unknown, but it might be the result of an incorrect calibration of the scanner. So even with similar acquisition protocols, different scanner types within one dataset can influence radiomic feature values. Therefore, we recommend to take this into account when performing radiomic studies with multiple heterogeneous datasets, i.e., to perform a study specific test-retest analysis [16] to eliminate features that are not robust across different scanners.

We also showed that a large proportion of features is influenced by the slice thickness used for reconstruction. However, we could show that the variability in feature values decreased after resampling was performed. This is in line with Shafiq-ul-Hassan et al. [12], who showed that resampling reduced the feature variability from $\%$ COV $>70 \%$ to $\% \mathrm{COV}<30 \%$. Therefore, we recommend to always perform resampling prior to any radiomic analysis. Despite the fact that resampling can greatly improve the robustness of radiomic features [12], we recommend to keep the voxel size as consistent as possible, since the variability in feature values is even lower when the voxel size is equal for all images included in the study. This should also be taken into account when comparing training and validation datasets that might be reconstructed into different voxel sizes, which could have increased the discrepancy between datasets in terms of distribution of radiomic feature values although resampling was applied. In our study the data was resampled to a voxel size of $1 \times 1 \times 3 \mathrm{~mm}^{3}$ using a cubic, linear and nearest neighbor interpolation, while in the study of Shafiq-ul-Hassan et al. [12] the data was resampled to a voxel size of $1 \times 1 \times 2 \mathrm{~mm}^{3}$ using a linear interpolation. Our results demonstrated that linear interpolation resulted in the narrowest feature value range for $48 \%$ of the features and cubic interpolation for $30 \%$ of the features, whereas nearest neighbor interpolation had the widest range for $61 \%$ of the features. Therefore, cubic or linear interpolation are preferred over nearest neighbor 
interpolation when resampling to $1 \times 1 \times 3 \mathrm{~mm}^{3}$ voxels.

Furthermore, we investigated the influence of exposure on the feature values, as tube current modulated CT-scans become more common. We only had data from a few scans per CT-scanner, which made it difficult to investigate the potential influence of exposure on radiomic feature values. For none of the features, we could see a clear relationship between feature value and the exposure. Mackin et al. [13] had quite a large range of exposure values for the different scanners, although the effect of exposure on feature values was not investigated, this might have influenced the scanner comparison results. In the future, the influence of exposure should be explored on a larger dataset.

Finally, we investigated the influence of the pre-processing step of 'gray-level discretization' on feature values and on their stability [18]. The main goal was to find an optimal bin width that could result in the highest reproducibility of radiomic features. Almost all features change in value when choosing another bin width for gray-level discretization. For a subset of features, a very small or very large bin width resulted in very different feature values across scans, whereas feature values are more similar with a bin size in the order of 25 (Figure 5.4). Although the feature values change for different bin widths, we were not able to show that the stability of radiomic features is greatly influenced by the choice of bin width (Figure 5.5 and Supplementary Material Figure 5.10). Our finding is the contrary of what Shafiq-ul-Hassan et al. [12] described. They found that only seven out of 51 features were reproducible independent of the gray-level discretization. Next to that, they found that seventeen out of 44 features showed a trend with varying number of gray-levels, which could be a linear, quadratic or cubic-type relation. Normalization of feature values by the number of gray-levels reduced the variation in feature values. However, these results imply that the choice of bin width could alter the prognostic value of a certain radiomic signature. The influence of gray-level discretization on the prognostic value of radiomic features has not yet been investigated and needs further research. Therefore, we cannot indicate a certain bin width as being most optimal, but we strongly recommend to be consistent and always clearly report which pre-processing steps have been used to improve the reproducibility and validation of radiomic studies [9].

The test-retest analysis we have performed shows that, even when not changing anything in between two subsequently acquired CT scans, some radiomic features were not robust. When shifting the ROI within the same insert, even more features failed to reach a CCC above 0.85 . The only reason for the instability of features in the test-retest setting could be the variability of scanner output, which is always present and cannot be avoided. Therefore, these features would be too unstable even when imaging protocols are completely standardized. We acknowledge that it is difficult to define a CCC threshold for eliminating the features, but we think that it is reasonable to exclude the non-stable radiomic features with CCC $<0.85$ in future radiomic studies.

One of the limitations of this study is the ignorance of convolution kernel used during reconstruction. We considered the Philips 'B' kernel and the Siemens 'B31s' and 'B31f' as interchangeable, but this was not investigated. We also did not investigate the influence of iterative kernels or others commonly used in clinical practice, which might also affect the variability of radiomic features.

In this study, we investigated the influence of factors which we expected to have a major effect on radiomics. Nonetheless, other parameters influence image quality and therefore could have influenced the results. For example, scanners from different manufacturers have different possibilities for focal spot size and collimation width due to differences in technical 
design. These parameters do influence the image appearance: a smaller collimation width results in increased noise and a larger focal spot size results in decreased image quality (i.e. reduced sharpness). In the original design of the study we did not control for these parameters and the values were only traced back after the study was performed. In future studies, the influence of these parameters on radiomic features should be evaluated, and we recommend to report as much parameters as possible.

Furthermore, the potential influence of change in volume of the spherical region of interest was not investigated. Although all images were registered to the same reference scan which was used for delineations, this always leads to slightly different volumes. Whereas the spherical volume should consistently be $2.1 \mathrm{~cm}^{3}$, we noticed deviations in the order of $0.1 \mathrm{~cm}^{3}$. This could have influenced the results for radiomic features that are correlated to volume.

Another limitation of current and former studies $[12,13]$ is the phantom itself. The squared shape makes the phantom prone to scatter artefacts around the edges. A new cylindrical version of the CCR phantom is therefore being produced and should be used in future CT-scanner variability studies.

In our study we compared the distribution of radiomic features values compared to two clinical NSCLC patient datasets. One possible explanation for the differences between the two patient datasets is that patient dataset 1 was acquired from multiple hospitals and dataset 2 from a single hospital, which might have resulted in a wider distribution of feature values for dataset 1 . Furthermore, the distribution of feature values shows that not all inserts are very representative for clinical NSCLC datasets. Mackin et al. [13] already showed that the mean and standard deviation in HU of the CCR phantom is different than for patient data. The distribution of HUs in the rubber insert was most comparable to the distribution observed in the NSCLS datasets. However, radiomic features derived from any of the phantom inserts typically had a very limited range when compared to the clinical datasets, as well as a different median value. Inserts with more representative textures are, therefore, warranted for future phantom studies.

In conclusion, this study shows that feature values are influenced by CT-scanner, slice thickness and bin width, whereas the influence of exposure could not be shown. Moreover, the influence of bin width on feature stability was not clear, meaning that we could not indicate an optimal bin width. The test-retest analysis shows that some radiomic features are not robust in a strictly controlled setting: we recommend to exclude those in future radiomic studies. Moreover, we strongly recommend to always perform the pre-processing steps 'resampling' and 'gray-level discretization' for each radiomic study and to clearly report the settings that have been used to improve consistency and reproducibility of radiomic analyses. 


\section{References}

[1] P. Lambin et al. "Radiomics: extracting more information from medical images using advanced feature analysis". In: Eur J Cancer 48.4 (2012). European journal of cancer (Oxford, England : 1990), pp. 441-6. DOI: 10.1016/j.ejca.2011.11.036.

[2] Y. Zhang et al. "Radiomics-based Prognosis Analysis for Non-Small Cell Lung Cancer". In: Sci Rep 7 (2017). Scientific reports, p. 46349. DOI: $10.1038 /$ srep46349.

[3] R. T. Leijenaar et al. "External validation of a prognostic CT-based radiomic signature in oropharyngeal squamous cell carcinoma". In: Acta Oncol 54.9 (2015). Acta oncologica (Stockholm, Sweden), pp. 1423-9. DOI: 10.3109/0284186x.2015.1061214.

[4] R. T. Leijenaar et al. "Stability of FDG-PET Radiomics features: an integrated analysis of test-retest and inter-observer variability". In: Acta Oncol 52.7 (2013). Acta oncologica (Stockholm, Sweden), pp. 1391-7. DOI: 10.3109/0284186x.2013. 812798 .

[5] H. J. Aerts et al. "Decoding tumour phenotype by noninvasive imaging using a quantitative radiomics approach". In: Nat Commun 5 (2014). Nature communications, p. 4006. DOI: 10.1038/ncomms5006.

[6] T. P. Coroller et al. "CT-based radiomic signature predicts distant metastasis in lung adenocarcinoma". In: Radiother Oncol 114.3 (2015). Radiotherapy and oncology : journal of the European Society for Therapeutic Radiology and Oncology, pp. 345-50. DOI: 10.1016/j.radonc.2015.02.015.

[7] M. Scrivener et al. "Radiomics applied to lung cancer: a review". In: Translational Cancer Research 5.4 (2016), pp. 398-409. URL: http://tcr.amegroups.com/article/ view/8536.

[8] R. T. Larue et al. "Quantitative radiomics studies for tissue characterization: a review of technology and methodological procedures". In: Br J Radiol 90.1070 (2017). The British journal of radiology, p. 20160665. DOI: 10.1259/bjr.20160665.

[9] P. Lambin et al. "Radiomics: the bridge between medical imaging and personalized medicine". In: Nat Rev Clin Oncol 14.12 (2017). Nature reviews. Clinical oncology, pp. 749-762. DOI: $10.1038 /$ nrclinonc.2017.141.

[10] Y. Balagurunathan et al. "Test-retest reproducibility analysis of lung CT image features". In: J Digit Imaging 27.6 (2014). Journal of digital imaging, pp. 805-23. DOI: $10.1007 / \mathrm{s} 10278-014-9716-x$.

[11] L. Lu et al. "Assessing Agreement between Radiomic Features Computed for Multiple CT Imaging Settings". In: PLoS One 11.12 (2016). PloS one, e0166550. DOI: 10. 1371/journal. pone.0166550.

[12] M. Shafiq-UI-Hassan et al. "Intrinsic dependencies of CT radiomic features on voxel size and number of gray levels". In: Med Phys 44.3 (2017). Medical physics, pp. 1050-1062. DOI: $10.1002 / \mathrm{mp} .12123$.

[13] D. Mackin et al. "Measuring Computed Tomography Scanner Variability of Radiomics Features". In: Invest Radiol 50.11 (2015). Investigative radiology, pp. 757-65. DOI: 10.1097/rli.0000000000000180. 
[14] J. E. van Timmeren et al. "Survival prediction of non-small cell lung cancer patients using radiomics analyses of cone-beam CT images". In: Radiother Oncol 123.3 (2017). Radiotherapy and oncology : journal of the European Society for Therapeutic Radiology and Oncology, pp. 363-369. DOI: 10.1016/j.radonc.2017.04.016.

[15] L. I. Lin. "A concordance correlation coefficient to evaluate reproducibility". In: Biometrics 45.1 (1989). Biometrics, pp. 255-68.

[16] J. E. van Timmeren et al. "Test-Retest Data for Radiomics Feature Stability Analysis: Generalizable or Study-Specific?" In: Tomography 2.4 (2016). Tomography (Ann Arbor, Mich.), pp. 361-365. DOI: 10.18383/j.tom.2016.00208.

[17] B. Zhao et al. "Reproducibility of radiomics for deciphering tumor phenotype with imaging". In: Sci Rep 6 (2016). Scientific reports, p. 23428. DOI: 10.1038/srep23428.

[18] R. T. Leijenaar et al. "The effect of SUV discretization in quantitative FDG-PET Radiomics: the need for standardized methodology in tumor texture analysis". In: Sci Rep 5 (2015). Scientific reports, p. 11075. DOI: 10.1038/srep11075. 


\section{Supplementary Material}

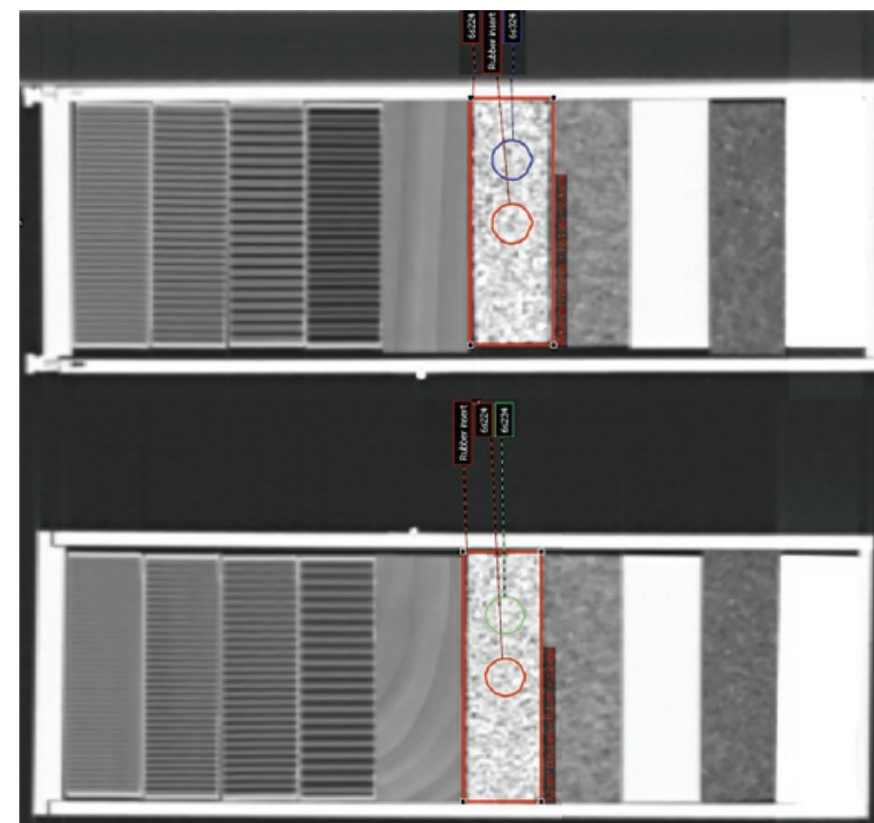

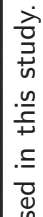
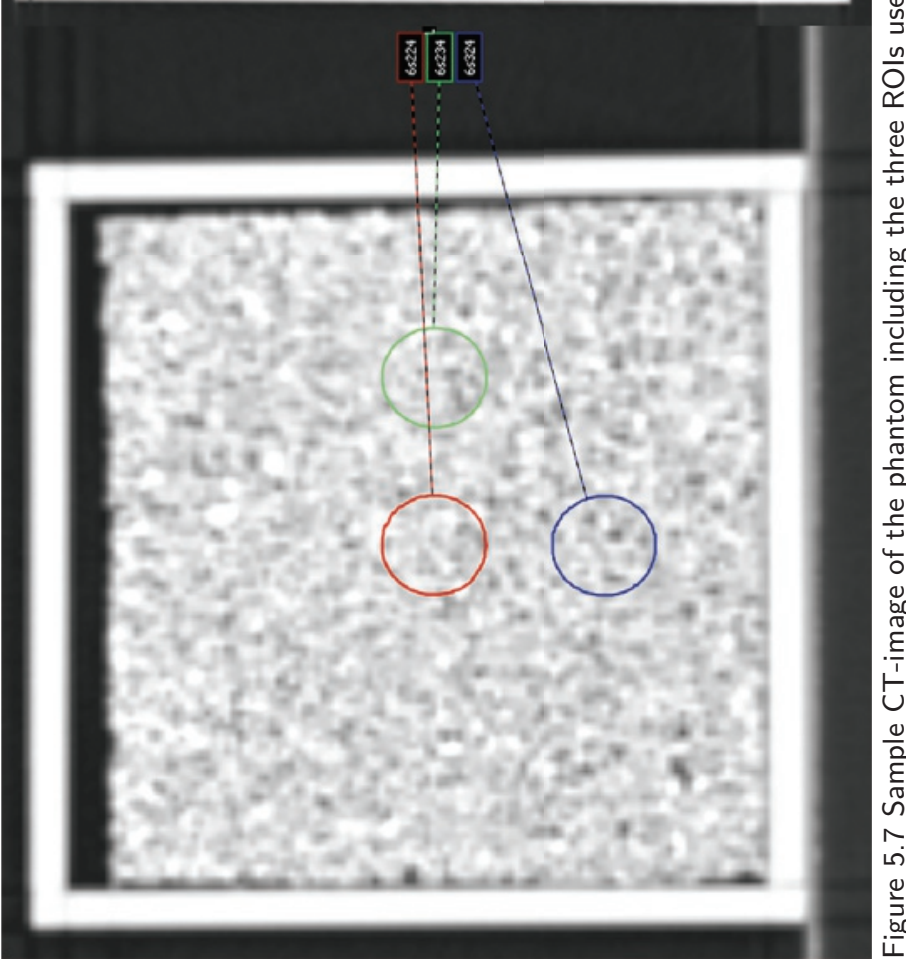
1. Stats_energy

2. GLRLM_RLN

3. GLCM_sumEntro

4. GLRLM_GLN

5. GLCM_sumVar

6. GLCM_clusTend

7. GLCM_entrop2

8. NGTDM_coarseness

9. Stats_entropy

10. Stats_std

11. NGLDM_GLV

12. GLRLM_GLV

13. Stats_md

14. Stats_var

15. NGLDM_GLN

16. GLRLM_GLNN

17. GLRLM RE

18. NGLDM_DN

19. GLCM_energy

20. NGLDM̄_GLNN

21. GLCM_clusProm

22. GLCM_sumSquares

23. Stats_uniformity

24. GLDZM D_DZE

25. GLCM_diffEntro

26. GLCM_diffAvrg

27. Stats_rmd

28. GLCM_dissimilar

29. GLCM_contrast

30. GLDZM

31. GLCM diffVar

32. NGLDM̄_SM

33. Stats $p \overline{90}$

34. GLCM_homogeneity 1

35. GLDZM̄IN

36. GLCM_homogeneity2

37. Stats $\bar{p} 10$

38. GLSZM_IN

39. GLDZM_SDE

40. GLSZM_SZN

41. GLDZM_DZN

42. GLCM_clusShade

43. Stats_iqr

44. GLDZM_DZNN

45. GLDZM-DZV

46. GLCM_inverseVar

47. GLDZM̄IIV

48. GLDZM_INN

49. GLSZM_IV

50. NGTDM_complexity

51. GLDZM HILDE

52. GLSZM_INN

53. Stats_range

54. GLCM_maxProb

55. GLCM_correl1

56. NGLDM̄_DE

57. Stats_max
58. Stats_min
59. GLCM average
60. GLCM_sumAvg
61. GLRLM_SRHGE
62. NGLDM_HGSDE
63. NGLDM LGLDE
64. GLRLM HGRE
65. GLDZM_LISDE
66. NGLDM_HGE
67. Stats_totalenergy
68. Stats rms
69. GLDZM_HIE
70. GLRLM_LRLGE
71. GLSZM_HIE
72. GLCM_autocorr
73. NGLDM̄_LGE
74. GLRLM_LRHGE
75. GLRLM_LGRE
76. GLRLM_SRLGE
77. GLDZM_LIE
78. GLSZM_HISAE
79. GLSZM_LIE
80. GLRLM_SRE
81. GLSZM_LISAE
82. GLRLM_RLNN
83. GLRLM_RP
84. GLSZM-ZE
85. GLDZM_ZP
86. GLRLM_LRE
87. GLSZM_ZP
88. NGLDM_LDE
89. NGLDM_SDE
90. NGLDM_DNN
91. GLSZM_LILAE
92. GLDZM_HISDE
93. NGLDM_LGSDE
94. GLRLM_RLV
95. GLCM_infoCorr2
96. GLCM_infoCorr1
97. NGLDM̄_HGLDE
98. NGTDM contrast
99. GLCM_invDiffmomnor
100. GLCM_invDiffnorm
101. Stats_median
102. GLSZM_LAE
103. GLDZM_LILDE
104. NGLDM_DV
105. GLCM_maxCorr
106. GLSZM_SZV
107. Stats mean
108. NGTDM_busyness
109. NGTDM_strength
110. Stats_skewness
111. Stats_kurtosis
112. GLSZM_HILAE
113. GLSZM_SZ

Figure 5.8 Feature ranking based on the ratio between variations due to slice thickness and due to exposure. This ranking is the sum ranking over all scanners. 

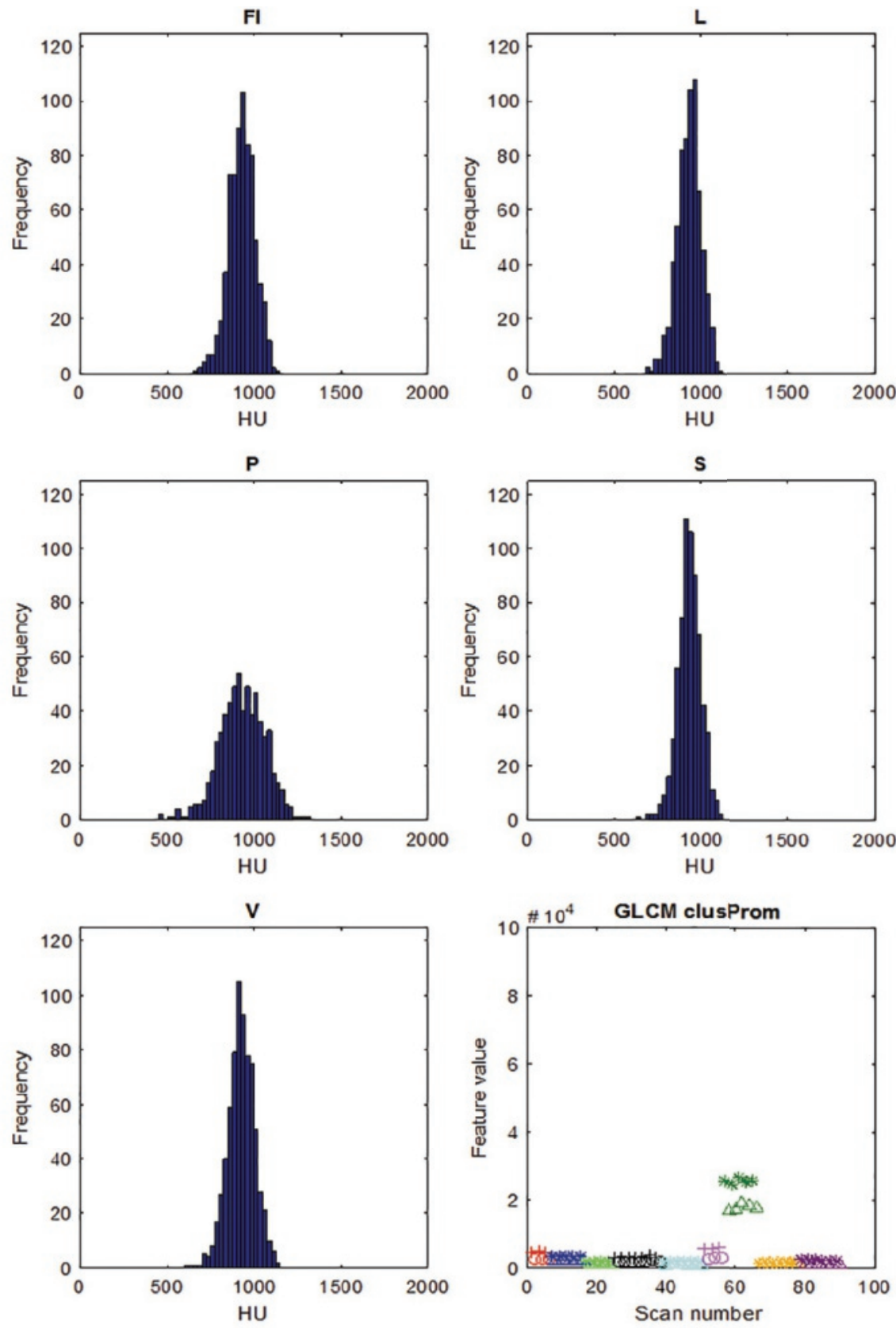

Figure $5.9 \mathrm{HU}$ distributions of the rubber insert scanned with a fixed CTDI of $5.43 \mathrm{mGy}$ on five different scanners, after resampling to voxels to $1 \times 1 \times 3 \mathrm{~mm} 3$ using linear interpolation. From left to right and top to bottom [scanner - tube current]: 'Fl-160 mA', 'L-161 mA', 'P-148 mA', 'S-143 mA', and ' $\mathrm{V}-162 \mathrm{~mA}$ '. The scatterplot in the bottom right displays the observed values of the feature 'GLCM cluster prominence' over all scanners. The outlier in dark-green is scanner ' $\mathrm{P}$ '. 


\begin{tabular}{|c|c|c|c|c|c|c|c|c|c|c|}
\hline \multirow{2}{*}{ Radiomic feature } & \multicolumn{10}{|c|}{ CCC $1.5 \mathrm{~mm}$ vs. $3.0 \mathrm{~mm}$} \\
\hline & BW 5 & BW 10 & BW 15 & BW 20 & BW 25 & BW 30 & BW 35 & BW 40 & BW 45 & BW 50 \\
\hline GLCM_autocorr & 0,66 & 0,65 & 0,65 & 0,63 & 0,68 & 0,65 & 0,67 & 0,69 & 0,63 & 0,70 \\
\hline GLCM_average & 0,83 & 0,83 & 0,82 & 0,81 & 0,83 & 0,81 & 0,83 & 0,85 & 0,81 & 0,81 \\
\hline GLCM_clusProm & 0,66 & 0,65 & 0,65 & 0,64 & 0,64 & 0,64 & 0,62 & 0,62 & 0,63 & 0,62 \\
\hline GLCM_clusshade & 0,69 & 0,69 & 0,70 & 0,69 & 0,68 & 0,71 & 0,67 & 0,70 & 0,74 & 0,70 \\
\hline GLCM_clusTend & 0,90 & 0,90 & 0,90 & 0,90 & 0,90 & 0,90 & 0,89 & 0,89 & 0,89 & 0,90 \\
\hline GLCM_contrast & 0,93 & 0,93 & 0,93 & 0,93 & 0,93 & 0,93 & 0,93 & 0,92 & 0,93 & 0,93 \\
\hline GLCM_correl1 & 0,91 & 0,90 & 0,90 & 0,90 & 0,89 & 0,91 & 0,89 & 0,89 & 0,90 & 0,90 \\
\hline GLCM_diffAvrg & 0,96 & 0,96 & 0,96 & 0,96 & 0,96 & 0,96 & 0,96 & 0,96 & 0,96 & 0,97 \\
\hline GLCM_diffEntro & 0,96 & 0,96 & 0,96 & 0,97 & 0,97 & 0,97 & 0,97 & 0,97 & 0,97 & 0,96 \\
\hline GLCM_diffVar & 0,92 & 0,92 & 0,92 & 0,92 & 0,92 & 0,92 & 0,92 & 0,92 & 0,92 & 0,93 \\
\hline GLCM_dissimilar & 0,96 & 0,96 & 0,96 & 0,96 & 0,96 & 0,96 & 0,96 & 0,96 & 0,96 & 0,97 \\
\hline GLCM_energy & 0,74 & 0,79 & 0,81 & 0,85 & 0,88 & 0,83 & 0,95 & 0,96 & 0,93 & 0,91 \\
\hline GLCM_entrop2 & 0,75 & 0,89 & 0,92 & 0,93 & 0,94 & 0,94 & 0,95 & 0,95 & 0,95 & 0,95 \\
\hline GLCM_homogeneity1 & 0,95 & 0,96 & 0,96 & 0,96 & 0,97 & 0,97 & 0,97 & 0,97 & 0,97 & 0,97 \\
\hline GLCM_homogeneity2 & 0,93 & 0,95 & 0,96 & 0,96 & 0,97 & 0,97 & 0,97 & 0,97 & 0,97 & 0,97 \\
\hline GLCM_infoCorr1 & 0,83 & 0,85 & 0,92 & 0,96 & 0,97 & 0,99 & 0,98 & 0,97 & 0,99 & 0,99 \\
\hline GLCM_infoCorr2 & 0,99 & 0,98 & 0,98 & 0,98 & 0,98 & 0,98 & 0,98 & 0,96 & 0,97 & 0,96 \\
\hline GLCM_invDiffmomnor & 0,96 & 0,96 & 0,95 & 0,95 & 0,96 & 0,96 & 0,93 & 0,78 & 0,81 & 0,96 \\
\hline GLCM_invDiffnorm & 0,94 & 0,94 & 0,94 & 0,95 & 0,95 & 0,97 & 0,94 & 0,88 & 0,91 & 0,96 \\
\hline GLCM_inverseVar & 0,93 & 0,95 & 0,95 & 0,95 & 0,95 & 0,93 & 0,98 & 0,98 & 0,97 & 0,88 \\
\hline GLCM_maxCorr & 0,88 & 0,95 & 0,95 & 0,95 & 0,92 & 0,95 & 0,92 & 0,89 & 0,90 & 0,92 \\
\hline GLCM_! & 0,91 & 0,83 & 0,82 & 0,90 & 0,91 & 0,90 & 0,93 & 0,96 & 0,94 & 0,95 \\
\hline GLCM_sumAvg & 0,83 & 0,83 & 0,82 & 0,81 & 0,83 & 0,81 & 0,83 & 0,85 & 0,81 & 0,81 \\
\hline GLCM_sumEntro & 0,92 & 0,93 & 0,94 & 0,94 & 0,94 & 0,94 & 0,94 & 0,94 & 0,94 & 0,93 \\
\hline GLCM_sumSquares & 0,91 & 91 & 0,91 & 0,91 & 0,91 & 0,91 & 0,91 & 0,90 & 0,91 & 0,91 \\
\hline GLCM_sumVar & 0,90 & 0,90 & 0,90 & 0,90 & 0,90 & 0,90 & 0,89 & 0,89 & 0,89 & 0,90 \\
\hline GLDZN & 0,63 & 0 , & 0,68 & 0,67 & 0,69 & 0,73 & 0,69 & 0,60 & 0,70 & 0,64 \\
\hline GLDZM_DZN & 0,64 & 0,86 & 0,93 & 0,94 & 0,95 & 0,93 & 0,94 & 0,93 & 0,95 & 0,95 \\
\hline GLDZM_DZNN & 0,07 & 0,09 & $\underline{0,06}$ & $\underline{0.04}$ & $\underline{0,10}$ & $\underline{0,19}$ & $\underline{0.04}$ & $\underline{0,10}$ & 0,14 & $\underline{0,39}$ \\
\hline GLDZN & $\underline{0.02}$ & $\underline{0.02}$ & 0.00 & $\underline{0.02}$ & $\underline{0.02}$ & $\underline{0.04}$ & 0.04 & $\underline{0.06}$ & $\underline{0.05}$ & $\underline{0.14}$ \\
\hline GLDZM_HIE & 0,66 & 0,66 & 0,66 & 0,64 & 0,68 & 0,65 & 0,67 & 0,68 & 0,60 & 0,68 \\
\hline GLDZM_HILDE & 0,35 & 0,33 & 0,35 & 0,33 & 0,36 & 0,34 & 0,36 & 0,38 & 0,32 & 0,38 \\
\hline GLDZM_HISDE & 0,84 & 0 , & 0,82 & 0,81 & 0,84 & 0,80 & 0,81 & 0,82 & 0,75 & 0,81 \\
\hline GLDZM_IN & 0,32 & 0,22 & 0,27 & 0,45 & 0,46 & 0,45 & 0,31 & 0,56 & 0,57 & $\underline{0.53}$ \\
\hline GLDZN & 0,88 & & 0,89 & 0,90 & 0,89 & 0,97 & 0,90 & 0,89 & 0,98 & 0,93 \\
\hline GLDZM_IV & 0,90 & 0,90 & 0,89 & 0,87 & 0,86 & 0,87 & 0,88 & 0,84 & 0,82 & 0,83 \\
\hline GLDZM_LDE & 0,04 & 0,04 & $\underline{0.03}$ & $\underline{0.03}$ & $\underline{0.05}$ & $\underline{0.07}$ & $\underline{0.00}$ & 0,03 & $\underline{0.06}$ & $\underline{0,17}$ \\
\hline GLDZM_LIE & 0,64 & 0,30 & 0.16 & 0,19 & 0.14 & 0.44 & 0.47 & 0,88 & 0,80 & 0.06 \\
\hline GLDZM_LILDE & $\underline{0.69}$ & $\underline{0.46}$ & 0.23 & 0.43 & 0,27 & $\underline{0,85}$ & $\underline{0.73}$ & $\underline{0.45}$ & $\underline{0.17}$ & $\underline{0,34}$ \\
\hline GLDZM_LISDE & 0,62 & 0,27 & 0,14 & 0,13 & $\underline{0,11}$ & $\underline{0,34}$ & $\underline{0.28}$ & 0,49 & 0,83 & $\underline{0.02}$ \\
\hline GLDZM_SDE & 0,10 & 0,10 & $\underline{0.08}$ & $\underline{0.06}$ & $\underline{0.11}$ & $\underline{0.17}$ & $\underline{-0.01}$ & 0,04 & 0,08 & $\underline{0.31}$ \\
\hline GLDZM_ZP & 0,97 & 0,96 & 0,93 & 0,92 & 0,89 & 0,87 & 0,85 & 0,86 & 0,81 & 0,81 \\
\hline GLRLM_GLN & 0,72 & 0,69 & 0,65 & 0,60 & 0,55 & 0,47 & 0,48 & 0,44 & 0,39 & 0,28 \\
\hline
\end{tabular}




\begin{tabular}{|c|c|c|c|c|c|c|c|c|c|c|}
\hline \multirow{2}{*}{ Radiomic feature } & \multicolumn{10}{|c|}{ CCC $1.5 \mathrm{~mm}$ vs. $3.0 \mathrm{~mm}$} \\
\hline & BW 5 & BW 10 & BW 15 & BW 20 & BW 25 & BW 30 & BW 35 & BW 40 & BW 45 & BW 50 \\
\hline GLRLM_GLNN & 0,89 & 0,90 & 0,91 & 0,92 & 0,93 & 0,93 & 0,95 & 0,96 & 0,95 & 0,85 \\
\hline GLRLM_GLV & 0,91 & 0,91 & 0,91 & 0,91 & 0,91 & 0,90 & 0,90 & 0,90 & 0,90 & 0,90 \\
\hline GLRLM_HGRE & 0,66 & 0,66 & 0,66 & 0,64 & 0,69 & 0,66 & 0,67 & 0,69 & 0,63 & 0,70 \\
\hline GLRLM_LGRE & 0,99 & 0,92 & 0,75 & 0,61 & 0,56 & $\underline{0,30}$ & $\underline{0,86}$ & 0,47 & 0,43 & $\underline{0,57}$ \\
\hline GLRLM_LRE & 0,91 & 0,91 & 0,91 & 0,91 & 0,95 & 0,86 & 0,95 & 0,95 & 0,93 & 0,92 \\
\hline GLRLM_LRHGE & 0,66 & 0,66 & 0,65 & 0,62 & 0,67 & 0,62 & 0,64 & 0,66 & 0,57 & 0,59 \\
\hline GLRLM_LRLGE & 0,98 & 0,93 & 0,83 & 0,69 & 0,59 & 0,36 & $\underline{0.85}$ & 0,55 & 0,51 & 0.55 \\
\hline GLRLM_RE & 0,93 & 0,93 & 0,92 & 0,91 & 0,89 & 0,88 & 0,85 & 0,81 & 0,78 & 0,53 \\
\hline GLRLM_RLN & 0,02 & 0,07 & 0,12 & 0,18 & 0,22 & 0,28 & 0,28 & 0,31 & 0,35 & 0,43 \\
\hline GLRLM_RLNN & 0,96 & 0,96 & 0,97 & 0,97 & 0,97 & 0,97 & 0,98 & 0,98 & 0,98 & 0,98 \\
\hline GLRLM_RLV & 0,83 & 0,87 & 0,90 & 0,90 & 0,95 & 0,86 & 0,94 & 0,95 & 0,93 & 0,98 \\
\hline GLRLM_RP & 0,95 & 0,96 & 0,97 & 0,97 & 0,98 & 0,97 & 0,98 & 0,97 & 0,98 & 0,98 \\
\hline GLRLM_SRE & 0,95 & 0,95 & 0,96 & 0,96 & 0,97 & 0,96 & 0,98 & 0,98 & 0,98 & 0,93 \\
\hline GLRLM_SRHGE & 0,66 & 0,65 & 0,66 & 0,64 & 0,69 & 0,66 & 0,67 & 0,69 & 0,63 & 0,71 \\
\hline GLRLM_SRLGE & 0,99 & 0,89 & 0,69 & 0,55 & 0,51 & $\underline{0.25}$ & $\underline{0,85}$ & 0,44 & 0,44 & $\underline{0,54}$ \\
\hline GLSZM_HIE & 0,66 & 0,66 & 0,66 & 0,64 & 0,68 & 0,65 & 0,67 & 0,68 & 0,60 & 0,68 \\
\hline GLSZM_HILAE & 0,84 & 0,67 & 0,66 & 0,68 & 0,40 & 0,52 & 0,49 & 0,89 & 0,51 & 0,51 \\
\hline GLSZM_HISAE & 0,66 & 0,64 & 0,66 & 0,62 & 0,66 & 0,66 & 0,71 & 0,67 & 0,57 & 0,72 \\
\hline GLSZM_IN & 0,32 & 0,22 & 0,27 & 0,45 & 0,46 & 0,45 & 0,31 & 0,56 & 0,57 & 0,53 \\
\hline GLSZM_INN & 0,88 & 0,88 & 0,89 & 0,90 & 0,89 & 0,97 & 0,90 & 0,89 & 0,98 & 0,93 \\
\hline GLSZM_IV & 0,90 & 0,90 & 0,89 & 0,87 & 0,86 & 0,87 & 0,88 & 0,84 & 0,82 & 0,83 \\
\hline GLSZM_LAE & 0,95 & 0,96 & 0,90 & 0,88 & 0,77 & 0,82 & 0,32 & 0,58 & 0,96 & 0,80 \\
\hline GLSZM_LIE & 0,64 & 0,30 & $\underline{0,16}$ & 0,19 & 0,14 & 0,44 & 0,47 & 0,88 & 0,80 & 0,06 \\
\hline GLSZM_LILAE & 0,73 & 0,46 & $\underline{0.37}$ & 0,66 & 0.50 & 0.51 & 0.15 & 0,18 & 0,41 & 0.78 \\
\hline GLSZM_LISAE & 0,32 & 0,21 & 0,11 & $\underline{0.18}$ & 0,17 & 0,28 & 0.65 & $-0,22$ & $\underline{0.05}$ & 0.10 \\
\hline GLSZM_SAE & 0,93 & 0,85 & 0,30 & 0,66 & 0,67 & 0,81 & 0,13 & $-0,18$ & 0,36 & 0,95 \\
\hline GLSZM_SZN & 0,42 & 0,52 & 0,61 & 0,58 & 0,56 & 0,61 & 0,65 & 0,55 & 0,53 & 0,67 \\
\hline GLSZM_SZNN & 0,94 & 0,86 & $\underline{0.48}$ & 0,73 & 0.65 & 0,83 & 0,45 & 0,89 & 0,66 & 0,93 \\
\hline GLSZM_SZV & 0,95 & 0,96 & 0,89 & 0,86 & 0,76 & 0,81 & 0,66 & 0,20 & 0,74 & 0,77 \\
\hline GLSZM_ZE & 0,77 & 0,84 & 0,85 & 0,86 & 0,93 & 0,90 & 0,96 & 0,92 & 0,95 & 0,93 \\
\hline GLSZM_ZP & 0,97 & 0,96 & 0,93 & 0,92 & 0,89 & 0,87 & 0,85 & 0,86 & 0,81 & 0,81 \\
\hline NGLDM_DE & 0,73 & 0,79 & 0,78 & 0,79 & 0,79 & 0,83 & 0,86 & 0,82 & 0,75 & 0,83 \\
\hline NGLDM_DN & 0,32 & 0,34 & 0,36 & 0,37 & 0,37 & 0,36 & 0,30 & 0,31 & 0,34 & 0,32 \\
\hline NGLDM_DNN & 0,94 & 0,93 & 0,95 & 0,95 & 0,95 & 0,94 & 0,95 & 0,94 & 0,96 & 0,96 \\
\hline NGLDM_DV & 0,96 & 0,94 & 0,91 & 0,95 & 0,96 & 0,95 & 0,95 & 0,96 & 0,96 & 0,95 \\
\hline NGLDM_GLN & 0,73 & 0,74 & 0,74 & 0,71 & 0,68 & 0,72 & 0,57 & 0,53 & 0,57 & 0,66 \\
\hline NGLDM_GLNN & 0,89 & 0,90 & 0,91 & 0,93 & 0,94 & 0,92 & 0,96 & 0,96 & 0,95 & 0,95 \\
\hline NGLDM_GLV & 0,91 & 0,91 & 0,91 & 0,91 & 0,91 & 0,91 & 0,91 & 0,91 & 0,91 & 0,91 \\
\hline NGLDM_HGE & 0,66 & 0,66 & 0,65 & 0,64 & 0,69 & 0,66 & 0,67 & 0,69 & 0,63 & 0,70 \\
\hline NGLDM_HGLDE & 0,66 & 0,66 & 0,59 & 0,56 & 0,57 & $\underline{0,55}$ & $\underline{0,55}$ & 0,76 & 0,62 & $\underline{0,53}$ \\
\hline NGLDM_HGSDE & 0,66 & 0,62 & 0,64 & 0,59 & 0,62 & 0,59 & 0,63 & 0,60 & 0,53 & 0,69 \\
\hline NGLDM_LDE & 0,94 & 0,94 & 0,95 & 0,97 & 0,98 & 0,95 & 0,97 & 0,95 & 0,97 & 0,97 \\
\hline NGLDM_LGE & 0,99 & 0,95 & 0,85 & 0,74 & 0,69 & 0,46 & 0,90 & 0,51 & 0,43 & 0.73 \\
\hline
\end{tabular}




\begin{tabular}{|c|c|c|c|c|c|c|c|c|c|c|}
\hline \multirow{2}{*}{ Radiomic feature } & \multicolumn{10}{|c|}{ CCC $1.5 \mathrm{~mm}$ vs. $3.0 \mathrm{~mm}$} \\
\hline & BW 5 & BW 10 & BW 15 & BW 20 & BW 25 & BW 30 & BW 35 & BW 40 & BW 45 & BW 50 \\
\hline NGLDM_LGLDE & 0,99 & 0,97 & 0,92 & 0,77 & 0,63 & 0,40 & 0,88 & 0,56 & 0,46 & $\underline{0,66}$ \\
\hline NGLDM_LGSDE & 0,73 & $\underline{0,43}$ & 0.23 & 0,21 & 0,34 & 0,49 & 0.69 & 0,76 & 0,74 & 0,26 \\
\hline NGLDM_SDE & 0,96 & 0,94 & 0,92 & 0,93 & 0,91 & 0,91 & 0,92 & 0,93 & 0,91 & 0,94 \\
\hline NGLDM_SM & 0,44 & 0,40 & 0,41 & 0,34 & 0,35 & 0,31 & 0,26 & 0,23 & 0,27 & 0,45 \\
\hline NGTDM_busyness & 0,77 & 0,75 & 0,51 & 0,63 & $\underline{0,72}$ & $\underline{0.45}$ & $\underline{0.73}$ & 0,27 & $\underline{0.00}$ & $\underline{0.44}$ \\
\hline NGTDM_coarseness & 0,35 & 0,30 & 0,28 & 0,28 & 0,23 & 0,25 & 0,23 & 0,23 & 0,25 & 0,30 \\
\hline NGTDM_complexity & 0,69 & 0,63 & 0,63 & 0,60 & 0,61 & 0,58 & 0,56 & 0,56 & 0,49 & 0,59 \\
\hline NGTDM_contrast & 1,00 & 1,00 & 1,00 & 0,99 & 1,00 & 1,00 & 0,99 & 0,96 & 0,98 & 0,99 \\
\hline NGTDM_strength & 0,90 & 0,93 & 0,94 & 0,95 & 0,96 & 0,94 & 0,95 & 0,95 & 0,90 & 0,95 \\
\hline Stats_energy & 0,70 & 0,70 & 0,70 & 0,70 & 0,70 & 0,70 & 0,70 & 0,70 & 0,70 & 0,70 \\
\hline Stats_entropy & 0,94 & 0,95 & 0,95 & 0,95 & 0,95 & 0,95 & 0,96 & 0,96 & 0,96 & 0,95 \\
\hline Stats_iqr & 0,98 & 0,98 & 0,98 & 0,98 & 0,98 & 0,98 & 0,98 & 0,98 & 0,98 & 0,98 \\
\hline Stats_kurtosis & 0,95 & 0,95 & 0,95 & 0,95 & 0,95 & 0,95 & 0,95 & 0,95 & 0,95 & 0,95 \\
\hline Stats_max & 0,99 & 0,99 & 0,99 & 0,99 & 0,99 & 0,99 & 0,99 & 0,99 & 0,99 & 0,99 \\
\hline Stats_md & 0,96 & 0,96 & 0,96 & 0,96 & 0,96 & 0,96 & 0,96 & 0,96 & 0,96 & 0,96 \\
\hline Stats_mean & 1,00 & 1,00 & 1,00 & 1,00 & 1,00 & 1,00 & 1,00 & 1,00 & 1,00 & 1,00 \\
\hline Stats_median & 1,00 & 1,00 & 1,00 & 1,00 & 1,00 & 1,00 & 1,00 & 1,00 & 1,00 & 1,00 \\
\hline Stats_min & 0,99 & 0,99 & 0,99 & 0,99 & 0,99 & 0,99 & 0,99 & 0,99 & 0,99 & 0,99 \\
\hline Stats_p10 & 1,00 & 1,00 & 1,00 & 1,00 & 1,00 & 1,00 & 1,00 & 1,00 & 1,00 & 1,00 \\
\hline Stats_p90 & 1,00 & 1,00 & 1,00 & 1,00 & 1,00 & 1,00 & 1,00 & 1,00 & 1,00 & 1,00 \\
\hline Stats_range & 0,82 & 0,82 & 0,82 & 0,82 & 0,82 & 0,82 & 0,82 & 0,82 & 0,82 & 0,82 \\
\hline Stats_rmd & 0,98 & 0,98 & 0,98 & 0,98 & 0,98 & 0,98 & 0,98 & 0,98 & 0,98 & 0,98 \\
\hline Stats_rms & 1,00 & 1,00 & 1,00 & 1,00 & 1,00 & 1,00 & 1,00 & 1,00 & 1,00 & 1,00 \\
\hline Stats_skewness & 0,98 & 0,98 & 0,98 & 0,98 & 0,98 & 0,98 & 0,98 & 0,98 & 0,98 & 0,98 \\
\hline Stats_std & 0,95 & 0,95 & 0,95 & 0,95 & 0,95 & 0,95 & 0,95 & 0,95 & 0,95 & 0,95 \\
\hline Stats_totalenergy & 1,00 & 1,00 & 1,00 & 1,00 & 1,00 & 1,00 & 1,00 & 1,00 & 1,00 & 1,00 \\
\hline Stats_uniformity & 0,89 & 0,90 & 0,91 & 0,93 & 0,94 & 0,92 & 0,96 & 0,96 & 0,95 & 0,95 \\
\hline Stats_var & 0,92 & 0,92 & 0,92 & 0,92 & 0,92 & 0,92 & 0,92 & 0,92 & 0,92 & 0,92 \\
\hline
\end{tabular}

Figure 5.10 Feature stability comparing a slice thickness of $1.5 \mathrm{~mm}$ with $3 \mathrm{~mm}$ (scanner 'B', exposure $60 \mathrm{~mA}$ ). CCC-values $<0.85$ are indicated in red, and values $\geq 0.85$ in green. The cells marked in bold indicate features with a CCC $<0.85$ in at least one of the test-retest scans [scanner - tube current - slice thickness]: 'B-60mA-3mm' or 'B-60mA-1.5mm'. 

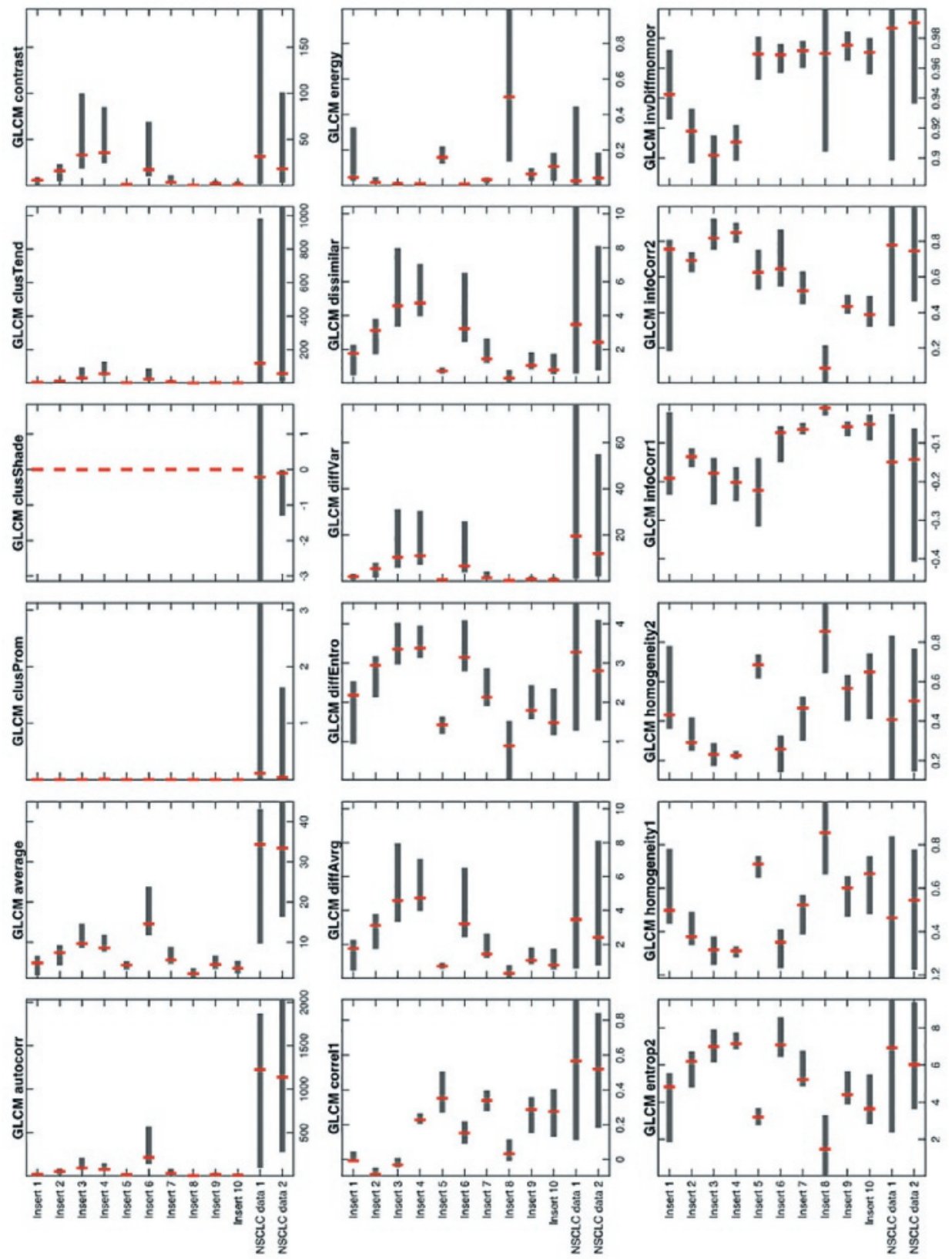

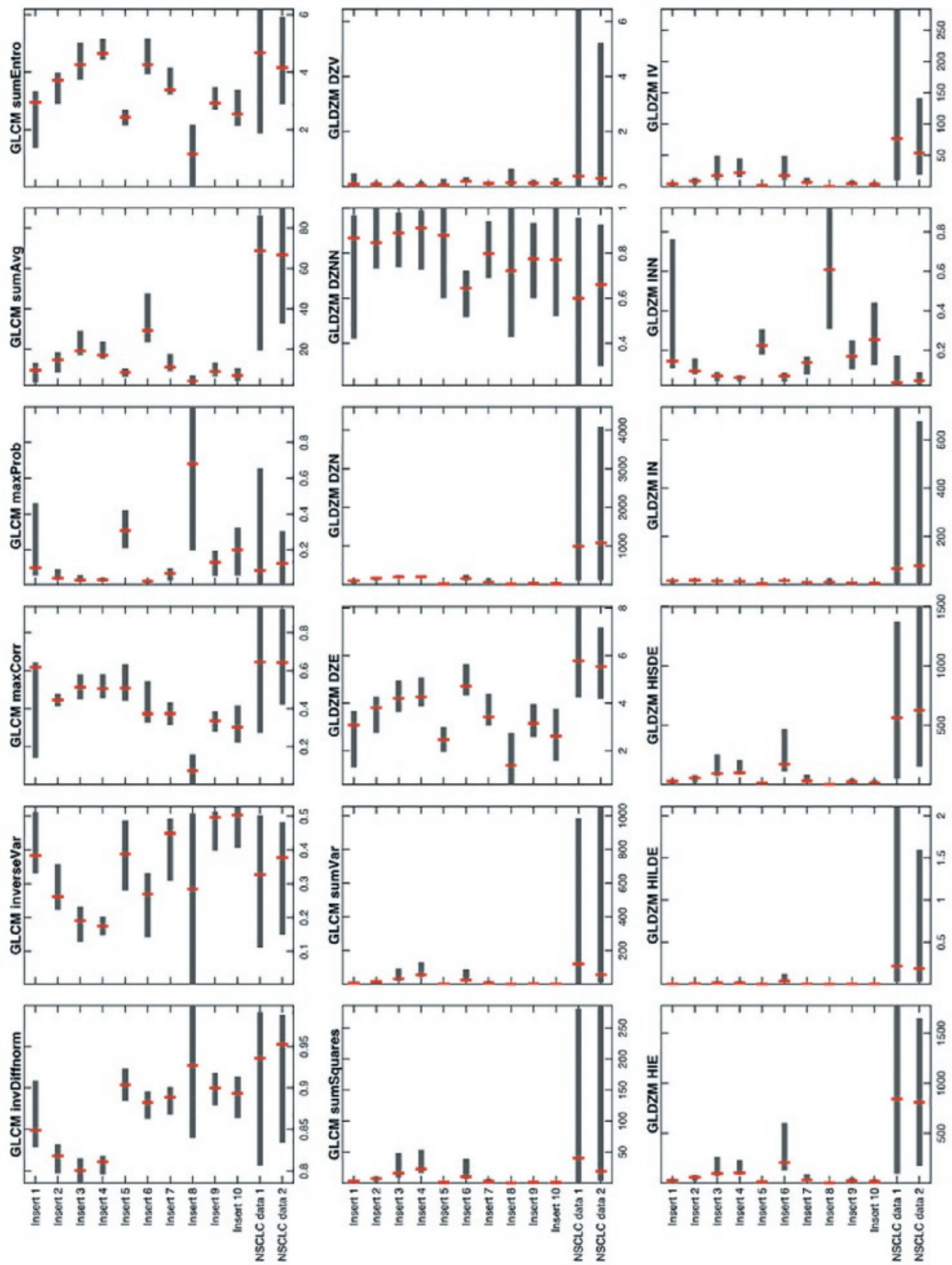

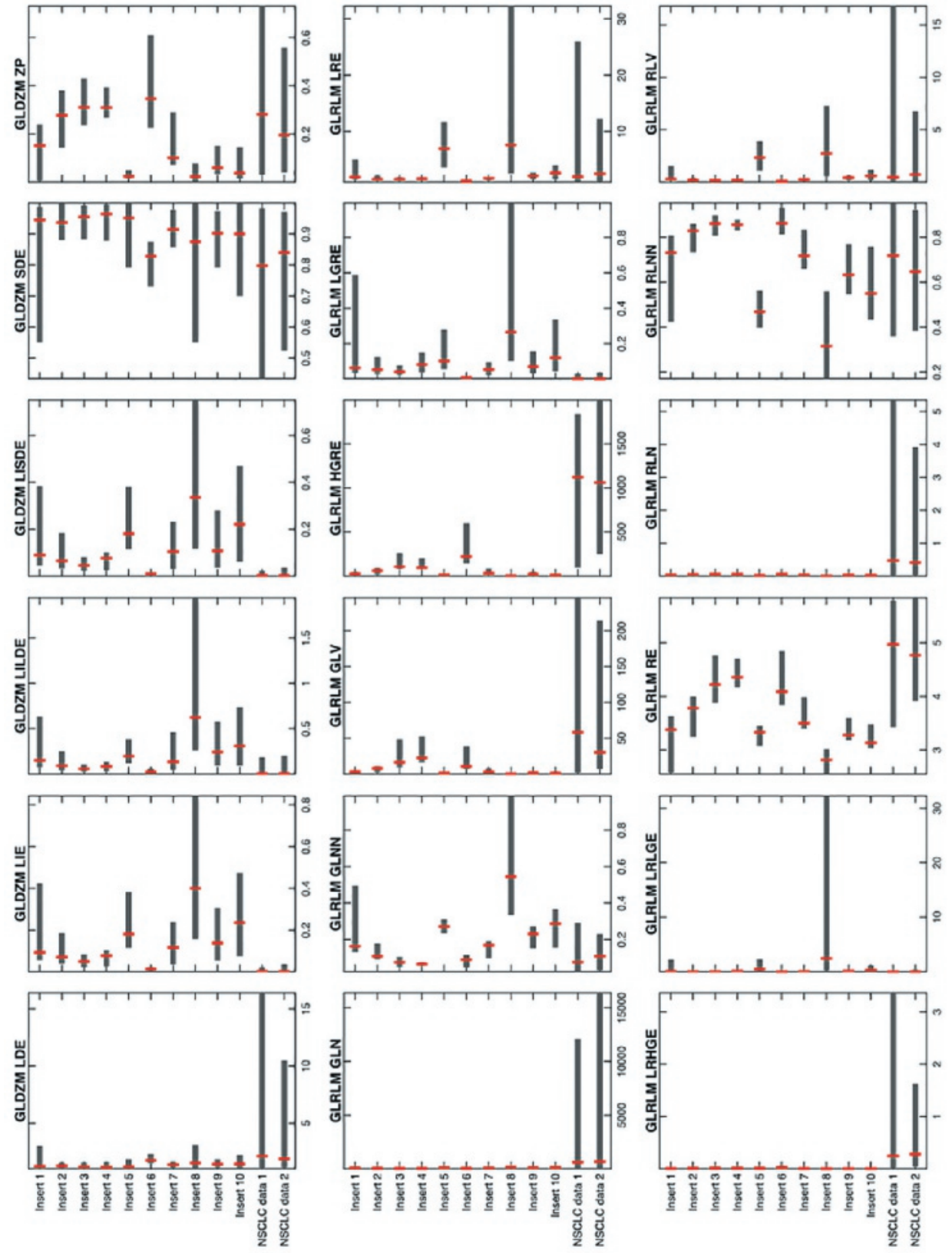

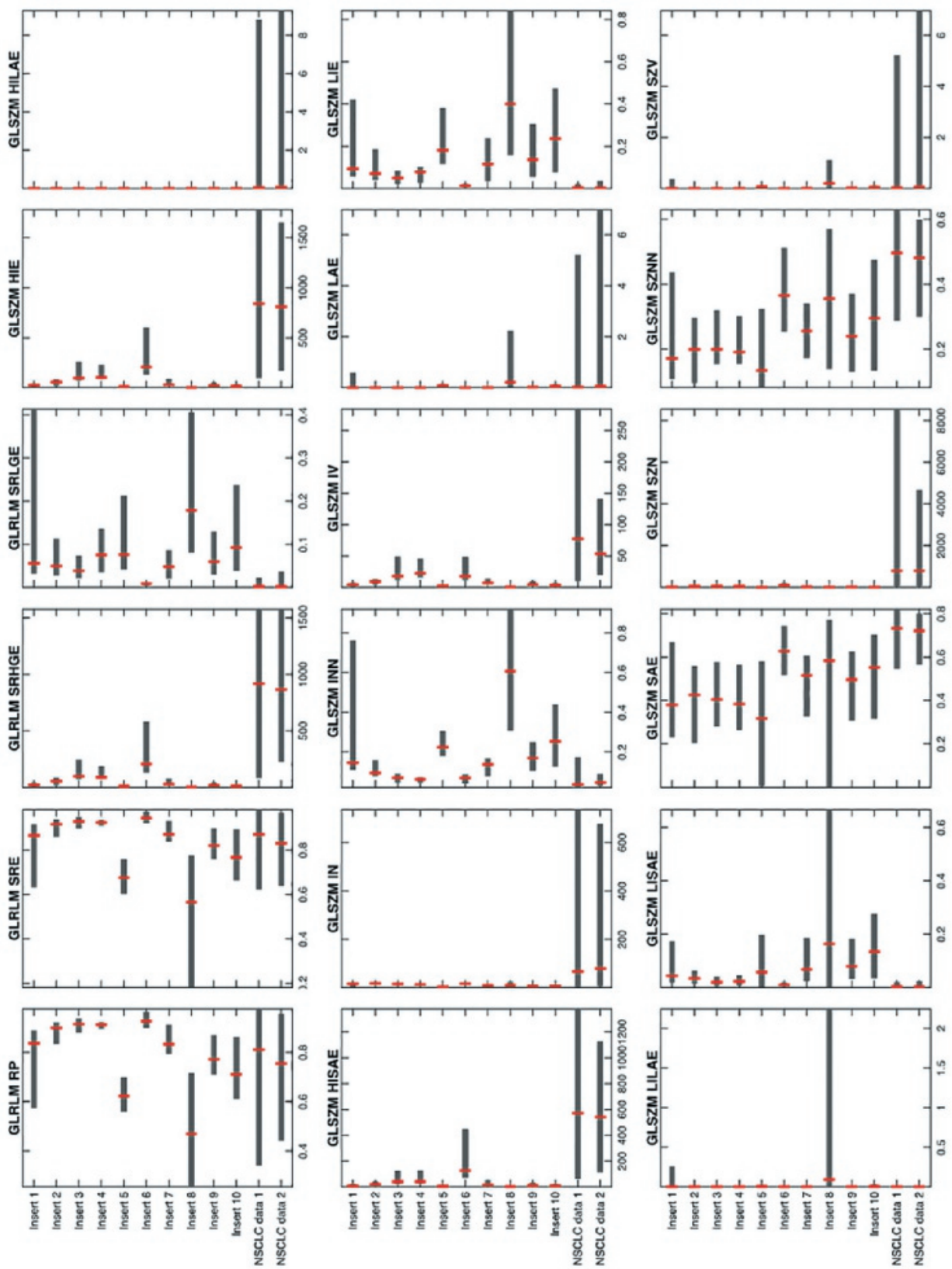

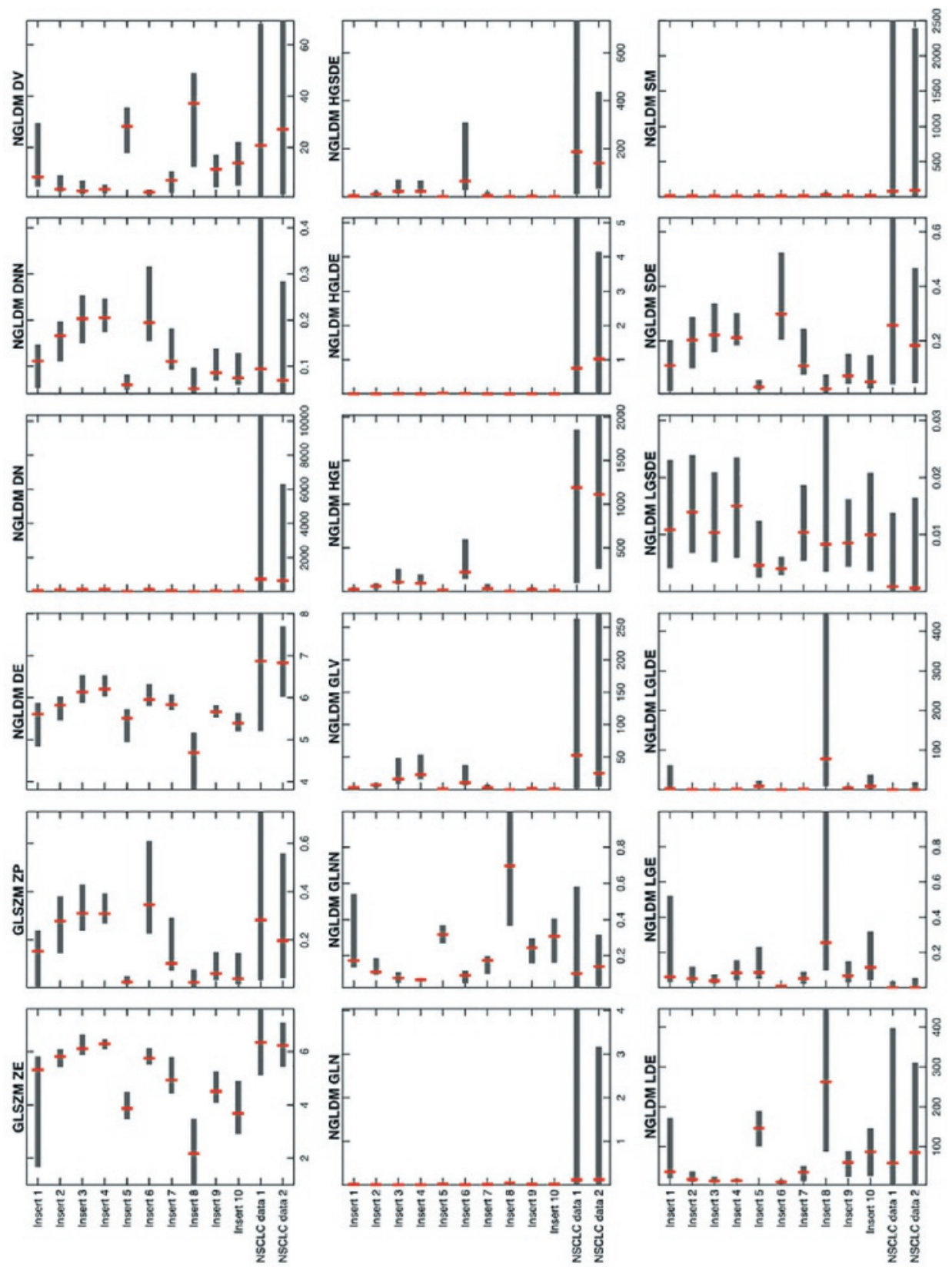

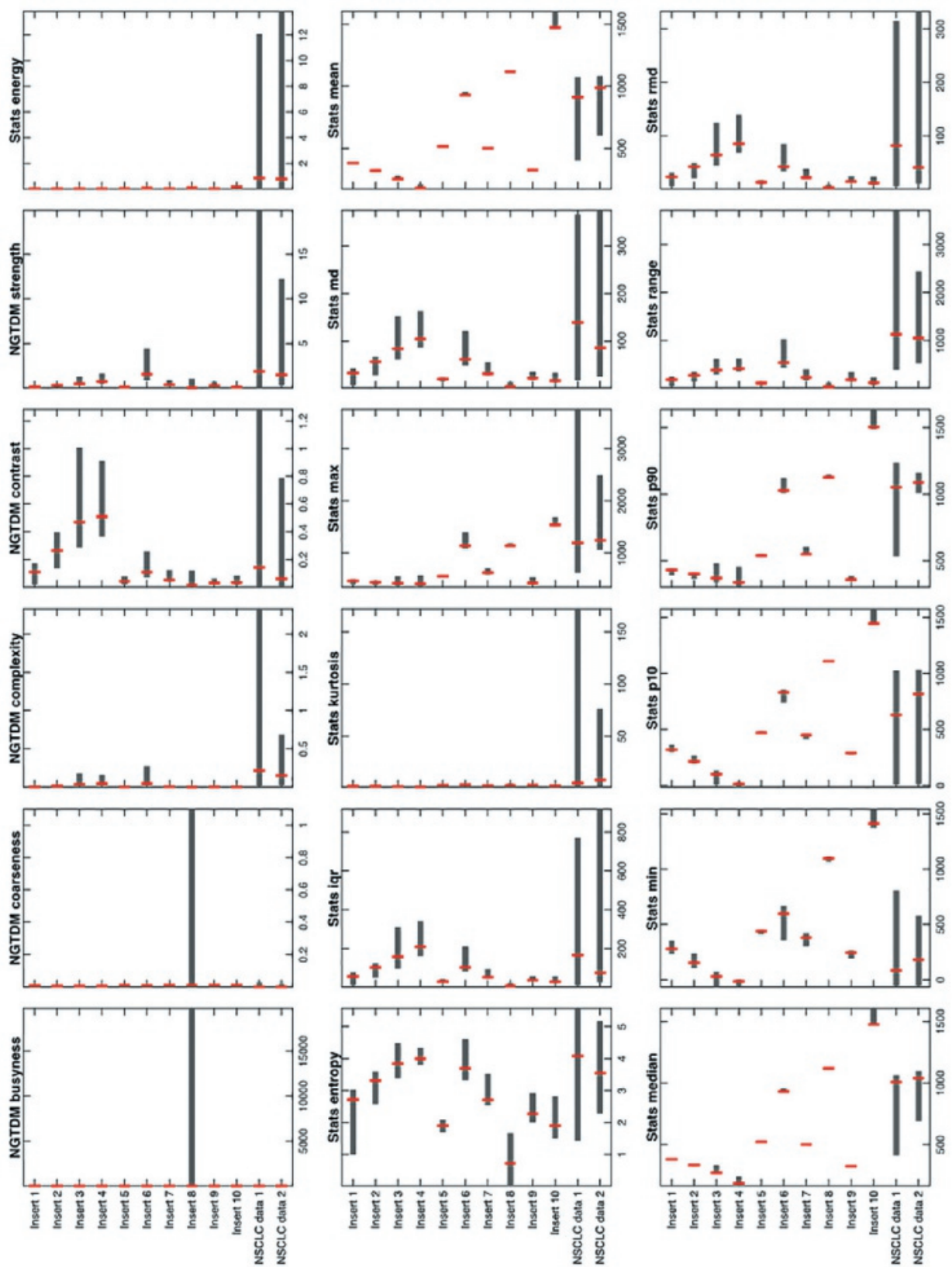


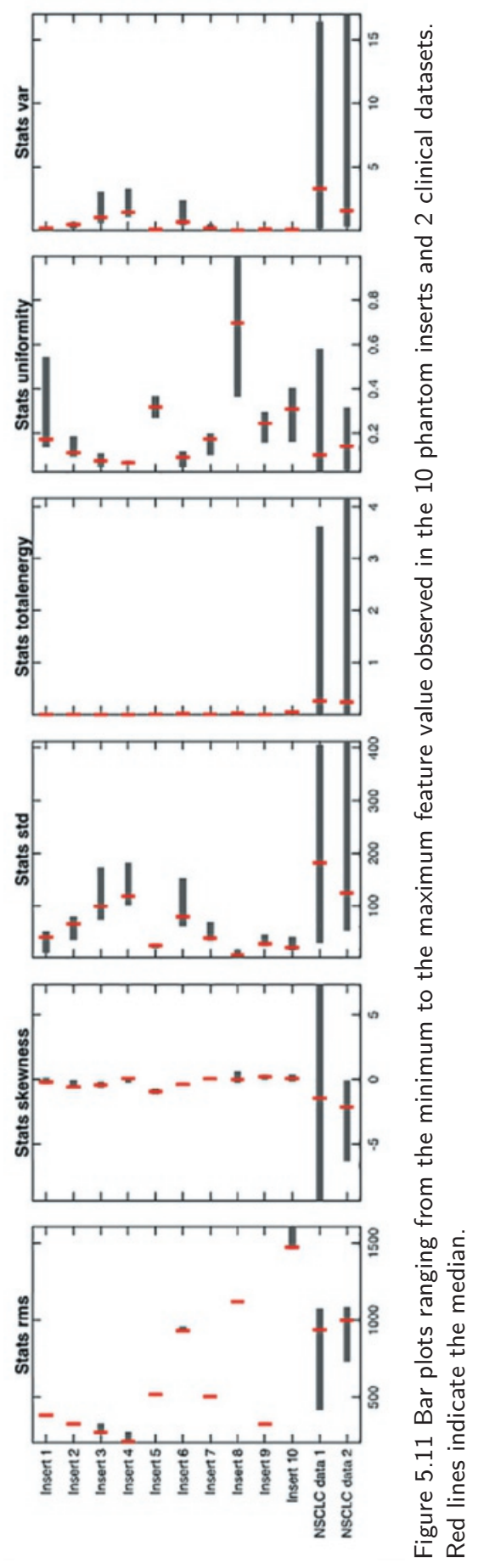



Image features for prognosis and cachexia 



\section{Chapter 6}

${ }^{18}$ F-FDG PET/CT-based response assessment of stage IV non-small cell lung cancer treated with paclitaxel-carboplatin-bevacizumab with or without nitroglycerin patches

Authors

Evelyn E.C. de Jong, Wouter van Elmpt, Ralph T.H. Leijenaar, Otto S. Hoekstra, Harry J.M. Groen, Ebgert F. Smit, Ronald Boellaard, Vincent van der Noort,

Esther G.C. Troost, Philippe Lambin, Anne-Marie C. Dingemans

European Journal of Nuclear Medicine and Molecular Imaging, 2017 January; 44(1): 8-16 DOI: $10.1007 /$ s00259-016-3498-y 


\section{Abstract}

\section{Purpose}

Nitroglycerin (NTG) is a vasodilating drug, which increases tumor blood flow and consequently decreases hypoxia. Therefore, changes in ${ }^{18} \mathrm{~F}$-fluorodeoxyglucose positron emission tomography $\left({ }^{18} \mathrm{~F}-\mathrm{FDG} \mathrm{PET}\right)$ uptake pattern may occur. In this analysis, we investigated the feasibility of ${ }^{18}$ F-FDG PET for response assessment to paclitaxel-carboplatin-bevacizumab (PCB) treatment with and without NTG patches. And we compared the ${ }^{18} \mathrm{~F}-\mathrm{FDG}$ PET response assessment to RECIST response assessment and survival.

\section{Methods}

A total of 223 stage IV non-small cell lung cancer (NSCLC) patients were included in a phase II study (NCT01171170) randomizing between PCB treatment with or without NTG patches. For 60 participating patients, a baseline and a second ${ }^{18} \mathrm{~F}-\mathrm{FDG}$ PET/computed tomography (CT) scan, performed between day 22 and 24 after the start of treatment, were available. Tumor response was defined as a $30 \%$ decrease in CT and PET parameters, and was compared to RECIST response at week 6 . The predictive value of these assessments for progression free survival (PFS) and overall survival (OS) was assessed with and without NTG.

\section{Results}

A $30 \%$ decrease in SUV $_{\text {peak }}$ assessment identified more patients as responders compared to a $30 \%$ decrease in $\mathrm{CT}_{\text {diameter }}$ assessment (73\% vs. $18 \%)$, however, this was not correlated to OS ( $S_{\text {p }}$ peak30 $\mathrm{p}=0.833$; $\left.\mathrm{CT}_{\text {diameter30 }} \mathrm{p}=0.557\right)$. Changes in PET parameters between the baseline and the second scan were not significantly different for the NTG group compared to the control group ( $p$ value range $0.159-0.634$ ). The CT-based (part of the ${ }^{18} \mathrm{~F}-\mathrm{FDG} \mathrm{PET} / \mathrm{CT}$ ) parameters showed a significant difference between the baseline and the second scan for the NTG group compared to the control group ( $\mathrm{CT}_{\text {diameter }}$ decrease of $7 \pm 23 \%$ vs. $19 \pm 14 \%, \mathrm{p}=0.016$, respectively).

\section{Conclusion}

The decrease in tumoral FDG uptake in advanced NSCLC patients treated with chemotherapy with and without NTG did not differ between both treatment arms. Early PET-based response assessment showed more tumor responders than CT-based response assessment (part of the ${ }^{18} \mathrm{~F}-\mathrm{FDG}$ PET/CT); this was not correlated to survival. This might be due to timing of the ${ }^{18} \mathrm{~F}-\mathrm{FDG}$ PET shortly after the bevacizumab infusion. 


\section{Introduction}

Molecular imaging with ${ }^{18} \mathrm{~F}$-fluorodexoyglucose positron emission tomography $\left({ }^{18} \mathrm{~F}\right.$-FDG $\mathrm{PET}$ ) has an established role in the staging of patients with non-small cell lung cancer (NSCLC). In addition, an increasing number of studies have shown that ${ }^{18} \mathrm{~F}-\mathrm{FDG}$ PET is useful for early response assessment in NSCLC patients treated with cytotoxic chemotherapy $[1,2,3,4]$.

Tumor hypoxia is a common phenomenon in lung cancer, and is related to poor prognosis due to treatment resistance $[5,6,7,8,9,10,11]$. Preclinical studies have shown that nitric oxide (NO)-donating drugs increase blood flow and thereby decrease hypoxia [12]. Nitroglycerin (NTG), a vasodilator, is such a drug. By increasing the tumor blood flow, NTG consequently augments antitumor drug delivery and inhibits hypoxia inducible factor (HIF-1 $\alpha$ ) [13]. In preclinical models, administration of low doses of NTG, at least partially, reverses the hypoxia-induced resistance to anticancer drugs [14].

Yasuda et al. [15] showed that the combination of platinum-based chemotherapy and NTG improves overall survival (OS) in patients with stage IIIb/IV NSCLC. However, two recent randomized studies, including the Dutch NVALT12 study, could not confirm these results and no clinical effect was observed by the addition of NTG $[16,17]$.

Negative correlation between perfusion computed tomography (CT) and hypoxia PET on a population basis were also described in literature [18], suggesting that hypoxia is negatively correlated to tumor blood flow. Consequently, if treatment with NTG improved tumor perfusion, this could translate into a change in FDG uptake [13]. This concept was tested in the context of the randomized NVALT12 study that sought to investigate whether the addition of NTG to first-line paclitaxel-carboplatin-bevacizumab (PCB) chemotherapy would improve progression free survival (PFS).

In clinical practice, tumor response assessment is based on changes in tumor size, according to response evaluation criteria in solid tumors (RECIST) at week $6[19,20]$. However, response monitoring is complex because the tumor has to change significantly in size and shape before a response is reliably detected by CT [21, 22]. This leads to an underestimation of the efficacy of cytostatic therapeutic agents that stabilize the disease, in contrast to conventional cytotoxic drugs, which induce shrinkage of tumor dimensions in the case of tumor response [19]. Metabolic changes, measured by ${ }^{18}$ F-FDG PET, will occur earlier than changes in size and may, therefore, be used for early treatment response assessment. A decrease in metabolic activity of the primary tumor after one cycle of chemotherapy treatment is predictive for better outcome $[1,18,23,24]$.

In this paper, we investigated the feasibility of ${ }^{18} \mathrm{~F}$-FDG PET for response assessment to PCB treatment with and without NTG patches. Furthermore, we compared the ${ }^{18} \mathrm{~F}-\mathrm{FDG}$ PET response with both the commonly used RECIST and survival.

\section{Material and Methods}

\section{Patient characteristics}

In the multicentric NVALT12 trial (NCT01171170), 223 patients with metastatic nonsquamous NSCLC were randomized between PCB with or without NTG (see [17] for patient inclusion criteria and treatment specifications) with the primary endpoint of that trial being PFS. Response was assessed every two cycles by the local investigator according to RECIST 1.1 based on CT imaging [20]. In patients undergoing ${ }^{18} \mathrm{~F}-\mathrm{FDG}$ PET/CT at 
baseline as part of the standard work-up (median number of days between baseline scan and start treatment 17 days; range 73 days before treatment to 1 scan performed 1 after the start of treatment), the protocol pre-specified a second ${ }^{18} \mathrm{~F}$-FDG PET/CT between day 22 and 24 (after second chemotherapy infusion and with NTG application for patients in the experimental group; Figure 6.1. To include more patients (17) in the analysis presented here, scans acquired with a time interval between the first chemotherapy and the second ${ }^{18} \mathrm{~F}-\mathrm{FDG}$ PET/CT scan less than 35 days were accepted. This study was approved by the medical ethical committee and all patients provided informed written consent prior to any study handling.

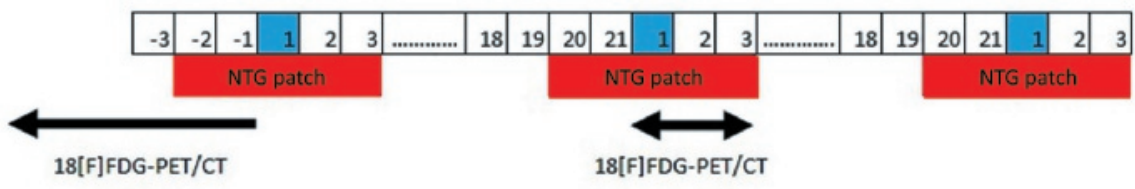

Figure 6.1: NVALT12 trial timeline. At day one of the 21-day cycle, the paclitaxel-carboplatinbevacizumab therapy is administered (grey square). The patients in the experimental arm wear the nitroglycerin (NTG) patch from day -3 to +2 . The baseline ${ }^{18} \mathrm{~F}-\mathrm{FDG}$ PET/CT is performed before the start of chemotherapy and the second ${ }^{18} \mathrm{~F}-\mathrm{FDG} \mathrm{PET} / \mathrm{CT}$ is performed between day 22 and 24 (black arrow). The baseline diagnostic CT is performed before the start of chemotherapy and repeated after every two cycles of chemotherapy (grey arrow).

\section{Scan protocol}

Injected ${ }^{18}$ F-FDG activity depended on individual patient and scanner characteristics, following the Netherlands protocol for standardization of ${ }^{18} \mathrm{~F}$-FDG whole-body PET studies in multi-center trials (NEDPAS) [25], which was the precursor of the EANM guidelines, and images were reconstructed to institutional standards. Typically, a low-dose CT scan as part of the 18F-FDG PET/CT was made, according to institutional standards, and used for attenuation correction. Due to variations between the institutes, for quality control purposes, a spherical volume of interest (VOI) with a diameter of $3 \mathrm{~cm}$ was delineated in the right lobe of the liver [26]. This measurement was used as quality index and scans with a mean standardized uptake value (SUV) of the liver below 1.3 or above 3.0 were excluded from further analysis [27].

\section{Early prediction of survival}

The primary tumor was manually delineated by experienced radiation oncologists using a treatment planning system (Eclipse Version 11.0, Varian Medical Systems, Inc.) and used as the region of interest (ROI). A standard delineation protocol was used, which included fixed window/level settings of CT (lung: 1700/-300; mediastinum: 600/40). Patients without a measurable primary tumor on the baseline ${ }^{18} \mathrm{~F}-\mathrm{FDG}$ PET/CT scan were excluded for analysis.

The maximum standardized uptake value $\left(S U V_{\text {max }}\right)$, mean SUV $\left(S U V_{\text {mean }}\right)$, peak SUV (SUV peak; mean uptake in a sphere with a diameter of $1.2 \mathrm{~cm}$ [21], total lesion 
glycolysis (TLG; TLG was defined by $\mathrm{SUV}_{\text {mean }}$ multiplied by the tumor volume), maximal $\mathrm{CT}$ diameter and $\mathrm{CT}$ volume (number of voxels within the delineated ROI multiplied by the voxel size) were calculated in our institute on the ${ }^{18} \mathrm{~F}-\mathrm{FDG}$ PET/CT scan (Matlab R2013a, The Mathworks, Natrick, MA, USA) using an adapted version of CERR (Computational Environment for Radiotherapy Research) extended with in-house developed Radiomics image analysis software to extract imaging features [28, 29]. Early metabolic response was defined using relative changes in ${ }^{18} \mathrm{~F}-\mathrm{FDG}$ PET uptake parameters of the primary tumor expressed as a percentage change from baseline. Patients were grouped according to a $30 \%$ decrease in CT and PET parameters in the primary tumor ROI of the ${ }^{18} \mathrm{~F}-\mathrm{FDG} \mathrm{PET} / \mathrm{CT}$ scan [26, 30, 31]. For the PET response assessment, SUV peak was used and for the CT response assessment, CT diameter (CT was part of the ${ }^{18} \mathrm{~F}-\mathrm{FDG}$ PET/CT) was used [26, 30]. The RECIST analysis performed during week 6 by the local investigators was used in the analysis to separate patients into responders and non-responders. The $30 \%$ CT and PET response assessments, performed after 3 weeks of therapy, were compared against the RECIST response assessment performed during week 6 by a specificity and sensitivity analysis.

\section{Statistics}

Since normality checks suggested an abnormal distribution for the changes in CT and PET parameters from baseline, non-parametric tests were used for the analysis of these variables. Comparison of the mean changes in CT and PET parameters from baseline for responder vs. non-responders was carried out by an independent samples Mann-Whitney U test. PFS was defined as the interval from randomization to progressive disease or death, whichever occurred first, and OS was defined as the interval from randomization to death from any cause. Differences in PFS and OS were investigated using Cox regression. For calculating the hazard ratio (HR), the different response assessment criteria were used, as a binary variable. To compare CT diameter and SUV $_{\max }$ response with survival, in the waterfall plots a survival cut-off of 6 months was used. This is the median PFS of the combined group (NTG group combined with control group). Statistical tests were based on a two-sided significance level, and the level of significance was set at 0.05 . All statistics were performed in SPSS v.21 (IBM Corp. Released 2012, IBM SPSS Statistics for Windows, Version 21.0, Armonk, NY, USA).

\section{Results}

\section{Patients}

87 out of the 223 included patients in the randomized phase II study had two ${ }^{18} \mathrm{~F}-\mathrm{FDG}$ PET/CT scans available with a measurable primary tumor; however, 27 patients were subsequently excluded for analysis due to poor image quality (see Methods). Hence, 60 patients (characteristics in Table 16.1) had two evaluable consecutive ${ }^{18} \mathrm{~F}-\mathrm{FDG}$ PET/CT scans (Figure 6.2) with a median interval of 42 days. PFS and OS were similar for patients treated with PCB and PCB + NTG (Table 6.1). 


\section{Image characteristics}

\section{Experimental vs. control arm: does NTG leads to decreased FDG uptake}

The mean decrease in SUV $\max$ between the 131 patients treated with PCB (46 $\pm 27 \%)$ and the 29 patients treated with PCB + NTG (42 $\pm 29 \%)$ was not statistically significantly different $(p=0.510)$. The other PET parameters $\left(S V_{\text {mean }}, S_{\text {peak }}\right.$ and TLG) showed on average $<40 \%$ decrease from baseline, but this was also not statistically significantly different between the experimental arm and the control arm (Figure 6.3). Although for CT, part of the ${ }^{18} \mathrm{~F}-\mathrm{FDG}$ PET/CT, in the control arm, the CT diameter decreased significantly more than in the experimental arm (19 $\pm 14 \%$ vs. $7 \pm 23 \%$; $=0.028)$.

\section{Early prediction of survival}

According to the $30 \%$ PET criteria, $74 \%$ of patients in the control arm and $72 \%$ of the patients in the experimental arm showed response after 3 weeks (median time interval 42 days). According to the $30 \%$ CT criteria, $26 \%$ of the patients in the control arm and

\begin{tabular}{|c|c|c|c|}
\hline & & Control & Experimental \\
\hline Patients analyzed & & 31 & 29 \\
\hline \multirow[t]{2}{*}{ Gender } & Male & 15 & 15 \\
\hline & Female & 16 & 14 \\
\hline Age (mean, range) & [years] & $59(39-73)$ & $59(45-77)$ \\
\hline \multirow[t]{3}{*}{ WHO-PS } & 0 & $20(65 \%)$ & $10(34 \%)$ \\
\hline & 1 & $10(32 \%)$ & $18(62 \%)$ \\
\hline & 2 & $1(3 \%)$ & $1(3 \%)$ \\
\hline \multirow[t]{3}{*}{ Smoker } & Current & $13(42 \%)$ & $14(48 \%)$ \\
\hline & Ex & $14(45 \%)$ & $12(41 \%)$ \\
\hline & Never & $4(13 \%)$ & $3(11 \%)$ \\
\hline \multirow[t]{3}{*}{ Histology } & Adeno & $27(86 \%)$ & $24(83 \%)$ \\
\hline & Large cell & $2(7 \%)$ & $1(3 \%)$ \\
\hline & Other & $2(7 \%)$ & $4(14 \%)$ \\
\hline \multirow[t]{2}{*}{ Survival (median, range) } & PFS [months] & $7(3-25)$ & $4(1-11)$ \\
\hline & OS [months] & $13(4-33)$ & $9(2-29)$ \\
\hline \multirow[t]{4}{*}{ RECIST response (week 6) } & Complete response & $0(0 \%)$ & $0(0 \%)$ \\
\hline & Partial response & $9(29 \%)$ & $5(17 \%)$ \\
\hline & Stable disease & $20(65 \%)$ & $17(59 \%)$ \\
\hline & Progressive disease & $2(6 \%)$ & $7(24 \%)$ \\
\hline \multirow[t]{6}{*}{ Baseline (mean, range) } & $\mathrm{CT}$ diameter $[\mathrm{cm}]$ & $6.8(2.5-12.1)$ & $6.7(2.4-16.4)$ \\
\hline & $\mathrm{CT}$ volume $\left[\mathrm{cm}^{3}\right]$ & $101.5(4.4-474.5)$ & $89.8(3.0-468.8)$ \\
\hline & SUVmax & $13.5(3.4-28.9)$ & $14.5(3.6-44.6)$ \\
\hline & SUVmean & $5.7(2.5-11.8)$ & $6.2(2.1-22.3)$ \\
\hline & SUVpeak & $11.0(3.1-25.3)$ & $12.1(2.6-37.9)$ \\
\hline & TLG $\left[S U V \times \mathrm{cm}^{3}\right]$ & $655.0(23.4-4288.8)$ & $638.4(8.2-3467.2)$ \\
\hline
\end{tabular}

WHO-PS World Health Organization performance status, PFS progression free survival, OS overall survival, RECIST response evaluation criteria in solid tumors,

SUV standardized uptake value, TLG total lesion glycolysis.

Table 6.1: Patient characteristics. 


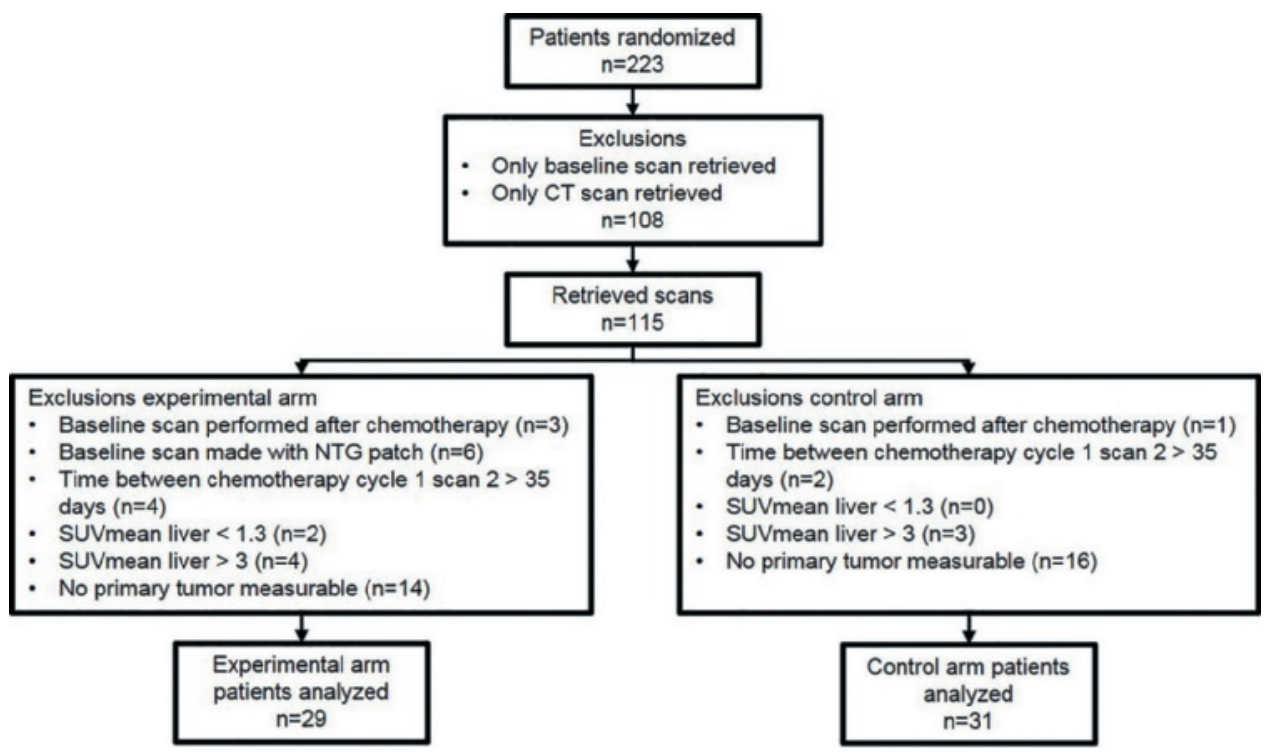

Figure 6.2: CONSORT diagram.

$10 \%$ of the patients in the experimental arm had a response. According to the RECIST analysis performed after 2 cycles (median time interval 56 days) by the local investigator, $29 \%$ of the patients in the control arm had a response and $17 \%$ of the patients in the experimental arm had a response (Table 6.1).

The predictive value of the $30 \%$ CT-based and $30 \%$ PET-based response assessments performed after 3 weeks (on the primary tumor) was assessed for response according to RECIST after 2 cycles (Table 6.2). The $30 \%$ PET-based response assessment had a higher sensitivity compared to the $30 \%$ CT-based response assessment but a lower specificity (Table 6.2).

The $30 \%$ CT-based and $30 \%$ PET-based response assessments were for neither of the arms predictive for PFS nor OS (Table 6.3).

The changes in $C T$ diameter and $\mathrm{SUV}_{\max }$ between baseline and early response assessment were depicted in a waterfall plot showing that PET defined more patients as responders than CT (Figure 6.4 and Table 6.2). However, this decline was not predictive for longer PFS (than 6 months).

\section{Discussion}

The hypothesis of the NVALT 12 trial was that the addition of NTG, by increasing tumor blood flow and oxygenation status, would improve outcome. While the clinical study of the NVALT12 already showed that NTG did improve outcome, in the current study, we investigated of we could predict outcome based on early response assessment using ${ }^{18} \mathrm{~F}$ FDG PET imaging [17]. This image analysis study of the NVALT12 trial could not show a predictive value of ${ }^{18}$ F-FDG PET imaging for the evaluation of the addition of NTG to bevacizumab-containing chemotherapy when compared to control patients. In a previous study, the administration of NO donating drugs decreased hypoxia-induced resistance to 
anticancer drugs in cancer cell lines [14]. In the NVALT12 trial, this could not be confirmed based on ${ }^{18} \mathrm{~F}$-FDG PET analysis. This could be due to a lower NTG dose than that used in the Yasuda study [15], or to an interference with bevacizumab. From recent studies, it is known that FDG is only a moderate surrogate for hypoxia [32]. The study of Zegers et al. [33] showed that $42 \pm 21 \%$ of the primary tumor volume has a high FDG uptake (SUV < $50 \%$ of SUV $\max$ ) of which $10 \pm 12 \%$ is hypoxic (high ${ }^{18} \mathrm{~F}-\mathrm{HX} 4$ uptake TBR $<1.4$ ), and that $3 \%$ of the primary tumor volume outside the high FDG uptake volume is hypoxic as depicted by ${ }^{18} \mathrm{~F}-\mathrm{HX} 4 \mathrm{PET}$. In our study, we, therefore, only measure the effects of NTG on tumor metabolism and survival but not on hypoxia directly. Surprisingly, in nearly all patients, irrespective of treatment arm, a major decrease in FDG uptake was observed in the ${ }^{18}$ F-FDG PET scan performed after 3 weeks. Importantly, this ${ }^{18}$ F-FDG PET scan was acquired within 3 days after the administration of the second cycle of chemotherapy. A study by van der Veldt et al. [34] showed that bevacizumab reduces tumor perfusion and ${ }^{11} \mathrm{C}$-docetaxel uptake in NSCLC, which was accompanied by rapid reduction in circulating levels of VEGF. This decrease in tumor blood flow after bevacizumab administration may explain the lower uptake of FDG in the tumor. Consequently, our results do not exclude the possibility that NTG decreases hypoxia.

\begin{tabular}{l|l|l|l|} 
& RECIST Responder & RECIST non-responder & Total \\
\hline CT diameter decrease $>30 \%$ & $9(15 \%)$ & $2(3 \%)$ & $11(18 \%)$ \\
CT diameter decrease $<30 \%$ & $17(28 \%)$ & $32(54 \%)$ & $49(82 \%)$ \\
& $26(43 \%)$ & $34(57 \%)$ & $60(100 \%)$ \\
SUV peak decrease $>30 \%$ & Sensitivity $=35 \%$ & Specificity $=94 \%$ & \\
SUV peak decrease $<30 \%$ & $23(38 \%)$ & $21(35 \%)$ & $44(73 \%)$ \\
& $3(5 \%)$ & $13(22 \%)$ & $16(27 \%)$ \\
& $26(43 \%)$ & $34(57 \%)$ & $60(100 \%)$ \\
& Sensitivity $=88 \%$ & Specificity $=38 \%$ & \\
\hline
\end{tabular}

RECIST response evaluation criteria in solid tumors, SUV standardized uptake value.

Table 6.2: Comparison of $30 \%$ CT-based and $30 \%$ PET-based response assessment performed after 3 weeks with the RECIST response assessment of week 6 .

\begin{tabular}{|c|c|c|c|c|c|}
\hline \multicolumn{2}{|c|}{ SUV parameter } & \multicolumn{2}{|l|}{ PFS } & \multicolumn{2}{|l|}{ OS } \\
\hline & & HR $(95 \% \mathrm{Cl})$ & $\mathrm{p}$ value & $\mathrm{HR}(95 \% \mathrm{Cl})$ & p-value \\
\hline \multirow{6}{*}{$\begin{array}{l}30 \% \\
\text { response } \\
\text { assessment }\end{array}$} & SUVmax & $1.048(0.591-1.858)$ & 0.874 & $1.025(0.572-1.837)$ & 0.934 \\
\hline & SUVmean & $0.941(0.527-1.680)$ & 0.838 & $0.901(0.501-1.619)$ & 0.726 \\
\hline & SUVpeak & $0.929(0.514-1.679)$ & 0.807 & $0.938(0.515-1.706)$ & 0.833 \\
\hline & TLG & $0.706(0.355-1.406)$ & 0.323 & $1.511(0.722-3.160)$ & 0.273 \\
\hline & CTvolume & $1.073(0.617-1.866)$ & 0.802 & $1.338(0.740-2.419)$ & 0.335 \\
\hline & CTdiameter & $0.718(0.370-1.390)$ & 0.325 & $0.805(0.390-1.662)$ & 0.557 \\
\hline
\end{tabular}

SUV standardized uptake value, TLG total lesion glycolysis, PFS progression free survival, OS overall survival, HR hazard ratio, $\mathrm{Cl}$ confidence interval.

Table 6.3: The hazard ratios (HR) for $30 \%$ PET- and CT-based response assessment with $95 \%$ confidence interval and corresponding $\mathrm{p}$ values for OS and PFS are shown per parameter. 


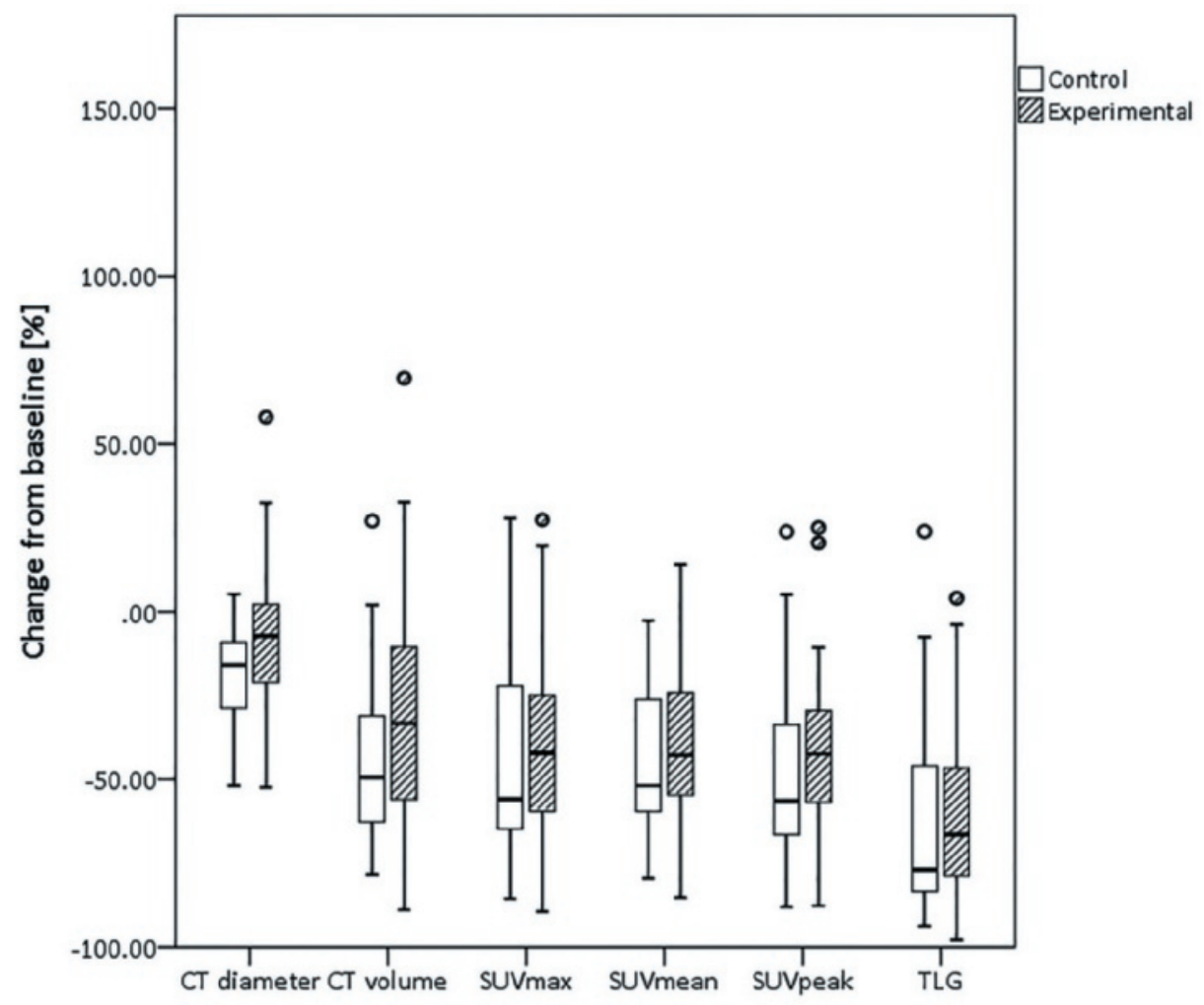

Figure 6.3: Mean values and standard deviations for the CT- and PET-derived image parameters for the experimental arm and the control arm. $p$ values of the independent samples Mann-Whitney $\mathrm{U}$ test of the mean change from baseline of the control arm vs. the mean change from baseline of the experimental arm (*significantly different for the experimental arm compared to the control arm with a significance level of $5 \%$ ). SUV: standardized uptake value; TLG: total lesion glycolysis.

A number of studies have demonstrated that changes in SUV parameters as early as the third week after the start of treatment are predictive for response to chemotherapy and PFS $[1,23,24,35]$. A variety of approaches have been developed to measure the response, starting with the World Health Organization (WHO) criteria and continuing to RECIST and RECIST $1.1[20,26,36]$. These criteria refer to an anatomical decrease in tumor diameter. However, this response must be viewed with some caution when one is trying to predict outcomes in therapies that may be more cytostatic than cytotoxic. With such therapies, lack of progression may be associated with a good improvement in outcome, even in the absence of major shrinkage of tumors [37]. Newer metrics such as PET may be more informative [38]. PET/CT-based response evaluation has proven to be valuable in chemotherapy [39]. Currently, two sets of treatment response criteria for PET are available: EORTC and PET response criteria in solid tumors (PERCIST) [30]. PERCIST operates with a fixed region of interest (ROI) of $1 \mathrm{~cm}^{3}$ in the most ${ }^{18} \mathrm{~F}-\mathrm{FDG}$-avid part of the single most metabolically active tumor in the patient at each PET/CT scan. In the current study, a specific ROI, defining the primary tumor, was used for response evaluation. A consideration for anatomic and functional imaging is that many of the 
changes in response are at the border zones between response groups.
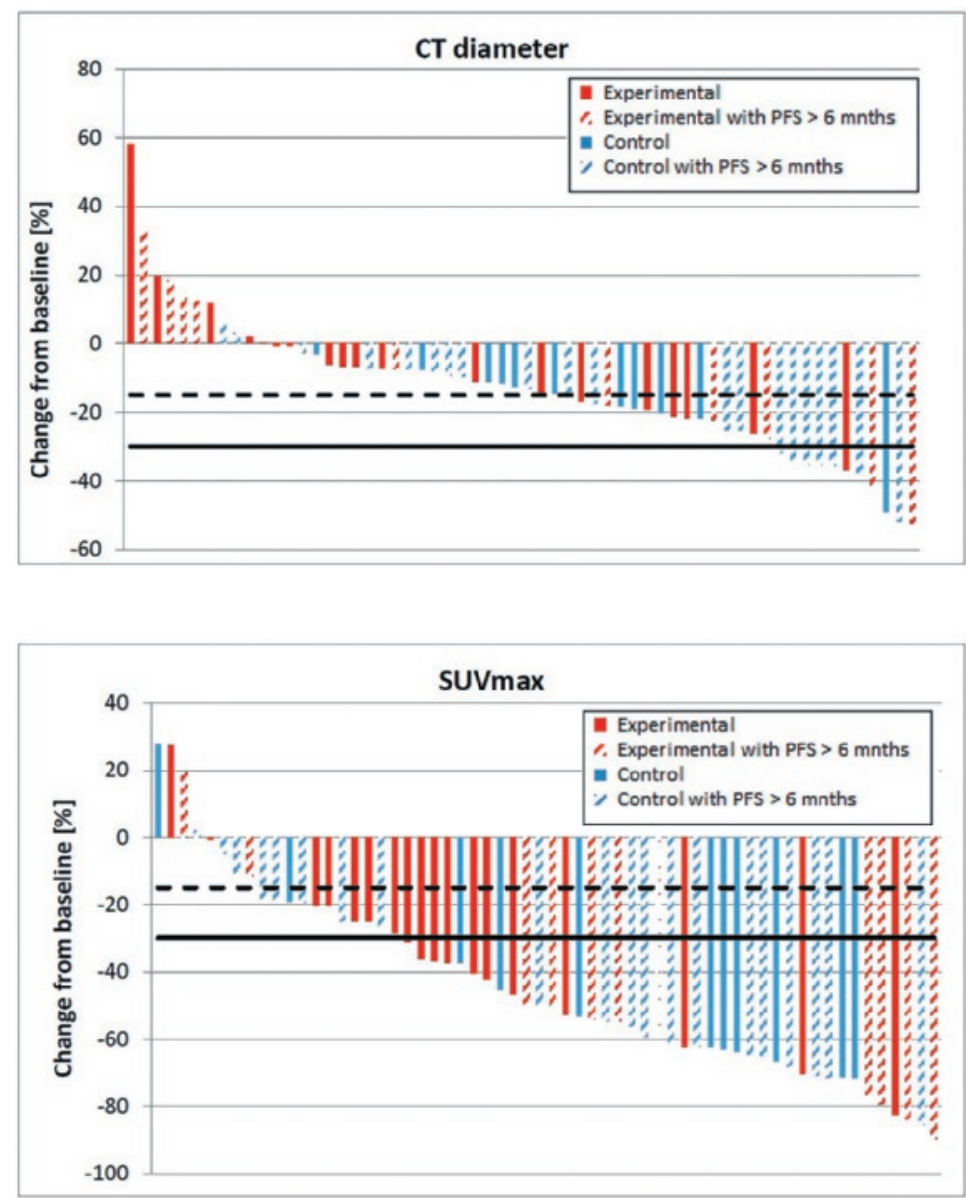

Figure 6.4: Change in CT diameter (upper) and SUVmax (lower) from baseline in individual patients. Patients of the experimental arm are plotted in red, patients of the control arm in blue. The pattern filled bars represent patients with a progression free survival longer than 6 months. The black line represents the used response threshold of $30 \%$. SUV: standardized uptake value; PFS: progression free survival.

These border zones are quite artificial, as changes in tumor size are on a continuous scale (Figure 6.3). The comparison of $30 \%$ CT-based and $30 \%$ PET-based response assessment performed after 3 weeks (median time interval between scans 42 days) with the RECIST analysis performed in week 6 (median time interval between scans 56 days) showed that the RECIST analysis defined more patients as responders than the $30 \%$ CT-based analysis performed after 3 weeks. This can be caused by the difference in timing but also due to the fact that for the $30 \%$ CT-based analysis, only one lesion was measured while in RECIST, multiple lesions were measured. The 30 \% PET-based response assessment performed after 3 weeks showed more responders than the RECIST analysis, 
which is probably caused by decreased perfusion due to the bevacizumab treatment, which led to a decrease in FDG uptake for both treatment arms. The response assessment for PET was not influenced by NTG. A previous study of our group showed that after 3 weeks of treatment, five of nine patients were classified as responders by $C T$ while six of nine were classified as responders by ${ }^{18}$ F-FDG PET [40]. In the same study, patients with a metabolic response (decrease in SUV $<20 \%$ ) at week 3 had a longer PFS than those without (9.7 months vs. 2.8 months), while patients with a response on CT at week 3 did not have a significantly longer PFS than those without. These two findings combined showed that PET may be able to show treatment response earlier than CT. In the former study, ${ }^{18}$ F-FDG PET scans were performed before bevacizumab infusion, while in our study the ${ }^{18} \mathrm{~F}$-FDG PET scan was performed shortly after bevacizumab infusion. This might have impacted the uptake of FDG. A study of Hoekstra et al. [41], also shows that ${ }^{18}$ F-FDG PET has additional value over conventional radiologic techniques for monitoring response in locally advanced NSCLC patients.

The scans used for this study were made within the scope of the Dutch multicenter NVALT12 phase II trial and 60/223 patients underwent $2{ }^{18} \mathrm{~F}-\mathrm{FDG}$ PET scans with the second scan after cycle 2 , but before day 35 . For quality control purposes, only scans with a mean SUV in the liver between 1.3 and 3 were used, reducing the number of assessed patients in this analysis to only 60 of the 223 original patients. For the analysis, these 60 patients were also divided between the control and the experimental arm, which means the study cohort was limited in size, hampering in-depth subgroup analyses.

\section{Conclusion}

The addition of NTG did not lead to enhanced reduction in FDG uptake compared to the control arm. Although PET-based response assessment identified more responders than CT-based response assessment, this did not correlate to progression-free survival or overall survival. This might be due to the timing of the ${ }^{18} \mathrm{~F}$-FDG PET shortly after the bevacizumab infusion. 


\section{References}

[1] L. F. de Geus-Oei et al. "Chemotherapy response evaluation with 18F-FDG PET in patients with non-small cell lung cancer". In: J Nucl Med 48.10 (2007). Journal of nuclear medicine : official publication, Society of Nuclear Medicine, pp. 1592-8. DOI: 10.2967/jnumed.107.043414. URL: http://www.ncbi.nlm.nih.gov/pubmed/ 17873138.

[2] M. Vaidya et al. "Combined PET/CT image characteristics for radiotherapy tumor response in lung cancer". In: Radiother Oncol 102.2 (2012). Radiotherapy and oncology: journal of the European Society for Therapeutic Radiology and Oncology, pp. 239-45. DOI: 10.1016/j.radonc.2011.10.014.

[3] C. Ordu et al. "Does early PET/CT assesment of response to chemotherapy predicts survival in patients with advanced stage non-small-cell lung cancer?" In: Medicine (Baltimore) 93.28 (2014). Medicine, e299. DOI: 10.1097/md.0000000000000299.

[4] W. Grootjans et al. "PET in the management of locally advanced and metastatic NSCLC". In: Nat Rev Clin Oncol 12.7 (2015). Nature reviews. Clinical oncology, pp. 395-407. DOI: $10.1038 /$ nrclinonc.2015.75.

[5] M. Hockel and P. Vaupel. "Tumor hypoxia: definitions and current clinical, biologic, and molecular aspects". In: J Natl Cancer Inst 93.4 (2001). Journal of the National Cancer Institute, pp. 266-76.

[6] A. L. Harris. "Hypoxia-a key regulatory factor in tumour growth". In: Nat Rev Cancer 2.1 (2002). Nature reviews. Cancer, pp. 38-47. DOI: 10.1038/nrc704.

[7] E. E. Verwer, R. Boellaard, and A. A. van der Veldt. "Positron emission tomography to assess hypoxia and perfusion in lung cancer". In: World J Clin Oncol 5.5 (2014). World journal of clinical oncology, pp. 824-44. DOI: 10.5306/wjco.v5.i5.824. URL: http://www.ncbi.nlm.nih.gov/pubmed/25493221.

[8] B. G. Wouters et al. "Hypoxia as a target for combined modality treatments". In: Eur J Cancer 38.2 (2002). European journal of cancer (Oxford, England : 1990), pp. 240-57.

[9] B. G. Wouters et al. "Targeting hypoxia tolerance in cancer". In: Drug Resist Updat 7.1 (2004). Drug resistance updates : reviews and commentaries in antimicrobial and anticancer chemotherapy, pp. 25-40. DOI: 10.1016/j.drup.2003.12.004.

[10] L. J. Dubois et al. "New ways to image and target tumour hypoxia and its molecular responses". In: Radiother Oncol 116.3 (2015). Radiotherapy and oncology : journal of the European Society for Therapeutic Radiology and Oncology, pp. 352-7. DOI: 10.1016/j.radonc. 2015.08.022.

[11] S. G. Peeters et al. "Current preclinical and clinical applications of hypoxia PET imaging using 2-nitroimidazoles". In: Q J Nucl Med Mol Imaging 59.1 (2015). The quarterly journal of nuclear medicine and molecular imaging : official publication of the Italian Association of Nuclear Medicine (AIMN) [and] the International Association of Radiopharmacology (IAR), [and] Section of the Society of..., pp. 39-57. 
[12] B. F. Jordan et al. "Changes in tumor oxygenation/perfusion induced by the no donor, isosorbide dinitrate, in comparison with carbogen: monitoring by EPR and MRI". In: Int J Radiat Oncol Biol Phys 48.2 (2000). International journal of radiation oncology, biology, physics, pp. 565-70.

[13] H. Yasuda et al. "Nitroglycerin treatment may enhance chemosensitivity to docetaxel and carboplatin in patients with lung adenocarcinoma". In: Clin Cancer Res 12.22 (2006). Clinical cancer research : an official journal of the American Association for Cancer Research, pp. 6748-57. DOI: 10.1158/1078-0432.ccr-06-1124.

[14] N. E. Matthews et al. "Nitric oxide-mediated regulation of chemosensitivity in cancer cells". In: J Natl Cancer Inst 93.24 (2001). Journal of the National Cancer Institute, pp. 1879-85.

[15] H. Yasuda et al. "Randomized phase II trial comparing nitroglycerin plus vinorelbine and cisplatin with vinorelbine and cisplatin alone in previously untreated stage IIIB/IV non-small-cell lung cancer". In: J Clin Oncol 24.4 (2006). Journal of clinical oncology : official journal of the American Society of Clinical Oncology, pp. 688-94. DOI: 10.1200/jco.2005.04.0436.

[16] A. Davidson et al. "A phase III randomized trial of adding topical nitroglycerin to first-line chemotherapy for advanced nonsmall-cell lung cancer: the Australasian lung cancer trials group NITRO trial". In: Ann Oncol 26.11 (2015). Annals of oncology : official journal of the European Society for Medical Oncology, pp. 2280-6. DOI: 10.1093/annonc/mdv373.

[17] A. M. Dingemans et al. "A randomized phase II study comparing paclitaxel-carboplatin-bevacizumab with or without nitroglycerin patches in patients with stage IV nonsquamous nonsmall-cell lung cancer: NVALT12 (NCT01171170)". In: Ann Oncol 26.11 (2015). Annals of oncology : official journal of the European Society for Medical Oncology, pp. 2286-93. DOI: $10.1093 /$ annonc/mdv370.

[18] W. van Elmpt et al. "Multiparametric imaging of patient and tumour heterogeneity in non-small-cell lung cancer: quantification of tumour hypoxia, metabolism and perfusion". In: Eur J Nucl Med Mol Imaging 43.2 (2016). European journal of nuclear medicine and molecular imaging, pp. 240-8. DOI: 10.1007/s00259-015-3169-4.

[19] M. Hachemi et al. "[(1)(8)F]FDG positron emission tomography within two weeks of starting erlotinib therapy can predict response in non-small cell lung cancer patients". In: PLoS One 9.2 (2014). PloS one, e87629. DOI: 10.1371/journal.pone.0087629.

[20] E. A. Eisenhauer et al. "New response evaluation criteria in solid tumours: revised RECIST guideline (version 1.1)". In: Eur J Cancer 45.2 (2009). European journal of cancer (Oxford, England: 1990), pp. 228-47. DOI: 10.1016/j.ejca.2008.10.026.

[21] H. Baghaei et al. "Principles of Positron Emission Tomography Imaging". In: Clinical PET and PET/CT. New York: Springer, 2013. DOI: https://doi.org/10.1007/9781-4419-0802-5_1.

[22] J. J. Erasmus et al. "Interobserver and intraobserver variability in measurement of non-small-cell carcinoma lung lesions: implications for assessment of tumor response". In: J Clin Oncol 21.13 (2003). Journal of clinical oncology : official journal of the American Society of Clinical Oncology, pp. 2574-82. DOI: 10.1200/jco.2003.01.144. 
[23] D. Kahraman et al. "Quantitative analysis of response to treatment with erlotinib in advanced non-small cell lung cancer using 18F-FDG and 3'-deoxy-3'-18F-fluorothymidine PET". In: J Nucl Med 52.12 (2011). Journal of nuclear medicine : official publication, Society of Nuclear Medicine, pp. 1871-7. DOI: $10.2967 /$ jnumed.111.094458.

[24] C. Nahmias et al. "Time course of early response to chemotherapy in non-small cell lung cancer patients with 18F-FDG PET/CT". In: J Nucl Med 48.5 (2007). Journal of nuclear medicine : official publication, Society of Nuclear Medicine, pp. 744-51. DOI: $10.2967 /$ jnumed.106.038513.

[25] R. Boellaard et al. "The Netherlands protocol for standardisation and quantification of FDG whole body PET studies in multi-centre trials". In: Eur J Nucl Med Mol Imaging 35.12 (2008). European journal of nuclear medicine and molecular imaging, pp. 2320-33. DOI: 10.1007/s00259-008-0874-2. URL: http://www.ncbi.nlm.nih. gov/pubmed/18704407.

[26] R. L. Wahl et al. "From RECIST to PERCIST: Evolving Considerations for PET response criteria in solid tumors". In: J Nucl Med 50 Suppl 1 (2009). Journal of nuclear medicine : official publication, Society of Nuclear Medicine, 122S-50S. DOI: 10.2967 / jnumed . 108.057307. URL: http: / / www . ncbi .nlm. nih. gov / pubmed/ 19403881.

[27] R. Boellaard et al. "FDG PET/CT: EANM procedure guidelines for tumour imaging: version 2.0". In: Eur J Nucl Med Mol Imaging 42.2 (2015). European journal of nuclear medicine and molecular imaging, pp. 328-54. DOI: 10.1007/s00259-0142961-x. URL: http://www.ncbi.nlm.nih.gov/pubmed/25452219.

[28] J. O. Deasy, A. I. Blanco, and V. H. Clark. "CERR: a computational environment for radiotherapy research". In: Med Phys 30.5 (2003). Medical physics, pp. 979-85. DOI: 10.1118/1.1568978.

[29] C. Parmar et al. "Robust Radiomics feature quantification using semiautomatic volumetric segmentation." eng. In: PloS one 9 (7 2014), e102107.

[30] K. Skougaard et al. "Comparison of EORTC criteria and PERCIST for PET/CT response evaluation of patients with metastatic colorectal cancer treated with irinotecan and cetuximab". In: J Nucl Med 54.7 (2013). Journal of nuclear medicine : official publication, Society of Nuclear Medicine, pp. 1026-31. DOI: 10.2967/jnumed.112.111757.

[31] H. Young et al. "Measurement of clinical and subclinical tumour response using [18F]-fluorodeoxyglucose and positron emission tomography: review and 1999 EORTC recommendations. European Organization for Research and Treatment of Cancer (EORTC) PET Study Group." eng. In: European journal of cancer (Oxford, England : 1990) 35 (13 1999), pp. 1773-82.

[32] S. G. Peeters et al. "A comparative study of the hypoxia PET tracers [(1)(8)F]HX4, $[(1)(8) F] F A Z A$, and [(1)(8)F]FMISO in a preclinical tumor model". In: Int J Radiat Oncol Biol Phys 91.2 (2015). International journal of radiation oncology, biology, physics, pp. 351-9. DOI: 10.1016/j.ijrobp.2014.09.045.

[33] C. M. Zegers et al. "In vivo quantification of hypoxic and metabolic status of NSCLC tumors using [18F]HX4 and [18F]FDG-PET/CT imaging". In: Clin Cancer Res 20.24 (2014). Clinical cancer research : an official journal of the American Association for Cancer Research, pp. 6389-97. DOI: 10.1158/1078-0432.ccr-14-1524. 
[34] A. A. van der Veldt, E. F. Smit, and A. A. Lammertsma. "Positron Emission Tomography as a Method for Measuring Drug Delivery to Tumors in vivo: The Example of [(11)C]docetaxel". In: Front Oncol 3 (2013). Frontiers in oncology, p. 208. DOI: $10.3389 /$ fonc. 2013.00208 .

[35] W. van Elmpt et al. "Response assessment using 18F-FDG PET early in the course of radiotherapy correlates with survival in advanced-stage non-small cell lung cancer". In: J Nucl Med 53.10 (2012). Journal of nuclear medicine : official publication, Society of Nuclear Medicine, pp. 1514-20. DOI: 10.2967/jnumed.111.102566.

[36] World Health Organization. WHO handbook for reporting results of cancer treatment. Geneva: World Health Organization, 1979.

[37] G. L. Rosner, W. Stadler, and M. J. Ratain. "Randomized discontinuation design: application to cytostatic antineoplastic agents". In: J Clin Oncol 20.22 (2002). Journal of clinical oncology : official journal of the American Society of Clinical Oncology, pp. 4478-84. DOI: 10.1200/jco.2002.11.126.

[38] L. C. Michaelis and M. J. Ratain. "Measuring response in a post-RECIST world: from black and white to shades of grey". In: Nat Rev Cancer 6.5 (2006). Nature reviews. Cancer, pp. 409-14. DOI: 10.1038/nrc1883.

[39] I. M. Desar et al. "Beyond RECIST: molecular and functional imaging techniques for evaluation of response to targeted therapy". In: Cancer Treat Rev 35.4 (2009). Cancer treatment reviews, pp. 309-21. DOI: 10.1016/j.ctrv.2008.12.001.

[40] A. M. Dingemans et al. "First-line erlotinib and bevacizumab in patients with locally advanced and/or metastatic non-small-cell lung cancer: a phase II study including molecular imaging". In: Ann Oncol 22.3 (2011). Annals of oncology : official journal of the European Society for Medical Oncology / ESMO, pp. 559-66. DOI: 10.1093/ annonc/mdq391. URL: http://www.ncbi.nlm.nih.gov/pubmed/20702788.

[41] C. J. Hoekstra et al. "Prognostic relevance of response evaluation using [18F]-2-fluoro-2-deoxy-D-glucose positron emission tomography in patients with locally advanced non-small-cell lung cancer". In: J Clin Oncol 23.33 (2005). Journal of clinical oncology : official journal of the American Society of Clinical Oncology, pp. 8362-70. DOI: 10.1200/jco.2005.01.1189. 



\section{Chapter 7}

\section{Applicability of a prognostic CT-based radiomic signature model trained on stage I-III non-small cell lung cancer in stage IV non-small cell lung cancer}

Evelyn E.C. de Jong, Wouter van Elmpt, Stefania Rizzo, Anna Colarieti, Gianluca Spitaleri, Ralph T.H. Leijenaar, Arthur Jochems, Lizza E.L. Hendriks, Esther G.C. Troost, Bart Reymen, Anne-Marie C. Dingemans, Philippe Lambin 


\section{Abstract}

\section{Objectives}

Recently it has been shown that radiomic features of computed tomography (CT) have prognostic information in stage I-III non-small cell lung cancer (NSCLC) patients. We aim to validate this prognostic radiomic signature in stage IV adenocarcinoma patients undergoing chemotherapy.

\section{Materials and Methods}

Two datasets of chemo-naive stage IV adenocarcinoma patients were investigated, dataset 1: 285 patients with CTs performed in a single center; dataset 2: 223 patients included in a multicenter clinical trial. The main exclusion criteria were EGFR mutation or unknown mutation status and non-delineated primary tumor. Radiomic features were calculated for the primary tumor. The c-index of cox regression was calculated and compared to the signature performance for overall survival (OS).

\section{Results}

In total CT scans from 195 patients were eligible for analysis. Patients having a Prognostic Index (PI) lower than the signature median $(n=92)$ had a significantly better OS than patients with a PI higher than the median $(\mathrm{n}=103, \mathrm{HR} 1.445,95 \% \mathrm{Cl} 1.07-1.95, \mathrm{p}=0.02$, c-index $0.576,95 \% \mathrm{Cl}$ $0.527-0.624)$.

\section{Conclusion}

The radiomic signature, derived from daily practice $\mathrm{CT}$ scans, has prognostic value for stage IV NSCLC, however the signature performs less than previously described for stage I-III NSCLC stages. In the future, machine learning techniques can potentially lead to a better prognostic imaging based model for stage IV NSCLC. 


\section{Introduction}

Medical imaging, such as computed tomography (CT) and positron emission tomography (PET), is used in standard staging and response assessment procedures in patients with non-small cell lung cancer (NSCLC). In contrast to pathological investigations, medical imaging is non-invasive and able to capture the entire tumor volume at multiple timepoints prior to and during treatment [1]. Recent studies have shown that quantitative imaging features, extracted from routine medical imaging, have prognostic value and may predict clinical outcomes or allow for treatment monitoring in different cancer types $[2,3$, $4,5,6,7]$.

Radiomics is a high-throughput approach to translate medical images into mineable data by extracting a large number of quantitative features describing tumor intensity, shape and texture $[8,9,10]$. A comprehensive and robust quantification of imaging phenotypes provides complementary and clinically relevant information, which may lead to imaging biomarkers $[11,12,13,14,15]$.

In 2014, our group published a prognostic radiomic signature for overall survival (OS), consisting of 4 radiomic features (further referred to as "the signature"), which is exclusively based on pre-treatment CT imaging of the primary tumor volume [16]. The signature consists of the following four features, derived from the primary tumor: 1) "First order statistics: Energy", describing the overall density of the tumor volume; 2) "Shape: Compactness", quantifying the compactness of the tumor volume relative to that of a sphere (i.e. the most compact shape); 3) "Gray level run length: Gray level non-uniformity", a measure of intra-tumor heterogeneity; and 4) Wavelet (HLH) "Gray level run length: Gray level non-uniformity", also describing intra-tumor heterogeneity, but now after wavelet decomposition of the original CT image [17]. A detailed mathematical description of the aforementioned features can be found in Aerts et al. [16]. This signature was derived from stage I-III NSCLC patients treated with radio(chemo)therapy and independently validated in both resected NSCLC (concordance (c-) index 0.65), and stage I-IV head and neck squamous cell carcinoma (HNSCC) patients (c-index 0.69). Next to that, this signature was validated in an oropharyngeal squamous cell carcinoma cohort (c-index 0.63) [17].

Known prognostic clinical variables in stage IV NSCLC are WHO performance status and cM-stage according to the 7th or 8th edition of the TNM staging in NSCLC [18]. Our group showed before that next to lower cM-stage also younger age, female gender, and low cTN-stage are favorable factors for OS in stage IV [19].

In this paper, we aimed to investigate the prognostic value of the signature in two large independent cohorts of stage IV NSCLC patients, EGFR and ALK wildtype, and compared this with prognostic value of clinical variables.

\section{Material and Methods}

\section{Patient characteristics}

The first cohort consisted of 285 patients with incurable advanced stage non-squamous NSCLC tested for epidermal growth factor receptor (EGFR) mutation/Kirsten rat sarcoma (KRAS) mutation/anaplastic lymphoma kinase (ALK) translocation (see Rizzo et al. [20] for patient inclusion criteria and treatment specifications). Patients were treated with cisplatin or carboplatin in combination with pemetrexed. Written informed consent to 
the use of clinical and imaging data for scientific purposes was obtained from all patients beforehand. This study was also approved by the local Ethical Committee (R 422/16).

The second cohort is a dataset of 223 non-squamous NSCLC patients, who were included in a multicenter phase II trial (NVALT12; NCT01171170). In the NVALT12 trial patients were randomized between paclitaxel-carboplatin-bevacizumab with or without nitroglycerin patches (see Dingemans et al. [21] for patient inclusion criteria and treatment specifications). This study was approved by the medical ethical committee, and all patients provided informed written consent prior to any study handling.

Eligiblity criteria were: stage IV non-squamous NSCLC, WHO-PS $<3$, ALK and EGFR wildtype (mutation status is only tested in advanced stage NSCLC). Next to that, patients with a CT-scan without a definable primary tumor, a too small field-of-view, an original tumor volume that was separated by the resampling, or a non-standard patient position were excluded.

\section{Imaging}

For the first cohort all CT scans were performed, before treatment initiation, in the same hospital using a 16-slice Lightspeed GE CT scanner or a 64-slice MSTC Optima 660 GE CT scanner. Diagnostic CT scans with or without contrast were performed. A standard thorax protocol was used with a mean slice thickness of $3 \mathrm{~mm}$. The primary tumor was manually delineated by EJ and checked by an experienced radiation oncologist (BR) using Mirada (Mirada Medical, Oxford, UK) and used as the region of interest (ROI).

For the second cohort CT scans were performed in 16 different hospitals and centrally collected for analysis. Eleven different CT scanners (Siemens, Philips, GE and Toshiba) and seven different PET/CT scanners (Siemens, Philips and GE) were used. Low-dose $\mathrm{CT}$ scans as well as diagnostic CT scans, with and without contrast, were performed. There was no specific CT protocol used, although all CT scans were used for response evaluation criteria in solid tumors (RECIST) 1.1 response assessment, which recommends a slice thickness of $10 \mathrm{~mm}$ or less and for spiral CT a slice thickness of $5 \mathrm{~mm}$, therefore the mean slice thickness was $3 \mathrm{~mm}$. The primary tumor was manually delineated by EJ and checked by an experienced radiation oncologist (ET) and a pulmonologist (LH) using a radiation treatment planning system (Eclipse Version 11.0, Varian Medical Systems Inc., Palo Alto, CA) and used as the ROI. A standard delineation protocol was used, which included fixed window/level settings (lung: 1700/-300; mediastinum: 600/40).

\section{Radiomics}

Image features were calculated on the baseline CT scan using an adapted version of Computational Environment for Radiotherapy Research (CERR) extended with in-house developed radiomic image analysis software (Matlab R2014a, The Mathworks, Natrick, MA) to extract the image features $[14,22]$. A bin width of 25 Hounsfield Units was used. To minimize the possible effect of the variation in image parameters, all scans were resampled to a voxel size of $1 \times 1 \times 3 \mathrm{~mm}^{3}$ (same voxel size as in "the signature" dataset) using a cubic interpolation as recommended in Larue et al. [23].

The signature was based on a Cox proportional hazards model with the following feature coefficients $(\beta): 1$ ) "First order statistics: Energy": $2.42 \times 10^{-11}$; 2) "Shape: Compactness": $\left.-5.38 \times 10^{-3} ; 3\right)$ "Gray level run length: Gray level non-uniformity": -1.47 $\times 10^{-4}$; 4) Wavelet $(\mathrm{HLH})$ "Gray level run length: Gray level non-uniformity": $9.39 \times$ 
$10^{-6}$. The prognostic index $(\mathrm{PI})$ for the radiomic signature, to be used for validation, is then defined as: $\mathrm{PI}=\sum_{i} \beta_{i} x_{i}[17]$.

\section{Clinical variables}

We tested the prognostic value of the clinical variables age, gender, cT-stage, cN-stage, cM-stage (restaged according to the $8^{\text {th }}$ edition of the TNM classification for lung cancer) [18] and the WHO performance status.

\section{Statistics}

The performance of the signature in the cohort of stage IV NSCLC patients was investigated by calculating the c-index using RStudio (Rstudio, Inc., Boston, MA, US) and compared to the c-indices found by Aerts et al. [16].

The prognostic value of the clinical variables was investigated using a univariable and multivariable cox regression in SPSS (IBM SPSS Statistics for Windows, Version 24.0, Armonk, NY, US). A log-rank test was applied to test for significant differences between survival curves. For dataset 1 , OS was defined as the interval from start of treatment for stage IV disease to death from any cause, and from randomization to death from any cause for dataset 2. Patients without event or lost to follow-up were censored at last date known to be alive. Statistical tests were based on a two-sided significance level, and the level of significance was set at $p<0.05$.

\section{Results}

For 261 out of 285 patients of the first cohort a baseline CT scan could be retrieved. Since 203 patients were ineligible (10 patients were excluded for analysis due to the fact that the primary tumor was indefinable, 1 patient due to an incorrect patient position, 1 patient due to a too small field-of-view of the CT scan, 1 patient due to the resampling that caused a separation of the original volume, 14 patients because of WHO-PS score higher than 2, 67 patients had cM0-stage and 109 patients had an EGFR mutation, ALK rearrangement or unknown mutation status (see Figure 7.1 for the CONSORT diagram), a total of 58 scans was eligible for analysis. For the second cohort, baseline CT scans were available for 210 of the 223 patients. 71 patients were ineligible (28 patients due to a non-measurable primary tumor, 1 patient due to an incorrect patient position, 1 patient due to a too small field of view of the CT scan, 2 patients due to the resampling that caused a separation of the original volume, 1 patient due to missing survival data, 6 patients due to a M-stage of zero, 5 patients with an EGFR mutation and 29 patients with an unknown mutation status (see Figure 7.1 for the CONSORT diagram), leaving 137 CT scans suitable for analysis.

In Table 7.1, the patient characteristics of cohort 1 , cohort 2 and the combined cohort can be found. The median follow-up of cohort 1 was 52 months and at time of analysis 3 patients were censored for OS, for cohort 2 the median tumor volume was 28 months and at the time of analysis 14 patients were censored for OS. The median tumor volume of the first cohort and second cohort were comparable (median $36 \mathrm{cc}$, range 2-507 cc vs. median $36 \mathrm{cc}$, range 1-769 cc). The median OS of the cohorts were comparable, 9 months (range 0-65 months) and 10 months (range 0-34 months), respectively.

Due to the relatively small number of eligible CT scans, both cohorts were combined for the further analyses. The PI of the signature on this stage IV dataset had a c-index 
COHORT 1

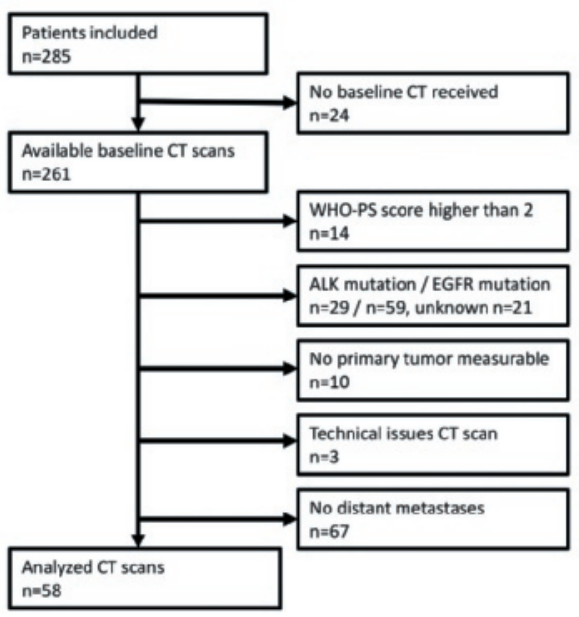

COHORT 2

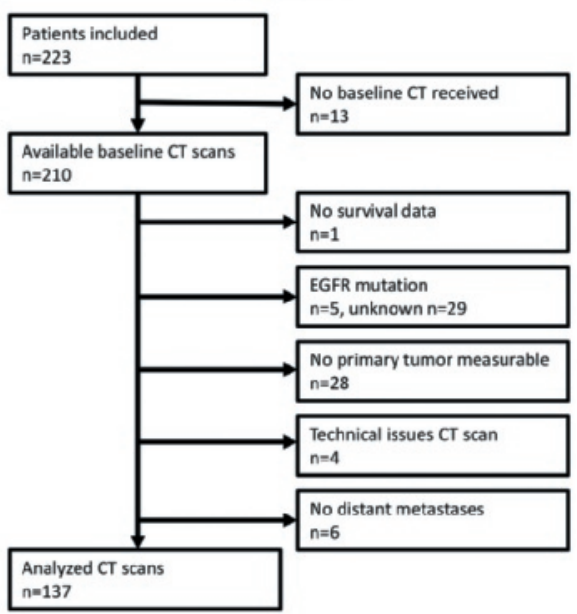

Figure 7.1: CONSORT diagram of cohort 1 (left) and cohort 2 (right).

of $0.576(95 \% \mathrm{Cl} 0.527-0.624, \mathrm{p}=0.002)$. Patients with a PI lower than the median of the signature cohort $(n=92)$ had a significant better OS than patients with a PI higher than the median $(n=103)$; Hazard ratio $1.445(1.07-1.92, p=0.02) ; 13$ vs. 8 months, respectively (Figure 7.2 ).

In the univariable analysis, patients with WHO-PS 1 had a worse prognosis compared to those with WHO-PS 0 ( $n=101$ vs. $n=69$; HR 1.52, $95 \% \mathrm{Cl} 1.10-2.11, p=0.012$ ), patients with cN3 disease had a worse prognosis compared to cN0 patients ( $\mathrm{n}=76$ vs. $\mathrm{n}=28$; HR 1.84, $95 \% \mathrm{Cl} 1.15-2.93, \mathrm{p}=0.01$ ), and those with $\mathrm{cM} 1 \mathrm{c}$ had a worse prognosis compared to patients with cM1a disease ( $\mathrm{n}=62$ vs. $\mathrm{n}=75 ; \mathrm{HR} 1.53,95 \% \mathrm{Cl} 1.07-2.19$, $\mathrm{p}=0.02$; see Table 7.2). The PI was as well prognostic for OS in the univariable analysis (HR 1.46, $95 \% \mathrm{Cl}$ 1.02-2.10, $\mathrm{p}=0.04$ ). In the multivariable analysis, the latter three tumor characteristics retained their prognostic value showing (cN3 vs. cN0; HR 1.92, $95 \% \mathrm{Cl}$ 1.18-3.12, $\mathrm{p}=0.01$; $\mathrm{cM} 1 \mathrm{c}$ vs. $\mathrm{cM} 1 \mathrm{a} ; \mathrm{HR} 1.62,95 \% \mathrm{Cl} 1.11-2.36, \mathrm{p}=0.01$; and PI HR 1.56, $95 \% \mathrm{Cl}$ 1.04-2.33, $\mathrm{p}=0.03$; see Table 7.3). In Supplementary Material Table 7.4 the multivariable analysis excluding the $\mathrm{PI}$ can be found.

\section{Discussion}

We investigated a previous published prognostic radiomic signature consisting of four quantitative image features calculated on baseline CT scans of the primary tumor. This signature has prognostic value in patients with stage I-III NSCLC and HNSCC. In our study we validated this signature in an independent cohort of 195 patients with metastatic (stage IV), EGFR and ALK wildtype, NSCLC.

Aerts et al. [16] found that the signature had a good performance in an independent validation cohort of stage I-III NSCLC patients (c-index 0.65). The signature showed in addition prognostic value in our cohort of stage IV NSCLC, although the c-index was less 
prominent (c-index 0.58, $\mathrm{p}=0.002$ ). However, our cohort of patients was well characterized and in daily practice patients are viewed to have the same prognosis (stage IV, EGFR and ALK wildtype, non-squamous histology, WHO PS $<3$, chemotherapy eligible), by applying the signature and using a routine baseline $\mathrm{CT}$ scan we were able to select a group of patients with poor outcome [HR 1.45 (1.07-1.92), $\mathrm{p}=0.02]$. A reason for the less prominent c-index in our cohort can be that the data from cohort 2 was part of a multicenter study. Although, the CT scans of the different cohorts were delineated using different software packages, no differences were expected in the radiomic features due to the different delineation packages. However, there was no dedicated CT image protocol specified as image specific endpoint, therefore there was quite some variation in the image parameters. Recent publications have shown that resampling of the voxel size leads to more reproducible CT features [24, 25]. Shafiq-Ul-Hassan et al. [24] showed that some radiomic features were even voxel size dependent. To minimize the possible effect of the variation in image parameters, all scans were resampled, as recommended in Larue et al. [23], to a voxel size of $1 \times 1 \times$ $3 \mathrm{~mm}^{3}$, which is comparable to the voxel size of the signature dataset. Therefore the median to split the cohort of patients as used in the signature cohort could be used as well in our cohort of stage IV patients. Therefore in the future we would recommend to perform resampling as recommended in Larue et al. [23] and for validation studies to use

\begin{tabular}{|c|c|c|c|c|}
\hline Variable & & $\begin{array}{l}\text { Cohort 1; } \\
n=58(\%)\end{array}$ & $\begin{array}{l}\text { Cohort 2; } \\
n=137(\%)\end{array}$ & $\begin{array}{l}\text { Combined; } \\
n=195(\%)\end{array}$ \\
\hline \multirow[t]{2}{*}{ Gender } & Male & $37(64 \%)$ & $72(53 \%)$ & $109(56 \%)$ \\
\hline & Female & $21(36 \%)$ & $65(47 \%)$ & $86(44 \%)$ \\
\hline \multirow[t]{3}{*}{ WHO-PS } & 0 & $8(14 \%)$ & $61(45 \%)$ & $69(35 \%)$ \\
\hline & 1 & $31(53 \%)$ & $70(51 \%)$ & $101(52 \%)$ \\
\hline & 2 & $19(33 \%)$ & $6(4 \%)$ & $25(13 \%)$ \\
\hline \multirow[t]{5}{*}{ cT-stage } & 1 & $5(9 \%)$ & $27(20 \%)$ & $32(16 \%)$ \\
\hline & 2 & $17(29 \%)$ & $36(26 \%)$ & $53(27 \%)$ \\
\hline & 3 & $8(14 \%)$ & $26(19 \%)$ & $34(17 \%)$ \\
\hline & 4 & $26(45 \%)$ & $38(28 \%)$ & $64(33 \%)$ \\
\hline & $x$ & $2(3 \%)$ & $10(7 \%)$ & $12(6 \%)$ \\
\hline \multirow[t]{5}{*}{ cN-stage } & 0 & $8(14 \%)$ & $20(15 \%)$ & $28(14 \%)$ \\
\hline & 1 & $2(3 \%)$ & $8(6 \%)$ & $10(5 \%)$ \\
\hline & 2 & $28(48 \%)$ & $43(31 \%)$ & $71(36 \%)$ \\
\hline & 3 & $18(31 \%)$ & $58(42 \%)$ & $76(39 \%)$ \\
\hline & $x$ & $2(3 \%)$ & $8(6 \%)$ & $10(5 \%)$ \\
\hline \multirow[t]{3}{*}{ cM-stage } & $1 \mathrm{a}$ & $10(17 \%)$ & $65(47 \%)$ & $75(38 \%)$ \\
\hline & $1 b$ & $13(22 \%)$ & $45(33 \%)$ & $58(30 \%)$ \\
\hline & 1c & $35(61 \%)$ & $27(20 \%)$ & $62(32 \%)$ \\
\hline \multirow[t]{2}{*}{ Mutation status } & wt & $28(48 \%)$ & $83(61 \%)$ & $111(57 \%)$ \\
\hline & KRAS & $30(52 \%)$ & $54(39 \%)$ & $84(43 \%)$ \\
\hline Median OS [months] & & $9(0-65)$ & $10(0-34)$ & $10(0-65)$ \\
\hline Median Tumor volume [cc] & & $36(2-507)$ & $36(1-769)$ & $36(1-769)$ \\
\hline
\end{tabular}

PS performance status, cT clinical Tumor-stage, cN clinical Nodal-stage, cM clinical Metastases-stage, wt wildtype, KRAS Kirsten rat sarcoma,

OS overall survival.

Table 7.1: Patient and tumor characteristics of cohort 1 , cohort 2 and the combined dataset. 
a resampling to a voxel size that is similar to the voxel size of the training dataset.

As the radiomic signature is based on analysis of the primary tumor, tumor volume might have impact on the outcome. In the stage I-III NSCLC signature cohort of Aerts et al. [16] the median tumor volume was $251 \mathrm{~cm}^{3}$ (2-489), while in our stage IV NSCLC cohort the median tumor volume was only $36 \mathrm{~cm}^{3},(0-769)$. As can be seen in Supplementary Material Figure 7.3, the variation in tumor volumes is much larger in the signature cohort compared to cohort 1 and cohort 2, which have a comparable variation. As seen from the coefficients in the signature model, some parameters have a large correlation to tumor size. In cohort $146 \%$ of the tumors were staged as T4, while in the signature cohort only $28 \%$ was staged as T4. A T4 staged tumor can be a large tumor but it can as well mean that there are multiple nodules in the lung. In case of multiple nodules, the largest lesion was used for the radiomic analysis, which can also explain the difference in tumor volumes between the two cohorts.

Our cohorts have an equal distribution of cT-stage compared to Aerts et al. [16], which confirms that cT-stage is not an indicator of tumor volume. In stage IV NSCLC mainly the cM-stage determines the prognosis of a patient. With radiomics image characteristics of the primary tumor but also of the nodes and metastases can be easily

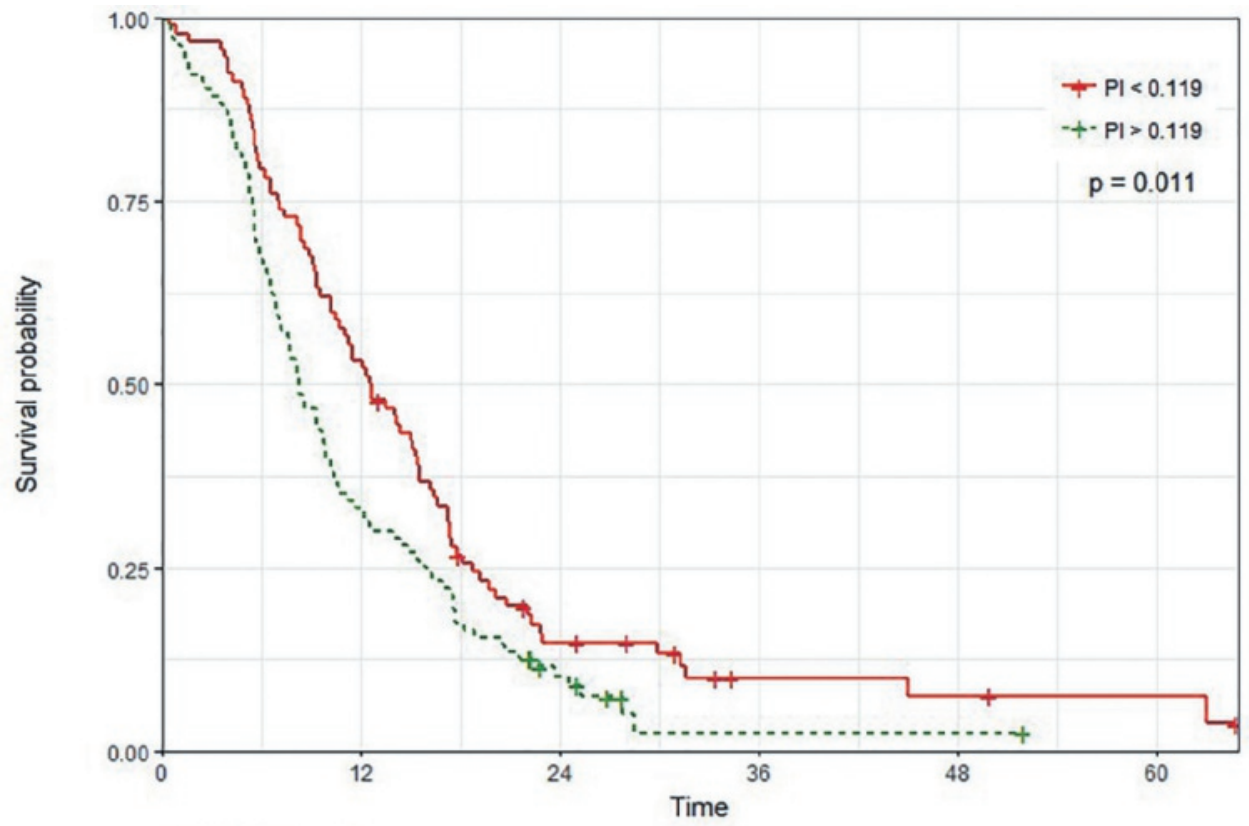

Number at risk

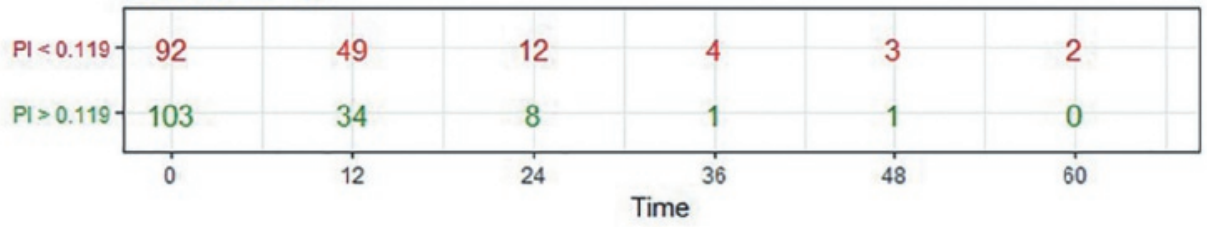

Figure 7.2: Kaplan Meier plot of overall survival showing the prognostic index of the radiomic signature split at the median of the Nature signature. 


\begin{tabular}{l|l|l|l}
\hline Clinical variables & & $\mathrm{HR}(95 \% \mathrm{Cl})$ & $\mathrm{p}$-value \\
\hline Age & & $0.996(0.980-1.012)$ & 0.633 \\
Gender & & $0.761(0.565-1.025)$ & 0.072 \\
WHO-PS & & & 0.038 \\
& WHO 1 & $1.521(1.095-2.114)$ & 0.012 \\
cT-stage & WHO 2 & $1.159(0.717-1.872)$ & 0.547 \\
& & & 0.454 \\
& T2 & $1.288(0.800-2.075)$ & 0.297 \\
& T3 & $1.226(0.727-2.069)$ & 0.444 \\
cN-stage & T4 & $1.545(0.971-2.460)$ & 0.066 \\
& Tx & $1.216(0.599-2.468)$ & 0.588 \\
& & & 0.045 \\
& N1 & $1.363(0.650-2.860)$ & 0.412 \\
cM-stage & N2 & $1.256(0.783-2.014)$ & 0.344 \\
& N3 & $1.837(1.152-2.930)$ & 0.011 \\
PI & Nx & $2.159(0.998-4.672)$ & 0.051 \\
& M1b & $0.929(0.645-1.336)$ & 0.016 \\
& M1c & $1.534(1.074-2.189)$ & 0.019 \\
& & $1.464(1.021-2.101)$ & 0.038 \\
\hline
\end{tabular}

PS performance status, cT clinical Tumor-stage, cN clinical Nodal-stage, $\mathrm{cM}$ clinical Metastases-stage, HR hazard ratio,

$\mathrm{Cl}$ confidence interval, OS overall survival, PI prognostic index.

Table 7.2: Hazard ratios with $95 \%$ confidence intervals of the univariable cox regression of the clinical variables and the PI for OS, with uncorrected p-values.

used for prognostic models. To make better models in the future, large datasets are needed, and therefore the tumor segmentation process should be automated. Using deep learning, the image segmentation can be automated, which makes the process less time consuming. A recent publication by Antropova et al. [26] used a mixed approach, combining deep learning and radiomics, and showed that the combined approach outperformed the individual approaches. Song et al. [27] developed and validated a nomogram including radiomic features for predicting progression free survival in stage IV EGFR-mutant NSCLC. They found a model performance of $0.71(0.64-0.77)$ for the training dataset and a comparable performance for the validation dataset 0.72 (0.65-0.78). A study of Coroller et al. [3] showed that a model consisting of CT-based radiomic features was able to predict distant metastases in stage I-III NSCLC patients, with a model performance of 0.61 in an independent validation cohort.

Our study shows that a radiomic signature applied on a routine baseline CT scan has prognostic value for stage IV NSCLC patients. In the future, radiomics in combination with machine learning or deep learning can potentially lead to better prognostic models in stage IV NSCLC. Next to radiomics of the primary tumor, normal lung radiomics can give additional information to existing clinical prognostic factors. 


\begin{tabular}{l|l|l|l}
\hline Clinical variables & & $\mathrm{HR}(95 \% \mathrm{Cl})$ & $\mathrm{p}$-value \\
\hline Age & & $0.993(0.973-1.013)$ & 0.497 \\
Gender & & $0.738(0.533-1.021)$ & 0.067 \\
WHO-PS & & & 0.169 \\
& WHO 1 & $1.395(0.976-1.995)$ & 0.068 \\
cT-stage & WHO 2 & $1.150(0.652-1.937)$ & 0.673 \\
& & & 0.968 \\
& T2 & $1.193(0.728-1.956)$ & 0.483 \\
& T3 & $1.163(0.658-2.057)$ & 0.603 \\
cN-stage & T4 & $1.170(0.717-1.911)$ & 0.529 \\
& Tx & $1.197(0.573-2.500)$ & 0.632 \\
& & & 0.035 \\
& N1 & $1.204(0.555-2.610)$ & 0.639 \\
cM-stage & N2 & $1.231(0.757-2.000)$ & 0.402 \\
& N3 & $1.915(1.175-3.121)$ & 0.009 \\
& Nx & $2.113(0.924-4.833)$ & 0.076 \\
PI & M1b & $1.003(0.678-1.484)$ & 0.022 \\
& M1c & $1.617(1.110-2.357)$ & 0.012 \\
& & $1.556(1.041-2.325)$ & 0.031 \\
\hline
\end{tabular}

PS performance status, cT clinical Tumor-stage, cN clinical Nodal-stage, cM clinical Metastases-stage, HR hazard ratio,

$\mathrm{Cl}$ confidence interval, OS overall survival, $\mathrm{PI}$ prognostic index.

Table 7.3: Hazard ratios with $95 \%$ confidence intervals of the multivariable cox regression of the clinical variables and the PI for OS, with uncorrected $\mathrm{p}$-values.

\section{Funding}

The NVALT12 is a multicenter randomized open-label parallel group phase II trial conducted by the Dutch Lung Physician Society (NVALT) and was supported by the Dutch Cancer Society under Grant UM 2010-4883.

\section{Disclosure}

RL: none related to current manuscript. Other: personal fees and other from Oncoradiomics (SA), in addition RL has a patent EP320751A1 pending. LH: none related to current manuscript. Other: research funding Roche, ad board Boehringer, BMS, travel reimbursement Amgen, Roche, BMS. PL: none related to current manuscript. Other: grants, non-financial support and other from Oncoradiomics (SA), in addition PL has a patent EP320751A1 with royalties paid. The authors EdJ, WvE, SR, $A C, G S, A J, E T, B R$ and $A D$ report no conflicts of interest.

\section{Acknowledgements}

Author PL acknowledge financial support from ERC advanced grant (ERC-ADG-2015, No. 694812 - Hypoximmuno) and the QulC-ConCePT project, which is partly funded by 
EFPI A companies and the Innovative Medicine Initiative Joint Undertaking (IMI JU) under Grant Agreement No. 115151. Author PL also acknowledge financial support from the Dutch technology Foundation STW (grant No. 10696 DuCAT and No. P14-19 Radiomics STRaTegy), which is the applied science division of NWO, and the Technology Programme of the Ministry of Economic Affairs. Authors also acknowledge financial support from the SME Phase 2 (RAIL - No. 673780), EUROSTARS (DART, DECIDE, COMPACT), the European Program H2020-2015-17 (ImmunoSABR - No. 733008 and PREDICT - ITN - No. 766276), Interreg V-A Euregio Meuse-Rhine ("Euradiomics") and Kankeronderzoekfonds Limburg from the Health Foundation Limburg. 


\section{References}

[1] K. M. Panth et al. "Is there a causal relationship between genetic changes and radiomics-based image features? An in vivo preclinical experiment with doxycycline inducible GADD34 tumor cells". In: Radiother Oncol 116.3 (2015). Radiotherapy and oncology : journal of the European Society for Therapeutic Radiology and Oncology, pp. 462-6. DOI: 10.1016/j.radonc.2015.06.013.

[2] Y. Balagurunathan et al. "Test-retest reproducibility analysis of lung CT image features". In: J Digit Imaging 27.6 (2014). Journal of digital imaging, pp. 805-23. DOI: $10.1007 / \mathrm{s} 10278-014-9716-x$.

[3] T. P. Coroller et al. "CT-based radiomic signature predicts distant metastasis in lung adenocarcinoma". In: Radiother Oncol 114.3 (2015). Radiotherapy and oncology : journal of the European Society for Therapeutic Radiology and Oncology, pp. 345-50. DOI: 10.1016/j.radonc.2015.02.015.

[4] D. V. Fried et al. "Prognostic value and reproducibility of pretreatment CT texture features in stage III non-small cell lung cancer". In: Int J Radiat Oncol Biol Phys 90.4 (2014). International journal of radiation oncology, biology, physics, pp. 834-42. DOI: 10.1016/j.ijrobp.2014.07.020.

[5] B. Ganeshan et al. "Tumour heterogeneity in non-small cell lung carcinoma assessed by CT texture analysis: a potential marker of survival". In: Eur Radiol 22.4 (2012). European radiology, pp. 796-802. DOI: 10.1007/s00330-011-2319-8.

[6] S. X. Rao et al. "Whole-liver CT texture analysis in colorectal cancer: Does the presence of liver metastases affect the texture of the remaining liver?" In: United European Gastroenterol J 2.6 (2014). United European gastroenterology journal, pp. 530-8. DOI: $10.1177 / 2050640614552463$.

[7] H. Zhang et al. "Locally advanced squamous cell carcinoma of the head and neck: CT texture and histogram analysis allow independent prediction of overall survival in patients treated with induction chemotherapy". In: Radiology 269.3 (2013). Radiology, pp. 801-9. DOI: 10.1148/radiol.13130110.

[8] V. Kumar et al. "Radiomics: the process and the challenges". In: Magn Reson Imaging 30.9 (2012). Magnetic resonance imaging, pp. 1234-48. DOI: 10.1016/j.mri.2012.06.010.

[9] P. Lambin et al. "Radiomics: extracting more information from medical images using advanced feature analysis". In: Eur J Cancer 48.4 (2012). European journal of cancer (Oxford, England : 1990), pp. 441-6. DOI: 10.1016/j.ejca.2011.11.036.

[10] P. Lambin et al. "Rapid Learning health care in oncology an approach towards decision support systems enabling customised radiotherapy". In: Radiother Oncol 109.1 (2013). Radiotherapy and oncology : journal of the European Society for Therapeutic Radiology and Oncology, pp. 159-64. DOI: 10.1016/j.radonc.2013.07. 007.

[11] Y. Balagurunathan et al. "Reproducibility and Prognosis of Quantitative Features Extracted from CT Images". In: Transl Oncol 7.1 (2014). Translational oncology, pp. $72-87$. 
[12] P. E. Galavis et al. "Variability of textural features in FDG PET images due to different acquisition modes and reconstruction parameters". In: Acta Oncol 49.7 (2010). Acta oncologica (Stockholm, Sweden), pp. 1012-6. DOI: 10.3109/0284186x. 2010.498437.

[13] P. Lambin et al. "Predicting outcomes in radiation oncology-multifactorial decision support systems". In: Nat Rev Clin Oncol 10.1 (2013). Nature reviews. Clinical oncology, pp. 27-40. DOI: 10.1038/nrclinonc.2012.196.

[14] C. Parmar et al. "Robust Radiomics feature quantification using semiautomatic volumetric segmentation." eng. In: PloS one 9 (7 2014), e102107.

[15] E. Rios Velazquez et al. "A semiautomatic CT-based ensemble segmentation of lung tumors: comparison with oncologists' delineations and with the surgical specimen". In: Radiother Oncol 105.2 (2012). Radiotherapy and oncology : journal of the European Society for Therapeutic Radiology and Oncology, pp. 167-73. DOI: $10.1016 / \mathrm{j}$. radonc.2012.09.023.

[16] H. J. Aerts et al. "Decoding tumour phenotype by noninvasive imaging using a quantitative radiomics approach". In: Nat Commun 5 (2014). Nature communications, p. 4006. DOI: $10.1038 /$ ncomms5006.

[17] R. T. Leijenaar et al. "External validation of a prognostic CT-based radiomic signature in oropharyngeal squamous cell carcinoma". In: Acta Oncol 54.9 (2015). Acta oncologica (Stockholm, Sweden), pp. 1423-9. DOI: 10.3109/0284186x.2015.1061214.

[18] W. E. Eberhardt et al. "The IASLC Lung Cancer Staging Project: Proposals for the Revision of the M Descriptors in the Forthcoming Eighth Edition of the TNM Classification of Lung Cancer". In: J Thorac Oncol 10.11 (2015). Journal of thoracic oncology : official publication of the International Association for the Study of Lung Cancer, pp. 1515-22. DOI: 10.1097/jto.0000000000000673.

[19] L. E. Hendriks et al. "Single organ metastatic disease and local disease status, prognostic factors for overall survival in stage IV non-small cell lung cancer: Results from a population-based study". In: Eur J Cancer 51.17 (2015). European journal of cancer (Oxford, England : 1990), pp. 2534-44. DOI: 10.1016/j.ejca.2015.08.008.

[20] S. Rizzo et al. "CT Radiogenomic Characterization of EGFR, K-RAS, and ALK Mutations in Non-Small Cell Lung Cancer". In: Eur Radiol 26.1 (2016). European radiology, pp. 32-42. DOI: 10.1007/s00330-015-3814-0.

[21] A. M. Dingemans et al. "A randomized phase II study comparing paclitaxel-carboplatin-bevacizumab with or without nitroglycerin patches in patients with stage IV nonsquamous nonsmall-cell lung cancer: NVALT12 (NCT01171170)". In: Ann Oncol 26.11 (2015). Annals of oncology : official journal of the European Society for Medical Oncology, pp. 2286-93. DOI: 10.1093/annonc/mdv370.

[22] J. O. Deasy, A. I. Blanco, and V. H. Clark. "CERR: a computational environment for radiotherapy research". In: Med Phys 30.5 (2003). Medical physics, pp. 979-85. DOI: $10.1118 / 1.1568978$.

[23] R. T. H. M. Larue et al. "Influence of gray level discretization on radiomic feature stability for different CT scanners, tube currents and slice thicknesses: a comprehensive phantom study". In: Acta Oncol In press (2017), p. 11. DOI: 10.1080/0284186X.2017.1351624. 
[24] M. Shafiq-UI-Hassan et al. "Intrinsic dependencies of CT radiomic features on voxel size and number of gray levels". In: Med Phys 44.3 (2017). Medical physics, pp. 1050-1062. DOI: 10.1002/mp.12123.

[25] D. Mackin et al. "Harmonizing the pixel size in retrospective computed tomography radiomics studies". In: PLoS One 12.9 (2017). PloS one, e0178524. DOI: 10.1371/ journal.pone.0178524.

[26] N. Antropova et al. "A deep feature fusion methodology for breast cancer diagnosis demonstrated on three imaging modality datasets." eng. In: Medical physics 44 (10 2017), pp. 5162-5171.

[27] J. Song et al. "Development and validation of a radiomics nomogram for progressionfree survival prediction in stage IV EGFR-mutant non-small cell lung cancer". In: SPIE Medical Imaging. Vol. 10134. SPIE, 2017, p. 6. 


\section{Supplementary Material}

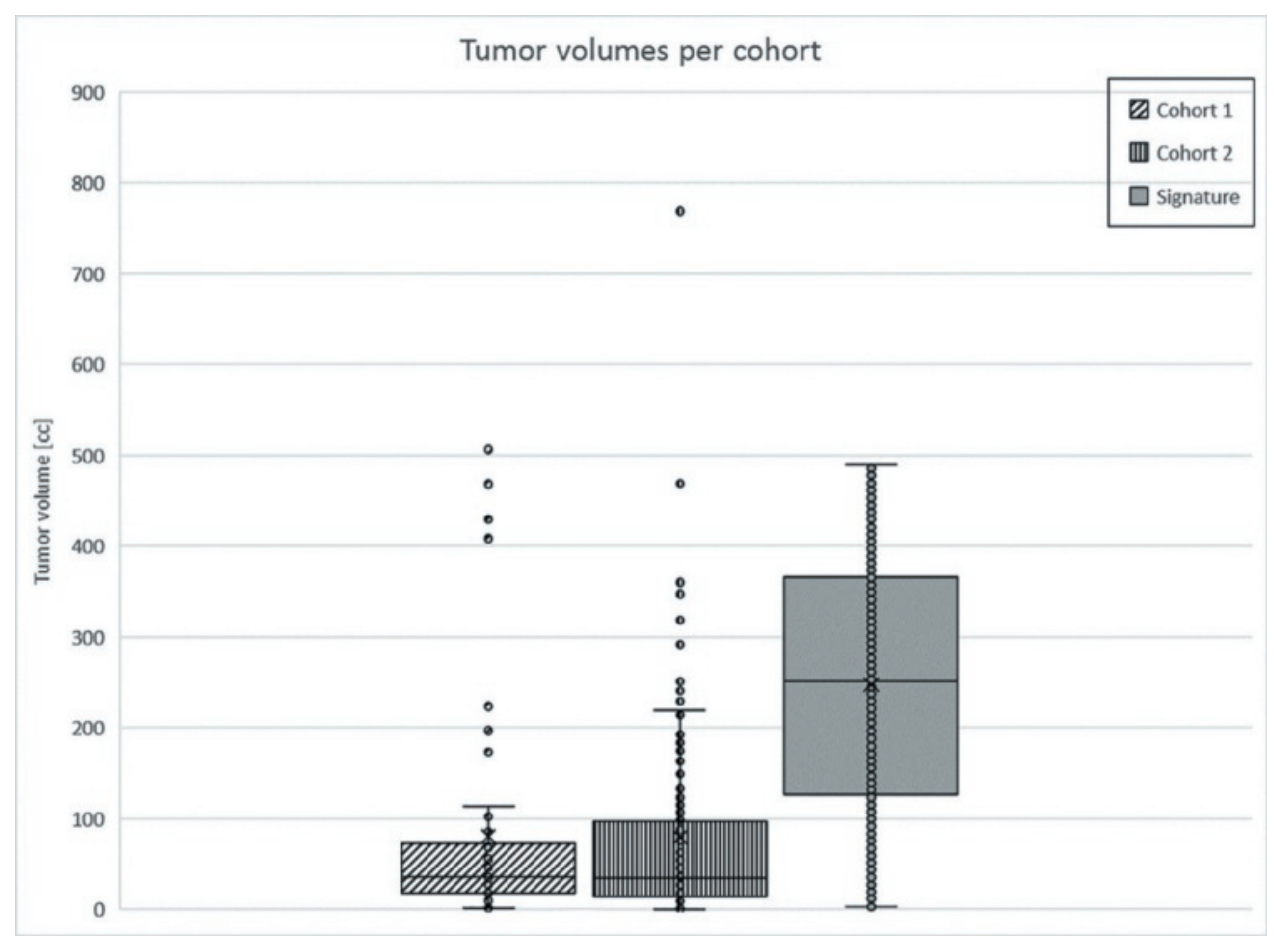

Figure 7.3: Boxplot of the tumor volumes of cohort 1, cohort 2 and the signature cohort [2]. 


\begin{tabular}{llll}
\hline Clinical variables & & HR $\mathbf{( 9 5} \mathbf{~ o ~ C l})$ & p-value \\
\hline Age & & $0.991(0.972-1.011)$ & 0.380 \\
Gender & & $0.713(0.514-0.988)$ & 0.042 \\
WHO-PS & & & 0.199 \\
& WHO1 & $1.380(0.965-1.973)$ & 0.078 \\
cT-stage & WHO2 & $1.157(0.672-1.993)$ & 0.598 \\
& & & 0.896 \\
& T2 & $1.229(0.751-2.008)$ & 0.412 \\
& T3 & $1.326(0.761-2.309)$ & 0.319 \\
cN-stage & T4 & $1.251(0.769-2.035)$ & 0.368 \\
& Tx & $1.181(0.566-2.466)$ & 0.657 \\
& & & 0.064 \\
& N1 & $1.129(0.523-2.435)$ & 0.758 \\
cM-stage & N2 & $1.228(0.757-1.993)$ & 0.406 \\
& N3 & $1.802(1.114-2.915)$ & 0.016 \\
& Nx & $2.068(0.904-4.730)$ & 0.085 \\
& M1b & $1.014(0.686-1.499)$ & 0.015 \\
& M1c & $1.668(1.145-2.430)$ & 0.008 \\
\hline
\end{tabular}

Table 7.4: Hazard ratios with $95 \%$ confidence intervals of the multivariable cox regression of the clinical variables for OS, with uncorrected p-values. 



\section{Chapter 8}

Can radiomic features describe lung semantic features in non-small cell lung cancer patients?

Authors

Evelyn E.C. de Jong, Timo M. Deist, Wouter van Elmpt, Arthur Jochems, Stefania Rizzo, Francesco Petrella, Massimo Bellomi, Ralph T.H. Leijenaar, Turkey A.G. Refaee, Lizza E.L. Hendriks, Bart Reymen, Anne-Marie C. Dingemans, Philippe Lambin

In preparation 


\section{Abstract}

Qualitative image characteristics, such as cavitation or air bronchogram, have been shown to be prognostic for treatment outcome in non-small cell lung cancer (NSCLC). However, scoring of these features manually might suffer from inter-observer variability. We hypothesized that automatically calculated radiomic features could objectively describe these semantic features. Therefore, in this study the correlation between 15 qualitative semantic features and 160 quantitative radiomic features of the primary tumor and 201 of the ipsilateral lung, was investigated in 218 NSCLC patients. In addition, we developed models to describe these semantic features, based on combinations of radiomic features, which were validated in an independent cohort of 98 NSCLC patients. Tumor radiomic features, could predict the semantic features air bronchogram, necrosis and pleural effusion (AUC 0.71, 0.68, and 0.65 , respectively). Lung radiomic features could predict pleural thickening, satellite nodules and pleural effusion (AUC $0.62,0.64$, and 0.87 , respectively). For combinations of tumor and lung radiomic features the performance was for air bronchogram, pleural thickening, necrosis, satellite nodules and pleural effusion respectively AUC $0.68,0.66,0.72,0.65$ and 0.83 . Although there are associations between semantic and radiomic features, larger training datasets with new approaches, like deep learning, are needed to further improve performance. 


\section{Introduction}

Medical imaging, like computed tomography (CT), is routinely used for diagnosis and response assessment procedures in patients with non-small cell lung cancer (NSCLC). Medical imaging is, in contrast to pathological investigations, non-invasive and can be applied at multiple time-points before and during treatment [1].

The survival rate of NSCLC patients is still unsatisfactory and dependent on stage. However, even within a certain stage, survival varies. Prognostic models, consisting of clinical parameters and genetic information, can make treatment more personalized and potentially increase the survival rate [2].

More and more targetable molecular alterations are being discovered. Examples are epidermal growth factor receptor (EGFR), anaplastic lymphoma kinase (ALK), ROS1, BRAF, for which tyrosine kinase inhibitors (TKIs) have been approved [3]. However, tissue acquisition through invasive biopsies cannot represent the complete tumor genotype.

A study of Rizzo et al. [4] showed, in a dataset of 285 patients with lung adenocarcinoma that were tested for EGFR, ALK and KRAS mutations, that single qualitative image features as cavitation, emphysema, ground glass opacity, air bronchogram, necrosis, pleural retraction, pleural effusion, pleural thickening, pleural contact, fibrosis and satellite nodules are shown to be predictive for mutation status. Other studies showed that those semantic features are also prognostic for survival $[5,6]$. However, these semantic features are scored by a visual assessment of radiologists and might be inter-observer dependent and time consuming when applied to large datasets.

Radiomics, which is a high-throughput approach to automatically extract a large number of quantitative image features, is as well shown to be prognostic [7]. A radiomic signature, existing of four radiomic features, developed in 422 stage I-III NSCLC patients, which was prognostic for overall survival, could be externally validated in a cohort of 225 stage I-III NSCLC patients. Next to that, this signature was also prognostic for overall survival in head and neck cancer [7]. Using radiomics the image phenotype can be quantified, which is different for tumors with somatic mutations $[8,9]$. A study of Yip et al. [10] recently found, in a cohort of 183 early stage and 75 advanced stage NSCLC patients, associations between qualitative semantic features and radiomic features. They focused on semantic and radiomic features scored for the tumor while it is known that patients with lung abnormalities usually have a worse prognosis [10]. Tumors that are attached to vessels, an unclear border definition but as well pleural retraction are associated with a worse overall survival [11].

In the current study, we investigated associations between semantic features and radiomic features of the tumor in a cohort of NSCLC patients and investigated the correlation between semantic features and radiomic features of the ipsilateral lung. By using combinations of radiomic features we hypothesize that the models improved by describing the semantic features. The developed models are validated using an independent cohort of stage IV NSCLC patients.

\section{Methods}

\section{Patient characteristics}

For training, a cohort of 285 advanced stage NSCLC patients (cohort A), which were retrospectively selected from a database with lung adenocarcinoma patients tested for 
EGFR mutation/KRAS mutation/ anaplastic lymphoma kinase (ALK) translocation, was used (see Rizzo et al. [4] for patient inclusion criteria and treatment specifications). Written informed consent to the use of clinical and imaging data for scientific purposes was obtained from all patients beforehand.

For validation, a cohort of 271 advanced stage NSCLC patients (cohort B), was retrospectively selected from a database with lung adenocarcinoma patients tested for EGFR mutation/KRAS mutation/ALK translocation. The data was retrospectively collected with approval of the Institutional Review Board of the Department of Radiation Oncology of Maastricht University Medical Center (MAASTRO Clinic) in accordance with the informed consent form and/or the applicable Dutch and international rules and legislation for handling of medical and personal data. Data was processed by the investigator in an anonymized manner. All procedures performed in this study were in accordance with the ethical standards of the institutional and national research committee and with the 1964 Helsinki Declaration and its later amendments.

Patients that had an unknown status for one or more semantic features were excluded, as were patients with a CT-scan without a definable primary tumor, a too small field of view, an original tumor volume that was separated by the resampling procedure or a non-standard patient position.

\section{Imaging}

For the training cohort all CT scans were performed in the same hospital using a 16-slice Lightspeed GE CT scanner or a 64-slice MSTC Optima 660 GE CT scanner. Diagnostic CT scans with or without contrast were performed. A standard thorax protocol was used with a mean slice thickness of $3 \mathrm{~mm}$.

For the validation cohort the CT scans were performed in nine different centers, using four different CT scanner brands (Philips, Siemens, Toshiba, GE) and 14 different CT scanner types. The slice thickness varied from 2 to $5 \mathrm{~mm}$ with a mean slice thickness of $4.5 \mathrm{~mm}$.

For all scans the primary tumor was manually delineated by an experienced radiation oncologist (BR) using Mirada (Mirada Medical, Oxford, UK) and used as the region of interest. The lungs were delineated using atlas based contouring (Workflowbox Mirada Medical, Oxford, UK) and were manually adjusted if needed. On the scans where the lungs could not be delineated using atlas based contouring, threshold based contouring was used with a threshold from -1000 to $-200 \mathrm{HU}$ and manually adjusted afterwards if needed.

\section{Semantic features}

Qualitative features were scored as described in Rizzo et al. [4]. For each CT, the following qualitative features were scored:

i presence (1) or absence (0) of a ground-glass opacity;

ii lesion density, indicated as sub-solid (0) or solid (1);

iii presence (1) or absence (0) of cavitation;

iv presence (1) or absence (0) of air bronchogram;

$v \quad$ thickening of the adjacent pleura (yes $=1$, no $=0$ );

vi presence (1) or absence (0) of intra-tumoral necrosis;

vii presence (1) or absence (0) of satellite nodules in primary tumor lobe;

viii presence (1) or absence (0) of nodules in non-tumor lobes; 
ix presence (1) or absence (0) of pleural retraction;

$\times \quad$ location of the lesion, as central (1) or peripheral (0);

$\mathrm{xi}$ presence (1) or absence (0) of intra-nodular calcifications;

xii presence (1) or absence (0) of fibrosis;

xiii presence (1) or absence (0) of pleural contact;

xiv presence $(1)$ or absence $(0)$ of pleural effusion.

All semantic features were scored on a binary scale (see Table 8.1).

\begin{tabular}{|c|c|c|c|c|c|}
\hline \multicolumn{2}{|l|}{ Semantic feature } & \multicolumn{2}{|c|}{ Cohort A $n=218(\%)$} & \multicolumn{2}{|c|}{ Cohort B $n=98(\%)$} \\
\hline \multirow[t]{2}{*}{ GGO } & Absence & 175 & $(80 \%)$ & 87 & $(89 \%)$ \\
\hline & Presence & 43 & $(20 \%)$ & 11 & $(11 \%)$ \\
\hline \multirow[t]{2}{*}{ Density } & Subsolid & 25 & $(11 \%)$ & 7 & $(8 \%)$ \\
\hline & Solid & 193 & $(89 \%)$ & 90 & $(92 \%)$ \\
\hline \multirow[t]{2}{*}{ Cavitation } & Absence & 186 & $(85 \%)$ & 90 & $(92 \%)$ \\
\hline & Presence & 32 & $(15 \%)$ & 8 & $(8 \%)$ \\
\hline \multirow[t]{2}{*}{ Air bronchogram } & Absence & 126 & $(58 \%)$ & 63 & $(64 \%)$ \\
\hline & Presence & 92 & $(42 \%)$ & 35 & $(36 \%)$ \\
\hline \multirow[t]{2}{*}{ Pleural thickening } & Absence & 114 & $(52 \%)$ & 42 & $(43 \%)$ \\
\hline & Presence & 104 & $(48 \%)$ & 56 & $(57 \%)$ \\
\hline \multirow[t]{2}{*}{ Necrosis } & Absence & 172 & $(79 \%)$ & 77 & $(79 \%)$ \\
\hline & Presence & 46 & $(21 \%)$ & 21 & $(21 \%)$ \\
\hline \multirow[t]{2}{*}{ Satellite nodules } & Absence & 148 & $(68 \%)$ & 61 & $(62 \%)$ \\
\hline & Presence & 70 & $(32 \%)$ & 37 & $(38 \%)$ \\
\hline \multirow[t]{2}{*}{ Suspect nodules } & Absence & 135 & $(62 \%)$ & 52 & $(53 \%)$ \\
\hline & Presence & 83 & $(38 \%)$ & 46 & $(47 \%)$ \\
\hline \multirow[t]{2}{*}{ Pleural retraction } & Absence & 105 & $(48 \%)$ & 24 & $(24 \%)$ \\
\hline & Presence & 113 & $(52 \%)$ & 74 & $(76 \%)$ \\
\hline \multirow[t]{2}{*}{ Tumor location } & Peripheral & 137 & $(63 \%)$ & 52 & $(53 \%)$ \\
\hline & Central & 81 & $(37 \%)$ & 46 & $(47 \%)$ \\
\hline \multirow[t]{2}{*}{ Calcifications } & Absence & 191 & $(88 \%)$ & 95 & $(97 \%)$ \\
\hline & Presence & 27 & $(12 \%)$ & 3 & $(3 \%)$ \\
\hline \multirow[t]{2}{*}{ Emphysema } & No & 184 & $(84 \%)$ & 69 & $(70 \%)$ \\
\hline & Yes & 34 & $(16 \%)$ & 29 & $(30 \%)$ \\
\hline \multirow[t]{2}{*}{ Fibrosis } & Absence & 190 & $(87 \%)$ & 85 & $(87 \%)$ \\
\hline & Presence & 28 & $(13 \%)$ & 13 & $(13 \%)$ \\
\hline \multirow[t]{2}{*}{ Pleural contact } & Absence & 78 & $(36 \%)$ & 26 & $(27 \%)$ \\
\hline & Presence & 140 & $(64 \%)$ & 72 & $(73 \%)$ \\
\hline \multirow[t]{2}{*}{ Pleural effusion } & Absence & 186 & $(85 \%)$ & 71 & $(72 \%)$ \\
\hline & Presence & 32 & $(15 \%)$ & 27 & $(28 \%)$ \\
\hline
\end{tabular}

GGO ground glass opacity.

Table 8.1: Number of events per semantic feature for cohort A en cohort B.

\section{Radiomic features}

Image features were calculated on the baseline CT scan using an adapted version of Computational Environment for Radiotherapy Research (CERR) extended with in-house developed radiomic image analysis software (Matlab 2014a, The Mathworks, Natick, 
MA) to extract the image features $[12,13]$. A bin width of 25 Hounsfield Units was used. To minimize the possible effect of the variation in image parameters, all scans were resampled to a voxel size of $1 \times 1 \times 3 \mathrm{~mm}^{3}$ using a cubic interpolation as recommended in Larue et al. [14]. In total 1322 features, 24 shape features, 19 statistics features and 127 textural features (26 GLCM, 16 GLDZM, 16 GLRLM, 16 GLSZM, 26 IH, 17 NGLDM, 5 NGTDM, 2 Local Intensity and 3 Fractal features) were extracted for the primary tumor and for the tumor lung. Next to that, 1152 features were calculated after applying a wavelet filter. A mathematical description of all features can be found in the Supplementary Material Figure 8.5.

\section{Radiomic feature selection}

Spearman's correlation coefficient $(\rho)$ was used to assess the correlation between all radiomic features. Features with $|\rho| \geq 0.85$ were considered to be strongly correlated and likely to provide redundant information about the tumor phenotype. In these strongly correlated pairs, the feature with the highest average $|\rho|$ was excluded. Next to that, features with unique values and with missing values were excluded.

\section{Statistics}

\section{Univariable}

The univariable analysis is performed in RStudio (version 3.4.1). The association between radiomic features and semantic features was assessed using the area under the receiver operating curve (AUC). Noether's test was used to determine significance of the AUC from a random relationship $(A \cup C=0.5)$. All $p$-values were corrected for multiple testing by adjusting the false discovery rate according to the Benjamini and Hochberg procedure, where a q-value $<0.05$ suggested statistical significance.

\section{Multivariable}

The multivariable analysis is performed in Matlab R2018a (The Mathworks, Natick, MA). The analysis is conducted separately for each of the 15 semantic features, and repeated for three sets of predictors: for radiomic features of the primary tumor, radiomic features of the ipsilateral lung, and the combination of radiomics features of the primary tumor and the ipsilateral lung. This results in a total of 45 separate analyses. Each time, a random forest model is trained on the training dataset and validated on the validation dataset. The default hyper parameter set was used and the number of trees was set to 200. Missing radiomic feature values in the validation set were imputed by the training set mean.

Performance was expressed in the AUC with a $95 \%$ confidence interval computed from 100 bootstraps. ROC curves were plotted for pleural effusion, for radiomic features of the tumor, lung and a combination of radiomic features. For every analysis, the top ten ranked radiomic features were listed. The radiomic features selected in the tumor and lung analysis were compared to the radiomic features selected in the combined analysis. 


\section{Results}

\section{Patient selection}

For 249 out of 285 patients from the training cohort a baseline CT scan could be retrieved. Thirty-one scans were excluded from analysis, reasons were: a non-measurable primary tumor $(n=4)$, an incorrect patient position $(n=1)$, a too small field of view $(n=5)$, a separated volume due to the resampling $(n=13)$ and an unknown semantic feature status for at least one semantic feature $(n=8)$. In total 218 scans could be analyzed.

For the validation cohort a baseline CT scan was received from 131 patients. Thirtythree scans were excluded, reasons were: a too small field of view $(n=1)$, a non-measurable primary tumor $(n=2)$, a separated volume due to the resampling $(n=13)$ and an unknown semantic feature status for at least one semantic feature $(n=17)$. In total 98 scans could be used for the analysis (see Figure 8.1 for the CONSORT-diagram).

TRAINING

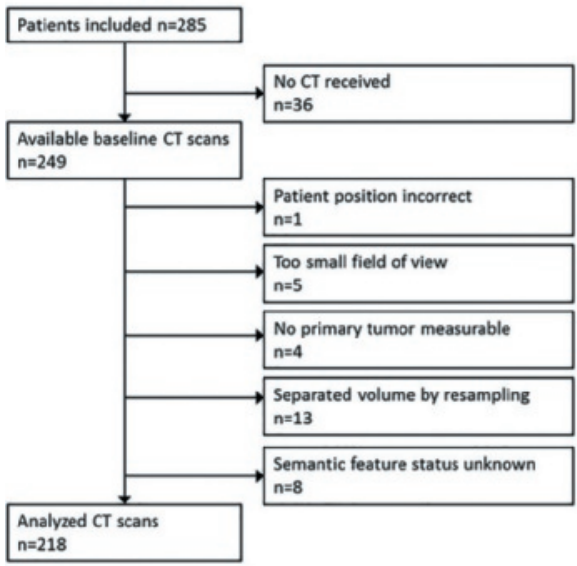

\section{VALIDATION}

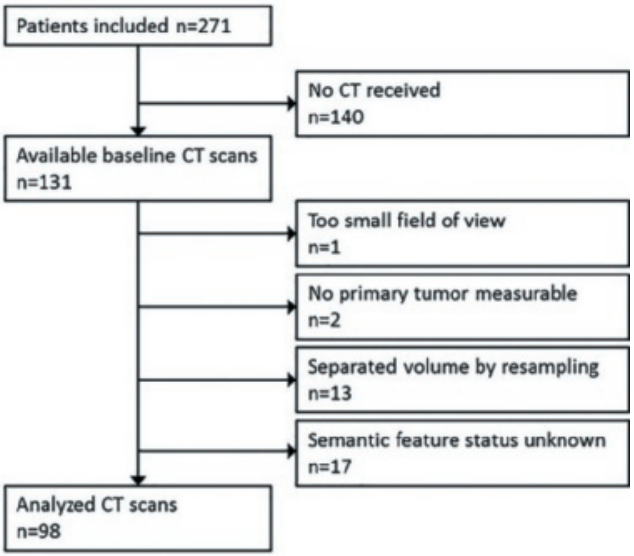

Figure 8.1: CONSORT diagram of cohort A (left) and the cohort B (right).

\section{Feature selection}

Excluding the radiomic features that contained redundant information or unique values, left for the primary tumor 18 unfiltered radiomic features ( 7 shape, 2 statistics, 1 GLCM, 1 GLDZM, 2 GLSZM, 1 fractal, 1 IH, 2 Local Intensity, 1 NGLDM), and 142 filtered radiomic features for the analysis.

Excluding the radiomic features that contained redundant information or unique values, left for the ipsilateral lung 36 unfiltered radiomic features (11 shape, 2 statistics, 3 GLCM, 4 GLDZM, 2 GLSZM, 1 GLRLM, 1 fractal, 5 IH, 2 local intensity, 4 NGLDM, 1 NGTDM), and 165 filtered radiomic features for the analysis.

\section{Univariable analysis}

There was a clear association between unfiltered radiomic features of the primary tumor and the semantic features: density, cavitation, air bronchogram, pleural thickening, necrosis, 
satellite nodules, and pleural effusion (see Figure 8.2). For the ipsilateral lung unfiltered radiomic features were associated with the semantic features: density, pleural contact and pleural effusion (see Figure 8.3). Due to the large number of filtered features, the associations between the filtered radiomic features of the tumor as well as the ipsilateral lung with the semantic features can be found in Supplementary Material Figure 8.6 and Figure 8.7.

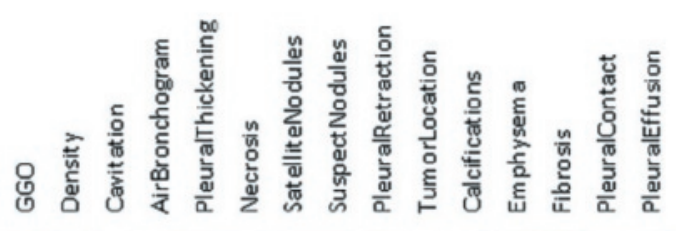

Feature
Fractal_sd
GLOM_maxCorr
GLDZM_LIDE
GLSZM_IV
GLSZM_ZE
IH_mingradi
LocInt_peakGlobal
LocInt_peakLocal
NGDM_LGDE
Shape_areaDensityBB
Shape_areaDensityBE
Shape_centroidDistance
Shape_elongation
Shape_flatness
Shape_sphericity
Shape_volumeDensityBE
Stats_median
Stats_p90
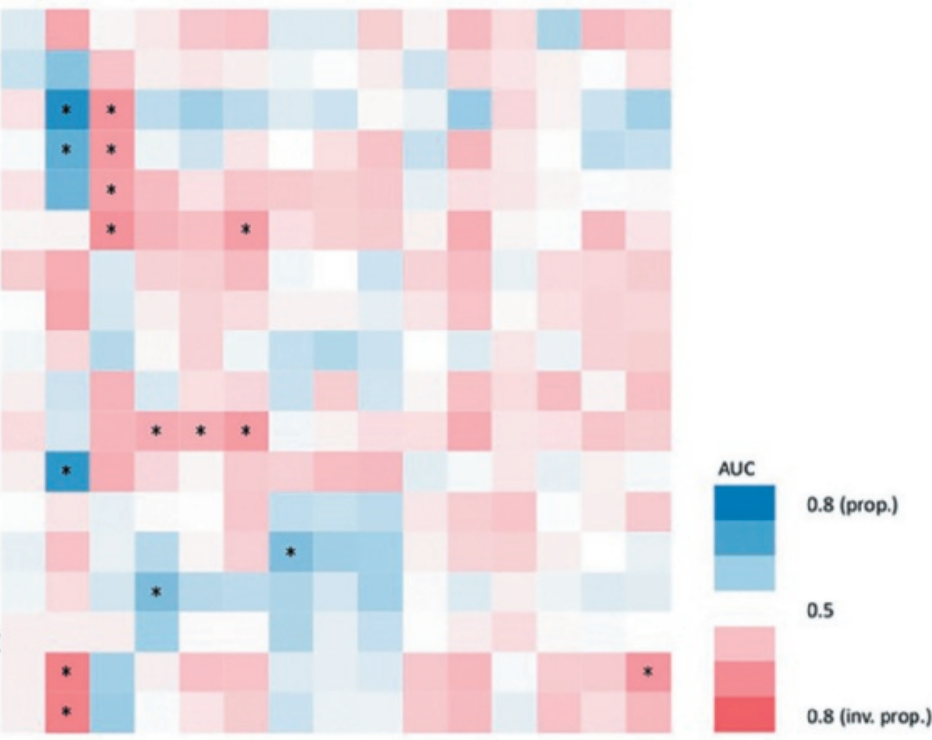

Figure 8.2: Associations between the binary semantic and unfiltered radiomic features assessed with the AUC for the primary tumor. * indicates q-values $<0.05$. AUC: area under the curve, GGO: ground glass opacity.

\section{Multivariable analysis}

The model performance per semantic feature for a combination of radiomic features of the primary tumor, the ipsilateral lung and a combination of tumor and lung features is shown in Table 8.2. The semantic features air bronchogram (AUC 0.71, $95 \% \mathrm{Cl} 0.57$ $0.81)$, necrosis $(0.68,0.52-0.77)$ and pleural effusion $(0.65,0.52-0.79)$ were described by a combination of radiomic features of the tumor (see Supplementary Material Table 8.3 for the top ten selected features). The semantic features pleural thickening (AUC 0.62, $95 \%$ $\mathrm{Cl} 0.51-0.69)$, satellite nodules $(0.64,0.53-0.74)$ and pleural effusion $(0.87,0.72-0.92)$ were described by a combination of radiomic features of the ipsilateral lung (see Supplementary Material Table 8.3 for the top ten selected features). Using a combination of radiomic 
features of the tumor and the ipsilateral lung air the performance is slightly different, air bronchogram AUC 0.68 (95\% Cl 0.56-0.78), pleural thickening 0.66 (0.52-0.73), necrosis $0.72(0.61-0.83)$, satellite nodules $0.65(0.53-0.77)$ and pleural effusion $0.83(0.69-0.88)$.

Feature

Fractal_sd

GLCM_clusProm

GLCM_diffVar

GLCM_maxprob

GLDZM_DZN

GLDZM_HILDE

GLDZM_HISDE

GLDZM_USDE

GLRLM_LRHGE

GLSZM_INN

GLSZM_SAE

IH_cov

IH_maxgradi

IH_mediand

IH_mode

IH_qcod

Locint_peakGlobal

Locint_peakLocal

NGLDM_DN

NGLDM_DV

NGLDM_HGLDE

NGLDM_LGSDE

NGTDM_contrast

Shape_areaDensityBB

Shape_areaDensityBE

Shape_centroidDistance

Shape_elongation

Shape_flatness

Shape_leastaxislength

Shape_majoraxislength

Shape_maxDiameter 2D3

Shape_sphericity

Shape_volumeDensityBB

Shape_volumeDensityBE

Stats_min

Stats_p10
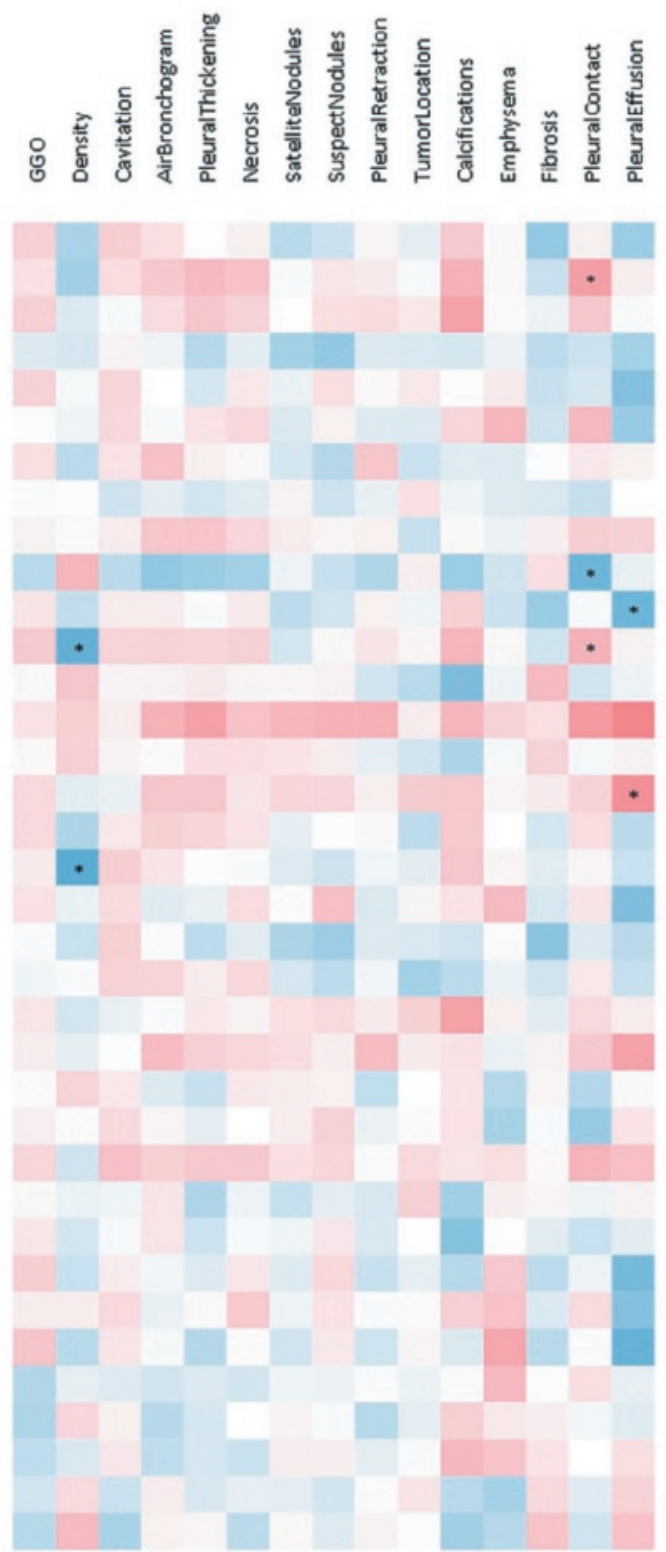

Figure 8.3: Associations between the binary semantic and unfiltered radiomic features assessed with the AUC for the ipsilateral lung. * indicates q-values $<0.05$. AUC: area under the curve, GGO: ground glass opacity. 
In Figure 8.4, the ROC curve for pleural effusion for tumor features, lung features and a combination of features is shown.

\begin{tabular}{l|l|l|l|l|l|c|} 
& \multicolumn{2}{|c|}{ Tumor } & \multicolumn{2}{c|}{ Lung } & \multicolumn{2}{c|}{ Combined } \\
Semantic feature & AUC & $\mathbf{9 5} \%$ Cl AUC & AUC & $\mathbf{9 5} \%$ CI AUC & AUC & 95 \% CI AUC \\
\hline GGO & 0.56 & $0.43-0.71$ & 0.52 & $0.28-0.71$ & 0.56 & $0.38-0.71$ \\
Density & 0.66 & $0.30-0.88$ & 0.53 & $0.16-0.69$ & 0.67 & $0.34-0.91$ \\
Cavitation & 0.72 & $0.42-0.86$ & 0.49 & $0.22-0.72$ & 0.65 & $0.49-0.80$ \\
Air bronchogram & $0.71^{*}$ & $0.57-0.81$ & 0.56 & $0.46-0.70$ & $0.68^{*}$ & $0.56-0.78$ \\
Pleural thickening & 0.56 & $0.46-0.69$ & $0.62^{*}$ & $0.51-0.69$ & $0.66^{*}$ & $0.52-0.73$ \\
Necrosis & $0.68^{*}$ & $0.52-0.77$ & 0.60 & $0.42-0.72$ & $0.72^{*}$ & $0.61-0.84$ \\
Satellite nodules & 0.56 & $0.46-0.67$ & $0.64^{*}$ & $0.53-0.74$ & $0.65^{*}$ & $0.53-0.77$ \\
Suspect nodules & 0.48 & $0.36-0.57$ & 0.55 & $0.42-0.66$ & 0.54 & $0.40-0.65$ \\
Pleural retraction & 0.45 & $0.36-0.58$ & 0.58 & $0.45-0.70$ & 0.55 & $0.40-0.69$ \\
Tumor location & 0.52 & $0.38-0.62$ & 0.47 & $0.35-0.59$ & 0.56 & $0.44-0.67$ \\
Calcifications & 0.74 & $0.41-0.97$ & 0.50 & $0.06-0.75$ & 0.63 & $0.26-0.99$ \\
Emphysema & 0.44 & $0.28-0.53$ & 0.59 & $0.46-0.71$ & 0.52 & $0.40-0.65$ \\
Fibrosis & 0.48 & $0.36-0.62$ & 0.40 & $0.25-0.57$ & 0.39 & $0.22-0.52$ \\
Pleural contact & 0.61 & $0.48-0.75$ & 0.57 & $0.45-0.74$ & 0.56 & $0.42-0.68$ \\
Pleural effusion & $0.65 *$ & $0.52-0.79$ & $0.87^{*}$ & $0.72-0.92$ & $0.83^{*}$ & $0.69-0.88$ \\
\hline
\end{tabular}

GGO ground glass opacity, AUC area under the curve, $\mathrm{Cl}$ confidence interval.

Table 8.2: AUC of the random forest for the validation cohort per semantic feature. The stars represent the significant AUC values (lower bound $95 \% \mathrm{Cl}$ above 0.5) (Random Forest, fitted on training set, validated on validation set).

\section{Discussion}

Previous studies have shown that qualitative image characteristics such as cavitation, ground glass opacity or air bronchogram, are prognostic for treatment outcome in NSCLC $[4,10,11,15,16]$. However, these semantic features suffer from inter-observer variability, because they are scored by a human and not automatically calculated 14 . In this study, we investigated the correlation between visually classified semantic features and quantitative radiomic features. This correlation is investigated for radiomic features of the tumor and for radiomic features of the ipsilateral lung, as we hypothesized that lung features can also describe tumor characteristics and vice-versa.

In the univariable analysis we found like Yip et al. [10] that there is an association between air bronchogram and the tumor radiomic feature shape_sphericity. In the study of Yip et al. [10] as well as in our study the radiomic features that are highly correlated to each other were removed. Comparing the features that are left after removing the highly correlated features shows similar features. Another method to select robust radiomic features is by using a test-retest dataset or a multiple delineation dataset as shown by van Timmeren et al. [17]. This can be performed using an open source test-retest dataset, which makes the results of different studies more comparable. Although in our univariable analysis, different radiomic features were significant for the semantic features compared to Yip et al. [10]. This can be due to a different number of events per semantic feature in both datasets. Collins et al. [18] showed that to reliably validate a prognostic model a minimum of 100 events and ideally 200 or more events are required, which was unfortunately not the case in our study. For validation of models it is therefore important that the number 
of events of the training and validation cohort match.

Some semantic features are lesion specific (e.g. cavitation and necrosis), while other semantic features describe lung characteristics (e.g. pleural effusion and satellite nodules). The univariable analysis clearly shows that tumor radiomic features contain information about tumor semantic features (e.g. density, air bronchogram, necrosis), while lung radiomic features contain information about lung semantic features (e.g.

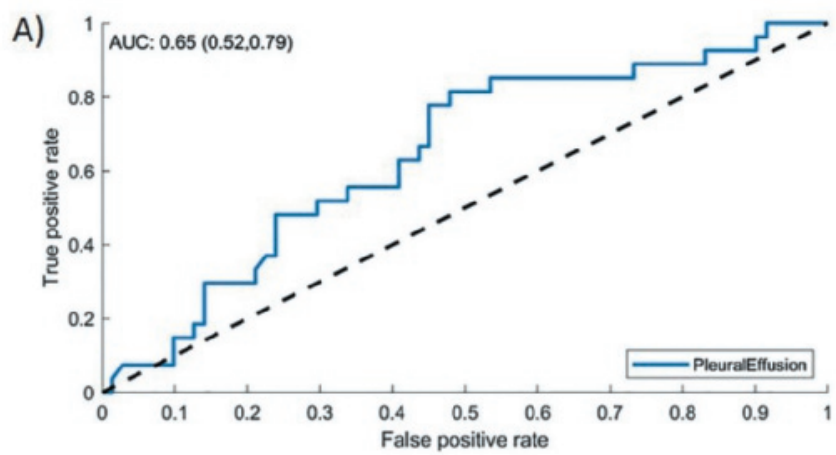

B)

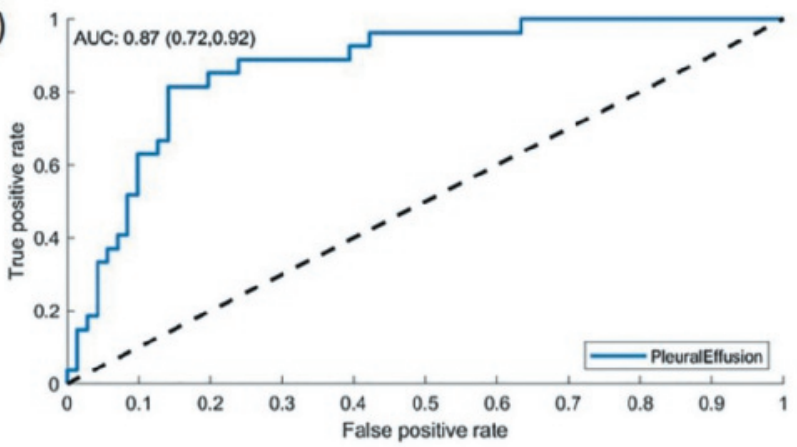

C)

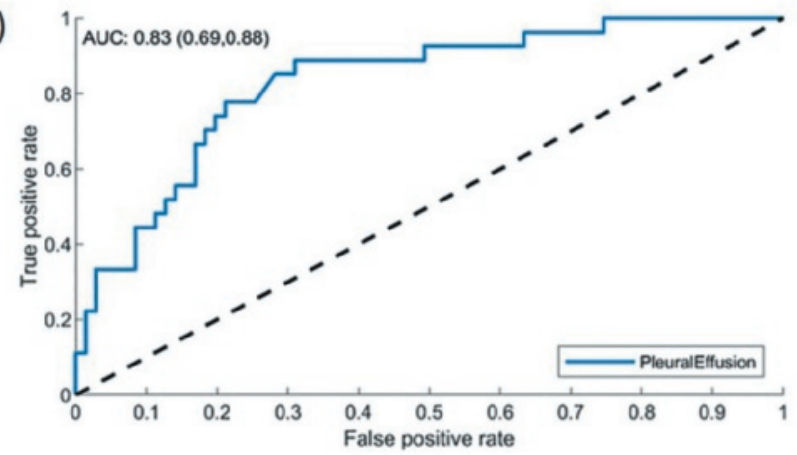

Figure 8.4: Example of the ROC curves of the validation dataset for pleural effusion with $A$ ) tumor radiomic features, B) lung radiomic features and C) combination of radiomic features. 
pleural contact and pleural effusion). No radiomic feature contained information to describe calcifications, but only a small proportion of $12 \%$ of the patients of the training cohort had calcifications and these calcifications were visually hard to detect, with the validation cohort only $3 \%$ showing calcifications. Furthermore, tailored radiomic features or other pre-processing techniques could improve detection of these semantic features.

The multivariable analysis showed that a combination of tumor radiomic features could detect the semantic features air bronchogram, necrosis and pleural effusion. A combination of lung radiomic features could detect the semantic features pleural thickening, satellite nodules and pleural effusion. This shows that there is an added value of combining radiomic features containing complementary information for prediction purposes.

The validation data in our study is part of a multicentric trial and therefore a large variability in image acquisition and reconstruction parameters exists. Although, we performed resampling of the voxel sizes, datasets with equal voxel sizes have more robust radiomics features [19]. This makes the semantic feature scoring as well as the radiomic features less reliable [20, 21]. Modelling semantic features using single center data is expected to result in higher AUC values. Phantom studies potentially lead to optimal post-processing settings and eventually correction factors, to overcome the variability of image acquisition in reconstruction settings in large datasets [19, 14, 22, 23].

A study of Liu et al. [24] showed that semantic features as well as radiomic features of the primary lung can predict pathological node involvement. A study of Wei et al. [25] showed that using semantic features malignancy of a lesion can be predicted. A study of Li et al. [16] used a combination of clinical variables, radiomic features and semantic features to predict recurrence after therapy. All these studies show that combining clinical variables, semantic features and radiomic features can be used to stratify patients.

For radiomic feature extraction the region of interest needs to be delineated/segmented, which is currently mostly done semi-automatically or manually. In our study the lungs are segmented using automatic atlas based segmentation, and manually adjusted if needed. This method was quite accurate and can therefore, in the future, easily be used for segmentation of large datasets [26]. Our study showed that some semantic features, for example necrosis, can be described by a combination of radiomic features, which by using automatic segmentation can reduce the inter-observer variability as well as the time factor. Deep learning in combination with radiomics overcomes this issue as well. A combination of deep learning and radiomics was shown to have predictive power which nodules are malignant [27].

To conclude, semantic features like pleural effusion and necrosis can be described using a single radiomic feature. Using a combination of radiomic features, the performance can be improved. While there are correlations between radiomic and semantic features, new approaches such as deep learning approaches with more training data are needed to describe better qualitiative semantic features. 


\section{References}

[1] K. M. Panth et al. "Is there a causal relationship between genetic changes and radiomics-based image features? An in vivo preclinical experiment with doxycycline inducible GADD34 tumor cells". In: Radiother Oncol 116.3 (2015). Radiotherapy and oncology : journal of the European Society for Therapeutic Radiology and Oncology, pp. 462-6. DOI: 10.1016/j.radonc.2015.06.013.

[2] H. Wang et al. "Semiquantitative Computed Tomography Characteristics for Lung Adenocarcinoma and Their Association With Lung Cancer Survival". In: Clin Lung Cancer 16.6 (2015). Clinical lung cancer, e141-63. DOI: 10.1016/j.cllc.2015.05.007.

[3] S. Novello et al. "Metastatic non-small-cell lung cancer: ESMO Clinical Practice Guidelines for diagnosis, treatment and follow-up". In: Ann Oncol 27.suppl 5 (2016). Annals of oncology : official journal of the European Society for Medical Oncology, pp. v1-v27. DOI: 10.1093/annonc/mdw326.

[4] S. Rizzo et al. "CT Radiogenomic Characterization of EGFR, K-RAS, and ALK Mutations in Non-Small Cell Lung Cancer". In: Eur Radiol 26.1 (2016). European radiology, pp. 32-42. DOI: 10.1007/s00330-015-3814-0.

[5] A. Onn et al. "Tumor cavitation in stage I non-small cell lung cancer: epidermal growth factor receptor expression and prediction of poor outcome". In: Radiology 237.1 (2005). Radiology, pp. 342-7. DOI: 10.1148/radiol.2371041650.

[6] S. Takashima et al. "Prognostic significance of high-resolution CT findings in small peripheral adenocarcinoma of the lung: a retrospective study on 64 patients". In: Lung Cancer 36.3 (2002). Lung cancer (Amsterdam, Netherlands), pp. 289-95.

[7] H. J. Aerts et al. "Decoding tumour phenotype by noninvasive imaging using a quantitative radiomics approach". In: Nat Commun 5 (2014). Nature communications, p. 4006. DOI: $10.1038 /$ ncomms5006.

[8] E. Rios Velazquez et al. "Somatic Mutations Drive Distinct Imaging Phenotypes in Lung Cancer". In: Cancer Res 77.14 (2017). Cancer research, pp. 3922-3930. DOI: 10.1158/0008-5472.can-17-0122.

[9] H. J. Yoon et al. "Decoding Tumor Phenotypes for ALK, ROS1, and RET Fusions in Lung Adenocarcinoma Using a Radiomics Approach". In: Medicine (Baltimore) 94.41 (2015). Medicine, e1753. DOI: 10.1097/md.0000000000001753.

[10] S. S. F. Yip et al. "Associations between radiologist-defined semantic and automatically computed radiomic features in non-small cell lung cancer". In: Sci Rep 7.1 (2017). Scientific reports, p. 3519. DOI: 10.1038/s41598-017-02425-5.

[11] Q. Li et al. "Imaging features from pretreatment CT scans are associated with clinical outcomes in nonsmall-cell lung cancer patients treated with stereotactic body radiotherapy". In: Med Phys 44.8 (2017). Medical physics, pp. 4341-4349. DOI: $10.1002 / \mathrm{mp} .12309$.

[12] C. Parmar et al. "Robust Radiomics feature quantification using semiautomatic volumetric segmentation." eng. In: PloS one 9 (7 2014), e102107.

[13] J. O. Deasy, A. I. Blanco, and V. H. Clark. "CERR: a computational environment for radiotherapy research". In: Med Phys 30.5 (2003). Medical physics, pp. 979-85. DOI: $10.1118 / 1.1568978$. 
[14] R. T. H. M. Larue et al. "Influence of gray level discretization on radiomic feature stability for different CT scanners, tube currents and slice thicknesses: a comprehensive phantom study". In: Acta Oncol In press (2017), p. 11. DOI: 10.1080/0284186X.2017.1351624.

[15] J. C. Peeken et al. "Semantic imaging features predict disease progression and survival in glioblastoma multiforme patients". In: Strahlenther Onkol 194.6 (2018). Strahlentherapie und Onkologie : Organ der Deutschen Rontgengesellschaft ... [et al], pp. 580-590. DOI: 10.1007/s00066-018-1276-4.

[16] Q. Li et al. "CT imaging features associated with recurrence in non-small cell lung cancer patients after stereotactic body radiotherapy". In: Radiat Oncol 12.1 (2017). Radiation oncology (London, England), p. 158. DOI: 10.1186/s13014-017-0892-y.

[17] J. E. van Timmeren et al. "Survival prediction of non-small cell lung cancer patients using radiomics analyses of cone-beam CT images". In: Radiother Oncol 123.3 (2017). Radiotherapy and oncology : journal of the European Society for Therapeutic Radiology and Oncology, pp. 363-369. DOI: 10.1016/j.radonc.2017.04.016.

[18] G. S. Collins, E. O. Ogundimu, and D. G. Altman. "Sample size considerations for the external validation of a multivariable prognostic model: a resampling study". In: Stat Med 35.2 (2016). Statistics in medicine, pp. 214-26. DOI: 10.1002/sim.6787.

[19] M. Shafiq-Ul-Hassan et al. "Intrinsic dependencies of CT radiomic features on voxel size and number of gray levels". In: Med Phys 44.3 (2017). Medical physics, pp. 1050-1062. DOI: $10.1002 / \mathrm{mp} .12123$.

[20] P. Lambin et al. "Radiomics: the bridge between medical imaging and personalized medicine". In: Nat Rev Clin Oncol 14.12 (2017). Nature reviews. Clinical oncology, pp. 749-762. DOI: $10.1038 /$ nrclinonc.2017.141.

[21] L. He et al. "Effects of contrast-enhancement, reconstruction slice thickness and convolution kernel on the diagnostic performance of radiomics signature in solitary pulmonary nodule". In: Sci Rep 6 (2016). Scientific reports, p. 34921. DOI: 10.1038/ srep34921.

[22] D. Mackin et al. "Measuring Computed Tomography Scanner Variability of Radiomics Features". In: Invest Radiol 50.11 (2015). Investigative radiology, pp. 757-65. DOI: $10.1097 /$ rli.0000000000000180.

[23] D. Mackin et al. "Harmonizing the pixel size in retrospective computed tomography radiomics studies". In: PLoS One 12.9 (2017). PloS one, e0178524. DOI: 10.1371/ journal.pone.0178524.

[24] Y. Liu et al. "Prediction of pathological nodal involvement by CT-based Radiomic features of the primary tumor in patients with clinically node-negative peripheral lung adenocarcinomas". In: Med Phys 45.6 (2018). Medical physics, pp. 2518-2526. DOI: $10.1002 / \mathrm{mp} .12901$.

[25] G. Wei et al. "Content-based image retrieval for Lung Nodule Classification Using Texture Features and Learned Distance Metric". In: J Med Syst 42.1 (2017). Journal of medical systems, p. 13. DOI: 10.1007/s10916-017-0874-5.

[26] B. Schipaanboord et al. "Can atlas-based auto-segmentation ever be perfect? Insights from Extreme Value Theory". In: IEEE Trans Med Imaging (2018). IEEE transactions on medical imaging. DOI: 10.1109/tmi.2018.2856464. 
[27] R. Paul et al. "Predicting malignant nodules by fusing deep features with classical radiomics features". In: J Med Imaging (Bellingham) 5.1 (2018). Journal of medical imaging (Bellingham, Wash.), p. 011021. DOI: 10.1117/1.jmi.5.1.011021. 


\section{Supplementary Material}

\section{Feature descriptions}

\section{Fractal Dimension features}

The Fractal Dimension (FD) of the image is computed as described by Al-Kadi and Watson (1). Given the FD processed image $I$, with $N$ elements:

1. Average

$$
\text { average }=\frac{1}{N} \sum I
$$

2. Lacunarity

$$
\text { lacunarity }=\frac{\frac{1}{N} \sum I^{2}}{\frac{1}{N^{2}} \sum I}-1
$$

3. Standard deviation

$$
\text { standard deviation }=\left(\frac{1}{N-1} \sum(I-\bar{I})^{2}\right)^{1 / 2}
$$

Where $\bar{I}$ is the mean of $I$. 


\section{First-order Grey-level statistics}

First-order Grey-level statistics describe the distribution of grey values within the volume. Let $X$ denote the three dimensional image matrix with $N$ voxels, $P$ the first order histogram, $P(i)$ the fraction of voxels with intensity level $i$ and $N_{l}$ the number of discrete intensity levels.

\section{Energy}

$$
\text { energy }=\sum_{i=1}^{N} X(i)^{2}
$$

Energy is also known as the sum of squares.

\section{Entropy}

$$
\text { entropy }=\sum_{i=1}^{N_{l}} P(i) \log _{2} P(i)
$$

\section{Kurtosis}

$$
\text { kurtosis }=\frac{\frac{1}{N} \sum_{i=1}^{N}(X(i)-\bar{X})^{4}}{\left(\frac{1}{N} \sum_{i=1}^{N}(X(i)-\bar{X})^{2}\right)^{2}}
$$

where $\bar{X}$ is the mean of $X$.

\section{Maximum}

The maximum grey value of $X$.

$$
\text { maximum }=\max (X)
$$

\section{Mean}

The mean grey value of $X$.

$$
\text { mean }=\frac{1}{N} \sum_{i=1}^{N} X(i)
$$

\section{Mean absolute deviation}

The mean of the absolute deviations of all voxel intensities around the mean intensity value. 


$$
\text { mean absolute deviation }=\frac{1}{N} \sum_{i=1}^{N}|X(i)-\bar{X}|
$$

where $\bar{X}$ is the mean of $X$.

\section{Median}

The sample median of $X$, or the $50^{\text {th }}$ percentile of $X$.

\section{Minimum}

The minimum intensity value of $X$.

$$
\text { minimum }=\min (X)
$$

\section{Range}

The range of intensity values of $X$.

$$
\text { range }=\max (X)-\min (X)
$$

\section{Root mean square (RMS)}

The quadratic mean, or the square root of the mean of squares of all voxel intensities.

$$
R M S=\sqrt{\frac{\sum_{i}^{N} X(i)^{2}}{N}}
$$

14. Skewness

$$
\text { skewness }=\frac{\frac{1}{N} \sum_{i=1}^{N}(X(i)-\bar{X})^{3}}{\left(\sqrt{\frac{1}{N} \sum_{i=1}^{N}(X(i)-\bar{X})^{2}}\right)^{3}}
$$

where $\bar{X}$ is the mean of $X$.

\section{Standard deviation}

$$
\text { standard deviation }=\left(\frac{1}{N-1} \sum_{i=1}^{N}(X(i)-\bar{X})^{2}\right)^{1 / 2}
$$

where $\bar{X}$ is the mean of $X$. 


\section{Robust mean absolute deviation}

The mean absolute deviation (0) of only those voxels in $X$ with a grey value between the $10^{\text {th }}$ and $90^{\text {th }}$ percentile.

\section{7. $10^{\text {th }}$ percentile}

The $10^{\text {th }}$ percentile of $X$, a robust alternative to the minimum grey value (11).

\section{8. $90^{\text {th }}$ percentile}

The $90^{\text {th }}$ percentile of $X$, a robust alternative to the maximum grey value (7).

\section{Interquartile range}

The interquartile range is defined as the $75^{\text {th }}$ minus the $25^{\text {th }}$ percentile of $X$.

\section{Uniformity}

$$
\text { uniformity }=\sum_{i=1}^{N_{l}} P(i)^{2}
$$

\section{Variance}

$$
\text { variance }=\frac{1}{N-1} \sum_{i=1}^{N}(X(i)-\bar{X})^{2}
$$

where $\bar{X}$ is the mean of $X$. Variance is the square of the standard deviation (15). 


\section{Intensity histogram features}

Intensity histogram features describe the distribution of grey values within the volume, after discretization into intensity level bins was applied. Let:

$X_{d}=\left\{X_{d, 1}, X_{d, 2}, \ldots, X_{d, N_{v}}\right\}$ be the set of discretized intensity values of the $N_{v}$ voxels in the volume of interest,

$H=\left\{n_{1}, n_{2}, \ldots\right\}$ be the histogram with frequency count $n_{i}$ of each discretized intensity level $i$ in $X_{d}$ $N_{g}$ be the number of discretized intensity values (bins) in the volume of interest,

$p_{i}$ be the occurrence probability for each bin $i$ of the histogram $N_{g}$, where $p_{i}=n_{i} / N_{v}$.

22. Coefficient of variance (cov)

$$
\operatorname{cov}=\frac{\text { standard deviation }}{\text { mean }}
$$

23. Energy

$$
\text { energy }=\sum_{j=1}^{N_{v}} X_{d}(j)^{2}
$$

Energy is also known as the sum of squares.

\section{Entropy}

$$
\text { entropy }=-\sum_{i=1}^{N_{g}} P(i) \log _{2} P(i)
$$

25. Interquartile range (iqr)

$$
I Q R=P_{75}-P_{25}
$$

where $P_{25}$ and $P_{75}$ are the $25^{\text {th }}$ and $75^{\text {th }}$ percentile of $X_{d}$, respectively.

\section{Kurtosis}

$$
\text { kurtosis }=\frac{\frac{1}{N_{v}} \sum_{j=1}^{N_{v}}\left(X_{d}(j)-\bar{X}_{d}\right)^{4}}{\left(\frac{1}{N_{v}} \sum_{j=1}^{N_{v}}\left(X_{d}(j)-\bar{X}_{d}\right)^{2}\right)^{2}}
$$

where $\bar{X}_{d}$ is the mean of $X_{d}$. 


\section{Maximum}

The maximum discretized intensity value of $X_{d}$.

$$
\text { maximum }=\max \left(X_{d}\right)
$$

\section{Maximum histogram gradient (maxgrad)}

$$
\text { maxgrad }=\max \left(H^{\prime}\right)
$$

Where $H^{\prime}$ is the histogram gradient, defined as:

$$
H^{\prime}=\left\{H(2)-H(1), \ldots, \frac{H(i+1)-H(i-1)}{2}, \ldots, H\left(N_{g}\right)-H\left(N_{g}-1\right)\right\}
$$

\section{Maximum histogram gradient intensity level (maxgradi)}

The discretized intensity level $i$ corresponding to the maximum histogram gradient.

\section{Mean}

The mean discretized intensity value of $X_{d}$.

$$
\text { mean }=\frac{1}{N_{v}} \sum_{j=1}^{N_{v}} X_{d}(j)
$$

\section{Mean absolute deviation (meand)}

The mean of the absolute deviations of all discretized intensity levels around the mean of $X_{d}$.

$$
\text { meand }=\frac{1}{N_{v}} \sum_{j=1}^{N_{v}}\left|X_{d}(j)-\overline{X_{d}}\right|
$$

where $\bar{X}_{d}$ is the mean of $X_{d}$.

\section{Median}

The sample median of $X_{d}$ or the $50^{\text {th }}$ percentile of $X_{d}$.

\section{Median absolute deviation (mediand)}

The dispersion from the median of $X_{d}$.

$$
\text { mediand }=\frac{1}{N_{v}} \sum_{j=1}^{N_{v}}\left|X_{d}(j)-M\right|
$$


where $M$ is the median of $X_{d}$.

\section{Minimum}

The minimum discretized intensity value of $X_{d}$.

$$
\text { minimum }=\min \left(X_{d}\right)
$$

35. Minimum histogram gradient (mingrad)

$$
\text { mingrad }=\min \left(H^{\prime}\right)
$$

Where $H^{\prime}$ is the histogram gradient, defined as:

$$
H^{\prime}=\left\{H(2)-H(1), \ldots, \frac{H(i+1)-H(i-1)}{2}, \ldots, H\left(N_{g}\right)-H\left(N_{g}-1\right)\right\}
$$

\section{Minimum histogram gradient intensity level (mingradi)}

The discretized intensity level $i$ corresponding to the minimum histogram gradient.

\section{Mode}

The mode of $X_{d}$ is the most frequently occurring discretized image level present. In case multiple bins have the highest count $n_{i}$, the mode is the smallest of those values.

\section{Uniformity}

$$
\text { uniformity }=\sum_{i=1}^{N_{g}} P(i)^{2}
$$

\section{Range}

The range of bins in the histogram, i.e. the width of the histogram.

$$
\text { range }=\max \left(X_{d}\right)-\min \left(X_{d}\right)
$$

40. Root mean square (RMS):

$$
R M S=\sqrt{\frac{\sum_{j=1}^{N_{v}} X_{d}(j)^{2}}{N_{v}}}
$$




\section{Robust mean absolute deviation (rmeand)}

Similar to mean absolute deviation, but in this case only considering the set of intensity levels in the range between the $10^{\text {th }}$ and $90^{\text {th }}$ percentile of $X_{d}$.

$$
\text { rmeand }=\frac{1}{N_{10-90}} \sum_{j=1}^{N_{10-90}}\left|X_{d, 10-90}(j)-\bar{X}_{d, 10-90}\right|
$$

where $X_{10-90}$ represents the set of $N_{10-90}$ voxels in $X_{d}$ whose discretized intensity levels fall within the range of the $10^{\text {th }}$ till the $90^{\text {th }}$ percentile of $X_{d}$.

42. Skewness

$$
\text { skewness }=\frac{\frac{1}{N_{v}} \sum_{j=1}^{N_{v}}\left(X_{d}(j)-\bar{X}_{d}\right)^{3}}{\left(\sqrt{\frac{1}{N_{v}} \sum_{j=1}^{N_{v}}\left(X_{d}(j)-\bar{X}_{d}\right)^{2}}\right)^{3}}
$$

\section{Standard deviation}

$$
\text { standard deviation }=\left(\frac{1}{N_{v}-1} \sum_{j=1}^{N_{v}}\left(X_{d}(j)-\bar{X}_{d}\right)^{2}\right)^{1 / 2}
$$

\section{Variance}

The variance of $X_{d}$.

$$
\text { variance }=\frac{1}{N_{v}-1} \sum_{j=1}^{N_{v}}\left(X_{d}(j)-\overline{X_{d}}\right)^{2}
$$

where $\overline{X_{d}}$ is the mean of $X_{d}$.

\section{5. $10^{\text {th }}$ percentile}

The $10^{\text {th }}$ percentile of $X_{d}$.

\section{6. $90^{\text {th }}$ percentile}

The $90^{\text {th }}$ percentile of $X_{d}$. 


\section{Quartile coefficient of dispersion (qcod)}

The quartile coefficient of dispersion is a robust alternative to the coefficient of variance.

$$
q \operatorname{cod}=\frac{P_{75}-P_{25}}{P_{75}+P_{25}}
$$

where $P_{25}$ and $P_{75}$ are the $25^{\text {th }}$ and $75^{\text {th }}$ percentile of $X_{d}$, respectively. 


\section{Local Intensity features}

Local Intensity (LocInt) features are defined based on local intensity values around a center voxel (2).

\section{Local intensity peak}

Mean intensity level in a $1 \mathrm{~cm}^{3}$ spherical volume, centered on the voxel with the maximum intensity level in the volume of interest. In case multiple voxels contain the maximum intensity level, the highest mean intensity level of all spherical volumes is used.

\section{Global Intensity peak}

Similar to local intensity peak, but in this case the mean intensity level in a $1 \mathrm{~cm}^{3}$ spherical volume is calculated for every voxel in the volume of interest. The highest mean intensity level of all spherical volumes is selected as the global intensity peak feature. 


\section{Geometric features}

Geometric features describe the shape and size of the volume of interest. Let $V$ be the volume and $A$ the surface area of the volume of interest. Let $N$ be the total number of voxels, $X=\left\{\vec{X}_{1}, \vec{X}_{2}, \ldots, \vec{X}_{N}\right\}$ the set of $\mathrm{N}$ Cartesian coordinate vectors and $I=\left\{I_{1}, I_{2}, \ldots, I_{N}\right\}$ the corresponding intensity values.

\section{Asphericity}

$$
\text { asphericity }=\left(\frac{1}{36 \pi} \frac{A^{3}}{V^{2}}\right)^{\frac{1}{3}}-1
$$

\section{Centroid distance}

The centroid distance is the Euclidean distance between the geometric centroid $\left(C_{g}\right)$ and the centroid weighing each voxel by its intensity value $\left(C_{i}\right)$. The centroid distance is a measure of how close the high intensity values are to the geometric center.

$$
\begin{gathered}
C_{g}=\frac{1}{N} \sum_{i=1}^{N} \vec{X}_{i} \\
C_{i}=\frac{\sum_{i=1}^{N} I \vec{X}_{i}}{\sum_{i=1}^{N} I} \\
\text { centroid distance }=\left\|C_{g}-C_{i}\right\|
\end{gathered}
$$

\section{Compactness 1}

Compactness is a measure of how much the volume resembles a sphere, as described by Aerts et al. (3).

$$
\text { compactness } 1=\frac{V}{\sqrt{\pi} A^{\frac{2}{3}}}
$$

53. Compactness 2

$$
\text { compactness } 2=36 \pi \frac{V^{2}}{A^{3}}
$$




\section{Compactness 3}

$$
\text { compactness } 3=\frac{V}{\sqrt{\pi} A^{\frac{3}{2}}}
$$

A dimensionless alternative to Compactness (52), as described by Aerts et al. (3).

\section{Maximum diameter}

The maximum diameter is the largest pairwise difference between voxels on the surface of the volume, in $3 \mathrm{D}$ and for each plane separately. The following diameters are calculated:
a. The maximum three-dimensional tumor diameter.
b. The maximum two-dimensional diameter of all transversal planes.
c. The maximum two-dimensional diameter of all sagittal planes.
d. The maximum two-dimensional diameter of all coronal planes.

\section{Major axis length}

Axis lengths are measures of the extent of the volume along its three principle axis. Principle component analysis (PCA) on the $x, y$ and $z$ coordinates of all voxels within the volume is used to determine the three orthogonal eigenvectors and corresponding eigenvalues $\left(\lambda_{\text {max }}, \lambda_{\text {minor }}, \lambda_{\text {min }}\right)$. The major axis length is the largest eigenvalue $\left(\lambda_{\max }\right)$ as determined by PCA.

\section{Minor axis length}

The largest eigenvalue $\left(\lambda_{\text {minor }}\right)$ as determined by PCA.

\section{Least axis length}

The smallest eigenvalue $\left(\lambda_{\min }\right)$ as determined by PCA.

\section{Elongation}

$$
\text { elongation }=\frac{\lambda_{\text {minor }}}{\lambda_{\max }}
$$

\section{Flatness}

$$
\text { flatness }=\frac{\lambda_{\min }}{\lambda_{\max }}
$$




\section{Spherical disproportion (4)}

Spherical disproportion is a measure of how much the volume resembles a sphere.

$$
\text { spherical disproportion }=\frac{A}{4 \pi R^{2}}
$$

Where $A$ is the surface area and $R$ is the radius of a sphere with the same volume as the tumor, obtained through:

$$
R=\sqrt[3]{\frac{3 V}{4 \pi}}
$$

\section{Sphericity (4)}

Sphericity is a measure of how much the volume resembles a sphere.

$$
\text { sphericity }=\frac{\pi^{\frac{1}{3}}(6 V)^{\frac{2}{3}}}{A}=\frac{\left(36 \pi V^{2}\right)^{\frac{1}{3}}}{A}
$$

\section{Surface area}

The surface area is calculated by triangulation (i.e. dividing the surface into connected triangles, which define the isosurface enclosing the volume) and is defined as:

$$
\text { surface area }=\sum_{i=1}^{N} \frac{1}{2}\left|a_{i} b_{i} \times a_{i} c_{i}\right|
$$

Where $N$ is the total number of triangles covering the surface and $a, b$ and $c$ are edge vectors of the triangles.

\section{Surface to volume ratio}

$$
\text { surface to volume ratio }=\frac{A}{V}
$$

\section{Volume}

The volume is defined as the number of voxels within the volume multiplied by the voxel volume.

$$
\text { volume }=N v
$$

Where $v$ is the volume of a single voxel. 


\section{Grey-Level Co-Occurrence Matrix based features}

Grey-level co-occurrence matrix (GLCM) based features, as originally described by Haralick et al (5). A normalized GLCM is defined as $P(i, j ; \delta, \alpha)$, a matrix with size $N_{g} \times N_{g}$ describing the second-order joint probability function of an image, where the $(i, j)$ th element represents the number of times the combination of intensity levels $i$ and $j$ occur in two pixels in the image, that are separated by a distance of $\delta$ pixels in direction $\alpha$, and $N_{g}$ is the maximum discrete intensity level in the image. Let:

$P(i, j)$ be the normalized (i.e. $\sum P(i, j)=1$ ) co-occurrence matrix, generalized for any $\delta$ and $\alpha$,

$p_{x}(i)=\sum_{j=1}^{N_{g}} P(i, j)$,

$p_{y}(j)=\sum_{i=1}^{N_{g}} P(i, j)$,

$\mu_{x}$ be the mean of $p_{x}$, where $\mu_{x}=\sum_{i=1}^{N_{g}} \sum_{j=1}^{N_{g}} i P(i, j)$

$\mu_{y}$ be the mean of $p_{y}$, where $\mu_{y}=\sum_{i=1}^{N_{g}} \sum_{j=1}^{N_{g}} j P(i, j)$

$\sigma_{x}$ be the standard deviation of $p_{x}$, where $\sigma_{x}^{2}=\sum_{i=1}^{N_{g}} \sum_{j=1}^{N_{g}} P(i, j)\left(i-\mu_{x}\right)^{2}$

$\sigma_{y}$ be the standard deviation of $p_{y}$, where $\sigma_{y}^{2}=\sum_{i=1}^{N_{g}} \sum_{j=1}^{N_{g}} P(i, j)\left(j-\mu_{y}\right)^{2}$

$p_{x+y}(k)=\sum_{i=1}^{N_{g}} \sum_{j=1}^{N_{g}} P(i, j), i+j=k, k=2,3, \ldots, 2 N_{g}$,

$p_{x-y}(k)=\sum_{i=1}^{N_{g}} \sum_{j=1}^{N_{g}} P(i, j),|i-j|=k, k=0,1, \ldots, N_{g}-1$,

$H X Y 1=-\sum_{i=1}^{N_{g}} \sum_{j=1}^{N_{g}} P(i, j) \ln \left(p_{x}(i) p_{y}(j)\right)$,

$H X Y 2=-\sum_{i=1}^{N_{g}} \sum_{j=1}^{N_{g}} p_{x}(i) p_{y}(j) \ln \left(p_{x}(i) p_{y}(j)\right)$

$H X=-\sum p_{x} \ln \left(p_{x}\right)$

$H Y=-\sum p_{y} \ln \left(p_{y}\right)$

\section{Average $(\mu)$}

$$
\text { average }(\mu)=\frac{\sum_{i=1}^{N_{g}} \sum_{j=1}^{N_{g}}(i+j) P(i, j)}{2}
$$

Note that for a symmetrical GLCM, $\mu=\mu_{x}=\mu_{y}$.

\section{Autocorrelation}

$$
\text { autocorrelation }=\sum_{i=1}^{N_{g}} \sum_{j=1}^{N_{g}} i j P(i, j)
$$


68. Cluster Prominence

$$
\text { cluster prominence }=\sum_{i=1}^{N_{g}} \sum_{j=1}^{N_{g}}\left[i+j-\mu_{x}-\mu_{y}\right]^{4} P(i, j)
$$

69. Cluster Shade

$$
\text { cluster shade }=\sum_{i=1}^{N_{g}} \sum_{j=1}^{N_{g}}\left[i+j-\mu_{x}-\mu_{y}\right]^{3} P(i, j)
$$

70. Cluster Tendency

$$
\text { cluster tendency }=\sum_{i=1}^{N_{g}} \sum_{j=1}^{N_{g}}\left[i+j-\mu_{x}-\mu_{y}\right]^{2} P(i, j)
$$

71. Contrast (6)

$$
\text { contrast }=\sum_{i=1}^{N_{g}} \sum_{j=1}^{N_{g}}|i-j|^{2} P(i, j)=\sum_{k=0}^{N_{g}-1} k^{2} p_{x-y}(k)
$$

72. Correlation

$$
\text { correlation }=\frac{\sum_{i=1}^{N_{g}} \sum_{j=1}^{N_{g}} i j P(i, j)-\mu_{x} \mu_{y}}{\sigma_{x} \sigma_{y}}
$$

73. Difference Average $\left(\mu_{x-y}\right)$

$$
\text { difference average }\left(\mu_{x-y}\right)=\sum_{k=0}^{N_{g}-1} k p_{x-y}
$$

74. Difference Entropy

$$
\text { difference entropy }=-\sum_{i=0}^{N_{g}-1} P_{x-y}(i) \log _{2}\left[P_{x-y}(i)\right]
$$




\section{Difference Variance}

$$
\text { difference variance }=\sum_{i=0}^{N_{g}-1}\left(i-\mu_{x-y}\right)^{2} P_{x-y}(i)
$$

76. Dissimilarity

$$
\text { dissimilarity }=\sum_{i=1}^{N_{g}} \sum_{j=1}^{N_{g}}|i-j| P(i, j)
$$

77. Energy (7)

$$
\text { energy }=\sum_{i=1}^{N_{g}} \sum_{j=1}^{N_{g}}[P(i, j)]^{2}
$$

This feature is also called Angular Second Moment (ASM) and Uniformity (6).

\section{Entropy (H)}

$$
\operatorname{entropy}(H)=-\sum_{i=1}^{N_{g}} \sum_{j=1}^{N_{g}} P(i, j) \log _{2}[P(i, j)]
$$

\section{Homogeneity 1}

$$
\text { homogeneity } 1=\sum_{i=1}^{N_{g}} \sum_{j=1}^{N_{g}} \frac{P(i, j)}{1+|i-j|}
$$

This feature is also called Inverse Difference (6).

80. Homogeneity 2 (7)

$$
\text { homogeneity } 2=\sum_{i=1}^{N_{g}} \sum_{j=1}^{N_{g}} \frac{P(i, j)}{1+|i-j|^{2}}
$$

This feature is also called Inverse Difference Moment (6). 
81. Informational measure of correlation 1 (IMC1)

$$
I M C 1=\frac{H-H X Y 1}{\max \{H X, H Y\}}
$$

Where $H$ is the entropy (78).

82. Informational measure of correlation 2 (IMC2)

$$
I M C 2=\sqrt{1-e^{-2(H X Y 2-H)}}
$$

Where $H$ is the entropy (78).

83. Inverse Difference Moment Normalized (IDMN)

$$
I D M N=\sum_{i=1}^{N_{g}} \sum_{j=1}^{N_{g}} \frac{P(i, j)}{1+\left(\frac{|i-j|^{2}}{N_{g}^{2}}\right)}
$$

84. Inverse Difference Normalized (IDN)

$$
I D N=\sum_{i=1}^{N_{g}} \sum_{j=1}^{N_{g}} \frac{P(i, j)}{1+\left(\frac{|i-j|}{N_{g}}\right)}
$$

85. Inverse variance

$$
\text { inverse variance }=\sum_{i=1}^{N_{g}} \sum_{j=1}^{N_{g}} \frac{P(i, j)}{|i-j|^{2}} \quad, i \neq j
$$

\section{Maximal Correlation Coefficient}

$$
\begin{aligned}
& \text { maximal correlation coefficient }=\sqrt{\text { second largest eigenvalue of } Q} \\
& \qquad Q=\sum_{k=1}^{N_{g}} \frac{P(i, k) P(j, k)}{p_{x}(i) p_{y}(k)}
\end{aligned}
$$

87. Maximum Probability

$$
\text { maximum probability }=\max \{P(i, j)\}
$$


88. Sum average (SA)

$$
\text { sum average }(S A)=\sum_{i=2}^{2 N_{g}}\left[i P_{x+y}(i)\right]
$$

89. Sum entropy

$$
\text { sum entropy }=-\sum_{i=2}^{2 N_{g}} P_{x+y}(i) \log _{2}\left[P_{x+y}(i)\right]
$$

90. Sum variance

$$
\text { sum variance }=\sum_{i=2}^{2 N_{g}}(i-S A)^{2} P_{x+y}(i)
$$

91. Variance (sum of squares)

$$
\text { variance }=\sum_{i=1}^{N_{g}} \sum_{j=1}^{N_{g}}(i-\mu)^{2} P(i, j)
$$




\section{Grey-Level Run-Length matrix based features}

Grey-level run-length matrix (GLRLM) based features, as described by Galloway et al. (8). Run length metrics quantify grey level runs in an image. A grey level run is defined as the length in number of pixels, of consecutive pixels that have the same grey level value. In a grey level run length matrix $p(i, j \mid \theta)$, the $(i, j)$ th element describes the number of times $j$ a grey level $i$ appears consecutively in the direction specified by $\theta$. Let:

$p(i, j)$ be the $(i, j)$ th entry in the given run-length matrix $p$, generalized for any direction $\theta$,

$N_{g}$ the number of discrete intensity values in the image,

$N_{r}$ the maximum run length,

$N_{S}$ the total number of runs, where $N_{S}=\sum_{i=1}^{N_{g}} \sum_{j=1}^{N_{r}} p(i, j)$,

$p_{r}$ the sum distribution of the number of runs with run length $j$, where $p_{r}(j)=\sum_{i=1}^{N_{g}} p(i, j)$,

$p_{g}$ the sum distribution of the number of runs with grey level $i$, where $p_{g}(i)=\sum_{j=1}^{N_{r}} p(i, j)$,

$N_{p}$ the number of voxels in the image, where $N_{p}=\sum_{j=1}^{N_{r}} j p_{r}$,

$p_{n}(i, j)$ the normalized run-length matrix, where $p_{n}(i, j)=\frac{p(i, j)}{N_{s}}$,

$\mu_{r}$ the mean run length, where $\mu_{r}=\sum_{i=1}^{N_{g}} \sum_{j=1}^{N_{r}} j p_{n}(i, j)$,

$\mu_{g}$ the mean grey level, where $\mu_{g}=\sum_{i=1}^{N_{g}} \sum_{j=1}^{N_{r}} i p_{n}(i, j)$.

92. Short Run Emphasis (SRE)

$$
S R E=\frac{1}{N_{S}} \sum_{j=1}^{N_{r}} \frac{p_{r}}{j^{2}}
$$

93. Long Run Emphasis (LRE)

$$
L R E=\frac{1}{N_{s}} \sum_{j=1}^{N_{r}} j^{2} p_{r}
$$

94. Grey Level Non-Uniformity (GLN)

$$
G L N=\frac{1}{N_{s}} \sum_{i=1}^{N_{g}} p_{g}^{2}
$$


95. Grey Level Non-Uniformity Normalized (GLNN)

$$
G L N N=\frac{1}{N_{S}^{2}} \sum_{i=1}^{N_{g}} p_{g}^{2}
$$

96. Run Length Non-Uniformity (RLN)

$$
R L N=\frac{1}{N_{s}} \sum_{j=1}^{N_{r}} p_{r}^{2}
$$

97. Run Length Non-Uniformity Normalized (RLNN)

$$
R L N N=\frac{1}{N_{S}^{2}} \sum_{j=1}^{N_{r}} p_{r}^{2}
$$

98. Run Percentage (RP)

$$
R P=\frac{N_{s}}{N_{p}}
$$

99. Low Grey Level Run Emphasis (LGRE)

$$
L G R E=\frac{1}{N_{s}} \sum_{i=1}^{N_{g}} \frac{p_{g}}{i^{2}}
$$

100. High Grey Level Run Emphasis (HGRE)

$$
H G R E=\frac{1}{N_{s}} \sum_{i=1}^{N_{g}} i^{2} p_{g}
$$

101. Short Run Low Grey Level Emphasis (SRLGE)

$$
S R L G E=\frac{1}{N_{S}} \sum_{i=1}^{N_{g}} \sum_{j=1}^{N_{r}} \frac{p(i, j)}{i^{2} j^{2}}
$$


102. Short Run High Grey Level Emphasis (SRHGE)

$$
S R H G E=\frac{1}{N_{S}} \sum_{i=1}^{N_{g}} \sum_{j=1}^{N_{r}} \frac{p(i, j) i^{2}}{j^{2}}
$$

103. Long Run Low Grey Level Emphasis (LRLGE)

$$
L R L G E=\frac{1}{N_{s}} \sum_{i=1}^{N_{g}} \sum_{j=1}^{N_{r}} \frac{p(i, j) j^{2}}{i^{2}}
$$

104. Long Run High Grey Level Emphasis (LRHGE)

$$
\text { LRHGE }=\frac{1}{N_{s}} \sum_{i=1}^{N_{g}} \sum_{j=1}^{N_{r}} p(i, j) i^{2} j^{2}
$$

105. Grey level variance (GLV)

$$
G L V=\sum_{i=1}^{N_{g}} \sum_{j=1}^{N_{r}}\left(i-\mu_{g}\right)^{2} p_{n}(i, j)
$$

106. Run length variance (RLV)

$$
R L V=\sum_{i=1}^{N_{g}} \sum_{j=1}^{N_{r}}\left(j-\mu_{r}\right)^{2} p_{n}(i, j)
$$

107. Run entropy (RE) (9)

$$
R E=-\sum_{i=1}^{N_{g}} \sum_{j=1}^{N_{r}} p_{n}(i, j \mid \theta) \log _{2}\left[p_{n}(i, j)\right]
$$




\section{Grey-Level size-zone matrix based features}

Grey-level size-zone matrix (GLSZM) based features, as described by Thibault et al. $(10,11)$. A grey level size-zone matrix describes the amount of homogeneous connected areas within the volume, of a certain size and intensity. The $(i, j)$ th entry of the $\operatorname{GLSZM} p(i, j)$ is the number of connected areas of grey level (i.e. intensity value) $i$ and size $j$. GLSZM features therefore describe homogeneous areas within the tumor volume, describing tumor heterogeneity at a regional scale (12). Let:

$p(i, j)$ be the $(i, j)$ th entry in the given GLSZM $p$,

$N_{g}$ the number of discrete intensity values in the image,

$N_{Z}$ the size of the largest, homogeneous region in the volume of interest,

$N_{S}$ the total number of homogeneous regions (zones), where $N_{S}=\sum_{i=1}^{N_{g}} \sum_{j=1}^{N_{z}} p(i, j)$,

$p_{z}$ the sum distribution of the number of zones with size $j$, where $p_{z}(j)=\sum_{i=1}^{N_{g}} p(i, j)$,

$p_{g}$ the sum distribution of the number of zones with grey level $i$, where $p_{g}(i)=\sum_{j=1}^{N_{z}} p(i, j)$,

$N_{p}$ the number of voxels in the image, where $N_{p}=\sum_{j=1}^{N_{z}} j p_{z}$,

$p_{n}(i, j)$ the normalized size-zone matrix, where $p_{n}(i, j)=\frac{p(i, j)}{N_{s}}$,

$\mu_{z}$ the mean zone size, where $\mu_{z}=\sum_{i=1}^{N_{g}} \sum_{j=1}^{N_{z}} j p_{n}(i, j \mid \theta)$,

$\mu_{g}$ the mean grey level, where $\mu_{g}=\sum_{i=1}^{N_{g}} \sum_{j=1}^{N_{z}} i p_{n}(i, j \mid \theta)$.

108. Small area Emphasis (SAE)

$$
S A E=\frac{1}{N_{S}} \sum_{j=1}^{N_{z}} \frac{p_{z}}{j^{2}}
$$

109. Large area Emphasis (LAE)

$$
L A E=\frac{1}{N_{s}} \sum_{j=1}^{N_{z}} j^{2} p_{z}
$$

110. Intensity Non-Uniformity (IN)

$$
I N=\frac{1}{N_{s}} \sum_{i=1}^{N_{g}} p_{g}^{2}
$$


111. Intensity Non-Uniformity Normalized (INN)

$$
I N N=\frac{1}{N_{S}^{2}} \sum_{i=1}^{N_{g}} p_{g}^{2}
$$

112. Size-zone Non-Uniformity (SZN)

$$
S Z N=\frac{1}{N_{S}} \sum_{j=1}^{N_{Z}} p_{Z}^{2}
$$

113. Size-zone Non-Uniformity Normalized (SZNN)

$$
S Z N N=\frac{1}{N_{S}^{2}} \sum_{j=1}^{N_{Z}} p_{Z}^{2}
$$

114. Zone Percentage (ZP)

$$
Z P=\frac{N_{s}}{N_{p}}
$$

115. Low intensity Emphasis (LIE)

$$
L I E=\frac{1}{N_{s}} \sum_{i=1}^{N_{g}} \frac{p_{g}}{i^{2}}
$$

116. High intensity Emphasis (HIE)

$$
H I E=\frac{1}{N_{S}} \sum_{i=1}^{N_{g}} i^{2} p_{g}
$$

117. Low intensity small area Emphasis (LISAE)

$$
\text { LISAE }=\frac{1}{N_{S}} \sum_{i=1}^{N_{g}} \sum_{j=1}^{N_{z}} \frac{p(i, j)}{i^{2} j^{2}}
$$


118. High intensity small area Emphasis (HISAE)

$$
H I S A E=\frac{1}{N_{S}} \sum_{i=1}^{N_{g}} \sum_{j=1}^{N_{z}} \frac{p(i, j) i^{2}}{j^{2}}
$$

119. Low intensity large area Emphasis (LILAE)

$$
\text { LILAE }=\frac{1}{N_{S}} \sum_{i=1}^{N_{g}} \sum_{j=1}^{N_{z}} \frac{p(i, j) j^{2}}{i^{2}}
$$

120. High intensity large area Emphasis (HILAE)

$$
\text { HILAE }=\frac{1}{N_{s}} \sum_{i=1}^{N_{g}} \sum_{j=1}^{N_{z}} p(i, j) i^{2} j^{2}
$$

121. Intensity variance (IV)

$$
I V=\sum_{i=1}^{N_{g}} \sum_{j=1}^{N_{z}}\left(i-\mu_{g}\right)^{2} p_{n}(i, j)
$$

122. Size-zone variance (SZV)

$$
S Z V=\sum_{i=1}^{N_{g}} \sum_{j=1}^{N_{Z}}\left(j-\mu_{z}\right)^{2} p_{n}(i, j)
$$

123. Zone entropy (ZE)

$$
Z E=\sum_{i=1}^{N_{g}} \sum_{j=1}^{N_{Z}} p_{n}(i, j) \log _{2}\left[p_{n}(i, j)\right]
$$




\section{Grey-Level distance-zone matrix based features}

Grey-level distance-zone matrix (GLDZM) based features, as described by Thibault et al. (13). A grey level distance-zone matrix describes the amount of homogeneous connected areas within the volume, of a certain intensity and distance to the shape border. The shape border is defined by 6 -connectedness in 3D (i.e. a voxel is on the border, if at least one face is exposed). In contrast to the original definition by Thibault et al. (13), the minimum distance to the border is 1 , instead of 0 (i.e. voxels on the border have a distance of 1$)$, to allow for correct feature calculations. The $(i, j)$ th entry of the $\operatorname{GLDM} p(i, j)$ is the number of connected areas of grey level (i.e. intensity value) $i$ and minimum distance $j$ to the shape border. GLSZM features therefore describe the radial distribution of homogeneous areas within the tumor volume. Let: $p(i, j)$ be the $(i, j)$ th entry in the given GLDZM $p$,

$N_{g}$ the number of discrete intensity values in the image,

$N_{d}$ the largest distance of a homogeneous region in the volume of interest to the shape border,

$N_{s}$ the total number of homogeneous regions (zones), where $N_{s}=\sum_{i=1}^{N_{g}} \sum_{j=1}^{N_{d}} p(i, j)$,

$p_{d}$ the sum distribution of the number of zones with distance $j$, where $p_{z}(j)=\sum_{i=1}^{N_{g}} p(i, j)$,

$p_{g}$ the sum distribution of the number of zones with grey level $i$, where $p_{g}(i)=\sum_{j=1}^{N_{z}} p(i, j)$,

$N_{p}$ the number of voxels in the image, where $N_{p}=\sum_{j=1}^{N_{d}} j p_{d}$,

$p_{n}(i, j)$ the normalized size-zone matrix, where $p_{n}(i, j)=\frac{p(i, j)}{N_{s}}$,

$\mu_{d}$ the mean distance, where $\mu_{d}=\sum_{i=1}^{N_{g}} \sum_{j=1}^{N_{d}} j p_{n}(i, j \mid \theta)$,

$\mu_{g}$ the mean grey level, where $\mu_{g}=\sum_{i=1}^{N_{g}} \sum_{j=1}^{N_{d}} i p_{n}(i, j \mid \theta)$.

124. Small distance Emphasis (SDE)

$$
S D E=\frac{1}{N_{s}} \sum_{j=1}^{N_{d}} \frac{p_{d}}{j^{2}}
$$

125. Large distance Emphasis (LDE)

$$
L D E=\frac{1}{N_{s}} \sum_{j=1}^{N_{d}} j^{2} p_{d}
$$


126. Intensity Non-Uniformity (IN)

$$
I N=\frac{1}{N_{s}} \sum_{i=1}^{N_{g}} p_{g}^{2}
$$

127. Intensity Non-Uniformity Normalized (INN)

$$
I N N=\frac{1}{N_{S}^{2}} \sum_{i=1}^{N_{g}} p_{g}^{2}
$$

128. Distance-zone Non-Uniformity (DZN)

$$
D Z N=\frac{1}{N_{s}} \sum_{j=1}^{N_{d}} p_{d}^{2}
$$

129. Distance-zone Non-Uniformity Normalized (DZNN)

$$
D Z N N=\frac{1}{N_{s}^{2}} \sum_{j=1}^{N_{d}} p_{d}^{2}
$$

130. Zone Percentage (ZP)

$$
Z P=\frac{N_{s}}{N_{p}}
$$

131. Low intensity Emphasis (LIE)

$$
L I E=\frac{1}{N_{s}} \sum_{i=1}^{N_{g}} \frac{p_{g}}{i^{2}}
$$

132. High intensity Emphasis (HIE)

$$
H I E=\frac{1}{N_{s}} \sum_{i=1}^{N_{g}} i^{2} p_{g}
$$


133. Low intensity small distance Emphasis (LISDE)

$$
\text { LISDE }=\frac{1}{N_{s}} \sum_{i=1}^{N_{g}} \sum_{j=1}^{N_{d}} \frac{p(i, j)}{i^{2} j^{2}}
$$

134. High intensity small distance Emphasis (HISDE)

$$
H I S D E=\frac{1}{N_{s}} \sum_{i=1}^{N_{g}} \sum_{j=1}^{N_{d}} \frac{p(i, j) i^{2}}{j^{2}}
$$

135. Low intensity large distance Emphasis (LILDE)

$$
L I L D E=\frac{1}{N_{s}} \sum_{i=1}^{N_{g}} \sum_{j=1}^{N_{d}} \frac{p(i, j) j^{2}}{i^{2}}
$$

136. High intensity large distance Emphasis (HILDE)

$$
H I L D E=\frac{1}{N_{s}} \sum_{i=1}^{N_{g}} \sum_{j=1}^{N_{d}} p(i, j) i^{2} j^{2}
$$

137. Intensity variance (IV)

$$
I V=\sum_{i=1}^{N_{g}} \sum_{j=1}^{N_{d}}\left(i-\mu_{g}\right)^{2} p_{n}(i, j)
$$

138. Distance-zone variance (DZV)

$$
S Z V=\sum_{i=1}^{N_{g}} \sum_{j=1}^{N_{d}}\left(j-\mu_{d}\right)^{2} p_{n}(i, j)
$$

139. Distance-zone entropy (DZE)

$$
D Z E=\sum_{i=1}^{N_{g}} \sum_{j=1}^{N_{d}} p_{n}(i, j) \log _{2}\left[p_{n}(i, j)\right]
$$




\section{Neighborhood Grey tone difference matrix based features}

Neighborhood Grey tone difference matrix (NGTDM) based features, as described by Amadasun and King (14). The $i$ th entry of the NGTDM $s(i \mid d)$ is the sum of grey level differences of voxels with intensity $i$ and the average intensity $A_{i}$ of their neighboring voxels within a distance $d$. In contrast to the original paper, a complete neighborhood is not required and $A_{i}$ is determined over the valid voxels. Let: $n_{i}$ be the number of voxels with grey level $i$,

$N_{v}=\sum n_{i}$, the total number of voxels (defined as $n^{2}$ by Amadasun and King (14)),

$s(i)=\left\{\begin{array}{cc}\sum_{n_{i}}\left|i-A_{i}\right| & \text { for } n_{i}>0 \\ 0 & \text { otherwise }\end{array}\right.$, generalized for any distance $d$,

$N_{g}$ be the maximum discrete intensity level in the image,

$p(i)=\frac{n_{i}}{N_{v}}$, the probability of grey level $i$,

$N_{p}$, the total number of grey levels present in the image.

\section{Coarseness}

$$
\text { coarseness }=\frac{1}{\varepsilon+\sum_{i=i}^{N_{g}} p(i) s(i)}
$$

Where $\varepsilon$ is a small number to prevent coarseness becoming infinite.

\section{Contrast}

$$
\text { contrast }=\left(\frac{1}{N_{p}\left(1-N_{p}\right)} \sum_{i=1}^{N_{g}} \sum_{j=1}^{N_{g}} p(i) p(j)(i-j)^{2}\right)\left(\frac{1}{N_{v}} \sum_{i=i}^{N_{g}} s(i)\right)
$$

142. Busyness

$$
\text { busyness }=\frac{\sum_{i=i}^{N_{g}} p(i) s(i)}{\sum_{i=i}^{N_{g}} \sum_{j=i}^{N_{g}}|i p(i)-j p(j)|}, \quad p(i) \neq 0, \quad p(j) \neq 0
$$

143. Complexity

$$
\text { complexity }=\frac{1}{N_{v}} \sum_{i=i}^{N_{g}} \sum_{j=i}^{N_{g}}|i-j| \frac{p(i) s(i)+p(j) s(j)}{p(i)+p(j)}, \quad p(i) \neq 0, p(j) \neq 0
$$


144. Strength

$$
\text { strength }=\frac{\sum_{i=i}^{N_{g}} \sum_{j=i}^{N_{g}}[p(i)+p(j)](i-j)^{2}}{\varepsilon+\sum_{i=i}^{N_{g}} s(i)}, \quad p(i) \neq 0, p(j) \neq 0
$$




\section{Neighboring Grey level dependence matrix based features}

Neighboring Grey level dependence matrix (NGLDM) based features, as described by Sun and Wee (15). $\operatorname{NGLDM}$ features are invariant under spatial rotation. The $(i, j)$ th entry of the $\operatorname{NGLDM} p(i, j \mid d, a)$ describes the number of neighborhoods with center voxel grey level (i.e. intensity value) $i$ and dependence (i.e. number of dependent voxels) $k=j-1$. A neighborhood are all voxels within a distance $d$ from the center voxel. The center voxel and a neighboring voxel are dependent if their absolute grey value difference $\leq a$, the dependency coarseness parameter. The features originally specified by Sun and Wee are analogous to the GLRLM and GLSZM features, and the feature set is extended accordingly. Let:

$p(i, j)$ be the $(i, j)$ th entry in the given $\operatorname{NGLDM} p$, generalized for any $d$ and $a$,

$N_{g}$ the number of discrete intensity values in the image,

$N_{d}$ the maximum dependence value,

$N_{s}$ the total number of neighborhoods, where $N_{s}=\sum_{i=1}^{N_{g}} \sum_{j=1}^{N_{z}} p(i, j)$,

$p_{d}$ the sum distribution of the number of neighborhoods with dependence $j=k+1$, where $p_{d}(j)=$ $\sum_{i=1}^{N_{g}} p(i, j)$,

$p_{g}$ the sum distribution of the number of neighborhoods with center voxel grey level $i$, where $p_{g}(i)=$ $\sum_{j=1}^{N_{d}} p(i, j)$,

$p_{n}(i, j)$ the normalized NGLDM, where $p_{n}(i, j)=\frac{p(i, j)}{N_{s}}$,

$\mu_{d}$ the mean dependence, where $\mu_{r}=\sum_{i=1}^{N_{g}} \sum_{j=1}^{N_{z}} j p_{n}(i, j \mid \theta)$,

$\mu_{g}$ the mean grey level, where $\mu_{g}=\sum_{i=1}^{N_{g}} \sum_{j=1}^{N_{z}} i p_{n}(i, j \mid \theta)$.

Note: By definition, the number of voxels in the image $\left(N_{p}\right)$ equals the total number of neighborhoods $\left(N_{S}\right)$, since in our implementation every voxel is considered to have a neighborhood. Feature "dependence percentage" $\left(\frac{N_{S}}{N_{p}}\right)$, which is the equivalent to run-length feature "run percentage" (RP; 98$)$, is therefore omitted, because it will always evaluate to 1 .

145. Small Dependence Emphasis (SDE)

$$
S D E=\frac{1}{N_{s}} \sum_{j=1}^{N_{d}} \frac{p_{d}}{j^{2}}
$$

This feature is also called Small Number Emphasis (15). 
146. Large Dependence Emphasis (LDE)

$$
L D E=\frac{1}{N_{s}} \sum_{j=1}^{N_{d}} j^{2} p_{d}
$$

This feature is also called Large Number Emphasis (15).

147. Grey-level Non-Uniformity (GLN)

$$
G L N=\frac{1}{N_{s}} \sum_{i=1}^{N_{g}} p_{g}^{2}
$$

148. Grey-level Non-Uniformity Normalized (GLNN)

$$
G L N N=\frac{1}{N_{S}^{2}} \sum_{i=1}^{N_{g}} p_{g}^{2}
$$

149. Dependence Non-Uniformity (DN)

$$
D N=\frac{1}{N_{s}} \sum_{j=1}^{N_{d}} p_{z}^{2}
$$

This feature is also called Number Nonuniformity (15).

150. Dependence Non-Uniformity Normalized (DNN)

$$
D N N=\frac{1}{N_{S}^{2}} \sum_{j=1}^{N_{z}} p_{z}^{2}
$$

151. Low Grey-level Emphasis (LGE)

$$
L G E=\frac{1}{N_{s}} \sum_{i=1}^{N_{g}} \frac{p_{g}}{i^{2}}
$$

152. High Grey-level Emphasis (HGE)

$$
H G E=\frac{1}{N_{S}} \sum_{i=1}^{N_{g}} i^{2} p_{g}
$$


153. Low Grey-level small Dependence Emphasis (LGSDE)

$$
L G S D E=\frac{1}{N_{s}} \sum_{i=1}^{N_{g}} \sum_{j=1}^{N_{d}} \frac{p(i, j)}{i^{2} j^{2}}
$$

154. High Grey-level small Dependence Emphasis (HGSDE)

$$
H G S D E=\frac{1}{N_{s}} \sum_{i=1}^{N_{g}} \sum_{j=1}^{N_{d}} \frac{p(i, j) i^{2}}{j^{2}}
$$

155. Low Grey-level large Dependence Emphasis (LGLDE)

$$
L G L D E=\frac{1}{N_{s}} \sum_{i=1}^{N_{g}} \sum_{j=1}^{N_{d}} \frac{p(i, j) j^{2}}{i^{2}}
$$

156. High Grey-level large Dependence Emphasis (HGLDE)

$$
H G L D E=\frac{1}{N_{s}} \sum_{i=1}^{N_{g}} \sum_{j=1}^{N_{d}} p(i, j) i^{2} j^{2}
$$

157. Grey-level variance (GLV)

$$
G L V=\sum_{i=1}^{N_{g}} \sum_{j=1}^{N_{d}}\left(i-\mu_{g}\right)^{2} p_{n}(i, j)
$$

158. Dependence variance (DV)

$$
D V=\sum_{i=1}^{N_{g}} \sum_{j=1}^{N_{d}}\left(j-\mu_{d}\right)^{2} p_{n}(i, j)
$$


159. Dependence entropy (DE), also called Entropy (15)

$$
D E=\sum_{i=1}^{N_{g}} \sum_{j=1}^{N_{d}} p_{n}(i, j) \log _{2}\left[p_{n}(i, j)\right]
$$

Note: the definition of entropy by Sun and Wee (15) uses the dependence counts $(p)$ instead of the dependence propabilities $\left(p_{n}\right)$.

160. Second moment (SM) (15)

$$
S M=\frac{\sum_{i=1}^{N_{g}} \sum_{j=1}^{N_{d}} p(i, j)^{2}}{N_{s}}
$$

Note: for this feature, defined by Sun and Wee (15), there is no grey-level run-length equivalent 


\section{Wavelet features}

Wavelet transform effectively decouples textural information by decomposing the original image, in a similar manner as Fourier analysis, in low- and high-frequencies. In this study a discrete, one-level and undecimated three dimensional wavelet transform was applied to each CT and CBCT image, which decomposes the original image $X$ into 8 decompositions. Consider $L$ and $H$ to be a low-pass (i.e. a scaling) and, respectively, a high-pass (i.e. a wavelet) function, and the wavelet decompositions of $X$ to be labeled as $X_{L L L}, X_{L L H}, X_{L H L}, X_{L H H}, X_{H L L}, X_{H L H}, X_{H H L}$ and $X_{H H H}$. For example, $X_{L L H}$ is then interpreted as the high-pass sub band, resulting from directional filtering of $X$ with a low-pass filter along $\mathrm{x}$-direction, a low pass filter along $\mathrm{y}$-direction and a high-pass filter along $\mathrm{z}$-direction and is constructed as:

$$
X_{L L H}(i, j, k)=\sum_{p=1}^{N_{L}} \sum_{q=1}^{N_{L}} \sum_{r=1}^{N_{H}} L(p) L(q) H(r) X(i+p, j+q, k+r)
$$

Where $N_{L}$ is the length of filter $L$ and $N_{H}$ is the length of filter $H$. The other decompositions are constructed in a similar manner, applying their respective ordering of low or high-pass filtering in $\mathrm{x}, \mathrm{y}$ and z-direction. Wavelet decomposition of the image $X$ is schematically depicted in Figure 7. Since the applied wavelet decomposition is undecimated, the size of each decomposition is equal to the original image and each decomposition is shift invariant. Because of these properties, the original tumor delineation of the gross tumor volume (GTV) can be applied directly to the decompositions after wavelet transform. In this study "Coiflet 1" wavelet was applied. For each decomposition we computed the first order gray level statistics and the textural features (GLCM, GLRLM, GLSZM). 


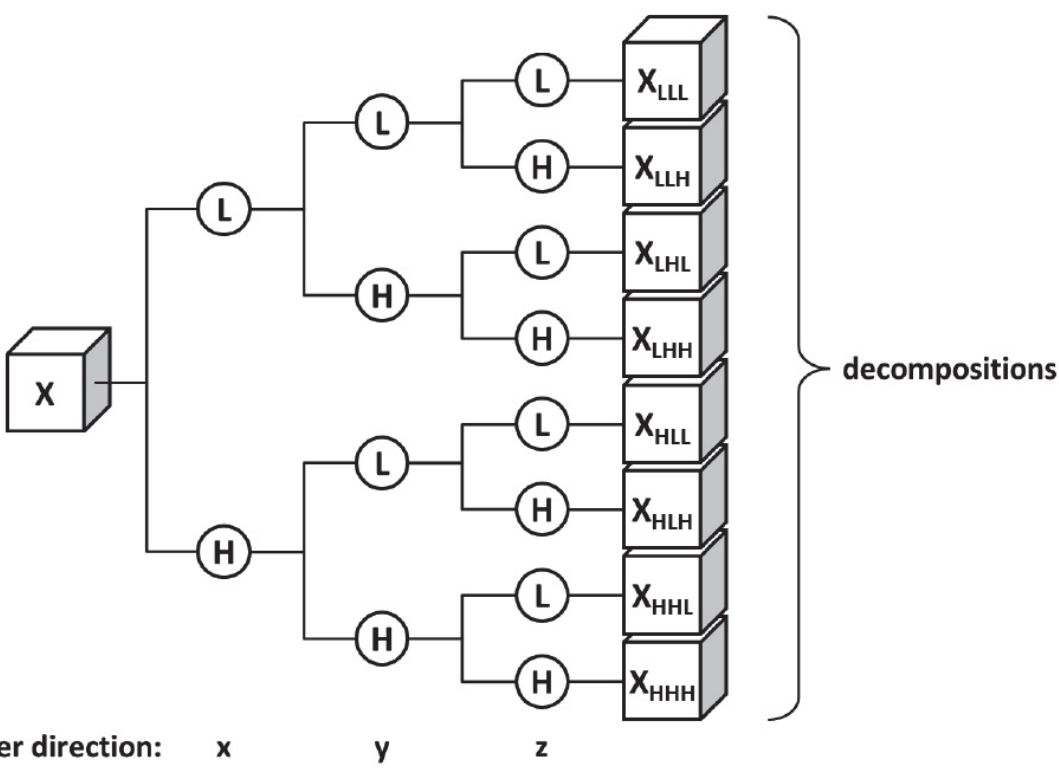

Figure 8: Schematic of the undecimated three dimensional wavelet transform applied to each CT/CBCT image. The original image $X$ is decomposed into 8 decompositions, by directional low-pass (i.e. a scaling) and high-pass (i.e. a wavelet) filtering: $X_{L L L}, X_{L L H}, X_{L H L}, X_{L H H}, X_{H L L}, X_{H L H}, X_{H H L}$ and $X_{H H H}$. 


\section{Laplacian of Gaussian features}

The Laplacian of an image brings out areas of rapid intensity change and is usually used for edge detection. A Gaussian filter is applied prior to the Laplacian to smooth the image and reduce noise. Textural properties representing features of different degrees of coarseness can then be calculated. The equation of a LoG with a 2D kernel:

$$
\operatorname{LoG}(x, y)=-\frac{1}{\pi \sigma^{4}}\left[1-\frac{x^{2}+y^{2}}{2 \sigma^{2}}\right] e^{-\frac{x^{2}+y^{2}}{2 \sigma^{2}}}
$$

Texture size (fine to coarse) is highlighted by modifying the Gaussian radius parameter $\sigma$ (e.g., from 0.5 $\mathrm{mm}$ to $5 \mathrm{~mm}$, with $0.5 \mathrm{~mm}$ increments). Each value of $\sigma$ provides a filtered image. First-order gray-level statistics (described earlier) are determined for each filtered image, as well as for only the positive part of each filtered image.

Figure 8.5 Feature descriptions 


\section{Supplementary data references}

1. Al-Kadi OS, ast, Watson D. Texture Analysis of Aggressive and Nonaggressive Lung Tumor CE CT Images. IEEE Transactions on Biomedical Engineering. 2008;55(7):1822-30.

2. Wahl RL, Jacene H, Kasamon Y, Lodge MA. From RECIST to PERCIST: Evolving Considerations for PET response criteria in solid tumors. Journal of nuclear medicine : official publication, Society of Nuclear Medicine. 2009;50 Suppl 1:122s-50s.

3. Aerts HJ, Velazquez ER, Leijenaar RT, Parmar C, Grossmann P, Carvalho S, et al. Decoding tumour phenotype by noninvasive imaging using a quantitative radiomics approach. Nat Commun. 2014;5:4006.

4. Sousa JR, Silva AC, de Paiva AC, Nunes RA. Methodology for automatic detection of lung nodules in computerized tomography images. Comput Methods Programs Biomed. 2010;98(1):1-14.

5. Haralick RM, Shanmugam K, Dinstein I. Textural Features of Image Classification. IEEE T Syst Man Cyb. 1973;SMC-3(6):610-21.

6. Clausi DA. An analysis of co-occurrence texture statistics as a function of grey level quantization. Canadian Journal of remote sensing. 2002;28(1):45-62.

7. Tsatsoulis LSaC. Texture Analysis of SAR Sea Ice Imagery Using Gray Level Co-Occurrence Matrices. IEEE Transactions on Geoscience and Remote Sensing. 1999;37(2).

8. Galloway M. Texture analysis using gray level run lengths. Comput Vision Graph. 1975;4:172-9.

9. Albregtsen F, Nielsen B, Danielsen HE, editors. Adaptive gray level run length features from class distance matrices. Pattern Recognition, 2000 Proceedings 15th International Conference on; 20002000.

10. Thibault GF, B; Navarro, C; Pereira, S. Texture indexes and gray level size zone matrix: application to cell nuclei classification. Pattern Recognition Inf Process. 2009:140-5.

11. Tixier F, Hatt M, Le Rest CC, Le Pogam A, Corcos L, Visvikis D. Reproducibility of tumor uptake heterogeneity characterization through textural feature analysis in 18F-FDG PET. J Nucl Med. 2012;53(5):693-700.

12. Tixier F, Le Rest CC, Hatt M, Albarghach N, Pradier O, Metges JP, et al. Intratumor heterogeneity characterized by textural features on baseline 18F-FDG PET images predicts response to concomitant radiochemotherapy in esophageal cancer. J Nucl Med. 2011;52(3):369-78.

13. Thibault G, Angulo J, Meyer F. Advanced Statistical Matrices for Texture Characterization: Application to Cell Classification. IEEE Transactions on Biomedical Engineering. 2014;61(3):630-7.

14. Amadasun M, King R. Textural features corresponding to textural properties. Systems, Man and Cybernetics, IEEE Transactions on. 1989;19(5):1264-74.

15. Sun C, Wee WG. Neighboring gray level dependence matrix for texture classification. Computer Vision, Graphics, and Image Processing. 1983;23(3):341-52. 


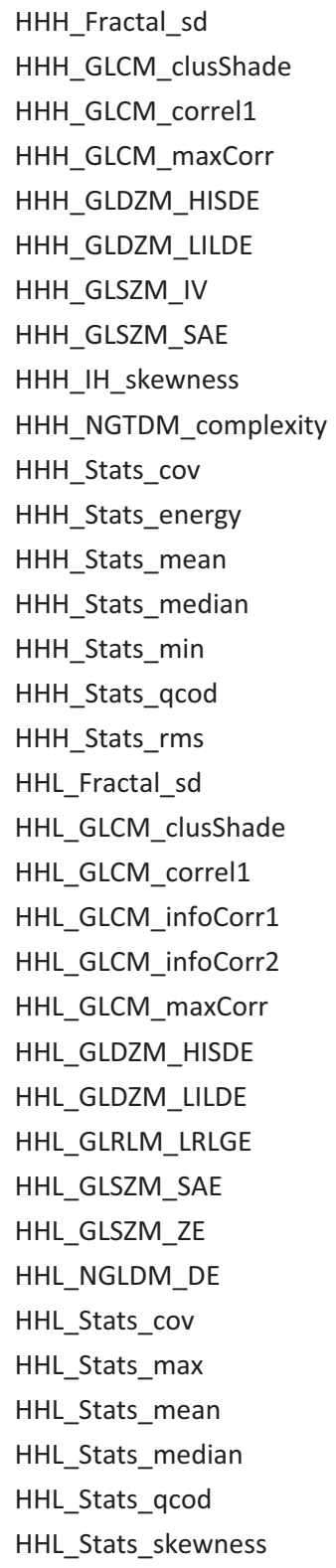


HLH_Fractal_sd

HLH_GLCM_clusShade

HLH_GLCM_correl1

HLH_GLCM_infoCorr1

HLH_GLCM_maxCorr

HLH_GLDZM_HISDE

HLH_GLDZM_LILDE

HLH_GLSZM_IV

HLH_GLSZM_SAE

HLH_GLSZM_ZE

HLH_NGTDM_complexity

HLH_Stats_cov

HLH_Stats_max

HLH_Stats_mean

HLH_Stats_median

HLH_Stats_qcod

HLH_Stats_skewness

HLL_Fractal_sd

HLL_GLCM_clusShade

HLL_GLCM_correl1

HLL_GLCM_infoCorr1

HLL_GLCM_infoCorr2

HLL_GLCM_maxCorr

HLL_GLDZM_LILDE

HLL_GLDZM_SDE

HLL_GLRLM_LRLGE

HLL_GLRLM_RE

HLL_GLSZM_IV

HLL_GLSZM_SAE

HLL_GLSZM_ZE

HLL_IH_mingradi

HLL_IH_mode

HLL_IH_skewness

HLL_NGLDM_DE

HLL_NGLDM_LGLDE

HLL_Stats_cov

HLL_Stats_mean

HLL_Stats_min

HLL_Stats_p10

HLL_Stats_qcod

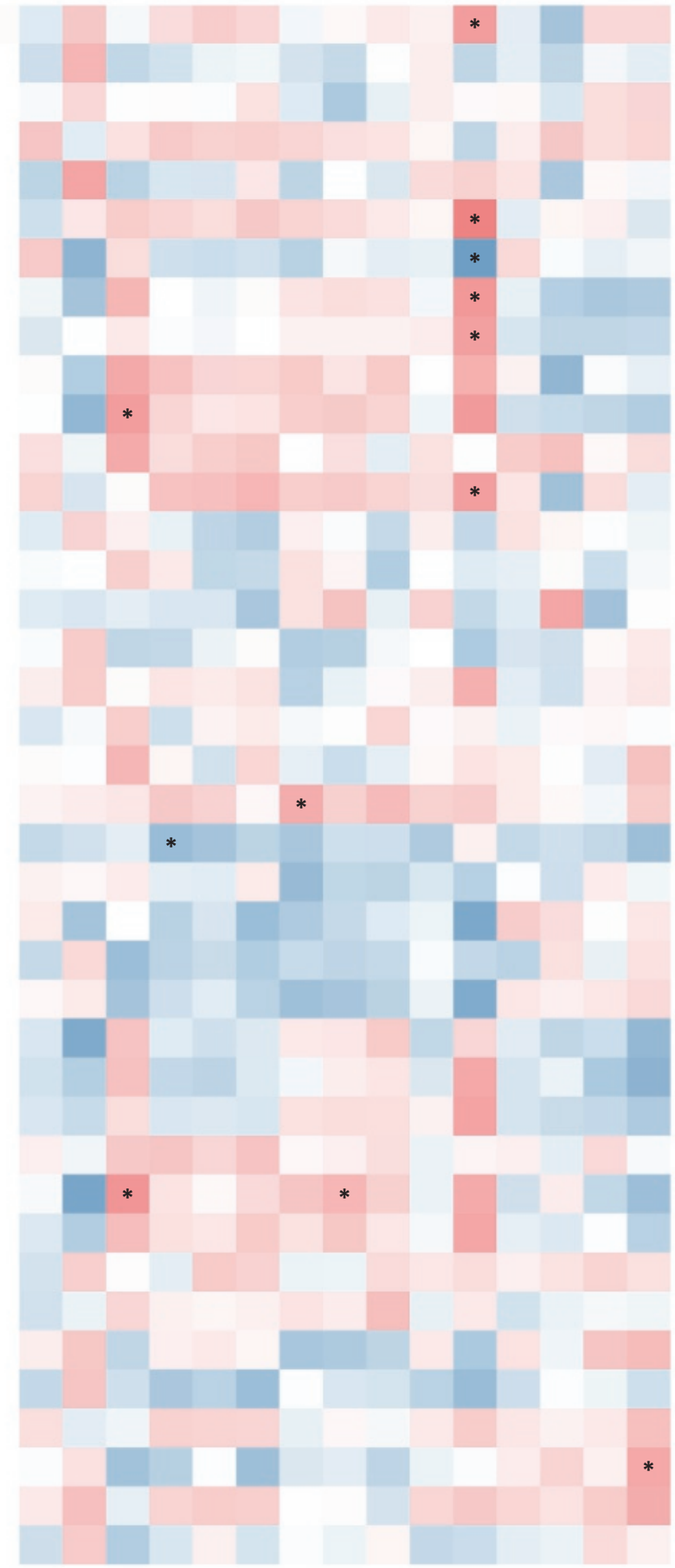


HLL_Stats_range

LHH_Fractal_average

LHH_Fractal_sd

LHH_GLCM_clusShade

LHH_GLCM_correl1

LHH_GLCM_infoCorr1

LHH_GLCM_maxCorr

LHH_GLDZM_DZE

LHH_GLDZM_HISDE

LHH_GLDZM_LILDE

LHH_GLSZM_IV

LHH_GLSZM_SAE

LHH_GLSZM_ZE

LHH_NGLDM_DV

LHH_Stats_cov

LHH_Stats_mean

LHH_Stats_median

LHH_Stats_qcod

LHH_Stats_skewness

LHL_Fractal_sd

LHL_GLCM_clusShade

LHL_GLCM_correl1

LHL_GLCM_infoCorr1

LHL_GLCM_maxCorr

LHL_GLDZM_DZE

LHL_GLDZM_HISDE

LHL_GLDZM_LILDE

LHL_GLRLM_LRLGE

LHL_GLSZM_IV

LHL_GLSZM_SAE

LHL_GLSZM_ZE

LHL_NGLDM_DV

LHL_NGLDM_LGLDE

LHL_Stats_mean

LHL_Stats_min

LHL_Stats_qcod

LHL_Stats_skewness

LLH_Fractal_average

LLH_Fractal_sd

LLH_GLCM_clusShade

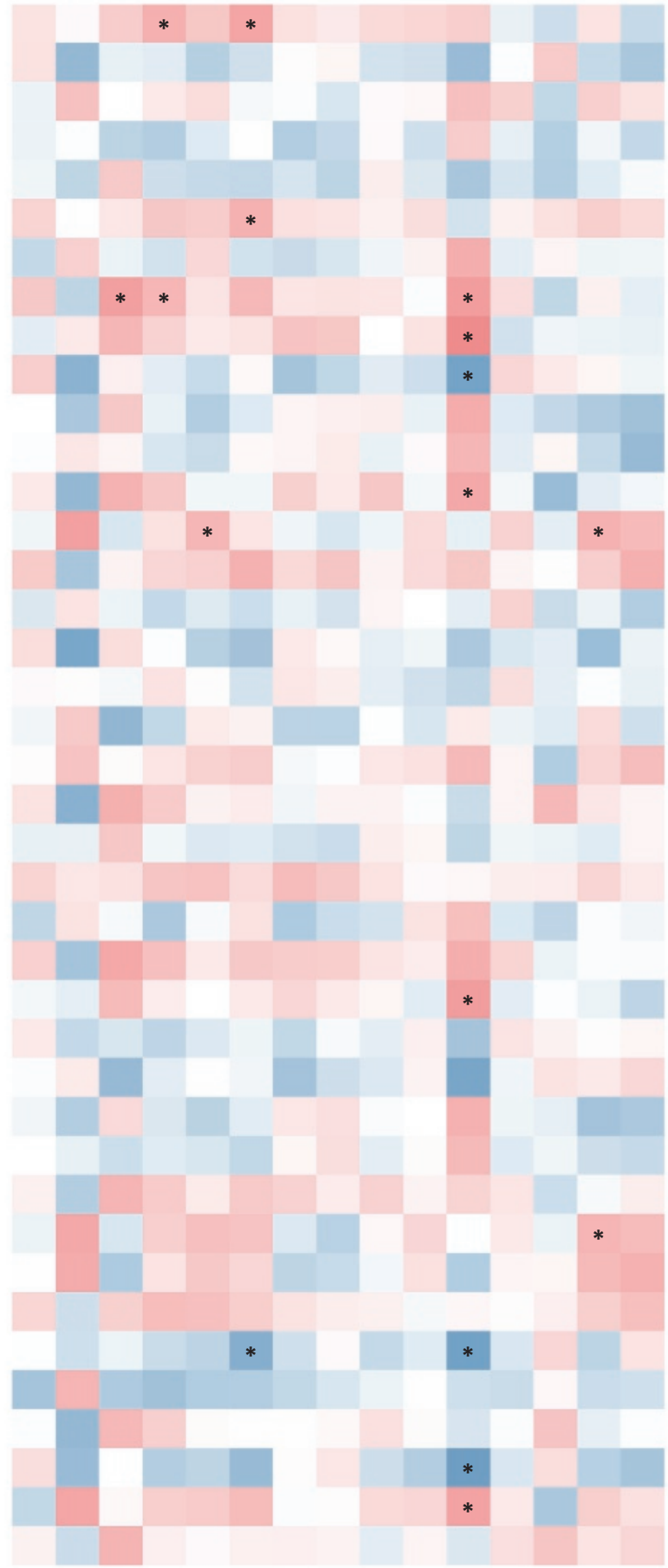



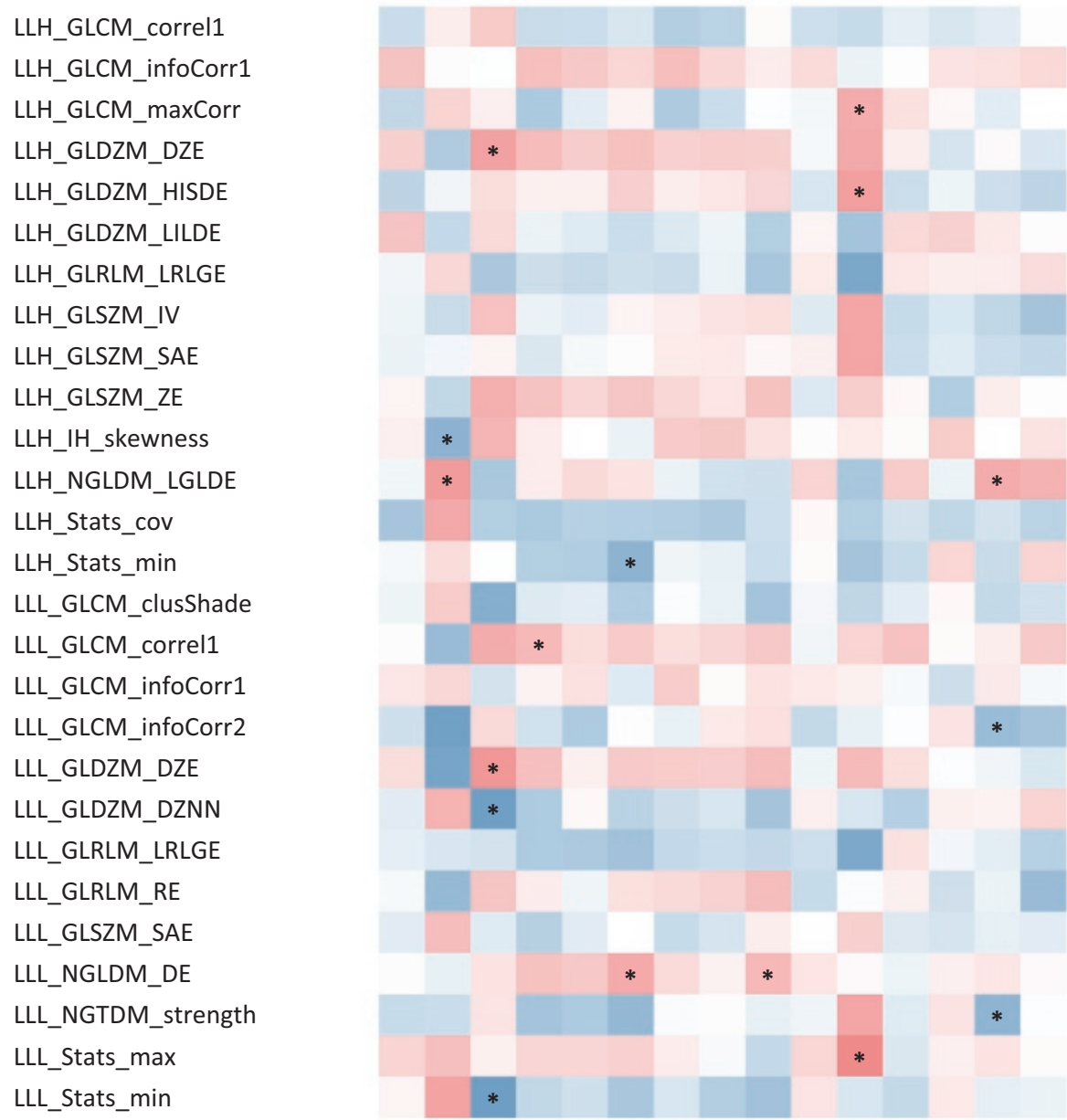

Figure 8.6 Associations between semantic and filtered radiomic features for the tumor. 


\section{Feature}

HHH_Fractal_average

$\mathrm{HHH}$ _Fractal_sd

HHH_GLCM_clusShade

HHH_GLCM_maxCorr

HHH_GLDZM_DZN

HHH_GLDZM_HISDE

HHH_GLDZM_LILDE

HHH_GLSZM_IV

HHH_GLSZM_SAE

$\mathrm{HHH}$ _lH_iqr

HHH_IH_mediand

HHH_lH_qcod

HHH_IH_skewness

HHH_NGLDM_LGSDE

$\mathrm{HHH}$ _Stats_cov

HHH_Stats_max

HHH_Stats_mean

HHH_Stats_median

HHH_Stats_qcod

HHL_Fractal_sd

HHL_GLCM_clusShade

HHL_GLCM_correl1

HHL_GLCM_infoCorr1

HHL_GLCM_infoCorr2

HHL_GLCM_maxCorr

HHL_GLDZM_LILDE

HHL_GLSZM_LISAE

HHL_GLSZM_SZNN

HHL_GLSZM_ZE

HHL_IH_iqr

HHL_IH_mediand

HHL_IH_qcod

HHL_NGLDM_LGSDE

HHL_Stats_cov

HHL_Stats_mean

HHL_Stats_median
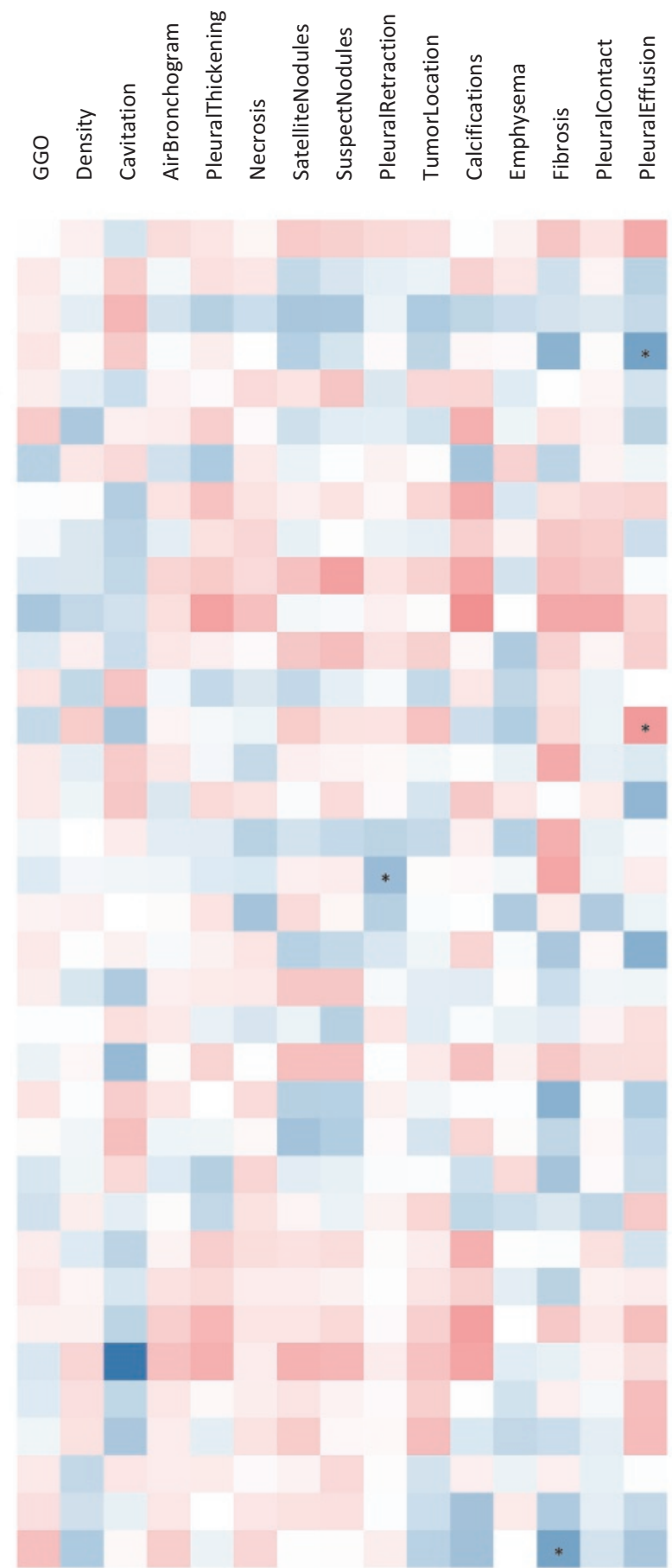
HHL_Stats_min

HHL_Stats_qcod

HHL_Stats_skewness

HLH_GLCM_clusShade

HLH_GLCM_correl1

HLH_GLCM_infoCorr1

HLH_GLCM_maxCorr

HLH_GLDZM_HISDE

HLH_GLDZM_LILDE

HLH_GLSZM_SZNN

HLH_GLSZM_ZE

HLH_IH_iqr

HLH_IH_mediand

HLH_IH_qcod

HLH_NGLDM_LGSDE

HLH_Stats_cov

HLH_Stats_mean

HLH_Stats_median

HLH_Stats_min

HLH_Stats_qcod

HLH_Stats_skewness

HLL_GLCM_correl1

HLL_GLCM_infoCorr1

HLL_GLCM_infoCorr2

HLL_GLDZM_DZN

HLL_GLDZM_HISDE

HLL_GLDZM_LILDE

HLL_GLSZM_LISAE

HLL_GLSZM_SAE

HLL_GLSZM_ZE

HLL_IH_cov

HLL_IH_iqr

HLL_IH_kurtosis

HLL_IH_mediand

HLL_IH_qcod

HLL_NGLDM_DE

HLL_NGLDM_DV

HLL_NGLDM_LGSDE

HLL_Stats_cov

HLL_Stats_max

HLL_Stats_mean

HLL_Stats_median

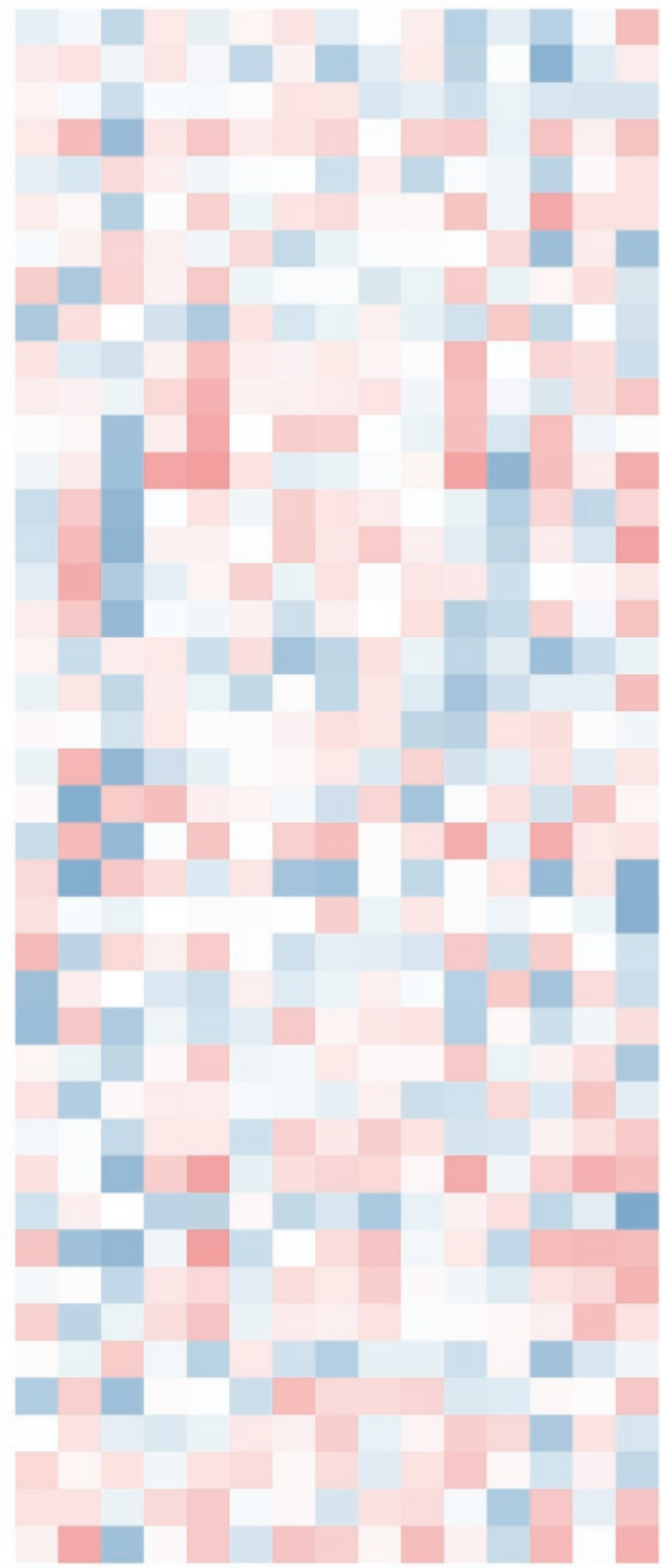


HLL_Stats_min

HLL_Stats_qcod

HLL_Stats_skewness

LHH_Fractal_average

LHH_Fractal_sd

LHH_GLCM_correl1

LHH_GLCM_infoCorr1

LHH_GLCM_infoCorr2

LHH_GLCM_maxCorr

LHH_GLDZM_DZN

LHH_GLDZM_HISDE

LHH_GLDZM_LILDE

LHH_GLRLM_RE

LHH_GLSZM_LISAE

LHH_GLSZM_SZNN

LHH_GLSZM_ZE

LHH_IH_energy

LHH_IH_iqr

LHH_IH_mediand

LHH_IH_qcod

LHH_NGLDM_LGSDE

LHH_NGTDM_strength

LHH_Stats_cov

LHH_Stats_max

LHH_Stats_mean

LHH_Stats_median

LHH_Stats_qcod

LHH_Stats_skewness

LHH_Stats_var

LHL_GLCM_clusShade

LHL_GLCM_correl1

LHL_GLCM_inverseVar

LHL_GLCM_maxCorr

LHL_GLDZM_DZN

LHL_GLDZM_HISDE

LHL_GLDZM_LILDE

LHL_GLRLM_RE

LHL_GLSZM_SZNN

LHL_IH_cov

LHL_IH_iqr

LHL_IH_mediand

LHL_IH_qcod

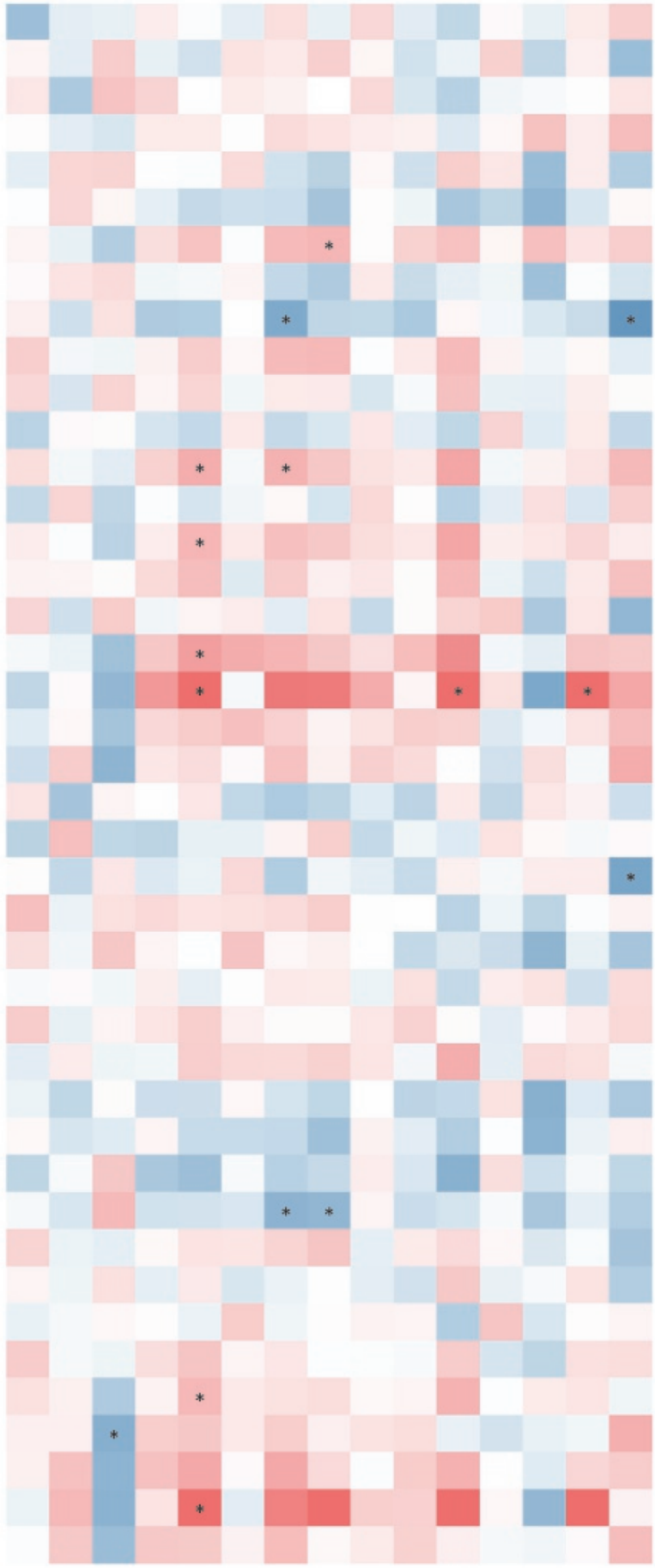


LHL_IH_skewness

LHL_NGLDM_DV

LHL_Stats_cov

LHL_Stats_max

LHL_Stats_mean

LHL_Stats_min

LHL_Stats_qcod

LHL_Stats_rms

LLH_GLCM_clusShade

LLH_GLCM_maxCorr

LLH_GLDZM_DZN

LLH_GLDZM_HISDE

LLH_GLDZM_LILDE

LLH_GLRLM_RE

LLH_GLSZM_SZNN

LLH_IH_cov

LLH_IH_iqr

LLH_IH_mediand

LLH_IH_qcod

LLH_NGLDM_DV

LLH_Stats_cov

LLH_Stats_max

LLH_Stats_mean

LLH_Stats_median

LLH_Stats_min

LLH_Stats_qcod

LLH_Stats_skewness

LLL_GLCM_infoCorr1

LLL_GLCM_invDiffmomnor

LLL_GLCM_inverseVar

LLL_GLDZM_HISDE

LLL_GLDZM_LILDE

LLL_GLDZM_LISDE

LLL_GLRLM_LRHGE

LLL_IH_cov

LLL_IH_kurtosis

LLL_IH_maxgradi

LLL_IH_mediand

LLL_IH_qcod

LLL_NGLDM_HGLDE

LLL_NGLDM_LGSDE

LLL_Stats_cov

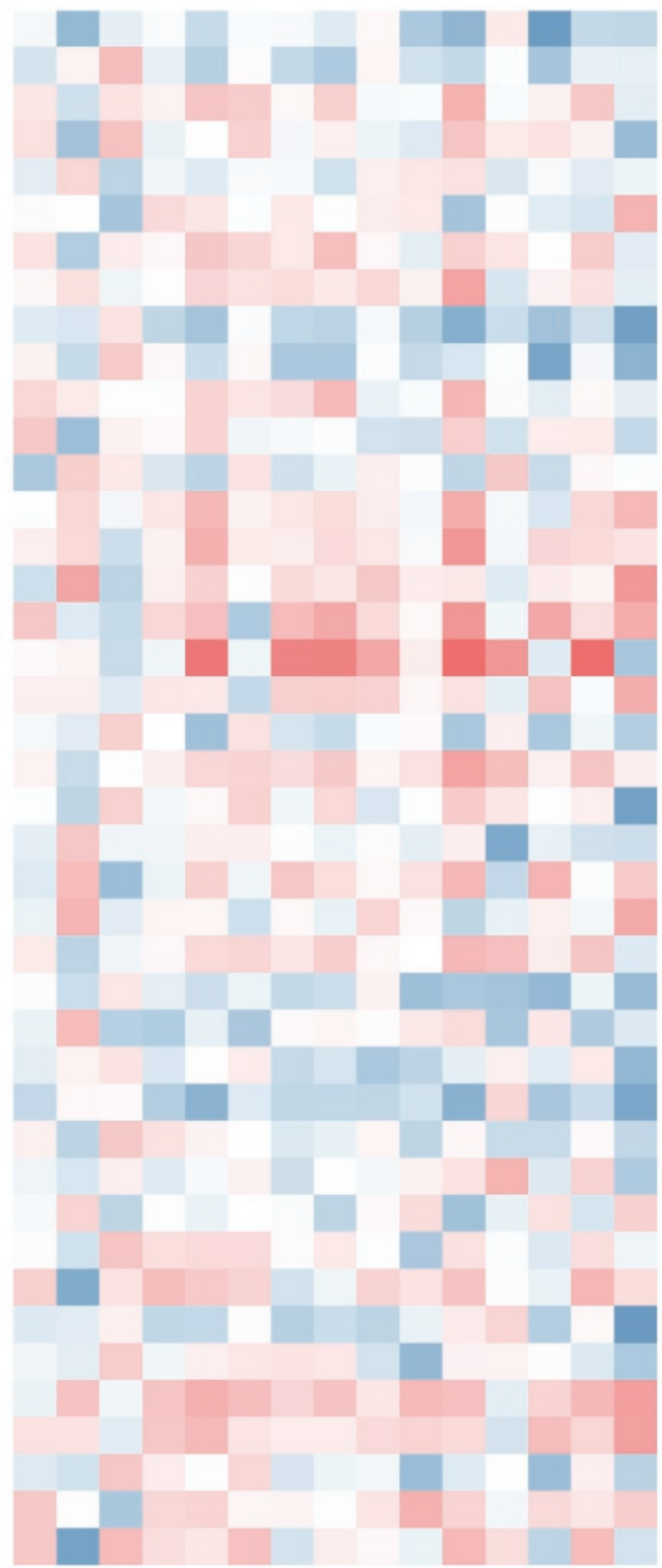




\section{LLL_Stats_energy \\ LLL_Stats_min \\ LLL_Stats_qcod}

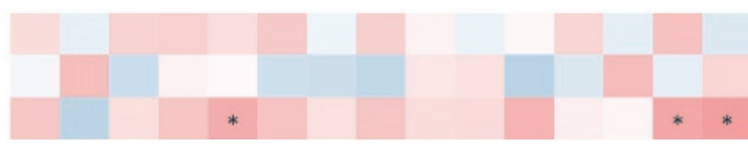

Figure 8.7 Associations between semantic and filtered radiomic features for the ipsilateral lung. 


\begin{tabular}{|c|c|c|c|}
\hline & Tumor & Lung & Combined \\
\hline $\begin{array}{l}\text { Air } \\
\text { bronchogram }\end{array}$ & $\begin{array}{l}\text { Wavelet_LHH_GLDZM_DZE_gtv } \\
\text { Wavelet_HHH_Stats_energy_gtv } \\
\text { Wavelet_HLH_Stats_median_gtv } \\
\text { Wavelet_HHL_GLSZ_SAE_gtv } \\
\text { Wavelet_HLH_Stats_qcod_gtv } \\
\text { Wavelet_LLL_NGLDM_DE_gtv } \\
\text { GLSZM_ZE_gtv } \\
\text { Wavelet_LHL_GLSZM_SAE_gtv } \\
\text { Wavelet_LHH_Stats_qcod_gtv } \\
\text { Wavelet_LLL_GLRLM_LRLGE_gtv }\end{array}$ & & $\begin{array}{l}\text { GLDZM_HISDE_lung } \\
\text { Wavelet_HLL_GLCM_correl1_lung } \\
\text { Shape_volumeDensityBE_lung } \\
\text { Wavelet_HLL_GLCM_infoCorr2_gtv } \\
\text { Wavelet_LLH_GLDZM_HISDE_lung } \\
\text { Wavelet_LLH_Stats_min_gtv } \\
\text { Wavelet_HHL_Stats_qcod_gtv } \\
\text { Shape_areaDensityBE_gtv } \\
\text { Wavelet_HHH_Stats_cov_gtv } \\
\text { Wavelet_LHL_NGLDM_DV_lung }\end{array}$ \\
\hline Necrosis & $\begin{array}{l}\text { Wavelet_HLL_GLDZM_SDE_gtv } \\
\text { Wavelet_LLH_GLDZM_LILDE_gtv } \\
\text { Wavelet_LLL_NGTDM_strength_gtv } \\
\text { Wavelet_HHH_GLDZM_LILDE_gtv } \\
\text { Wavelet_LHL_GLDZM_DZE_gtv } \\
\text { Wavelet_LLL_GLDM_DZNN_gtv } \\
\text { Wavelet_HHL_Stats_qcod_gtv } \\
\text { Wavelet_LHH_Stats_qcod_gtv } \\
\text { Wavelet_LLH_GLDZM_DZE_gtv } \\
\text { Stats_median_gtv }\end{array}$ & & $\begin{array}{l}\text { Wavelet_HHH_Stats_min_gtv } \\
\text { Wavelet_LLL_NGTDM_strength_gtv } \\
\text { Wavelet_HHH_GLDM_LILDE_gtv } \\
\text { Wavelet_HHL_GLCM_infoCorr1_gtv } \\
\text { Wavelet_LHH_GLCM_infoCorr1_gtv } \\
\text { Wavelet_HHH_Stats_median_gtv } \\
\text { Wavelet_HLL_Stats_median_lung } \\
\text { Shape_volumeDensityBE_lung } \\
\text { Wavelet_HHH_GLCM_maxCorr_lung } \\
\text { Wavelet_LHL_Stats_min_gtv }\end{array}$ \\
\hline $\begin{array}{l}\text { Pleural } \\
\text { effusion }\end{array}$ & $\begin{array}{l}\text { Fractal_sd_gtv } \\
\text { Wavelet_LLL_NGTDM_strength_gtv } \\
\text { Wavelet_HLL_GLSZM_IV_gtv } \\
\text { Wavelet_HLL_GLCM_infoCorr1_gtv } \\
\text { Wavelet_HHH_NGTDM_complexity_gtv } \\
\text { Wavelet_HLL_IH_mingradi_gtv } \\
\text { Wavelet_HLH_GLCM_infoCorr1_gtv } \\
\text { Wavelet_HLH_GLSZM_ZE_gtv } \\
\text { Wavelet_LLL_GLCM_infoCorr1_gtv } \\
\text { Wavelet_LHL_Fractal_sd_gtv }\end{array}$ & $\begin{array}{l}\text { Shape_maxDiameter2D3_lung } \\
\text { Wavelet_LHH_NGLDM_LGSDE_lung } \\
\text { Shape_volumeDensityBE_lung } \\
\text { Wavelet_LHL_Stats_max_lung } \\
\text { Wavelet_HHL_NGLDM_LGSDE_lung } \\
\text { Wavelet_HLL_Stats_median_lung } \\
\text { Wavelet_LLH_IH_cov_lung } \\
\text { Wavelet_LHH_GLCM_maxCorr_lung } \\
\text { Wavelet_LHH_Stats_mean_lung } \\
\text { Wavelet_HHH_GLDZM_LILDE_lung }\end{array}$ & $\begin{array}{l}\text { Wavelet_LLL_GLCM_inverseVar_lung } \\
\text { Wavelet_LHH_Stats_cov_gtv } \\
\text { Wavelet_LLH_IH_cov_lung } \\
\text { Wavelet_HLL_IH_kurtosis_lung } \\
\text { Stats_min_lung } \\
\text { Shape_volumeDensityBE_lung } \\
\text { Wavelet_LHH_NGLDM_LGSDE_lung } \\
\text { Wavelet_LHL_Stats_qcod_lung } \\
\text { Wavelet_HLL_Stats_min_lung } \\
\text { GLDZM_LILDE_gtv }\end{array}$ \\
\hline $\begin{array}{l}\text { Pleural } \\
\text { thickening }\end{array}$ & & $\begin{array}{l}\text { GLDZM_HILDE_lung } \\
\text { Wavelet_LLL_Stats_qcod_lung } \\
\text { Wavelet_HLH_GLDZM_LILDE_lung } \\
\text { Wavelet_HLH_Stats_median_lung } \\
\text { Wavelet_LHL_Stats_cov_lung } \\
\text { Wavelet_LLH_GLRLM_RE_lung } \\
\text { Wavelet_HLL_Stats_mean_lung } \\
\text { NGLDM_DN_lung } \\
\text { Wavelet_HLL_GLDZM_HISDE_lung } \\
\text { Wavelet_HLH_Stats_min_lung }\end{array}$ & $\begin{array}{l}\text { Wavelet_HLL_IH_skewness_gtv } \\
\text { Wavelet_LLH_GLCM_maxCorr_lung } \\
\text { Wavelet_LLL_Stats_qcod_lung } \\
\text { Shape_volumeDensityBE_lung } \\
\text { Wavelet_LLH_Stats_mean_lung } \\
\text { Wavelet_LLH_NGLDM_DV_lung } \\
\text { Wavelet_LLL_IH_qcod_lung } \\
\text { Wavelet_LHH_GLCM_maxCorr_gtv } \\
\text { Wavelet_LLL_GLCM_inverseVar_lung } \\
\text { Wavelet_HHL_Stats_min_lung }\end{array}$ \\
\hline $\begin{array}{l}\text { Satellite } \\
\text { Nodules }\end{array}$ & & $\begin{array}{l}\text { Wavelet_HHL_Stats_mean_lung } \\
\text { Wavelet_HLL_Stat_min_lung } \\
\text { Wavelet_LHH_NGLDM_LGSDE_lung } \\
\text { Wavelet_HLL_Stats_median_lung } \\
\text { Wavelet_LHH_GLDZM_DZN_lung } \\
\text { Wavelet_LHH_GLCM_infoCorr1_lung } \\
\text { Wavelet_LHH_Stats_mean_lung } \\
\text { Wavelet_HLL_GLCM_infoCorr2_lung } \\
\text { Wavelet_HLH_IH_qcod_lung } \\
\text { Wavelet_HLH_Stats_median_lung }\end{array}$ & $\begin{array}{l}\text { Wavelet_HLH_NGTDM_complexity_gtv } \\
\text { Wavelet_LLH_GLDM_LILDE_gtv } \\
\text { Wavelet_LHH_GLCM_clusShade_gtv } \\
\text { Wavelet_HLL_IH_cov_lung } \\
\text { Wavelet_LLL_GLDZM_LILDE_lung } \\
\text { Shape_flatness_gtv } \\
\text { Wavelet_HLL_GLCM_maxCorr_gtv } \\
\text { NGLDM_DV_lung } \\
\text { GLDZM_HILDE_lung } \\
\text { Wavelet_HLH_GLSZM_SZNN_lung }\end{array}$ \\
\hline
\end{tabular}

Table 8.3: Top 10 ranked radiomic features for the tumor, lung and the combination for air bronchogram, necrosis, pleural effusion, pleural thickening and satellite nodules. 



\section{Chapter 9}

Radiomics approach to predict skeletal muscle response to chemotherapy in stage IV non-small cell lung cancer

Authors

Evelyn E.C. de Jong, Karin J.C. Sanders, Timo M. Deist, Wouter van Elmpt, Arthur Jochems, Janna E. van Timmeren, Ralph T.H. Leijenaar, Juliette H.R.J. Degens, Annemie M.W.J. Schols, Anne-Marie C. Dingemans,

Philippe Lambin

In preparation 


\section{Abstract}

\section{Background}

Skeletal muscle loss associates with low quality of life, diminished treatment efficacy and poor survival rates in cancer patients. Prevention and timely treatment of muscle loss requires prediction of patients at risk. Therefore, the main goal of the present study was to assess whether baseline skeletal muscle radiomic features can predict future muscle loss. We furthermore investigated if longitudinal changes in muscle radiomic features correlate to computed tomography (CT)-derived muscle loss.

\section{Methods}

CT scans of 116 stage IV non-small cell lung cancer patients included in a randomized controlled trial (NCT01171170) were analysed. Skeletal muscle cross-sectional area and radiomic features were assessed at the third lumbar level on CT images obtained before initiation of chemotherapy and shortly after administration of the second cycle. For internal cross-validation of the radiomics modelling approach, the cohort was randomly split in a training set and validation set 100 times. A least absolute shrinkage and selection operator (LASSO) feature selection was performed. The area under the curve (AUC) was used to show model performance. This was done using radiomic features of the baseline scan as well as the difference in radiomic features between the baseline scan and the follow-up scan, so called delta radiomic features.

\section{Results}

75 Patients (65\%) exhibited loss of skeletal muscle. A total of 1298 radiomic features were extracted. After elimination of correlated features, 193 radiomic features of the baseline scan were used to construct a prediction model with muscle loss as outcome. The average AUC was $0.49(95 \% \mathrm{Cl}: 0.36,0.62)$. Differences in intensity and texture radiomic features over time were seen between patients with and without muscle loss. The AUC for delta radiomics was $0.68(95 \% \mathrm{Cl}: 0.51,0.84)$.

\section{Conclusions}

The present study shows that skeletal muscle radiomics at baseline cannot predict future muscle loss during chemotherapy in metastatic NSCLC patients. Differences in radiomic features over time might reflect myosteatosis. Future imaging analysis combined with muscle tissue analysis in patients and in experimental models is needed to unravel the biological processes linked to the radiomic features. 


\section{Introduction}

Cachexia is a frequently observed phenomenon of skeletal muscle and adipose tissue depletion among non-small cell lung cancer (NSCLC) patients $[1,2]$. The progressive loss of muscle has a devastating impact on quality of life [3] and survival rates in NSCLC patients $[2,4,5,6]$. Although both muscle and fat become depleted, there is evidence that body fat is lost more rapidly than muscle [7, 8]. Cancer may therefore shift lipid metabolism to a catabolic state, which in turn may affect skeletal muscle. In cancer cachexia depletion of subcutaneous fat is driven by increased lipolysis [9, 10]. Experimental research has shown that lipolysis generates fatty acids which are able to transport into myocytes and stimulate protein degradation [11]. Indeed, skeletal muscle of cancer patients contained more intramyocellular fat compared to age- and gender matched controls [12]. However, the exact role of intramyocellular fat and its prognostic significance in cachexia progression needs to be elucidated.

Radiomics is a method to quantitatively characterise regions of interest in medical images. The method extracts a large number of quantitative imaging features. These features describe shape, size, intensity, and texture $[13,14,15]$. The underlying idea is that medical images contain information that reflects elemental pathophysiology, which can be revealed via quantitative analyses $[16,17]$. Until now, radiomics has mainly been applied to extract tumour features in oncologic patients to visualize tumour heterogeneity or predict radiation pneumonitis $[17,18]$. The obtained features are predictive for clinical outcomes, including tumour aggressiveness and prognosis $[19,20,21]$. It is therefore argued that radiomic features can help to better understand biological and pathological processes $[16,17]$.

There are currently no methods available to identify patients at risk, which would be very helpful for timely treatment decisions. To date, analysis of muscle characteristics using radiomics has never been used to predict muscle loss. The primary goal of this exploratory study is to investigate whether baseline skeletal muscle radiomic features are different between patients who develop muscle loss and those who maintain their muscle mass after chemotherapy. We furthermore investigated if longitudinal changes in muscle radiomic features are associated to muscle loss.

\section{Material and Methods}

\section{Patient cohort}

CT scans derived from the multicentre randomized phase II trial (NVALT12 trial, NCT01171170) were investigated. In this trial the effect of nitroglycerin added to paclitaxel-carboplatin-bevacizumab on progression free survival in chemotherapy naïve stage IV non-squamous NSCLC was investigated. The methodology and results of this trial have been published previously [22].

\section{Image analysis}

CT scans made at baseline and after the second cycle of chemotherapy were used. To evaluate whether or not patients lost skeletal muscle, cross-sectional measurements of skeletal muscle areas were made on transverse images at the third lumbar level with SliceO-Matic software v5.0 (Tomovision, Montreal, Canada). One slice at the third lumbar 
level in each scan was selected for each patient. Skeletal muscle cross-sectional area was quantified on the basis of pre-established thresholds of Hounsfield units (-29 to 150). It is of note, that this delineation excludes intramuscular fat. Changes in muscle cross-sectional areas between CT scans were expressed as a percentage, which was normalized for the time interval between scans (percentage change per 100 days). A measurement error of $1.3 \%$ was adopted, based on previous reported literature $[2,4]$. Changes greater than or equal to $-1.3 \%$ were considered as 'loss of skeletal muscle', while changes less than $-1.3 \%$ were considered 'maintenance of skeletal muscle'. Additionally, the mean Hounsfield units of the muscle CSA were assessed, as a measure for muscle fat deposits. Low values reflect increased muscle fat.

Next, to evaluate radiomic features, skeletal muscle cross-sectional area was delineated at the third lumbar level using the same thresholds of Hounsfield units as described above. Now it was extended one slice in the cranial direction and one slice in the caudal direction using Mirada software (Mirada Medical, Oxford, UK), to be able to calculate three-dimensional image features. Image features were calculated on both baseline and follow-up scans, using an adapted version of Computational Environment for Radiotherapy Research extended with in-house developed radiomic image analysis software (Matlab 2014a, The Mathworks, Natick, MA). Prior to the extraction of features a grey-level discretisation using a bin width of 25 Hounsfield units was applied. To minimize the possible effect of the variation in image parameters, all scans were resampled to a voxel size of $1 \times 1 \times 3 \mathrm{~mm}^{3}$ using a cubic interpolation as recommended in Larue et al. [23].

\section{Radiomic feature selection and statistics}

Intensity and texture features were analysed. Shape and size features were excluded because the volumes of interest has been segmented manually, which might influence the outcome of these feature categories. In addition, a three-dimensional wavelet transformation was applied to the CT scan to create filtered, next to the unfiltered intensity and texture features. Features without a range (i.e. features with an exact similar value in all patients), which were not able to discriminate patients, were excluded.

Spearman's correlation coefficient $(\rho)$ was used to assess the correlation between all texture and intensity radiomic features. Feature pairs with $|\rho| \geq 0.85$ were excluded. Of each feature pair with $|\rho| \geq 0.85$, the feature that was strongest correlated to all other features was excluded. This process was repeated until no feature pair with all $|\rho| \geq 0.85$ was remaining.

To calculate to which extent the variation among the radiomic features on baseline scans is explained by muscle loss, a logistic Least Absolute Shrinkage and Selection Operator (LASSO) regression model adopting a 100-fold Monte Carlo cross-validation in Matlab 2017b was applied (The Mathworks, Natick, MA).

The cohort was randomly split in a training set (approximately 2/3) and validation set (approximately $1 / 3$ ). Patients were randomized such that the ratio between patients with and without skeletal muscle loss was similar in each group. Features in the training and validation sets were standardized by subtracting the respective mean feature value in the training set and dividing by the feature standard deviation in the training set. The logistic LASSO model was used to reduce the number of features and estimate regression coefficients for the remaining features. The model-intrinsic parameter $\lambda$ was estimated using an internal 5-fold cross-validation on the training set. The out-of-sample area 
under the curve (AUC) of the Receiver operating characteristic curve (ROC) was computed on the validation set to assess the prognostic power. This process was repeated 100 times, each time with a different randomization of the patients into training and validation sets, and the average AUC over the hundred different models was calculated. This analysis was done for the radiomic texture and intensity features of the baseline and for the absolute difference in feature values between the baseline scan and the follow-up scan (delta features). For all radiomic features that were at least selected once in the LASSO feature selection procedure the difference in feature value between patients with and without muscle loss were compared by plotting a heatmap. For the heatmap all radiomic features were normalized to have values between 0 and 1 . A hierarchical clustering was applied on the same heatmap to identify clusters of patients with different radiomic texture and intensity values.

\section{Results}

\section{Patients and characteristics}

In total 223 patients were enrolled in the randomized controlled trial. The inclusion of the present paper is based on previous analysis of change in muscle mass and its association to overall survival [6]. Therefore, one hundred three patients were excluded due to unavailability of one or both CT scans, two patients were excluded because L3 was not evaluable, one patient was excluded due to lacking overall survival (OS) data and one was excluded for insufficient quality of the scans. After exclusion, CT scans from 116 patients were eligible. The mean age was 61 years and sixty-four patients (55\%) were male.

\section{Muscle maintenance and muscle loss}

Delineations of skeletal muscle cross-sectional area made with Slice-O-Matic for crosssectional area and with Mirada for extraction of radiomic features are shown in Figure 9.1. Analysis of skeletal muscle cross-sectional area at baseline and during follow-up revealed that in the whole cohort skeletal muscle decreased with mean ( \pm standard deviation) -5.4 $\pm 14.0 \%$ per 100 days. Of those, 75 patients $(65 \%)$ exhibited loss of skeletal muscle.

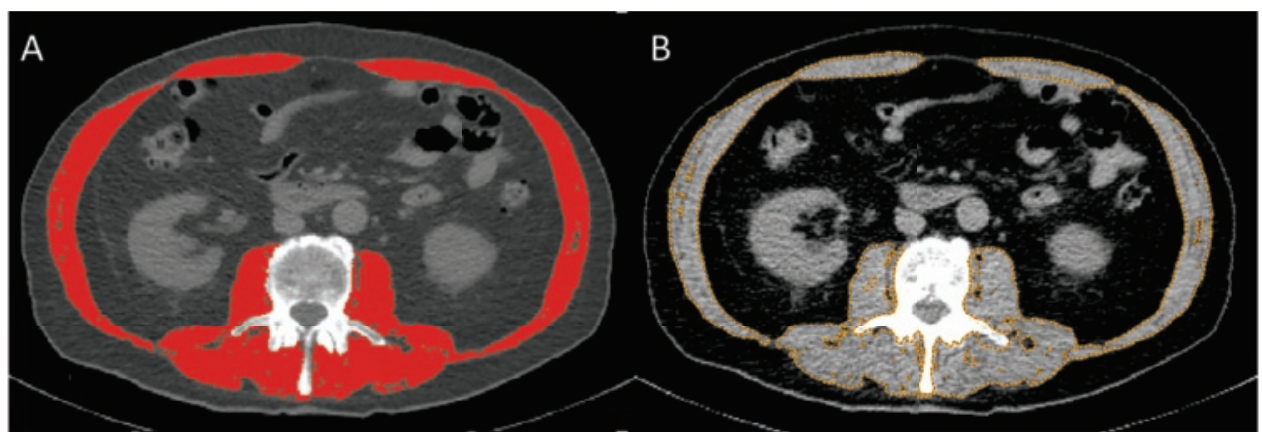

Figure 9.1: Skeletal muscle area on transverse CT images at the third lumbar level, using (a) Slice-O-Matic for evaluation of cross-sectional area and (b) Mirada software for extraction of radiomic features. 


\section{Radiomic features}

A total of 1298 radiomic features were extracted. For analysis of baseline radiomic features, those which had no range $(n=9)$ were excluded. After removing the redundant features using the spearman correlation method, 193 radiomic features, 11 unfiltered and 182 filtered, could be used for the analysis. The average AUC over 100 repetitions for radiomic features of the baseline scan, with muscle loss as outcome, is $0.49(95 \% \mathrm{Cl}: 0.36,0.62)$.

For analysis of the delta features (difference in feature values between the baseline scan and the follow-up scan), eight features without a range were excluded. After removing the redundant features using the spearman correlation method, 349 radiomic features, 33 unfiltered and 316 filtered, were used to construct an AUC. For the delta radiomic features, with muscle loss as outcome, the average AUC is 0.68 (95\% Cl: $0.51-0.84)$.

The 20 most frequently selected delta radiomics features in LASSO models for muscle loss in a 100-fold Monte Carlo cross-validation were plotted in Figure 9.2, it can be seen that features from the grey-level co-occurrence matrix (GLCM) were most often selected. Most features selected in the models were wavelet filtered features, 17 of the top 20 mostly selected features.

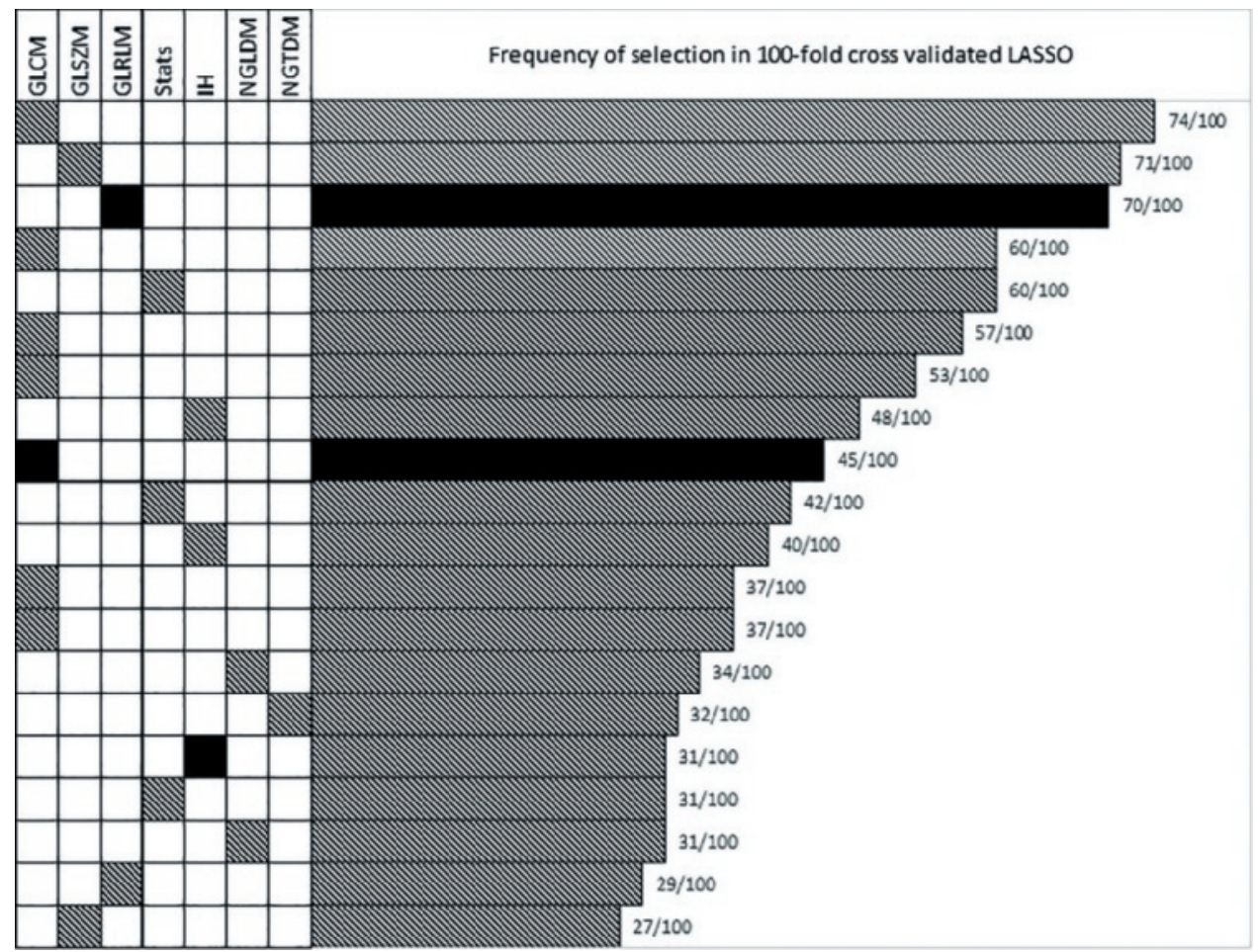

Figure 9.2: The 20 most frequently selected delta radiomics features in LASSO models for muscle loss in a 100 -fold Monte Carlo cross-validation. The solid bars represent unfiltered radiomic features and the textured bars represent wavelet filtered features.

In Figure 9.3 a heatmap is shown in which radiomic features that are selected at least once in the LASSO models, are clustered according Ward's minimum variance method. Two clusters of patients based on differences in radiomic texture and intensity values 


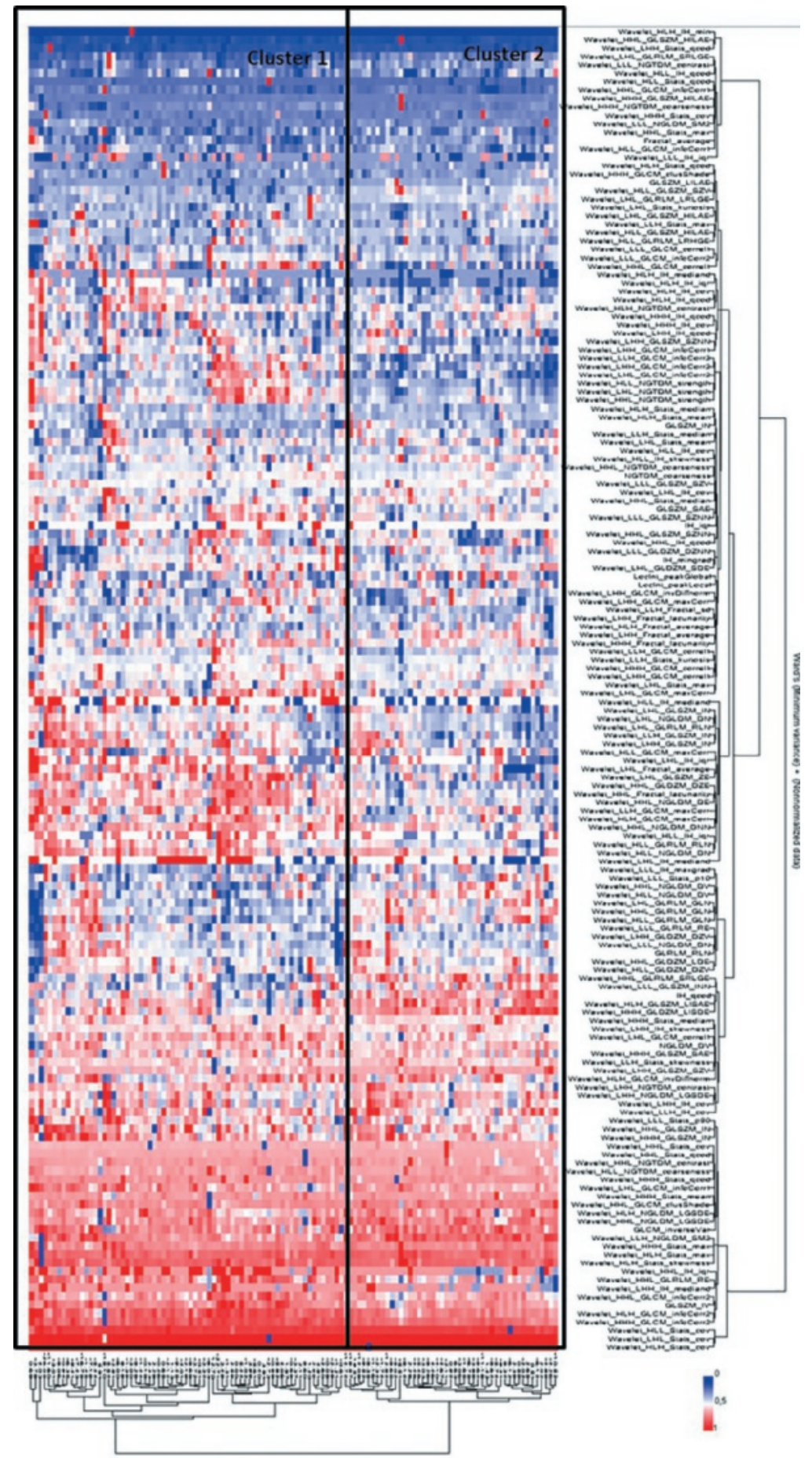

Figure 9.3: Clustered heatmap of selected delta radiomic features. 
could be defined. The clusters were similar regarding age, percentage of muscle loss, change in mean Hounsfield units, Charlson comorbidity index, treatment arm, smoking status, and overall survival. A difference was found in gender distribution, as cluster 2 contained $71 \%$ male patients compared to $44 \%$ male patients in cluster $1(p=0.004)$. We therefore evaluated potential gender differences regarding clinical response and change in body composition in the whole cohort, but found none [data not shown]. Figure 9.4 shows a heatmap in which radiomic features that are selected at least once in the LASSO models were plotted against the patients ranked in descending order of the decrease in muscle mass. The upper half of the graph shows the patients with muscle loss, the lower half of the graph the patients with a stable muscle mass. No difference in radiomic features is seen between those with maintenance of muscle and those with muscle loss. A list with the selected radiomic features in alphabetic order can be found in the Supplementary Material Table 9.1.

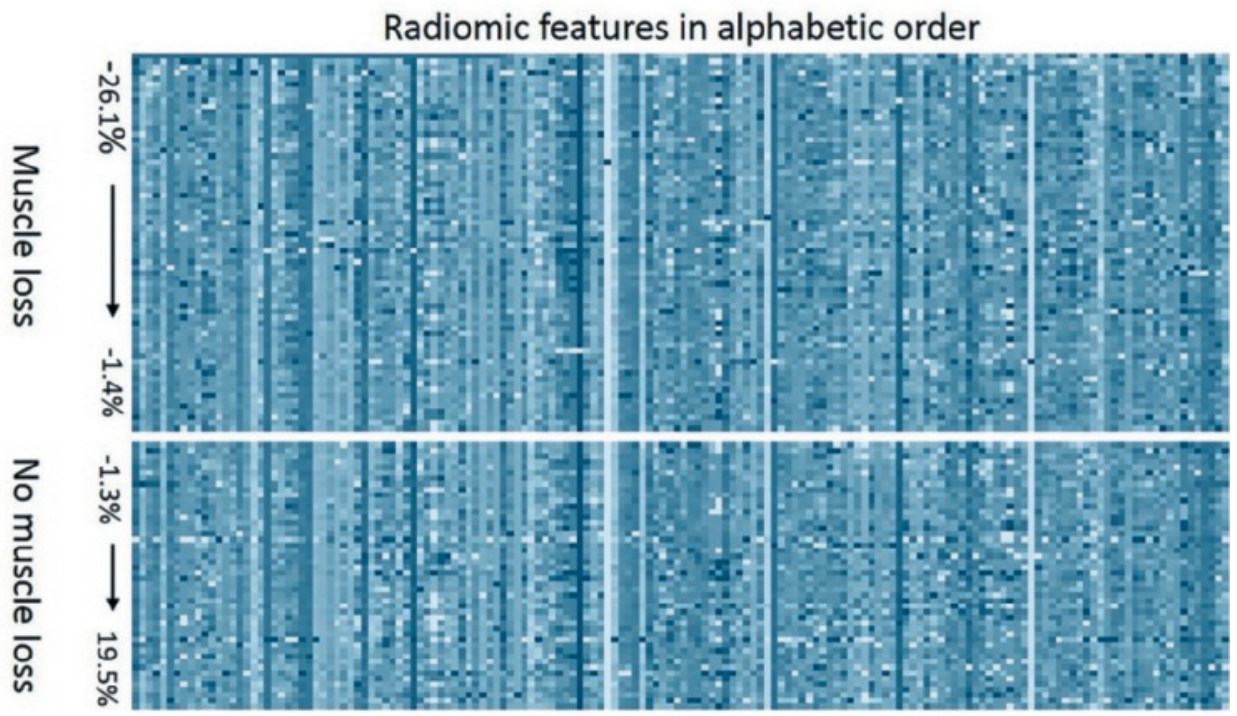

Figure 9.4: Heatmap of the normalized selected delta radiomic features in alphabetic order (list of features can be found in supplementary material). Patients are ranked according to the descending difference in muscle mass; upper half are the patients with muscle depletion, lower half patients with stable muscle mass.

\section{Discussion}

To the best of our knowledge, this is the first exploratory study evaluating the potential of skeletal muscle radiomics to predict skeletal muscle loss. Some cross-sectional studies show that baseline low CT-derived muscle mass is an important prognostic factor for OS [4, 24], which contradicts to other studies $[25,26]$. The lack of properly validated and population specific cut-off values for CT-derived low muscle mass may explain this discrepancy. We therefore were interested if radiomic features of baseline skeletal muscle are prognostic for 
longitudinal muscle loss. In this study, baseline radiomic features had no discriminatory value with regard to longitudinal skeletal muscle changes. However, longitudinal differences in radiomic features were seen between those who lost muscle and those who maintained muscle mass.

The most distinct delta radiomic feature was a feature in the grey-level co-occurrence matrix (GLCM) category. To calculate the GLCM, each pixel in an image is assigned a numerical value depending on the combination of grey-level intensity values in two neighbouring pixels. Second order statistics calculate mathematical algorithms to derive textural homogeneity, contrast, variance etc. [27]. GLCM features depend on the grey level pattern, which might implicate that patients with muscle loss develop more muscle fat deposits, leading to a more heterogeneous grey level intensity pattern (as low grey levels reflect increased intramuscular fat). Indeed analysis of muscle biopsies demonstrated that compared to controls, cancer patients exhibited increased numbers of lipid droplets in skeletal muscle. Moreover, the amount of lipid droplets increased with progression of weight loss [28]. Muscle fat depots assessed by muscle radiation attenuation on CT are associated with poor survival in cancer [4]. A phantom study showed concordance between radiation attenuation and muscle lipid content [29], therefore, reduced muscle radiation attenuation is believed to reflect fat infiltration. However, it is unclear if this radiation attenuation indicates intra- or extramyocellular lipids. Therefore, the etiology and prognostic significance of muscle lipids in cachexia progression is object for further research.

While the strength of our study comes from the well-defined randomized patient cohort, with CT scans executed according to study protocol predefined time points, there are some limitations. Pre-treatment changes in muscle mass are unknown. Patients currently identified as 'muscle maintenance', could have exhibited muscle loss prior to the first CT scan, which may influence radiomic features. The NVALT12 trial is a multicentre study. Consequently, the CT scans included in this analysis are performed in different hospitals on different CT scanners with possible different scan protocols. We showed in a phantom study that slice thickness variability influences radiomic features. However, this variability in radiomic feature values can be reduced by resampling to a standardized voxel size. In addition, it was shown that different exposures do not influence the radiomic feature values [23]. Although most CT scans included in our analysis were low dose, some CT scans were performed with use of contrast. The influence of CT contrast on radiomic features is still unclear.

In conclusion, the present study shows that baseline skeletal muscle radiomics is not able to predict muscle loss in metastatic NSCLC patients. Nevertheless, longitudinal differences in texture muscle radiomic features can distinguish patients with muscle loss from patients without muscle loss. Future research in experimental models and human radiomics combined with muscle tissue analysis is required to unravel the biological processes linked to the radiomic features. 


\section{References}

[1] K. Fearon et al. "Definition and classification of cancer cachexia: an international consensus". In: Lancet Oncol 12.5 (2011). The Lancet. Oncology, pp. 489-95. DOI: $10.1016 /$ s1470-2045(10)70218-7.

[2] C. M. Prado et al. "Prevalence and clinical implications of sarcopenic obesity in patients with solid tumours of the respiratory and gastrointestinal tracts: a population-based study". In: Lancet Oncol 9.7 (2008). The Lancet. Oncology, pp. 629-35. DOI: 10.1016/s1470-2045(08)70153-0.

[3] A. Bye et al. "Muscle mass and association to quality of life in non-small cell lung cancer patients". In: J Cachexia Sarcopenia Muscle 8.5 (2017). Journal of cachexia, sarcopenia and muscle, pp. 759-767. DOI: 10.1002/jcsm.12206.

[4] L. Martin et al. "Cancer cachexia in the age of obesity: skeletal muscle depletion is a powerful prognostic factor, independent of body mass index". In: J Clin Oncol 31.12 (2013). Journal of clinical oncology : official journal of the American Society of Clinical Oncology, pp. 1539-47. DOI: 10.1200/jco.2012.45.2722.

[5] G. B. Stene et al. "Changes in skeletal muscle mass during palliative chemotherapy in patients with advanced lung cancer". In: Acta Oncol 54.3 (2015). Acta oncologica (Stockholm, Sweden), pp. 340-8. DOI: 10.3109/0284186x.2014.953259.

[6] J. Degens et al. "P2.01-048 Early Changes in Body Composition in Metastatic NonSmall Cell Lung Cancer (NSCLC) Are Predictive for Poor Overall Survival". In: Journal of Thoracic Oncology 12.11 (2017), S2087-S2088. DOI: 10.1016/j.jtho. 2017.09.1150. URL: https://doi.org/10.1016/j.jtho.2017.09.1150.

[7] M. Fouladiun et al. Body composition and time course changes in regional distribution of fat and lean tissue in unselected cancer patients on palliative care-correlations with food intake, metabolism, exercise capacity, and hormones. Cancer. 2005.

[8] T. Agustsson et al. Adipose tissue volume is decreased in recently diagnosed cancer patients with cachexia. Nutrition (Burbank, Los Angeles County, Calif.) 2012.

[9] M. Ryden et al. Lipolysis-not inflammation, cell death, or lipogenesis-is involved in adipose tissue loss in cancer cachexia. Cancer. 2008.

[10] T. Agustsson et al. Mechanism of increased lipolysis in cancer cachexia. Cancer research. 2007.

[11] Q. Zhou et al. Evidence for adipose-muscle cross talk: opposing regulation of muscle proteolysis by adiponectin and Fatty acids. Endocrinology. 2007.

[12] M. A. Weber et al. Morphology, metabolism, microcirculation, and strength of skeletal muscles in cancer-related cachexia. Acta oncologica (Stockholm, Sweden). 2009.

[13] P. Lambin et al. "Predicting outcomes in radiation oncology-multifactorial decision support systems". In: Nat Rev Clin Oncol 10.1 (2013). Nature reviews. Clinical oncology, pp. 27-40. DOI: 10.1038/nrclinonc.2012.196.

[14] P. Lambin et al. "Radiomics: extracting more information from medical images using advanced feature analysis". In: Eur J Cancer 48.4 (2012). European journal of cancer (Oxford, England : 1990), pp. 441-6. DOI: 10.1016/j.ejca.2011.11.036. 
[15] V. Kumar et al. "Radiomics: the process and the challenges". In: Magn Reson Imaging 30.9 (2012). Magnetic resonance imaging, pp. 1234-48. DOI: 10.1016/j.mri.2012.06.010.

[16] P. Grossmann et al. "Defining the biological basis of radiomic phenotypes in lung cancer". In: Elife 6 (2017). eLife. DOI: 10.7554/eLife.23421.

[17] H. J. Aerts et al. "Decoding tumour phenotype by noninvasive imaging using a quantitative radiomics approach". In: Nat Commun 5 (2014). Nature communications, p. 4006. DOI: $10.1038 /$ ncomms5006.

[18] S. P. Krafft et al. "The utility of quantitative ct radiomics features for improved prediction of radiation pneumonitis". In: Med Phys (2018). Medical physics. DOI: 10.1002/mp. 13150.

[19] J. R. Ferreira Junior et al. "Radiomics-based features for pattern recognition of lung cancer histopathology and metastases". In: Comput Methods Programs Biomed 159 (2018). Computer methods and programs in biomedicine, pp. 23-30. DOI: 10.1016/ j.cmpb.2018.02.015.

[20] T. P. Coroller et al. "Radiomic phenotype features predict pathological response in non-small cell lung cancer". In: Radiother Oncol 119.3 (2016). Radiotherapy and oncology : journal of the European Society for Therapeutic Radiology and Oncology, pp. 480-6. DOI: 10.1016/j.radonc.2016.04.004.

[21] S. A. Mattonen et al. "Imaging texture analysis for automated prediction of lung cancer recurrence after stereotactic radiotherapy". In: J Med Imaging (Bellingham) 2.4 (2015). Journal of medical imaging (Bellingham, Wash.), p. 041010. DOI: 10. 1117/1.jmi.2.4.041010.

[22] E. E. de Jong et al. "[18F]FDG PET/CT-based response assessment of stage IV non-small cell lung cancer treated with paclitaxel-carboplatin-bevacizumab with or without nitroglycerin patches". In: Eur J Nucl Med Mol Imaging 44.1 (2017). European journal of nuclear medicine and molecular imaging, pp. 8-16. DOI: 10.1007/s00259-016-3498-y.

[23] R. T. H. M. Larue et al. "Influence of gray level discretization on radiomic feature stability for different CT scanners, tube currents and slice thicknesses: a comprehensive phantom study". In: Acta Oncol In press (2017), p. 11. DOI: 10.1080/0284186X.2017.1351624.

[24] Y. Suzuki et al. "Clinical implications of sarcopenia in patients undergoing complete resection for early non-small cell lung cancer". In: Lung Cancer 101 (2016). Lung cancer (Amsterdam, Netherlands), pp. 92-97. DOI: 10.1016/j.lungcan.2016.08.007.

[25] I. J. Rutten et al. "Loss of skeletal muscle during neoadjuvant chemotherapy is related to decreased survival in ovarian cancer patients". In: J Cachexia Sarcopenia Muscle 7.4 (2016). Journal of cachexia, sarcopenia and muscle, pp. 458-66. DOI: $10.1002 /$ jcsm. 12107.

[26] B. Sjoblom et al. "Skeletal muscle radiodensity is prognostic for survival in patients with advanced non-small cell lung cancer". In: Clin Nutr 35.6 (2016). Clinical nutrition (Edinburgh, Scotland), pp. 1386-1393. DOI: 10.1016/j.clnu.2016.03.010.

[27] R. M. Haralick, K. Shanmugam, and I. Dinstein. "Textural Features for Image Classification". In: IEEE Transactions on Systems, Man, and Cybernetics SMC-3.6 (1973), pp. 610-621. DOI: 10.1109/TSMC.1973.4309314. 
[28] N. A. Stephens et al. Intramyocellular lipid droplets increase with progression of cachexia in cancer patients. Journal of cachexia, sarcopenia and muscle. 2011.

[29] B. H. Goodpaster et al. Skeletal muscle attenuation determined by computed tomography is associated with skeletal muscle lipid content. Journal of applied physiology (Bethesda, Md. : 1985). 2000. 


\section{Supplementary Material}

1. Fractal_average

2. GLCM_inverseVar

3. GLRLM_RLN

4. GLSZM-IN

5. GLSZM_IV

6. GLSZM_LILAE

7. GLSZM_SAE

8. IH_iqr

9. IH_mingrad

10. IH_qcod

11. Loclnt_peakGlobal

12. Loclnt_peakLocal

13. NGLDM

14. NGTDM_coarseness

15. Wavelet_HHH_Fractal_lacunarity

16. Wavelet_HHH_GLCM_clusShade

17. Wavelet_HHH_GLCM_correl1

18. Wavelet_HHH_GLCM_infoCorr2

19. Wavelet_HHH_GLDZM̄_LISDE

20. Wavelet_HHH_GLSZM_HILAE

21. Wavelet_HHH_GLSZM_IN

22. Wavelet_HHH_GLSZM_SAE

23. Wavelet_HHH_IH_cov

24. Wavelet_HHH_IH_qcod

25. Wavelet_HHH_NGTDM_coarseness

26. Wavelet_HHH_Stats_cov

27. Wavelet_HHH_Stats_max

28. Wavelet_HHH_Stats_mean

29. Wavelet_HHH_Stats_median

30. Wavelet_HHH_Stats_qcod

31. Wavelet_HHL_Fractal_lacunarity

32. Wavelet_HHL_GLCM_clusShade

33. Wavelet_HHL_GLCM_correl1

34. Wavelet_HHL_GLCM_infoCorr1

35. Wavelet_HHL_GLCM_infoCorr2

36. Wavelet_HHL_GLDZM_DZE

37. Wavelet_HHL_GLDZM_LDE

38. Wavelet_HHL_GLRLM_GLN

39. Wavelet_HHL_GLRLM_RE

40. Wavelet_HHL_GLRLM_SRLGE

41. Wavelet_HHL_GLSZM_HILAE

42. Wavelet_HHL_GLSZM_IN

43. Wavelet_HHL_GLSZM_SZNN

44. Wavelet_HHL_IH_iqr

45. Wavelet_HHL_IH_qcod

46. Wavelet_HHL_NGLDM_DE

47. Wavelet_HHL_NGLDM_DNN

48. Wavelet_HHL_NGLDM_DV

49. Wavelet_HHL_NGLDM_LGSDE

50. Wavelet_HHL_NGTDM_coarseness

51. Wavelet_HHL_NGTDM_contrast

52. Wavelet_HHL_NGTDM_strength

53. Wavelet_HHL_Stats_cov

54. Wavelet_HHL_Stats_max

55. Wavelet_HHL_Stats_median

56. Wavelet_HHL_Stats_qcod

57. Wavelet_HLH_Fractal_average

58. Wavelet_HLH_GLCM_infoCorr2
59. Wavelet_HLH_GLCM_invDiffnorm

60. Wavelet_HLH_GLCM_maxCorr

61. Wavelet_HLH_GLSZM_LISAE

62. Wavelet_HLH_IH_cov

63. Wavelet_HLH_IH_iqr

64. Wavelet_HLH_IH_mediand

65. Wavelet_HLH_IH_min

66. Wavelet_HLH_IH_qcod

67. Wavelet_HLH_NGLDM_LGSDE

68. Wavelet_HLH_NGTDM_contrast

69. Wavelet_HLH_Stats_cov

70. Wavelet HLH Stats max

71. Wavelet_HLH_Stats_mean

72. Wavelet_HLH_Stats_median

73. Wavelet_HLH_Stats_qcod

74. Wavelet_HLH_Stats_skewness

75. Wavelet_HLL_GLCM_infoCorr1

76. Wavelet_HLL_GLCM_maxCorr

77. Wavelet_HLL_GLDZM̄_DZV

78. Wavelet_HLL_GLRLM_GLN

79. Wavelet_HLL_GLRLM_LRHGE

80. Wavelet_HLL_GLRLM_RLN

81. Wavelet_HLL_GLSZM_HILAE

82. Wavelet_HLL_GLSZM_SZV

83. Wavelet_HLL_IH_cov

84. Wavelet_HLL_IH_iqr

85. Wavelet_HLL_IH_mediand

86. Wavelet_HLL_IH_qcod

87. Wavelet_HLL_IH_skewness

88. Wavelet_HLL_NGLDM_DN

89. Wavelet_HLL_NGLDM_DV

90. Wavelet_HLL_NGTDM_coarseness

91. Wavelet_HLL_NGTDM_strength

92. Wavelet_HLL_Stats_cov

93. Wavelet_HLL_Stats_qcod

94. Wavelet_LHH_Fractal_average

95. Wavelet_LHH_Fractal_lacunarity

96. Wavelet_LHH_GLCM_correl1

97. Wavelet_LHH_GLCM_infoCorr1

98. Wavelet_LHH_GLCM_infoCorr2

99. Wavelet_LHH_GLCM_invDiffnorm

100. Wavelet_LHH_GLCM_maxCorr

101. Wavelet_LHH_GLDZM

102. Wavelet LHH GLSZM IN

103. Wavelet_LHH_GLSZM_SZNN

104. Wavelet_LHH_GLSZM_SZV

105. Wavelet_LHH_IH_cov

106. Wavelet_LHH_IH_mediand

107. Wavelet_LHH_IH_qcod

108. Wavelet_LHH_IH_skewness

109. Wavelet_LHH_NGLDM_LGSDE

110. Wavelet_LHH_NGTDM_contrast

111. Wavelet_LHH_Stats_qcod

112. Wavelet_LHL_Fractal_average

113. Wavelet_LHL_GLCM_correl1

114. Wavelet_LHL_GLCM_infoCorr1

115. Wavelet_LHL_GLCM_infoCorr2

116. Wavelet_LHL_GLCM_maxCorr

117. Wavelet_LHL_GLDZM̄_SDE

118. Wavelet_LHL_GLRLM_GLN 
119. Wavelet_LHL_GLRLM_LRLGE

120. Wavelet_LHL_GLRLM_RLN

121. Wavelet_LHL_GLRLM_SRLGE

122. Wavelet_LHL_GLSZM_HILAE

123. Wavelet_LHL_GLSZM_IN

124. Wavelet_LHL_GLSZM_ZE

125. Wavelet_LHL_IH_cov

126. Wavelet_LHL_IH_iqr

127. Wavelet_LHL_IH_mediand

128. Wavelet_LHL_NGLDM_DN

129. Wavelet_LHL_NGTDM_strength

130. Wavelet_LHL_Stats_cov

131. Wavelet_LHL_Stats_kurtosis

132. Wavelet_LHL_Stats_max

133. Wavelet_LHL_Stats_mean

134. Wavelet_LLH_Fractal_sd

135. Wavelet_LLH_GLCM_correl1

136. Wavelet_LLH_GLCM_infoCorr2

137. Wavelet_LLH_GLCM_maxCorr

138. Wavelet_LLH_GLSZM_IN
139. Wavelet_LLH_IH_cov

140. Wavelet_LLH_NGLDM_SM2

141. Wavelet_LLH_Stats_kurtosis

142. Wavelet_LLH_Stats_max

143. Wavelet_LLH_Stats_median

144. Wavelet_LLH_Stats_skewness

145. Wavelet_LLL_GLCM_correl1

146. Wavelet_LLL_GLCM_infoCorr2

147. Wavelet_LLL_GLDZM__DZNN

148. Wavelet_LLL_GLRLM_RE

149. Wavelet_LLL_GLSZM_INN

150. Wavelet_LLL_GLSZM_SZNN

151. Wavelet_LLL_GLSZM_SZV

152. Wavelet_LLL_IH_iqr

153. Wavelet_LLL_IH_maxgrad

154. Wavelet_LLL_NGLDM_DN

155. Wavelet_LLL_NGLDM_SM2

156. Wavelet_LLL_NGTDM_contrast

157. Wavelet_LLL_Stats_p10

158. Wavelet_LLL_Stats_p90

Table 9.1 Selected radiomic features in alphabetic order 

Discussion 

Chapter 10

General discussion and future perspectives 


\section{Imaging in non-small cell lung cancer}

Imaging plays a crucial role in the diagnosis, staging, treatment and follow-up of lung cancer. Imaging modalities used in routine daily care in lung cancer are computed tomography (CT), ${ }^{18} \mathrm{~F}$-fluodeoxyglucose positron emission tomography (FDG-PET), magnetic resonance imaging (MRI) and ultrasound. A PET/CT scan has a higher sensitivity than a CT scan in the detection of malignancies, however at the cost of a lower specificity [1]. Next to that, the higher sensitivity of the PET does not always have therapeutic consequences, e.g. if the PET scan detects more metastases compared to the $C T$, the patient is still diagnosed with a stage IV disease and its intended treatment [1]. While PET/MR has a higher sensitivity than PET/CT in non-small cell lung cancer (NSCLC), this does also not always lead to a different treatment. A recent study showed that ${ }^{18} \mathrm{~F}$-FDG PET/MR and ${ }^{18} \mathrm{~F}$-FDG PET/CT agreed $100 \%$ on the T-stage and that there were no significant differences between both modalities for the detection of lymph node metastases[2].

\section{Response assessment using CT and PET}

A variety of approaches have been developed to measure treatment response, starting with the World Health Organization (WHO) criteria and continuing to Response Criteria In Solid Tumors (RECIST) and RECIST $1.1[3,4]$. These criteria refer to an anatomical decrease in tumor diameter. However, this response assessment must be viewed with some caution when assessing treatment outcomes in therapies that may be more cytostatic than cytotoxic. With cytostatic therapies, lack of progression may be associated with an improvement in outcome, even in the absence of major shrinkage of tumors [5]. Therefore, newer metrics such as PET-based imaging biomarkers may be more informative [6]. Currently, two sets of treatment response criteria for PET are available: European Organization for Research and Treatment of Cancer (EORTC) [7] and PET response criteria in solid tumors (PERCIST) [8]. Next to quantitative response criteria, there also exist visual response criteria. The first visual response criteria for PET were published in 2003 [9]. In 2012 visual response criteria specific for lymphoma were published [10]. In a recent study, the quantitative PET response criteria of the EORTC and PERCIST, were compared to the visual response criteria of Peter Mac and Deauville in NSCLC [11]. Although all four response criteria were highly predictive for overall survival, the visual response criteria showed a better discrimination between complete metabolic responders and non-responders [11]. The EORTC criteria and PERCIST showed similar results. Another recent study showed that the EORTC response criteria and the PERCIST criteria gave similar prognostic stratification [12]. Therefore, for PET response assessment the visual response criteria of Deauville or Peter Mac are preferred over the quantitative response criteria of the EORTC and PERCIST. A recent study compared the anatomical CT-based response criteria RECIST with the metabolic PET-based response criteria PERCIST [13]. They showed that in only $45 \%$ of the patients there was concordance between both response criteria, in which PERCIST response criteria had a higher agreement with pathological response (94\%) than RECIST response assessment (72\%). Nevertheless, PET response assessment had a higher sensitivity $(77.8 \%)$ and specificity $(75 \%)$ than CT response assessment $(66.7 \%$ and $70.5 \%$, respectively) [13]. In the Dutch multicenter randomized phase II clinical trial NVALT12 (NCT01171170), patients were randomized between 
paclitaxel-carboplatin-bevacizumab with or without the addition of nitroglycerin (NTG), to investigate the added value of NTG on progression free survival (PFS) [14]. For all patients that had a baseline PET/CT-scan a second PET/CT-scan after three weeks was performed. In this thesis we compared CT-based response assessment and PET-based response assessment for patients included in the NVALT12 study, on the primary tumor only, while RECIST and PERCIST are based on the total tumor load. Early metabolic response was defined as a $30 \%$ decrease in the maximum standardized uptake value $\left(\mathrm{SUV}_{\text {max }}\right), \mathrm{SUV}_{\text {mean }}$ or $\mathrm{SUV}_{\text {peak }}$ relative to the baseline scan. The early anatomic response was defined as a $30 \%$ decrease in CT diameter or CT volume relative to the baseline scan. The comparison of $30 \%$ CT-based and $30 \%$ PET-based response assessment performed after 3 weeks (median time interval between scans 42 days) with the RECIST analysis performed in week 6 (median time interval between scans 56 days) showed that the RECIST analysis defined more patients as responders than the $30 \%$ CT-based analysis performed after 3 weeks. This can be caused by the difference in timing, but also due to the fact that for the $30 \%$ CT-based analysis only one lesion was measured while in RECIST multiple lesions were measured. The $30 \%$ PET-based response assessment performed after 3 weeks showed more responders than the RECIST analysis, which is probably caused by decreased perfusion due to the bevacizumab treatment, which led to a decrease in FDG uptake for both treatment arms. In another study, in which PET-based response assessment in NSCLC patients treated with erlotinib and bevacizumab was performed after 3 weeks of treatment, showed that five of nine patients were classified as responders by CT while six of nine were classified as responders by ${ }^{18}$ F-FDG PET [15], which is in line with the study that compared RECIST response assessment with PERCIST response assessment. In the same study, patients with a metabolic response (decrease in SUV > $20 \%$ ) at week 3 had a longer PFS than those without (9.7 months vs. 2.8 months), while patients with a response on CT at week 3 did not have a significantly longer PFS than those without. These two findings combined showed that PET may be able to show treatment response earlier than CT. In the study of Dingemans et al. [15], ${ }^{18} \mathrm{~F}-\mathrm{FDG}$ PET scans were performed before bevacizumab infusion, while in the NVALT12 study the ${ }^{18}$ F-FDG PET scan was performed shortly after bevacizumab infusion. This might have impacted the uptake of FDG. Response assessment using ${ }^{18}$ F-FDG PET can detect responders earlier (from three weeks after the start of chemotherapy treatment) than a CT based response assessment, however caution needs to be taken with treatments that influence tumor perfusion, while this may lead to false positives. While tumors, lymph nodes and metastases can respond differently on treatment, it is recommended to perform PERCIST response assessment of total tumor load instead of only focusing on the primary tumor. Ultimately, it would be even better to select patients before the start of treatment. Outcome prediction models (either prognostic or predictive) can be used to up front estimate if a patient will respond on a specific treatment. Prognostic models give information about a likely outcome, for example survival, independent of treatment received [16]. Medical imaging can as well be used in predictive models by changing therapy options during the course of treatment. Images should therefore be processed in such a way that imaging biomarkers can be extracted and interpreted for such purpose. 


\section{Image biomarkers}

\section{Simple image features}

An imaging biomarker is a measured indicator of normal biological processes, pathogenic processes or responses to treatment [17]. Simple PET derived metrics like the SUV $V_{\text {max }}$, $\mathrm{SUV}_{\text {mean }}, \mathrm{SUV}_{\text {peak }}$ or the total lesion glycolysis have been described to determine the prognosis of NSCLC patients $[18,19,20]$. Many studies have shown that by using these ${ }^{18}$ F-FDG PET SUV metrics, response to chemotherapy can be assessed as early as three weeks after the start of therapy [21, 22, 23, 24, 25]. In the NVALT12 trial, the added value of NTG to paclitaxel-carboplatin-bevacizumab was investigated. Many factors influence tumor resistance and response to treatment. Tumor hypoxia is a negative prognostic factor in lung cancer and seems to be related to treatment resistance. Both at cellular level as well as on a population level, a negative correlation between perfusion $C T$ and hypoxia PET is described [26, 27], suggesting that hypoxia is negatively correlated to tumor blood flow. NTG, which is a vasodilator, increases tumor blood flow, consequently augments antitumor drug delivery and inhibits hypoxia inducible factor (HIF- $1 \alpha$ ). When hypoxia is negatively correlated to tumor blood flow and NTG increases tumor perfusion, this could translate into a change in FDG uptake [28]. The feasibility of PET response assessment to paclitaxel-carboplatin-bevacizumab treatment with and without NTG is investigated in this thesis. Changes in PET derived imaging parameters such as $S U V_{\max }, \mathrm{SUV}_{\text {peak }}, \mathrm{SUV}_{\text {mean }}$ and the total lesion glycolysis (TLG: SUV $_{\text {mean }} \times$ metabolic tumor volume) between the baseline and the second scan, were not significantly different, for the NTG group compared to the control group. In a previous study by Matthews et al. [29], the administration of nitric oxide (NO) donating drugs decreased hypoxia-induced resistance to anticancer drugs in cancer cell lines. In our study, this could not be confirmed based on PET analysis. This could be due to a lower NTG dose or to an interference with bevacizumab. However from recent studies, it is known that FDG is only a moderate surrogate for hypoxia [30]. The study of Zegers et al. [31] showed that $42 \pm 21 \%$ of the primary tumor volume has a high FDG uptake of which $10 \pm 12 \%$ is hypoxic and that $3 \%$ of the primary tumor volume outside the high FDG uptake volume is hypoxic depicted by ${ }^{18} \mathrm{~F}-\mathrm{HX} 4$. For the NVALT 12 study, we therefore only quantified/correlated the effects of NTG on tumor metabolism and survival but not on hypoxia directly. The NVALT12 study showed that the addition of NTG to paclitaxel-carboplatin-bevacizumab therapy did not improve PFS or overall survival (OS). Surprisingly, we could show that nearly all patients, irrespective of the addition of NTG, had a major decrease in FDG uptake in the ${ }^{18}$ F-FDG PET scan performed after 3 weeks. Importantly, this 18F FDG PET scan was acquired within 3 days after the administration of the second cycle of chemotherapy. A study by van der Veldt et al. [32] showed that bevacizumab reduces tumor perfusion and ${ }^{11} \mathrm{C}$ docetaxel uptake in NSCLC, which was accompanied by rapid reduction in circulating levels of VEGF. This decrease in tumor blood flow after bevacizumab administration may explain the lower uptake of FDG in the tumor. Consequently, our results do not exclude the possibility that NTG decreases hypoxia. To confirm this effect a hypoxia PET imaging study needs to be performed. In conclusion, because FDG may not be a good surrogate for hypoxia, studies investigating the effect of hypoxia treatment preferably use hypoxia tracers as ${ }^{18} \mathrm{~F}-\mathrm{HX} 4,{ }^{18} \mathrm{~F}$-fluoroazomycinarabinoside (FAZA) or ${ }^{18}$ F-fluoromisonidazole (MISO). When ${ }^{18} \mathrm{~F}-\mathrm{FDG}-\mathrm{PET}$ is used for response assessment of paclitaxel-carboplatin-bevacizumab treatment the ${ }^{18}$ F-FDG PET scan should be performed minimal five days after bevacizumab infusion. For response 
assessment it is advised to use $\mathrm{SUV}_{\text {peak }}$ or $\mathrm{SUV}_{\text {mean }}$ as they show less variability compared to $\mathrm{SUV}_{\max }$ [33]. Potentially a more detailed investigation of the full image dataset and tumors complemented with more complex image biomarkers can give additional information about tumor heterogeneity and therefore aggressiveness of the tumor.

\section{Semantic features}

Next to simple SUV metrics, also visually scored image characteristics, so called semantic features, may yield prognostic information. A recent study showed that semantic features could predict disease progression [34]. A study in sarcoma patients showed that simple SUV metrics combined with semantic features predicts treatment outcomes [35]. A decreased volumetric muscle CT attenuation was associated with increased distant cancer recurrence, and increased PET SUV of muscle was associated with decreased overall survival. Muscles with on average higher HUs may have less intramuscular fat. A higher level of IMAT has been associated with lower muscle quality and a worse prognosis. Patients with lower levels of IMAT are therefore suggested to have a better outcome. The SUV measures were associated with lower serum albumin and lower hemoglobin, which shows that PET can potentially be a biomarker for serum markers related to cachexia [35]. A study of Segal et al. [36] has shown that non-invasive imaging can decode genomic activity of human liver cancers. A study of Rizzo et al. [37] has shown that semantic features, like lesion cavitation, air bronchogram and pleural effusion are associated with alterations of epidermal growth factor receptor (EGFR), anaplastic lymphoma kinase (ALK) and Kirsten rat sarcoma (KRAS) genes. Current methods of molecular profiling require invasive biopsies for tissue procurement, which may limits the routinely use, especially in repeated testing. Next to that, it provides only a sample of a usually heterogeneous lesion at a specific time point. While imaging can reflect the dynamic status of tissue, it can be helpful predicting gene expression patterns [36]. However, these semantic features are scored by visual assessment of radiologists and are therefore inter-observer dependent. Therefore, we investigated if there are correlations between semantic features and quantitative radiomic features. A study of Yip et al. [38] already looked into possible correlations between semantic features and radiomic features of the tumor. Like Yip et al., we confirmed that there is an association between air bronchogram and the tumor radiomic feature "shape sphericity". Additionally, we found overlap of the non-redundant features by Yip et al., but also some other radiomic features that were not significant in the previous study. This can be due to a different number of events per semantic feature in the both datasets, but highlights the possibility of using quantitative radiomic based features to describe visual semantic features.

We also showed that some semantic features are lesion specific (e.g. cavitation and necrosis), while other semantic features describe lung characteristics (e.g. pleural effusion and satellite nodules). As the univariate analysis clearly showed, as expected, that tumor radiomic features contain information about tumor semantic features (e.g. density, air bronchogram, necrosis), the lung radiomic features contain information about lung semantic features (e.g. pleural contact and pleural effusion). To find these correlations is not straightforward, for example no radiomic feature contained information to describe calcifications, but this might be also due to the fact that only $12 \%$ of the patients of the training cohort and $3 \%$ of the patients of the validation cohort had calcifications, and also that those calcifications were visually hard to detect. For validation of models, it is therefore important that in both cohorts the number of events 
are large enough. A study of Collins et al. [39] showed that the minimum number of events is 100 and ideally, 200 or more events are required. Current datasets are hence too small to accurately classify rare events, larger datasets are needed for model building.

Our multivariate analysis showed that a combination of tumor radiomic features could detect the semantic features air bronchogram, necrosis and pleural effusion. A combination of lung radiomic features detected the semantic features pleural thickening, satellite nodules and pleural effusion. Comparing the univariate results with the multivariate results showed that airbronchogram, necrosis and pleural effusion can be detected using one, as well as, using a combination of radiomic features of the tumor, and pleural effusion can be detected, using one, or a combination of radiomic features of the lung. Although, the radiomic features in the top ten highest rank in the multivariate analysis are not significant in the univariate analysis. The difference between the results of the multivariate analysis and the univariate analysis may be explained by the fact that in the multivariate analysis also interactions between radiomic features are included. Next to that, the results in the univariate analysis most likely overfitted, due to a high number of radiomic features and a small number of patients, which is corrected for in the multivariate analysis by performing bootstrapping techniques [40].

In conclusion, correlations between semantic features and radiomic features may play a role to better understand biologic and pathological processes or allow for an early and objective classification of these processes, which in the future can lead to radiomic features that represent genetic alterations or pathological processes, an example is shown in Figure 10.1. Large datasets with genetic profiling not only in tumors but as well in lymph nodes metastases and distant metastases are needed for image biomarker studies in developing prognostic models. Next to that, correlations between radiomic features and biology need to be investigated, in the next step then genetic profiling may be correlated or complemented with radiomic features to evolve into a non-invasive characterization of the lesion investigated.

\section{Radiomic image features}

Radiomics is a quantitative method to determine the phenotype of a tumor. Although, a variety of applications of radiomics is currently investigated, there are many methodological issues that need to be solved, before the larger potential of radiomics is fully utilized. Every step in the radiomics process, as shown in Figure 10.2, has its own challenges.

Many studies have already shown the potential value of using radiomic features in prognostic models $[43,44,45]$. One of the first studies showing the potential of radiomics in lung cancer is the study of Aerts et al. [46] from 2014. First, they selected stable features from 440 radiomic features using a test-retest and a multiple delineation dataset. The 100 most stable features contained features from the statistics group, the shape group, the texture group and from the filtered texture group. The single best performing radiomic feature from each group, were combined in a prognostic model. This model was externally validated in a cohort of 225 stage I-III NSCLC patients. Next to that, was shown that the model was also prognostic in two independent cohorts of more than 200 head and neck cancer patients. The radiomic model performed better than TNM-staging in the lung validation dataset. And by combining the radiomic model with TNM-staging model performance increased in all datasets. In this thesis, the applicability of the signature was tested in a cohort of 195 stage IV NSCLC patients. The signature showed prognostic value in the stage IV NSCLC cohort, although the c-index was less prominent (c-index 

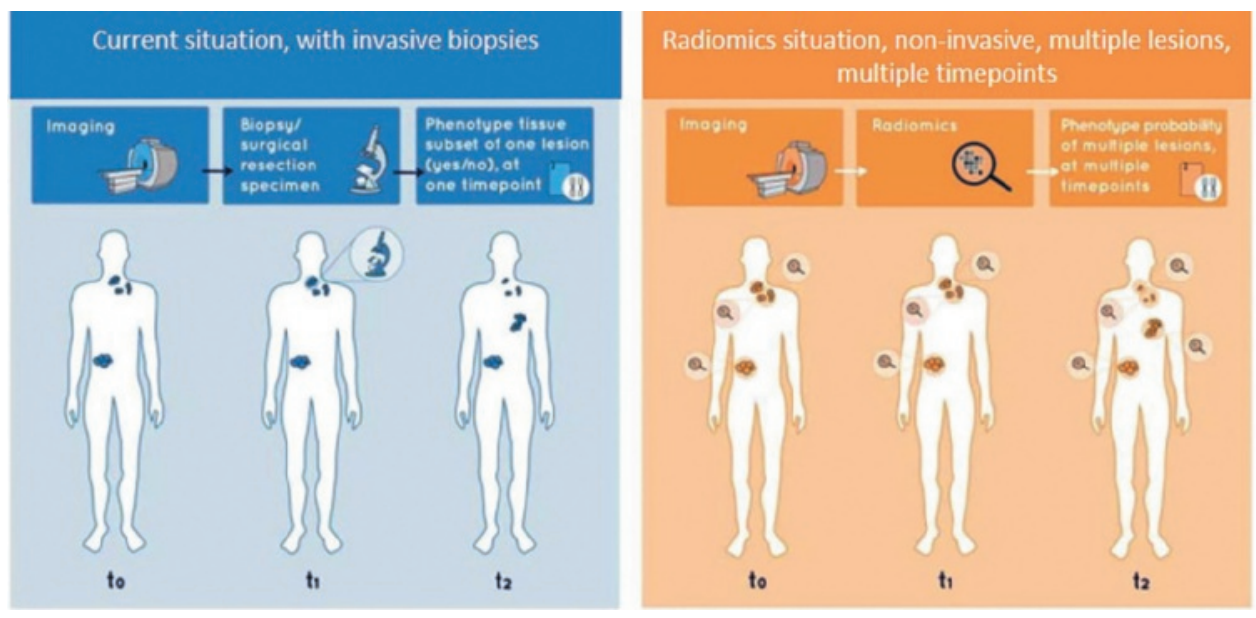

Figure 10.1: The left image shows the current situation, in which patients have to undergo invasive biopsies to determine the phenotype of tissue/tumors; the right image shows the future situation using radiomics. With radiomics the tumor phenotype potentially can be determined in a non-invasive way, with information from multiple lesions at multiple time points. Adjusted from Sanduleanu et al. [41].

$0.58, p=0.002)$ vs. 0.65 for the stage I-III group originally. Although, our cohort of patients was well characterized and in daily practice patients are viewed to have the same prognosis (stage IV, EGFR and ALK wildtype, non-squamous histology, WHO PS $<3$, chemotherapy eligible), by applying the signature and using a routine baseline CT scan we were able to select a group of patients with poor outcome [HR 1.45 (1.07-1.92), $p=0.02$ ]. In our univariate analysis next to the prognostic index of the radiomic signature [HR 1.46 (1.02-2.10), $\mathrm{p}=0.04]$, the known clinical prognostic factor WHO performance status [HR 1.52 (1.10-2.11)], N-stage [HR 1.84 (1.15-2.93)] and M-stage [HR 1.53 (1.07-2.19)] were also prognostic for overall survival. The 195 stage IV NSCLC patients were derived from two datasets. A reason for the lower c-index in the stage IV patients cohort, can be that the data from one of the cohorts $(n=137)$ was part of a multicenter study with no dedicated CT image protocol specified. There was quite some variation in the CT image acquisition and reconstruction parameters. Recent publications have shown that resampling of the voxel size leads to more reproducible CT features [47, 48]. This is a necessary step as Shafiq-Ul-Hassan et al. [47] showed that some radiomic features are voxel size dependent. To minimize the possible effect of the variation in image parameters, all scans were resampled, as recommended in Larue et al. [49], to a voxel size of $1 \times 1 \times 3$ $\mathrm{mm}^{3}$, which is comparable to the voxel size of the signature dataset. For radiomic studies it is important to use large datasets with standardized image acquisition and reconstruction protocols, to reduce noise. And to use resampling in validation studies when voxel sizes are not similar.

As the radiomic signature is based on analysis of the primary tumor, tumor volume might have impact on the outcome. In the stage I-III NSCLC signature cohort of Aerts et al. [46] the median tumor volume was $251 \mathrm{~cm}^{3}$ (2-489), while in our stage IV NSCLC cohort the median tumor volume was only $36 \mathrm{~cm}^{3},(0-769)$. In $33 \%$ of our cohort the tumors were staged as T4, which is comparable to the signature cohort, were $28 \%$ of 


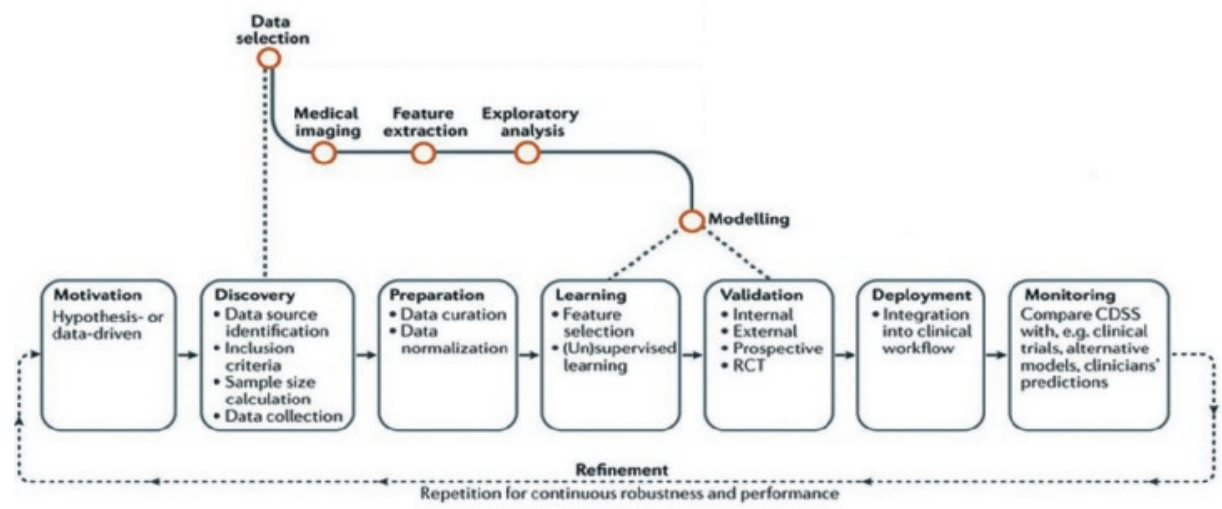

Figure 10.2: Process of radiomics, adjusted from Lambin et al. [42].

the tumors were staged as T4. A T4 staged tumor can be a large tumor, but it can as well mean that there are multiple nodules in the lung. In case of multiple nodules, the largest lesion was used for the radiomic analysis, which can also explain the difference in tumor volumes between the signature cohort and our cohort. Our cohort has an equal distribution of cT-stage compared to Aerts et al. [46], which confirms that cT-stage is not an indicator of tumor volume. In stage IV NSCLC, mainly the cM-stage determines the prognosis of a patient. With radiomics image characteristics of the primary tumor, but also of the nodes and metastases, image information can be easily used for prognostic models $[50,51,52]$. Although the radiomic signature developed in stage I-III NSCLC had as well prognostic value in stage IV NSCLC, the model is not reliable enough to use in clinical routine. While $\mathrm{N}$-stage and especially $\mathrm{M}$-stage are important prognostic factors in stage IV NSCLC, a radiomic signature including radiomic features extracted from lymph node metastases and distant metastases would potentially have a better prognostic value for overall survival.

Most radiomic studies use OS as endpoint, however it would also be interesting to have prognostic factors and models that predict distant metastases [53] or allow to predict the loss in quality of life. One of those prognostic factors that negatively affect survival in NSCLC is cachexia. Cachexia is a phenomenon of skeletal muscle and/or adipose tissue depletion in cancer patients, which is related to increased chemotherapy-induced toxicity, lower quality of life and lower survival rates in NSCLC patients $[54,55,56]$. Some studies have shown that the decrease in cross sectional area of the skeletal muscle is prognostic for overall survival $[54,55,56,57]$. Another study showed that a decreased volumetric muscle CT attenuation was associated with increased distant cancer recurrence [34]. In this thesis we showed that longitudinal differences in radiomic texture and intensity features are correlated to a decrease in cross sectional muscle volume, which could be a representation of myosteatosis. Next to developing models with survival as endpoint, prognostic models with for example cachexia as outcome could as well help to improve survival and quality 
of life in stage IV NSCLC patients.

\section{Methodological issues}

\section{PET imaging}

As mentioned before, to investigate, develop and validate the use of image biomarkers large datasets are needed. However, when using data from different hospitals or different PET/CT scanners a large variability in acquisition and reconstruction parameters can exist, which can influence the SUV. The European Association of Nuclear Medicine (EANM) provided in 2010 guidelines for a minimum standard for the acquisition and interpretation of ${ }^{18} \mathrm{~F}$-FDG PET/CT scans [58]. Earlier national image standardization guidelines, like the Netherlands protocol for standardization of quantitative whole-body FDG-PET/CT (NEDPAS) [59] and the German Guidelines for FDG-PET/CT in Oncology [60] have been integrated in the EANM guideline. Next to recommendations about patient preparation, image acquisition, image reconstruction and analysis also recommendations for quality control are given.

\section{Influence of PET acquisition time}

The NVALT12 trial, started in January 2011, study protocol prescribed that image acquisition had to be done according to the NEDPAS protocol, while image reconstruction could be done according to institutional standards. The NEDPAS protocol stated that emission scanning should start 55 to 65 minutes after the administration of FDG [59]. We showed that in the NVALT12 study, this was only the case in $34 \%$ of the scans. It is well known that lesion SUV for FDG continues to rise for at least 45 minutes after FDG injection, reaching a plateau between 60 and 90 minutes $[61,62,63]$. In the updated guidelines of the EANM version 2.0 from 2015 the acceptable uptake time window was changed to 55-75 minutes [64], which was the case for $59 \%$ of the scans in our study. Protocol deviations are quite common in clinical practice, a written survey among all Austrian PET/CT centers, showed that the average uptake time there was 55 minutes, with a range of 45 to 75 minutes, which is lower than in the EANM guidelines [65]. The optimal uptake time is as well tumor site specific, for liver imaging using ${ }^{18}$ F-FDG PET the optimal uptake time was shown to be 90 minutes after FDG injection [66]. A possible reason for deviations in our study is that the radiopharmaceutical start time, extracted from the Digital Imaging and Communications in Medicine (DICOM) header, was not the time of FDG administration, as we assumed. Because it is currently not possible to separately store information on injection time and calibration time in the DICOM header, some ambiguity about what time is in the DICOM header under the name radiopharmaceutical start time exists. Therefore, we recommend to take the acquisition time, near the midplane slice of the tumor, and note in the case report form (CRF) or DICOM header next to the injection time, the start time as well the calibration time. For response assessment it is important to have a minimum of variation in the uptake times of the repeated scans. In the uniform protocols for imaging in clinical trials (UPICT) guidelines it is stated that the acceptable variance in time interval is \pm 15 minutes, with a target of \pm 10 minutes [67]. In a consensus report for response assessment using PET is stated that uptake periods with less than 10 minutes differences do not lead to SUV variabilities [68]. In the NVALT 12 study only $19 \%$ of the scans 
fulfilled the UPICT criteria, which means that a variation in SUV was expected based on the differences in uptake times of the repeated scans.

For response assessment studies, when uptake times are both longer than 55 minutes and the difference between both uptake times is smaller than 10 minutes no differences in SUV values due to uptake time is expected. For shorter uptake times or larger differences it is recommended to consult a nuclear medicine expert before analyzing and interpreting the scans.

\section{Influence of PET reconstruction algorithms}

Reconstruction algorithms and voxel size are different for the various available PET systems and vendor dependent. Therefore, it is a challenge to ensure that images of PET scanners from different vendors are quantitatively comparable [69]. Point-spread-function (PSF) reconstructed images showed significantly higher SUV max and SUV mean values, as well as higher heterogeneity and a wider range of heterogeneity compared to ordered subset expectation maximization (OSEM) reconstructed images. Using PSF reconstruction with adjusting the Gaussian filtering to meet harmonizing standards resulted in similar SUV values [70]. $S U V_{\text {ref, }}$ applies a reconstruction-protocol specific filter to clinical PET scans to reduce reconstruction depended variation in SUV measurements, enabling increased confidence in quantitative comparisons between institutes [71]. Multiple studies have shown that liver metabolism can be used as reference organ due to its stable uptake over time and therefore, suitable for assessment of scan parameters [72]. In this thesis, a quality score was developed, in which for every parameter that was guideline compliant a point was given. The total number of points was divided by the number of known parameters. Parameters that were scored, are the uptake time, the reconstruction method, the convolution kernel used, the matrix size and the applied corrections (attenuation, scatter and randoms). The average quality score over all hospitals was $69 \%$. However, we could not show a relation between the quality score and $S U V_{\text {mean }}$ of the liver. Kuhnert et al. [73] showed that for parameters other than reconstruction method and settings (e.g. injected activity and uptake time), the $\mathrm{SUV}_{\text {mean }}$ of the liver could be used for quality assessment, in compliance with the EANM guideline, meaning the large variability in SUV $_{\text {mean }}$ of the liver is most probably caused by the differences in uptake time and net injected activity. Our results are in agreement with Hristova et al. [72], the variability in SUV $_{\text {mean }}$ of the liver is slightly smaller for the scans with a guideline compliant uptake time than for the scans with non-compliant uptake times. Therefore, for future multicenter trials using PET scans for response assessment it is recommended to do a prospective central quality-review during patient inclusion to improve compliance with image standardization.

Although many factors influence the SUV, a study investigating the stability of FDGPET radiomics features showed that $71 \%$ of the features were test-retest stable and $91 \%$ were inter-observer stable [74]. Another study showed that for PET radiomics the fixedbin-number discretization method resulted in more stable SUV values than the fixed-binsize discretization method [75]. For radiomics the segmentation process can have a large influence. It is shown that some texture features are highly correlated with the metabolic tumor volume. Only 17 of 31 investigated texture features were robust with respect to tumor segmentation method [76]. Other radiomic features, like skewness, cluster shade and zone percentage, are sensitive for differences in reconstruction settings [77].

When using ${ }^{18} \mathrm{~F}-\mathrm{FDG}$ PET scans from different institutes, performed on different PET scanners it is important to check if all institutes implemented the EANM guidelines. This can be checked by performing a prospective quality review of one scan per institute 
before patient inclusion. When different reconstruction algorithms are used, SUV ref can be used to minimize variation in SUV values. Before radiomics using ${ }^{18} \mathrm{~F}$-FDG PET can show it's potential, PET acquisition and reconstruction need to be standardized. For the radiomics process the PET scans need to be discretized using a fixed-bin-number method and segmentation need to be done using a standardized threshold.

\section{CE-CT imaging}

For stage III NSCLC patients, who are eligble for treatment with curative intent, current guidelines advise a post-contrast MRI or CE-CT of the brain to exclude brain metastases [78]. In (CE)-CT imaging guidelines [79, 80], no recommendations are made on the minimal technical requirements. However, while medical images are an integral part of oncological patient records and therefore reviewed by many different specialists, it is relevant that besides imaging experts, other clinicians are also aware that the diagnostic value of a scan is influenced by the applied imaging protocol. It is strongly recommended that the radiologist is informed about the indication for the requested scan and therefore, clinicians must provide all clinical information that is necessary to determine the optimal imaging protocol to apply. On the other side, for non-imaging clinicians it is important to evaluate whether the applied imaging protocol is indeed adequate for the requested purpose. The quality recommendations we developed potentially lead to a uniform clinical interpretation and could be helpful during multidisciplinary meetings. Although, the clinical impact should be tested in a prospective study.

In CE-CT guidelines for brain imaging, the importance of the patient position is not reported. The FOV of the head, should be adjusted to the size of the head, and the arms should be at thorax level to reduce artifacts (e.g. beam hardening). Furthermore, the sole fact that a scan was made with iodine contrast enhancement does not make the CT scan of the brain of proper diagnostic quality: timing of the scan relative to contrast injection and the amount of contrast material injected are important. In this thesis, the quality issues of CE-CT scans in a multicenter study were discussed.

\section{Influence acquisition parameters on $\mathrm{CT}$ radiomics}

We investigated the influence of different CT-scanners, slice thicknesses, exposures and gray-level discretization on radiomic feature values and their stability, with a dedicated phantom study containing several inserts. CT-scanner, slice thickness and bin width affected radiomic feature values, whereas no effect of exposure was observed. One of the scanners had a much wider range of Hounsfield units (HU) for the insert that had CT properties most similar to tumors, while the same radiation dose output and acquisition protocol was used as the other CT scanners, this might be caused by an incorrect calibration of the scanner. When using heterogeneous datasets, images from different scanners and/or different hospitals, for radiomics, it is therefore important to perform a robustness analysis (e.g. test-retest analysis or multiple delineation test) [81], to eliminate features that are not robust across different scanners or scan protocols, or apply corrections or post-processing steps.

We also showed that a large proportion of $\mathrm{CT}$ radiomic features is influenced by the slice thickness used for reconstruction, but the variability in feature values could be decreased by resampling. This is in line with Shafiq-ul Hassan et al. [47] who showed that resampling reduced the feature stability from $\%$ COV $>70 \%$ to $\%$ COV $>30 \%$. Therefore, we recommend to perform resampling prior to any radiomic analysis. Despite 
the fact that resampling can greatly improve the robustness of radiomic features [47], we advise to keep the voxel size as consistent as possible during image acquisition, because the variability in feature values is even lower when the voxel size is equal for all images included in a study. This should also be taken into account when comparing training and validation datasets that might be reconstructed into different voxel sizes. The distribution of radiomic feature values will be influenced by the different voxel sizes, even if resampling was applied. Therefore, care should be taken by using interpolation methods to change the resolution. Our results demonstrate that linear interpolation resulted in the narrowest feature value range for $48 \%$ of the features and cubic interpolation for $30 \%$ of the features, whereas nearest neighbor interpolation had the widest range for $61 \%$ of the features. Therefore, cubic or linear interpolation are preferred over nearest neighbor interpolation, when resampling to $1 \times 1 \times 3 \mathrm{~mm}^{3}$ voxels. For resampling it is important to look at the distribution of slice thicknesses, resampling to smaller slice thicknesses, lead to extrapolation of the data and therefore to less reliable results.

For none of the features, we could see a clear relationship between feature value and the exposure. A study investigating the influence of tube current on radiomic features showed that tube current had more effect on features extracted from homogeneous materials than from materials that are more similar to tissues. Their results indicate that variable $x$-ray tube current, in dose $(\mathrm{mA})$ modulated CT scans, are unlikely to have a large effect on radiomic features [82].

For CT radiomic studies it is important to use a standardized image acquisition and reconstruction protocol. Differences in slice thickness can be reduced by resampling the CT scans to a specific voxel size using a linear interpolation method. Although no difference in radiomic features was seen for different exposures, the influence of contrast agents on radiomic feature stability need to be investigated. And also the effect of different reconstruction kernels need to be looked into.

\section{Influence of pre-processing steps}

Almost all feature values change when choosing another bin width for gray-level discretization. For a subset of features, a very small or very large bin width resulted in very different feature values across scans, whereas feature values are more similar with a bin size in the order of $25 \mathrm{HU}$. Although the feature values change for different bin widths, we were not able to show that the stability of radiomic features is greatly influenced by the choice of bin width. Our finding is the contrary of what Shafiq-ul-Hassan et al. [47] described. They found that only seven out of 51 features were reproducible, independent of the gray-level discretization. Next to that, they found that seventeen out of 44 features showed a trend with varying number of gray levels, which could be a linear, quadratic or cubic type relation. Normalization of feature values by the number of gray levels reduced the variation in feature values. However, these results imply that the choice of bin width could alter the prognostic values of a certain radiomic signature. In contrast to $\mathrm{PET}$, for $\mathrm{CT}$, the stability of radiomic features is similar when discretizing using a fixed-bin-size or a fixed number of bins. It is strongly recommended to be consistent and always clearly report which pre-processing steps have been used, to improve the reproducibility and (external) validation of radiomic studies [42]. Next to that, it is important to visualize the distribution of HUs before setting the discretization bin size. This is especially important when new regions of interest are used, which could have a smaller HU range than tumors have. 


\section{MR imaging}

While CT and PET imaging are quantitative imaging modalities, MR is more qualitative (T1-weighted, T2-weighted). PET images uptake of radiotraces in tissues and is measured in SUV, in CT the absorption of $\mathrm{x}$-rays in tissues is measured and quantified in HUs which are related to electron densities. Qualitative $M R$ relies on the interpretation of tissue contrast that results from experimental pulse sequences. Quantitative MR measures tissue specific parameters independent of experimental design. An example of quantitative MR is diffusion weighted (DWI) MR [83]. In DWI MRI the random Brownian motion of water molecules within a voxel of tissue is measured and is quantified using the apparent diffusion coefficient (ADC). For MR imaging it is important to use the right combination of sequences for a complete overview. Next to anatomical information also information about diffusion and perfusion can be obtained which helps to distinguish benign lesions from malignant lesions. For a proper resolution, the slice thickness should be between 3-5 mm and a field strength of at least $1.5 \mathrm{~T}$ (for adequate signal-to-noise ratio and resolution) should be minimal used. In a multicenter study, described in this thesis, in which we evaluated the added value of MRI with respect to CE-CT, in asymptomatic brain metastases, we could show that quality issues already exist in the study preparation phase. One hospital could not participate because their MRI scanner could not meet the required technical standards and two other hospitals had to adjust their MR protocol.

In a phantom study performed by Yang et al. [84], the dependence of image texture features on MR acquisition and reconstruction parameters was evaluated. They found that feature variance due to reconstruction algorithm and acceleration factor were generally smaller than feature differences between high- and low-grade tumors on T1- and T2weighted brain images [84]. Another study including MR scans from three institutes showed that a prognostic model trained on one institute could be validated in the data from the other institutes, which showed that a vendor independent model can be developed [85]. However, in prognostic or predictive model development large datasets are needed to train models and to validate models [86]. A study of Collins et al. [39] showed that to reliable validate a prognostic model a minimum of 100 events and ideally, 200 or more events are required. Using such large datasets mostly means that data have to be derived from different hospitals. A standardized quality assurance for DWI can harmonize DWI values in different institutes, which will potentially lead to more robust models [87]. To perform radiomics on MR data more research need to be done to compare MR images of different institutes. A phantom study help to identify which MR acquisition and reconstruction parameters influence stability of radiomic features.

\section{Future perspectives}

In 2017 the radiomics quality score (RQS) was published [42]. The RQS allows to aid in the quality assessment of past and future radiomic studies. It can help clinicians and researchers to easily ascertain, whether a radiomic study is compliant with best practice procedures or alternatively, whether the study investigators have sufficiently justified any non-compliance with guidelines. The RQS scores the used image protocol, the performed segmentation, the feature selection process, the model development, the biological correlation, the statistics, the comparison to the gold standard and the transparency of the analysis. The use of this quality score may in the future lead to higher quality radiomic studies, while more and more points from the RQS are incorporated. In our review about tracking tumor 
biology with radiomics, the RQS was used to score all included articles [41]. While the RQS score mentions the use of a biological correlate, it does not quantify the link between tumor biology and radiomics. We found that only a minor discrepancy between lower RQS studies and the claims about clinical significance existed. We also showed that there are some inter-rater interpretation differences of the different RQS score components. The RQS score should be made more robust and easily interpretable in the future and will then potentially lead to more standardized radiomic workflows.

To create robust prognostic models representing the complete population, larger datasets are needed than currently used in almost all analyses. Even when image acquisition and reconstruction are standardized, it is difficult to analyze all data in a central location, due to ethical and legal boundaries prohibiting data sharing. Using distributed learning, it is possible to share data, without identifiable patient data leaving the hospitals. This can be done by instead of storing the data in a central hospital, send the model to all participating hospitals. A proof-of-concept study of Deist et al. [88], learned exemplary support vector machine models, to predict dyspnea. They found that the training AUC over all centers was 0.63 and varied from 0.60 to 0.64 in a leave-one-out cross validation. With this proof-of-concept study, it is shown that multi-centric rapid learning healthcare is feasible and that in the future, models can be developed using distributed databases, across national borders, accompanying technical, legal and ethical issues.

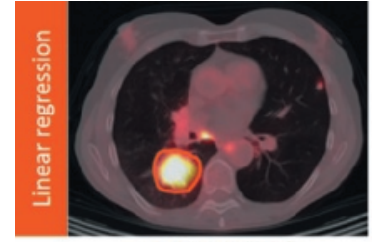

Delineated CT

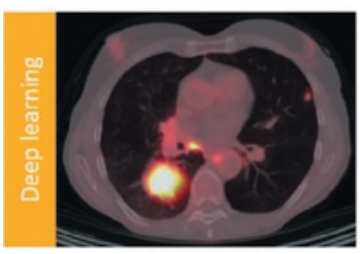

Undelineated Cl

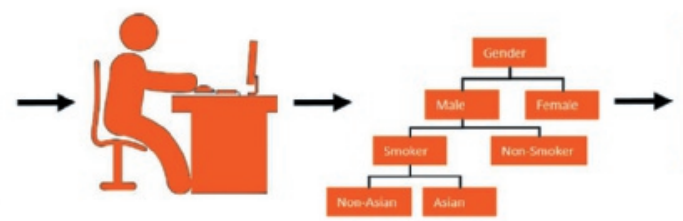

Classification

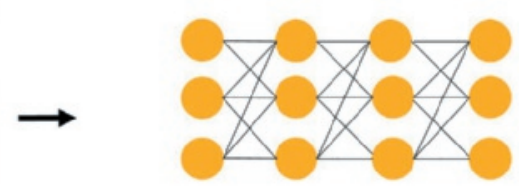

Feature extraction + Classification
EGFR-mutated

Hypoxic

Calcifications

Heterogeneous

Output

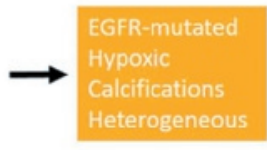

Output

Figure 10.3: The difference between machine learning and deep learning is the feature extraction and segmentation of the region of interest, reproduced from xenonstack.com.

When using distributed learning, large datasets will be available and to analyze them automated methods need to be used. In radiomics, the segmentation of the region of interest is the most time consuming step. In a study of Velazquez et al. [89], a semi-automatic CT-based segmentation method was compared to manual delineations of five physicians. They found that automatic segmented volumes, demonstrated a high inter-observer agreement, lower volume variability, smaller uncertainty areas and a strong correlation to surgically defined tumor volumes. Machine learning, a method in which statistics are used to give computers the ability to learn from data, can be used in medicine as well. Machine learning can be divided in machine learning with pre-processed 
feature input values and machine learning without preprocessing on the raw (image) input, the latter also called deep learning [90]. Deep learning does not require feature calculation or lesion segmentation, and it can be classified as end-to-end machine learning, that does the entire process from input images to final classification, see Figure 10.3. With the use of deep learning large datasets can be analyzed automatically and prognostic models will be more robust and population based. Nevertheless, to make reliable models with deep learning, large datasets are needed. Challenges in big data include data storage, information privacy and computer power.

To conclude, using distributed learning infrastructure with standardized data acquisition and reconstruction, deep learning in combination with radiomics and all other available prognostic information will lead to robust prognostic and predictive features in lung cancer. Prognostic and predictive models might be helpful in treatment decisions and may lead to decrease complications and increase quality of life. 


\section{References}

[1] P. H. Madsen et al. "Clinical utility of F-18 FDG PET-CT in the initial evaluation of lung cancer". In: Eur J Nucl Med Mol Imaging 43.11 (2016). European journal of nuclear medicine and molecular imaging, pp. 2084-97. DOI: 10.1007/s00259-0163407-4.

[2] P. Heusch et al. "Thoracic staging in lung cancer: prospective comparison of 18F-FDG PET/MR imaging and 18F-FDG PET/CT". In: J Nucl Med 55.3 (2014). Journal of nuclear medicine : official publication, Society of Nuclear Medicine, pp. 373-8. DOI: $10.2967 /$ jnumed.113.129825.

[3] E. A. Eisenhauer et al. "New response evaluation criteria in solid tumours: revised RECIST guideline (version 1.1)". In: Eur J Cancer 45.2 (2009). European journal of cancer (Oxford, England : 1990), pp. 228-47. DOI: 10.1016/j.ejca.2008.10.026.

[4] World Health Organization. WHO handbook for reporting results of cancer treatment. Geneva: World Health Organization, 1979.

[5] G. L. Rosner, W. Stadler, and M. J. Ratain. "Randomized discontinuation design: application to cytostatic antineoplastic agents". In: J Clin Oncol 20.22 (2002). Journal of clinical oncology : official journal of the American Society of Clinical Oncology, pp. 4478-84. DOI: 10.1200/jco.2002.11.126.

[6] L. C. Michaelis and M. J. Ratain. "Measuring response in a post-RECIST world: from black and white to shades of grey". In: Nat Rev Cancer 6.5 (2006). Nature reviews. Cancer, pp. 409-14. DOI: 10.1038/nrc1883.

[7] K. Skougaard et al. "Comparison of EORTC criteria and PERCIST for PET/CT response evaluation of patients with metastatic colorectal cancer treated with irinotecan and cetuximab". In: J Nucl Med 54.7 (2013). Journal of nuclear medicine : official publication, Society of Nuclear Medicine, pp. 1026-31. DOI: 10.2967/jnumed.112.111757.

[8] R. L. Wahl et al. "From RECIST to PERCIST: Evolving Considerations for PET response criteria in solid tumors". In: J Nucl Med 50 Suppl 1 (2009). Journal of nuclear medicine : official publication, Society of Nuclear Medicine, 122S-50S. DOI: 10.2967 / jnumed. 108.057307. URL: http: / / www . ncbi .nlm. nih.gov/pubmed/ 19403881.

[9] M. P. Mac Manus et al. "Positron emission tomography is superior to computed tomography scanning for response-assessment after radical radiotherapy or chemoradiotherapy in patients with non-small-cell lung cancer". In: J Clin Oncol 21.7 (2003). Journal of clinical oncology : official journal of the American Society of Clinical Oncology, pp. 1285-92. DOI: 10.1200/jco.2003.07.054.

[10] M. Meignan et al. "Report on the 4th International Workshop on Positron Emission Tomography in Lymphoma held in Menton, France, 3-5 October 2012". In: Leuk Lymphoma 55.1 (2014). Leukemia \& lymphoma, pp. 31-7. DOI: 10.3109/10428194. 2013.802784.

[11] G. A. Turgeon et al. "What FDG-PET response-assessment method best predicts survival after curative-intent chemoradiation in non-small cell lung cancer (NSCLC): EORTC, PERCIST, Peter Mac or Deauville criteria?" In: J Nucl Med (2018). Journal of nuclear medicine : official publication, Society of Nuclear Medicine. DOI: 10.2967/ jnumed.118.214148. 
[12] E. Depardon et al. "FDG PET/CT for prognostic stratification of patients with metastatic breast cancer treated with first line systemic therapy: Comparison of EORTC criteria and PERCIST". In: PLoS One 13.7 (2018). PloS one, e0199529. DOI: $10.1371 /$ journal. pone.0199529.

[13] S. Odawara et al. "Tumor response to neoadjuvant chemotherapy in patients with esophageal cancer assessed with CT and FDG-PET/CT - RECIST 1.1 vs. PERCIST 1.0". In: Eur J Radiol 101 (2018). European journal of radiology, pp. 65-71. DOI: 10.1016/j.ejrad.2018.02.009.

[14] A. M. Dingemans et al. "A randomized phase II study comparing paclitaxel-carboplatin-bevacizumab with or without nitroglycerin patches in patients with stage IV nonsquamous nonsmall-cell lung cancer: NVALT12 (NCT01171170)". In: Ann Oncol 26.11 (2015). Annals of oncology : official journal of the European Society for Medical Oncology, pp. 2286-93. DOI: $10.1093 /$ annonc/mdv370.

[15] A. M. Dingemans et al. "First-line erlotinib and bevacizumab in patients with locally advanced and/or metastatic non-small-cell lung cancer: a phase II study including molecular imaging". In: Ann Oncol 22.3 (2011). Annals of oncology : official journal of the European Society for Medical Oncology / ESMO, pp. 559-66. DOI: 10.1093/ annonc/mdq391. URL: http://www.ncbi.nlm.nih.gov/pubmed/20702788.

[16] K. V. Ballman. "Biomarker: Predictive or Prognostic?" In: J Clin Oncol 33.33 (2015). Journal of clinical oncology : official journal of the American Society of Clinical Oncology, pp. 3968-71. DOI: 10.1200/jco.2015.63.3651.

[17] Biomarkers Definitions Working Group. "Biomarkers and surrogate endpoints: preferred definitions and conceptual framework." eng. In: Clinical pharmacology and therapeutics 69 (3 2001), pp. 89-95.

[18] D. Zhu et al. "Prognostic value of the maximum standardized uptake value of pretreatment primary lesions in small-cell lung cancer on 18F-FDG PET/CT: a metaanalysis". In: Acta Radiol (2017). Acta radiologica (Stockholm, Sweden : 1987), p. 284185117745907 . DOI: $10.1177 / 0284185117745907$.

[19] M. R. Kocher et al. "Pretreatment 18F-Fluorodeoxyglucose Positron Emission Tomography Standardized Uptake Values and Tumor Size in Medically Inoperable Nonsmall Cell Lung Cancer Is Prognostic of Overall 2-Year Survival After Stereotactic Body Radiation Therapy". In: J Comput Assist Tomogr 42.1 (2018). Journal of computer assisted tomography, pp. 146-150. DOI: $10.1097 /$ rct.0000000000000653.

[20] A. L. Chin et al. "Prognostic Value of Pretreatment FDG-PET Parameters in Highdose Image-guided Radiotherapy for Oligometastatic Non-Small-cell Lung Cancer". In: Clin Lung Cancer (2018). Clinical lung cancer. DOI: 10.1016/j.cllc.2018.04.003.

[21] E. J. Han et al. "Prognostic value of early response assessment using 18F-FDG $\mathrm{PET} / \mathrm{CT}$ in chemotherapy-treated patients with non-small-cell lung cancer". In: Nucl Med Commun 36.12 (2015). Nuclear medicine communications, pp. 1187-94. DOI: $10.1097 / \mathrm{mnm} .0000000000000382$.

[22] H. Wang et al. "Semiquantitative Computed Tomography Characteristics for Lung Adenocarcinoma and Their Association With Lung Cancer Survival". In: Clin Lung Cancer 16.6 (2015). Clinical lung cancer, e141-63. DOI: 10.1016/j.cllc.2015.05.007. 
[23] S. Yossi et al. "Early assessment of metabolic response by $18 \mathrm{~F}-\mathrm{FDG}$ PET during concomitant radiochemotherapy of non-small cell lung carcinoma is associated with survival: a retrospective single-center study". In: Clin Nucl Med 40.4 (2015). Clinical nuclear medicine, e215-21. DOI: 10.1097/rlu.0000000000000615.

[24] L. F. de Geus-Oei et al. "Chemotherapy response evaluation with 18F-FDG PET in patients with non-small cell lung cancer". In: J Nucl Med 48.10 (2007). Journal of nuclear medicine : official publication, Society of Nuclear Medicine, pp. 1592-8. DOI: 10.2967/jnumed.107.043414. URL: http://www.ncbi.nlm.nih.gov/pubmed/ 17873138.

[25] M. Vaidya et al. "Combined PET/CT image characteristics for radiotherapy tumor response in lung cancer". In: Radiother Oncol 102.2 (2012). Radiotherapy and oncology : journal of the European Society for Therapeutic Radiology and Oncology, pp. 239-45. DOI: 10.1016/j.radonc.2011.10.014.

[26] W. van Elmpt et al. "Multiparametric imaging of patient and tumour heterogeneity in non-small-cell lung cancer: quantification of tumour hypoxia, metabolism and perfusion". In: Eur J Nucl Med Mol Imaging 43.2 (2016). European journal of nuclear medicine and molecular imaging, pp. 240-8. DOI: 10.1007/s00259-015-3169-4.

[27] P. F. Rijken et al. "Spatial relationship between hypoxia and the (perfused) vascular network in a human glioma xenograft: a quantitative multi-parameter analysis". In: Int J Radiat Oncol Biol Phys 48.2 (2000). International journal of radiation oncology, biology, physics, pp. 571-82.

[28] H. Yasuda et al. "Randomized phase II trial comparing nitroglycerin plus vinorelbine and cisplatin with vinorelbine and cisplatin alone in previously untreated stage IIIB/IV non-small-cell lung cancer". In: J Clin Oncol 24.4 (2006). Journal of clinical oncology : official journal of the American Society of Clinical Oncology, pp. 688-94. DOI: 10.1200/jco.2005.04.0436.

[29] N. E. Matthews et al. "Nitric oxide-mediated regulation of chemosensitivity in cancer cells". In: J Natl Cancer Inst 93.24 (2001). Journal of the National Cancer Institute, pp. $1879-85$.

[30] C. Nahmias et al. "Time course of early response to chemotherapy in non-small cell lung cancer patients with 18F-FDG PET/CT". In: J Nucl Med 48.5 (2007). Journal of nuclear medicine : official publication, Society of Nuclear Medicine, pp. 744-51. DOI: $10.2967 /$ jnumed.106.038513.

[31] C. M. Zegers et al. "In vivo quantification of hypoxic and metabolic status of NSCLC tumors using [18F]HX4 and [18F]FDG-PET/CT imaging". In: Clin Cancer Res 20.24 (2014). Clinical cancer research : an official journal of the American Association for Cancer Research, pp. 6389-97. DOI: 10.1158/1078-0432.ccr-14-1524.

[32] A. A. van der Veldt, E. F. Smit, and A. A. Lammertsma. "Positron Emission Tomography as a Method for Measuring Drug Delivery to Tumors in vivo: The Example of $[(11) C]$ docetaxel". In: Front Oncol 3 (2013). Frontiers in oncology, p. 208. DOI: $10.3389 /$ fonc.2013.00208.

[33] E. Laffon et al. "Variability of average SUV from several hottest voxels is lower than that of SUVmax and SUVpeak". In: Eur Radiol 24.8 (2014). European radiology, pp. 1964-70. DOI: $10.1007 /$ s00330-014-3222-x. 
[34] Q. Li et al. "Imaging features from pretreatment CT scans are associated with clinical outcomes in nonsmall-cell lung cancer patients treated with stereotactic body radiotherapy". In: Med Phys 44.8 (2017). Medical physics, pp. 4341-4349. DOI: $10.1002 / \mathrm{mp} .12309$.

[35] B. Foster et al. "Skeletal Muscle Metrics on Clinical (18)F-FDG PET/CT Predict Health Outcomes in Patients with Sarcoma". In: J Nat Sci 4.5 (2018). Journal of nature and science.

[36] E. Segal et al. "Decoding global gene expression programs in liver cancer by noninvasive imaging". In: Nat Biotechnol 25.6 (2007). Nature biotechnology, pp. 675-80. DOI: $10.1038 /$ nbt1306.

[37] S. Rizzo et al. "CT Radiogenomic Characterization of EGFR, K-RAS, and ALK Mutations in Non-Small Cell Lung Cancer". In: Eur Radiol 26.1 (2016). European radiology, pp. 32-42. DOI: 10.1007/s00330-015-3814-0.

[38] S. S. F. Yip et al. "Associations between radiologist-defined semantic and automatically computed radiomic features in non-small cell lung cancer". In: Sci Rep 7.1 (2017). Scientific reports, p. 3519. DOI: 10.1038/s41598-017-02425-5.

[39] G. S. Collins, E. O. Ogundimu, and D. G. Altman. "Sample size considerations for the external validation of a multivariable prognostic model: a resampling study". In: Stat Med 35.2 (2016). Statistics in medicine, pp. 214-26. DOI: 10.1002/sim.6787.

[40] S. S. Yip and H. J. Aerts. "Applications and limitations of radiomics". In: Phys Med Biol 61.13 (2016). Physics in medicine and biology, R150-66. DOI: 10.1088/0031$9155 / 61 / 13 / r 150$.

[41] S. Sanduleanu et al. "Tracking tumor biology with radiomics: A systematic review utilizing a radiomics quality score". In: Radiother Oncol 127.3 (2018). Radiotherapy and oncology : journal of the European Society for Therapeutic Radiology and Oncology, pp. 349-360. DOI: 10.1016/j.radonc.2018.03.033.

[42] P. Lambin et al. "Radiomics: the bridge between medical imaging and personalized medicine". In: Nat Rev Clin Oncol 14.12 (2017). Nature reviews. Clinical oncology, pp. 749-762. DOI: 10.1038/nrclinonc.2017.141.

[43] B. M. Virginia et al. "Prognostic value of histogram analysis in advanced nonsmall cell lung cancer: a radiomic study". In: Oncotarget 9.2 (2018). Oncotarget, pp. 1906-1914. DOI: 10.18632/oncotarget. 22316.

[44] L. Zhang et al. "Quantitative Biomarkers for Prediction of Epidermal Growth Factor Receptor Mutation in Non-Small Cell Lung Cancer". In: Transl Oncol 11.1 (2018). Translational oncology, pp. 94-101. DOI: 10.1016/j.tranon.2017.10.012.

[45] T. P. Coroller et al. "Radiomic phenotype features predict pathological response in non-small cell lung cancer". In: Radiother Oncol 119.3 (2016). Radiotherapy and oncology : journal of the European Society for Therapeutic Radiology and Oncology, pp. 480-6. DOI: 10.1016/j.radonc.2016.04.004.

[46] H. J. Aerts et al. "Decoding tumour phenotype by noninvasive imaging using a quantitative radiomics approach". In: Nat Commun 5 (2014). Nature communications, p. 4006. DOI: 10.1038/ncomms5006.

[47] M. Shafiq-Ul-Hassan et al. "Intrinsic dependencies of CT radiomic features on voxel size and number of gray levels". In: Med Phys 44.3 (2017). Medical physics, pp. 1050-1062. DOI: 10.1002/mp.12123. 
[48] D. Mackin et al. "Harmonizing the pixel size in retrospective computed tomography radiomics studies". In: PLoS One 12.9 (2017). PloS one, e0178524. DOI: 10.1371/ journal. pone.0178524.

[49] R. T. H. M. Larue et al. "Influence of gray level discretization on radiomic feature stability for different CT scanners, tube currents and slice thicknesses: a comprehensive phantom study". In: Acta Oncol In press (2017), p. 11. DOI: 10.1080/0284186X.2017.1351624

[50] L. D. Chen et al. "Multiparametric radiomics improve prediction of lymph node metastasis of rectal cancer compared with conventional radiomics". In: Life Sci (2018). Life sciences. DOI: 10.1016/j.Ifs.2018.07.007.

[51] T. P. Coroller et al. "Radiomic-Based Pathological Response Prediction from Primary Tumors and Lymph Nodes in NSCLC". In: J Thorac Oncol 12.3 (2017). Journal of thoracic oncology : official publication of the International Association for the Study of Lung Cancer, pp. 467-476. DOI: 10.1016/j.jtho.2016.11.2226.

[52] S. Carvalho et al. "Early variation of FDG-PET radiomics features in NSCLC is related to overall survival the delta radiomics concept". In: Radiotherapy and Oncology 118 (Sept. 2018), S20-S21. ISSN: 0167-8140. DOI: 10 . 1016 / s0167 - 8140(16 ) $30042-1 . \quad$ URL: https://doi.org/10.1016/S0167-8140(16)30042-1.

[53] T. P. Coroller et al. "CT-based radiomic signature predicts distant metastasis in lung adenocarcinoma". In: Radiother Oncol 114.3 (2015). Radiotherapy and oncology : journal of the European Society for Therapeutic Radiology and Oncology, pp. 345-50. DOI: 10.1016/j.radonc.2015.02.015.

[54] B. Sjoblom et al. "Low muscle mass is associated with chemotherapy-induced haematological toxicity in advanced non-small cell lung cancer". In: Lung Cancer 90.1 (2015). Lung cancer (Amsterdam, Netherlands), pp. 85-91. DOI: 10.1016/j.lungcan.2015.07.001.

[55] A. Bye et al. "Muscle mass and association to quality of life in non-small cell lung cancer patients". In: J Cachexia Sarcopenia Muscle 8.5 (2017). Journal of cachexia, sarcopenia and muscle, pp. 759-767. DOI: 10.1002/jcsm.12206.

[56] L. Martin et al. "Cancer cachexia in the age of obesity: skeletal muscle depletion is a powerful prognostic factor, independent of body mass index". In: J Clin Oncol 31.12 (2013). Journal of clinical oncology : official journal of the American Society of Clinical Oncology, pp. 1539-47. DOI: 10.1200/jco.2012.45.2722.

[57] B. Sjoblom et al. "Skeletal muscle radiodensity is prognostic for survival in patients with advanced non-small cell lung cancer". In: Clin Nutr 35.6 (2016). Clinical nutrition (Edinburgh, Scotland), pp. 1386-1393. DOI: 10.1016/j.clnu.2016.03.010.

[58] R. Boellaard et al. "FDG PET and PET/CT: EANM procedure guidelines for tumour PET imaging: version 1.0". In: Eur J Nucl Med Mol Imaging 37.1 (2010). European journal of nuclear medicine and molecular imaging, pp. 181-200. DOI: 10.1007/ s00259-009-1297-4.

[59] R. Boellaard et al. "The Netherlands protocol for standardisation and quantification of FDG whole body PET studies in multi-centre trials". In: Eur J Nucl Med Mol Imaging 35.12 (2008). European journal of nuclear medicine and molecular imaging, pp. 2320-33. DOI: 10.1007/s00259-008-0874-2. URL: http://www.ncbi.nlm.nih. gov/pubmed/18704407. 
[60] B. J. Krause et al. "[FDG-PET/CT in oncology. German Guideline]". In: Nuklearmedizin 46.6 (2007). Nuklearmedizin. Nuclear medicine, pp. 291-301.

[61] S. Beaulieu et al. "SUV varies with time after injection in (18)F-FDG PET of breast cancer: characterization and method to adjust for time differences". In: J Nucl Med 44.7 (2003). Journal of nuclear medicine : official publication, Society of Nuclear Medicine, pp. 1044-50.

[62] D. S. Binns et al. "Compliance with PET acquisition protocols for therapeutic monitoring of erlotinib therapy in an international trial for patients with non-small cell lung cancer". In: Eur J Nucl Med Mol Imaging 38.4 (2011). European journal of nuclear medicine and molecular imaging, pp. 642-50. DOI: 10.1007/s00259-010-1665-0.

[63] P. E. Kinahan and J. W. Fletcher. "Positron emission tomography-computed tomography standardized uptake values in clinical practice and assessing response to therapy". In: Semin Ultrasound CT MR 31.6 (2010). Seminars in ultrasound, CT, and MR, pp. 496-505. DOI: 10.1053/j.sult.2010.10.001.

[64] R. Boellaard et al. "FDG PET/CT: EANM procedure guidelines for tumour imaging: version 2.0". In: Eur J Nucl Med Mol Imaging 42.2 (2015). European journal of nuclear medicine and molecular imaging, pp. 328-54. DOI: $10.1007 /$ s00259-0142961-x. URL: http://www.ncbi.nlm.nih.gov/pubmed/25452219.

[65] I. Rausch et al. "Variation of system performance, quality control standards and adherence to international FDG-PET/CT imaging guidelines. A national survey of PET/CT operations in Austria". In: Nuklearmedizin 53.6 (2014). Nuklearmedizin. Nuclear medicine, pp. 242-8. DOI: 10.3413/Nukmed-0665-14-05.

[66] Z. Al-Faham et al. "Optimizing 18F-FDG Uptake Time Before Imaging Improves the Accuracy of PET/CT in Liver Lesions". In: J Nucl Med Technol 44.2 (2016). Journal of nuclear medicine technology, pp. 70-2. DOI: 10.2967/jnmt.115.169953.

[67] M. M. Graham et al. "Summary of the UPICT Protocol for 18F-FDG PET/CT Imaging in Oncology Clinical Trials". In: J Nucl Med 56.6 (2015). Journal of nuclear medicine : official publication, Society of Nuclear Medicine, pp. 955-61. DOI: 10. 2967 /jnumed.115.158402.

[68] L. K. Shankar et al. "Consensus recommendations for the use of 18F-FDG PET as an indicator of therapeutic response in patients in National Cancer Institute Trials". In: J Nucl Med 47.6 (2006). Journal of nuclear medicine : official publication, Society of Nuclear Medicine, pp. 1059-66.

[69] M. M. Graham, R. D. Badawi, and R. L. Wahl. "Variations in PET/CT methodology for oncologic imaging at U.S. academic medical centers: an imaging response assessment team survey". In: J Nucl Med 52.2 (2011). Journal of nuclear medicine : official publication, Society of Nuclear Medicine, pp. 311-7. DOI: 10.2967/jnumed.109.074104.

[70] C. Lasnon et al. "(18)F-FDG PET/CT heterogeneity quantification through textural features in the era of harmonisation programs: a focus on lung cancer". In: Eur $J$ Nucl Med Mol Imaging 43.13 (2016). European journal of nuclear medicine and molecular imaging, pp. 2324-2335. DOI: 10.1007/s00259-016-3441-2.

[71] M. D. Kelly and J. M. Declerck. "SUVref: reducing reconstruction-dependent variation in PET SUV". In: EJNMMI Res 1.1 (2011). EJNMMI research, p. 16. DOI: $10.1186 / 2191-219 x-1-16$. 
[72] I. Hristova et al. "Retrospective quality control review of FDG scans in the imaging sub-study of PALETTE EORTC 62072/VEG110727: a randomized, double-blind, placebo-controlled phase III trial". In: Eur J Nucl Med Mol Imaging 42.6 (2015). European journal of nuclear medicine and molecular imaging, pp. 848-57. DOI: 10. 1007/s00259-015-3002-0.

[73] G. Kuhnert et al. "Impact of PET/CT image reconstruction methods and liver uptake normalization strategies on quantitative image analysis". In: Eur J Nucl Med Mol Imaging 43.2 (2016). European journal of nuclear medicine and molecular imaging, pp. 249-58. DOI: $10.1007 / \mathrm{s} 00259-015-3165-8$.

[74] R. T. Leijenaar et al. "Stability of FDG-PET Radiomics features: an integrated analysis of test-retest and inter-observer variability". In: Acta Oncol 52.7 (2013). Acta oncologica (Stockholm, Sweden), pp. 1391-7. DOI: 10.3109/0284186x.2013. 812798 .

[75] L. Presotto et al. "PET textural features stability and pattern discrimination power for radiomics analysis: An "ad-hoc" phantoms study". In: Phys Med 50 (2018). Physica medica : PM : an international journal devoted to the applications of physics to medicine and biology : official journal of the Italian Association of Biomedical Physics (AIFB), pp. 66-74. DOI: 10.1016/j.ejmp.2018.05.024.

[76] F. Orlhac et al. "Tumor texture analysis in 18F-FDG PET: relationships between texture parameters, histogram indices, standardized uptake values, metabolic volumes, and total lesion glycolysis". In: J Nucl Med 55.3 (2014). Journal of nuclear medicine : official publication, Society of Nuclear Medicine, pp. 414-22. DOI: 10.2967/jnumed.113.129858.

[77] J. Yan et al. "Impact of Image Reconstruction Settings on Texture Features in 18FFDG PET". In: J Nucl Med 56.11 (2015). Journal of nuclear medicine : official publication, Society of Nuclear Medicine, pp. 1667-73. DOI: 10.2967/jnumed.115. 156927.

[78] L. E. Hendriks et al. "Screening for brain metastases in patients with stage III non-small cell lung cancer: Is there additive value of magnetic resonance imaging above a contrast-enhanced computed tomography of the brain?" In: Lung Cancer 80.3 (2013). Lung cancer (Amsterdam, Netherlands), pp. 293-7. DOI: 10.1016/j. lungcan.2013.02.006.

[79] P. E. Postmus et al. "Early and locally advanced non-small-cell lung cancer (NSCLC): ESMO Clinical Practice Guidelines for diagnosis, treatment and follow-up". In: Ann Oncol 28 (2017). Annals of oncology : official journal of the European Society for Medical Oncology, pp. iv1-iv21. DOI: 10.1093/annonc/mdx222.

[80] D. S. Ettinger et al. "Non-Small Cell Lung Cancer, Version 5.2017, NCCN Clinical Practice Guidelines in Oncology". In: J Natl Compr Canc Netw 15.4 (2017). Journal of the National Comprehensive Cancer Network: JNCCN, pp. 504-535.

[81] J. E. van Timmeren et al. "Survival prediction of non-small cell lung cancer patients using radiomics analyses of cone-beam CT images". In: Radiother Oncol 123.3 (2017). Radiotherapy and oncology : journal of the European Society for Therapeutic Radiology and Oncology, pp. 363-369. DOI: 10.1016/j.radonc.2017.04.016. 
[82] D. Mackin et al. "Effect of tube current on computed tomography radiomic features". In: Sci Rep 8.1 (2018). Scientific reports, p. 2354. DOI: 10.1038/s41598-018-20713-6.

[83] A. F. Mills et al. "Principles of Quantitative MR Imaging with Illustrated Review of Applicable Modular Pulse Diagrams". In: Radiographics 37.7 (2017). Radiographics : a review publication of the Radiological Society of North America, Inc, pp. 2083-2105. DOI: 10.1148/rg.2017160099.

[84] F. Yang et al. "Evaluation of radiomic texture feature error due to MRI acquisition and reconstruction: A simulation study utilizing ground truth". In: Phys Med 50 (2018). Physica medica : PM : an international journal devoted to the applications of physics to medicine and biology : official journal of the Italian Association of Biomedical Physics (AIFB), pp. 26-36. DOI: 10.1016/j.ejmp.2018.05.017.

[85] N. Dinapoli et al. "Magnetic Resonance, Vendor-independent, Intensity Histogram Analysis Predicting Pathologic Complete Response After Radiochemotherapy of Rectal Cancer". In: Int J Radiat Oncol Biol Phys (2018). International journal of radiation oncology, biology, physics. DOI: 10.1016/j.ijrobp.2018.04.065.

[86] P. Royston and D. G. Altman. "External validation of a Cox prognostic model: principles and methods". In: BMC Med Res Methodol 13 (2013). BMC medical research methodology, p. 33. DOI: 10.1186/1471-2288-13-33.

[87] G. Belli et al. "Quality assurance multicenter comparison of different MR scanners for quantitative diffusion-weighted imaging". In: J Magn Reson Imaging 43.1 (2016). Journal of magnetic resonance imaging : JMRI, pp. 213-9. DOI: 10.1002/jmri.24956.

[88] T. M. Deist et al. "Infrastructure and distributed learning methodology for privacypreserving multi-centric rapid learning health care: euroCAT". In: Clin Transl Radiat Oncol 4 (2017). Clinical and translational radiation oncology, pp. 24-31. DOI: 10. 1016/j.ctro.2016.12.004.

[89] E. Rios Velazquez et al. "A semiautomatic CT-based ensemble segmentation of lung tumors: comparison with oncologists' delineations and with the surgical specimen". In: Radiother Oncol 105.2 (2012). Radiotherapy and oncology : journal of the European Society for Therapeutic Radiology and Oncology, pp. 167-73. DOI: $10.1016 / \mathrm{j}$.radonc.2012.09.023.

[90] K. Suzuki. "Overview of deep learning in medical imaging". In: Radiol Phys Technol 10.3 (2017). Radiological physics and technology, pp. 257-273. DOI: 10.1007 / s12194-017-0406-5. 

Appendices 

Summary 


\section{Background and aim of the thesis}

In the Netherlands, each year more than 100,000 people are diagnosed with cancer, and although many of them are cured, still about 40,000 patients yearly die. Of those 40,000 yearly cancer deaths, $24 \%$ is due to lung cancer. The overall survival rate in lung cancer depends on the stage of the disease. Patients with distant metastases are classified as stage IV, independent of the size of the tumor and the number of involved lymph nodes. The 5-years survival rate in stage IV non-small cell lung cancer (NSCLC) is $<10 \%$. Systemic therapy is advised in all stage IV patients with a good performance status. Patients with driver mutations, as an epidermal growth factor receptor (EGFR) mutated tumor or an anaplastic lymphoma kinase (ALK) rearranged tumor, can be treated with targeted therapy as tyrosine kinase inhibitors.

Prognostic as well as predictive models can be used to tailor treatment decisions by individually optimizing the balance between side effects, prognosis and quality of life of patients. Clinical variables used in prognostic models in lung cancer are for example age, gender, smoking history, performance status, histological grading and mutation status. Prognostic models can also contain biological markers that for example are measured in the blood, so called biomarkers. Image characteristics, like standardized uptake value (SUV) metrics, but also computed tomography (CT) intensity values can potentially improve prognostic and predictive models. Image biomarkers can be qualitative, so called semantic features, or quantitative, for example radiomic features. Using radiomics thousands of quantitative features can be extracted from standard-of-care medical images, like CT, PET and MRI. Radiomic features, typically divided into intensity features, shape features, texture feature and filtered features offer information about cancer phenotype.

In this thesis, the use of image features of positron emission tomography (PET) and CT imaging of stage IV NSCLC patients was investigated for prognosis assessment. Simple PET metrics were used for response assessment, CT radiomic features were used in prognostic models for overall survival and cachexia and associations between semantic features and radiomic features for prediction of the mutation status were explored. Next to that, the quality of PET imaging, the use of contrast-enhanced CT imaging and magnetic resonance (MR) imaging and the influence of image acquisition and reconstruction on radiomic features was studied. Because image biomarkers can only improve prognostic models when the imaging is acquired and reconstructed on a standardized way.

\section{Methodological challenges in image feature studies}

The first part of this thesis reviewed the image quality of contrast-enhanced (CE)-CT, magnetic resonance $(M R)$ and PET imaging in multicenter trials. When using data from multiple centers a large variability in image acquisition and reconstruction parameters exist. For image biomarker studies, it is important to use standardized imaging or to correct for variation in image parameters. The influence of image acquisition and reconstruction on radiomic feature values and stability is investigated and methods to correct this variability are proposed. The slice thickness had the largest influence on the radiomic features, but this could be reduced by resampling the images before extracting the features.

In chapter 3, image acquisition and reconstruction parameters from ${ }^{18} \mathrm{~F}-\mathrm{FDG}$ PET scans of 223 stage IV NSCLC patients included in a phase II multicenter trial were compared to recommended image parameters of the European Association of Nuclear Medicine (EANM). Only $19 \%$ of the scans had an uptake time that fulfilled the recommendations, and those scans had a smaller deviation in the mean standardized uptake value of the liver than scans with non-compliant uptake times. SUV mean of the liver can be used a reference organ due to its stable uptake over time and is therefore suitable for assessment of scan parameters. We showed that despite the agreement on a standardization protocol there was a large variability in image parameters. For future multicenter studies including PET imaging, a prospective central quality 
review during patient inclusion is needed to improve guideline compliance.

A multicenter trial, in which the added value of gadolinium MRI to dedicated CE-CT for detecting asymptomatic brain metastases was investigated in stage III NSCLC patients, showed that not only in the image acquisition and reconstruction phase differences develop but also in the starting phase of a trial (chapter 4). One hospital did not have a MRI scanner that met the required technical standard and two other hospital had to update their protocols to be able to participate in the trial. In $26 \%$ of the patients, the CE-CT of the brain did not meet the criteria for a dedicated brain scan, which was remarkably not reported. Next to all technical guidelines, we recommend that radiologists need be informed about the indication for the requested scan to determine the optimal imaging protocol and that non-imaging clinicians have the knowledge to evaluate whether the applied protocol is adequate for the requested purpose. These recommendations are expected to lead to a more uniform clinical interpretation.

Although there is a large variability in image acquisition and reconstruction parameters between different centers no effect of exposure on radiomic features was shown in chapter $\mathbf{5}$. Slice thickness did influence radiomic feature values but this could be reduced by resampling the $\mathrm{CT}$-images before feature extraction. Optimization of gray level discretization to potentially improve prognostic value can be performed without compromising feature stability.

\section{Image features for prognosis}

In the second part of this thesis the use of image features from CT and PET imaging for response assessment, prognosis, mutation status and cachexia is investigated.

In chapter 6 tumor response defined as a $30 \%$ decrease in CT and PET parameters after three weeks was compared to the response criteria in solid tumors (RECIST) response at week 6. A $30 \%$ decrease in the peak SUV value identified more patients as responders compared to a $30 \%$ decrease in CT diameter, however this was not correlated to overall survival. Although the phase II trial in which patients were randomized between paclitaxel-carboplatin-bevacizumab with or without nitroglycerin showed no added value of nitroglycerin on progression free survival, there was a significant larger decrease in CT diameter in the control group compared to the intervention group, which even shows, although not significant, negative effect of nitroglycerin. No significant difference was seen in the PET parameters between the two treatment arms. This might be due the decreased perfusion caused by bevacizumab in combination with the timing of the PET scan.

In 2014, our group published a prognostic radiomic signature for predicting overall survival. This signature was trained and validated in a cohort of stage I-III NSCLC patients. In chapter 7 we aimed to validate this prognostic radiomic signature in stage IV NSCLC patients undergoing chemotherapy. We showed that patients having a prognostic index lower than the signature median had a significantly better overall survival than patients with a prognostic index higher than that median (Hazard Ratio 1.445, $95 \%$ confidence interval 1.07-1.95, $\mathrm{p}=0.02$ ). Although the radiomic signature derived from daily practice CT scans, has prognostic value for stage IV, the signature performs less than for stage I-III.

Qualitative image characteristics, such as cavitation or air bronchogram are prognostic for treatment outcome in NSCLC. In chapter $\mathbf{8}$ we showed that in radiomic features of the primary tumor could predict the semantic features air bronchogram, necrosis and pleural effusion (area under the curve $0.71,0.68,0.65$, respectively) and that radiomic features of the ipsilateral lung are associated to pleural thickening, satellite nodules and pleural effusion (AUC 0.62, 0.64 and 0.87 , respectively).

Skeletal muscle loss is associated with low quality of life, diminished treatment efficacy and poor survival rates in cancer patients. Early prediction of patients that will suffer from cachexia may lead to timely treatment and prevention. In chapter $\mathbf{9}$ we assessed if baseline skeletal muscle radiomic features predicts future muscle loss. Although baseline radiomic features of the skeletal muscle were not predictive for muscle loss (AUC 0.49), differences in intensity and texture radiomic 
features over time were seen between patients with and without muscle loss (AUC 0.68, $95 \% \mathrm{Cl}$ 0.51-0.84).

\section{Future perspectives}

In chapter $\mathbf{1 0}$ we discussed the results presented in this thesis and the future perspectives of image features. We hypothesize that standardization of image acquisition, reconstruction and analysis will lead to less variation in image parameters and therefore more reliable models. In combination with initiatives as distributed learning in which data does not leave the hospital anymore, deep learning and machine learning prognostic models will in the future include image characteristics as well as other clinical variables that will improve patient care by prolonged survival and a better quality of life. 

Samenvatting 


\section{Achtergrond en doel van het proefschrift}

In Nederland krijgen jaarlijks 100.000 mensen de diagnose kanker, hoewel velen van hen zullen genezen, overlijden er nog steeds 40.000 patiënten. Van deze 40.000 doden door kanker is $24 \%$ te wijten aan longkanker. Het overlevingspercentage bij longkanker is afhankelijk van het stadium van de ziekte. Patiënten met uitzaaiingen op afstand worden onafhankelijk van de grootte van de tumor en het aantal aangedane lymfeklieren geclassificeerd als stadium IV. Het 5-jaarsoverlevingspercentage in stadium IV niet-kleincellig longcarcinoom patiënten is minder dan $10 \%$. Systeemtherapie wordt geadviseerd voor patiënten met stadium IV ziekte met een goede conditie. Patiënten met een zogenaamde "driver mutatie" zoals epidermale groei factor receptor gemuteerde tumor of een tumor met een translocatie in het anaplastic lymphoma kinase gen kunnen worden behandeld met gerichte therapie bestaande uit tyrosine kinase remmers.

Prognostische en predictieve modellen kunnen worden gebruikt om behandelkeuzes te sturen waardoor de balans tussen bijwerkingen, prognose en kwaliteit van leven geïndividualiseerd en geoptimaliseerd wordt. Klinische variabelen die gebruikt worden in prognostische modellen in longkanker zijn bijvoorbeeld leeftijd, geslacht, rookgeschiedenis, conditie, histologie en mutatiestatus. Prognostische modellen kunnen ook biologische merkers bevatten die bijvoorbeeld in het bloed gemeten kunnen worden, de zogenoemde bloed-biomerkers. Beeldeigenschappen, zoals de activiteit (SUV) van een positronemissietomografie (PET) scan en de intensiteit (HU) van een computertomografie (CT), kunnen potentieel prognostische en predictieve modellen verbeteren. Beeldmerkers kunnen zowel kwalitatief, zogenoemde semantische beeldeigenschappen, als kwantitatief, zogenoemde radiomics beeldeigenschappen, zijn. Door het gebruik van radiomics kunnen duizenden beeldeigenschappen berekend worden uit standaard medische beelden, zoals CT, PET en magnetische resonantie (MR) beelden. Radiomics beeldeigenschappen, te verdelen in intensiteitsbeeldeigenschappen, vormbeeldeigenschappen, textuurbeeldeigenschappen en gefilterde beeldeigenschappen, bevatten informatie over het kanker fenotype.

In dit proefschrift is het gebruik van beeldeigenschappen van PET- en CT-beelden van stadium IV niet-kleincellig longcarcinoom patiënten onderzocht met als doel het bepalen van de prognose van patiënten. Simpele maten voor PET activiteit zijn gebruikt voor responsbepaling op chemotherapie en $\mathrm{CT}$ radiomics beeldeigenschappen zijn gebruikt in prognostische modellen voor het voorspellen van overleving en cachexie, ongewild gewichtsverlies. Daarnaast zijn relaties tussen semantische beeldeigenschappen en radiomics beeldeigenschappen voor het voorspellen van de mutatiestatus onderzocht. Van belang voor deze vraagstelling is de kwaliteit van contrast-versterkte CT, MR, en PET. Dit is onderzocht in een multicentrische studie waar gekeken is naar de invloed van beeldacquisitie- en reconstructieparameters op radiomics beeldeigenschappen. Beeldeigenschappen kunnen prognostische modellen namelijk alleen verbeteren als er gebruik wordt gemaakt van gestandaardiseerde beeldacquisitie en reconstructie.

\section{Methodologische uitdagingen in beeldeigenschappen studies}

In het eerste deel van dit proefschrift is er gekeken naar de beeldkwaliteit van contrast-versterkte CT-, MR- en PET-beelden in multicentrische trials. Wanneer er data gebruikt wordt uit verschillende centra zal er een variatie in beeldacquisitie- en reconstructieparameters zijn. Voor beeldmarker studies is het belangrijk dat gestandaardiseerde beelden gebruikt worden dan wel dat er gecorrigeerd wordt voor de variatie in beeldparameters. De invloed van beeldacquisitie- en reconstructieparameters op radiomics beeldeigenschappen en hun stabiliteit is onderzocht en er is een voorstel gedaan voor correctiemethoden voor deze variatie. De plakdikte bleek de grootste invloed te hebben op de radiomics beeldeigenschappen, maar dit effect kan verminderd worden door de beelden te herschalen. 
In hoofdstuk 3 zijn beeldacquisitie- en reconstructieparameters van PET scans van 223 stadium IV niet-kleincellig longcarcinoom patiënten, die geïncludeerd waren in een fase II multicentrische studie, vergeleken met de door de Europese associatie van nucleaire geneeskunde (EANM) aanbevolen beeldparameters. Slechts $19 \%$ van de beelden had een opname tijd die voldeed aan de aanbevelingen, en deze scans hadden een kleinere variatie in de gemiddelde activiteit $\left(S U V_{\text {mean }}\right)$ in de lever dan de scans met een opname tijd die niet-conform de aanbevelingen was. De gemiddelde activiteit in de lever kan worden gebruikt als referentie doordat de activiteit stabiel is over de tijd en daardoor gebruikt kan worden om de beeldparameters te controleren. We hebben laten zien dat ondanks dat men het eens was over het beeldacquisitie- en reconstructieprotocol er in dagelijkse praktijk een grote variatie in beeldparameters is. Voor toekomstige multicentrische studies, die gebruik maken van PET-beelden, is het belangrijk dat vooraf en tijdens patiëntinclusie, een centrale kwaliteitscheck gedaan wordt om de variatie in beeldacquisitie en reconstructie te minimaliseren.

In een multicentrische studie, waar de toegevoegde waarde van gadolinium MR ten opzichte van contrast-versterkte $\mathrm{CT}$ in de detectie van asymptomatische hersenmetastasen werd onderzocht in stadium III niet-kleincellig longcarcinoom patiënten, is gebleken dat er niet alleen verschillen ontstaan in de beeldacquisitie- en reconstructie-fase, maar ook tijdens de opstartfase van een studie (hoofdstuk 4). Een van de ziekenhuizen had geen MR-scanner die voldeed aan de benodigde technische specificaties en twee andere ziekenhuizen moesten hun protocollen herzien voor ze konden deelnemen aan de studie. Bij $26 \%$ van de patiënten voldeed de contrast-versterkte CT niet aan de criteria voor een speciale hersenscan, wat opmerkelijk genoeg ook niet vermeld werd in het verslag. Naast alle technische richtlijnen raden we aan dat radiologen geïnformeerd worden over de indicatie waar de scan voor gemaakt moet worden, zodat zij kunnen meedenken over het meest optimale scan protocol. Daarnaast moeten andere specialisten inzicht hebben in de verschillende protocollen zodat ze mede kunnen evalueren of het toegepaste scan protocol adequaat is voor het aangevraagde doel. Deze aanbevelingen zouden moeten leiden tot een meer uniformer klinische interpretatie.

Hoewel er een grote variatie in beeldacquisitie- en reconstructieparameters tussen verschillende centra is, is er in hoofdstuk $\mathbf{5}$ geen effect gezien van de exposie op radiomics beeldeigenschapwaarden. De plakdikte beïnvloedde de radiomics beeldeigenschapwaarden wel, maar dit kan verminderd worden door de CT-beelden eerst te herschalen voordat de beeldeigenschappen uitgerekend worden. Optimalisatie van grijswaardendiscretisatie, om potentieel de prognostische waarde van radiomics beeldeigenschappen te verbeteren, kan gedaan worden zonder de stabiliteit te veranderen.

\section{Beeldeigenschappen voor prognose}

In het tweede deel van dit proefschrift wordt het gebruik van beeldeigenschappen van CT- en PET-beelden voor response beoordeling, prognose, mutatiestatus en cachexie beschreven.

In hoofdstuk 6 is tumor respons, gedefinieerd als een afname van $30 \%$ in de CT- en PETparameters na drie weken, vergeleken met de respons criteria in solide tumoren (RECIST) na zes weken. Een afname van $30 \%$ in de piek activiteit $\left(S_{U} U V_{\text {peak }}\right)$ identificeerde meer patiënten als responders dan een $30 \%$ afname in de CT-diameter, alhoewel dit niet gecorreleerd is aan overleving. Hoewel de fase II studie, waar patiënten gerandomiseerd waren tussen paclitaxelcarboplatin-bevacizumab met of zonder nitroglycerine, geen toegevoegde waarde liet zien van nitroglycerine op de progressie vrije overleving, was er een significant grotere afname in CTdiameter in de controlegroep. Er was geen significant verschil in PET-parameters tussen beide groepen. Dit zou kunnen komen door de verstoorde perfusie, veroorzaakt door bevacizumab, in combinatie met de timing van de scan.

In 2014 heeft onze groep een prognostisch radiomics profiel voor overleving gepubliceerd. Dit profiel is getraind en gevalideerd in een cohort van stadium I-III niet-kleincellig longcarcinoom patiënten. In hoofdstuk 7 hebben we geprobeerd dit prognostisch radiomics 
profiel te valideren in stadium IV niet-kleincellig longcarcinoom patiënten die behandeld werden met chemotherapie. We hebben laten zien dat patiënten met een prognostische index lager dan de gemiddelde prognostische index een significant betere overleving hadden dan patiënten met een hoge prognostische index (Hazard Ratio 1.445, $95 \%$ betrouwbaarheidsinterval 1.07-1.95, $\mathrm{p}=0.02$ ). Hoewel het radiomics profiel afgeleid van standaard CT-beelden ook prognostische waarde heeft in stadium IV, voorspelt het profiel beter in stadium I-III.

Kwalitatieve beeldeigenschappen, zoals cavitatie of luchtbronchogram, zijn prognostisch voor behandeluitkomsten bij het niet-kleincellig longcarcinoom. In hoofdstuk $\mathbf{8}$ hebben we laten zien dat radiomics beeldeigenschappen van de primaire tumor de semantische beeldeigenschappen luchtbronchogram, necrose en pleurale effusie kunnen voorspellen (AUC 0.71, 0.68, 0.65, respectievelijk) en dat radiomics beeldeigenschappen van de ipsilaterale long gerelateerd zijn aan pleurale verdikking, satelliet laesies en pleurale effusie (AUC 0.62, 0.64 en 0.87, respectievelijk).

Skeletspierverlies is gerelateerd met een lagere kwaliteit van leven, een slechtere behandeluitkomst en een slechtere prognose in kankerpatiënten. Een vroege voorspelling van cachexie kan leiden tot preventie en eerdere behandeling. In hoofdstuk 9 hebben we geïnventariseerd of radiomics beeldeigenschappen van de skeletspier op de CT, gemaakt voor de start van behandeling, toekomstig spierverlies kan voorspellen. Hoewel deze radiomics beeldeigenschappen niet voorspellend waren voor spierverlies (AUC 0.49), waren er verschillen in radiomics intensiteitseigenschappen en textuureigenschappen over de tijd te zien tussen patiënten met en zonder spierverlies (AUC 0.68, $95 \%$ betrouwbaarheidsinterval 0.51-0.84).

\section{Toekomstperspectieven}

In hoofdstuk $\mathbf{1 0}$ hebben we resultaten en toekomstperspectieven van de studies over beeldeigenschappen in dit proefschrift besproken. We denken dat gestandaardiseerde beeldacquisitie, reconstructie, analyse en initiatieven als "distributed learning" , waarbij data het ziekenhuis niet meer hoeft te verlaten, in combinatie met "deep learning" en "machine learning" in de toekomst zal leiden tot prognostische modellen die naast klinische variabelen ook beeldeigenschappen bevatten de patiëntzorg zal verbeteren door langere overleving en een betere kwaliteit van leven. 

Valorisation addendum 


\section{Prognostic models}

Although there have been innovations in treatment options for stage IV non-small cell lung cancer, the overall survival rate for these patients is still low. Innovations in treatment consist for example of specific drugs that are optimized for various cancer types, e.g. EGFR mutated tumors treated using tyrosine kinase inhibitors. However, this treatment is not effective in, for example, patients with a KRAS mutation. And if patients do not respond on a certain treatment, quality of life of these patients can be improved by stopping the treatment. In the first part of this thesis, prognostic models including image biomarkers are developed and validated.

Prognostic models help in selecting patients that benefit from certain therapies by which side effects are reduced and quality of life is improved. If a tumor is not responding on a specific chemotherapy, another treatment may be used which is potentially more effective. Prognostic models already are able to predict the chance that a patient will respond on the treatment before the start of treatment. We showed that PET-based response assessment showed response already after three weeks of treatment, which is earlier than CT-based response assessment usually performed after 6 weeks of treatment. Early response assessment and prognostic models will lead to economically benefits through less side effects, less expensive treatments and better prognosis. Additionally cost effectiveness analyses should be performed in which the relative value of a specific treatment is measured as the additional cost to achieve an incremental health benefit. Information from prognostic and/or predictive models can be used as input for cost effectiveness analyses to predict survival or side effects, by which life expectancy adjusted for morbidity or quality of life for different treatments can be compared.

Next to that, prognostic models can be integrated in decision support systems. Decision support systems help patients together with their physician to choose between different treatments, based on results of previous studies and personal preferences. For some patients, quality of life is more important while others prefer a prolonged survival. On www. predictcancer.org prognostic models for overall survival, local recurrence, cost effectiveness and side effects like dysphagia can be found for brain metastases, endometrium cancer, head and neck cancer, lung cancer, esophageal cancer, prostate cancer and rectum cancer. With initiatives like predictcancer.org decision support systems may easily be implemented in the clinic.

\section{Image features}

Imaging is an important part of routine care in diagnosis, treatment and follow up in oncology. Image biomarkers improve prognostic models in a non-invasive way. Image biomarkers can be qualitative, so called semantic features, but also quantitative, so called radiomic features. While scoring semantic features is time consuming and inter observer dependent, radiomic features are automatically extracted on repeated imaging. Imaging is non-invasive and widely available because it is used in standard clinical care. In chapter 8 we showed that there are associations between semantic features and radiomic features that are correlated to mutation status of the tumor. When imaging is used to determine the mutation status of tumors, invasive biopsies that are only samples of mostly heterogeneous tumors can be reduced or in the future are not needed anymore.

Many publications already showed the potential of radiomics. Radiomic signatures can often be used in different tumor sites. Software developments are ongoing and more and more open source radiomic packages become available. By making radiomic software open source, usage by more research departments makes external validation of radiomic signatures much easier. Next to that, initiatives are ongoing in which radiomics is implemented in clinical software. An example of this is the collaboration between OncoRadiomics and AQUILAB in the ARTIVIEW software package. Such software could be used in either clinical studies, to develop new predictive signatures or in clinical decision support systems based on defined signatures. These commercial 
software packages consist of a database system in which multimodality images could be saved, fused or registered, segmented and used for dose calculations. By implementing radiomics in already clinical used software packages, radiomics can easily be used in diagnosis, segmentation and response assessment. External validation studies validated existing radiomic signatures in different tumor sites as well as using different image settings. Radiomic signatures that are externally validated in large datasets will lead to useful clinical products.

Radiomics may also be very useful in diagnosis. In chapter 9 we showed that differences in delta texture radiomic features over time might reflect myosteatosis. This shows that using radiomics biological processes behind some diseases, for example cachexia can be unraveled. This can in the future possibly lead to new treatment options. An increasing number of studies is looking into radiomics for differentiating benign lesions and malignant lesions in for example breast, prostate and lung. Using this information leads to earlier diagnosis and therefore a prolonged survival and better quality of life.

The most time consuming step in the radiomics process is still segmentation of the region of interest. Machine learning, which is a subset of artificial intelligence to automatically detect patterns in data, by which segmentation is automated, combined with radiomics, will in the future automatically analyze large datasets used for prognostic or diagnostic models.

\section{Image quality}

Although for automatic extracted features it is important that the images used are acquired on a standardized way. In the second part of the thesis image quality in multicenter trials is reviewed and from that recommendations are formed to improve image quality in future multicenter trails. When using images from multiple centers there is a large variation in image acquisition and reconstruction settings, which influence radiomic feature values and feature stability and therefore results from prognostic models including radiomic features. In chapter 5 we showed that slice thickness influences radiomic features, however this can be reduced by resampling the images prior to feature extraction. Studies investigating the influence of scan parameters on radiomic features will lead to a more standardized method for imaging in radiomic studies and therefore in less variation in radiomic features and more reliable radiomic signatures.

Although guidelines for PET imaging in multicenter trials exist, in chapter 3 we showed that there is still a large variability in image acquisition and reconstruction parameters between Dutch institutes, which is probably even larger between European centers and centers worldwide. The largest difference was seen in the time between FDG injection and the start of the PET scan. Longer uptake times will lead to higher SUV values, which for response assessment can lead to a false response assessment. When using PET scans from multiple centers in response assessment studies we recommend to use a prospective central quality review to reduce the variability between institutes and to better comply with the existing guidelines, which leads to a better overall image quality, higher reproducibility and less inter-center variation.

For contrast-enhanced (CE-)CT in diagnosing asymptomatic brain metastases in non-small cell lung cancer no clear guidelines exist. CE-CT scans with the arms of the patients next to the head are often reported to be diagnostic, while the arms of the patients induce beam hardening which influences the image quality. In chapter 4 we showed that it is important that clinicians inform imaging experts about the indication of a specific scan to help radiologists choose the optimal scan protocol and to check the image quality for the requested purpose. This will lead to a more uniform interpretation, less variation in multicenter trials and uniform treatment decisions; all improving treatment quality. 

Dankwoord 
$\mathrm{Na}$ ruim vier jaar is dan het eindelijk tijd het laatste hoofdstuk van mijn proefschrift te schrijven. Het laatste hoofdstuk is wat mij betreft niet het makkelijkste hoofdstuk, maar wel leuk om te schrijven omdat ik nu pas besef hoeveel mensen bijgedragen hebben aan de leuke jaren van onderzoek doen.

Allereerst wil ik graag mijn promotoren prof. dr. Philippe Lambin en prof. dr. Anne-Marie Dingemans bedanken voor het faciliteren van mijn onderzoek. Philippe, bedankt dat ik deel uit mocht maken van een wisselende internationale multidisciplinaire onderzoeksgroep die voor mij begonnen is bij MAASTRO Clinic en welke ik verlaten heb als the D-Lab onderdeel van Universiteit Maastricht. Ik bewonder uw kwaliteit om op de hoogte te zijn van de grote lijnen van alle onderzoeken van uw PhD-studenten en het inschattingsvermogen in hoe het onderzoek het beste gepubliceerd kan worden. Anne-Marie, doordat onze achtergronden veel verschilden, heb ik heel veel geleerd over de klinische aspecten van longkanker. Bedankt voor het zeer kritisch lezen en becommentariëren van mijn manuscripten, ze zijn er echt beter van geworden. Daarnaast wil ik graag mijn copromotor dr. ir. Wouter van Elmpt bedanken. Wouter, wat was het ontzettend fijn dat ik altijd bij je binnen kon lopen en dat je altijd snel reageert op mail, of het nu inhoudelijk of meer praktisch was, je hebt altijd een antwoord klaar. Het was ook erg fijn iemand met een technische achtergrond te hebben in een team wat verder vooral uit clinici bestond. Bedankt voor je tijd en steun, ook in de projecten die je zelf misschien minder interessant vond en heel veel succes met de nieuwe uitdagingen in het Physics Innovation Team. Ook wil ik graag prof. dr. Esther Troost bedanken voor de begeleiding en het controleren van vele intekeningen in het begin van mijn promotietraject.

Graag wil ik de leden van de beoordelingscommissie bedanken (prof. dr. F.M. Mottaghy, prof. dr. J.G.J.V. Aerts, prof. dr. W.H. Backes, prof. dr. M. van den Heuvel en dr. A. Hoeben) voor het kritisch lezen en beoordelen van mijn proefschrift.

De data gebruikt in dit proefschrift maakt deel uit van klinische studies. Dergelijke studies zijn enkel mogelijk door de bereidheid en het vertrouwen van patiënten om hier aan deel te nemen. Bedankt voor het participeren in de studies en voor het geven van toestemming om de data te gebruiken voor wetenschappelijk onderzoek. Ook wil ik hier graag Vincent van der Noort bedanken voor het datamanagement van de NVALT12 studie. En de medewerkers van de ICT/servicedesk van MAASTRO Clinic voor het oplossen van alle computerproblemen en het verzamelen van data uit andere ziekenhuizen. Daarnaast wil ik ook graag Floor bedanken voor het zorg dragen van de data transfer agreements en algemene onderzoekssupport. En Rianne voor het inplannen van afspraken met Philippe, maar ook voor alle organisatie rondom mijn promotie.

Tijdens het begin van mijn promotietraject hebben we in het kader van de NVALT12 studie vele teleconferenties gehad met experts uit verschillende deelnemende ziekenhuizen (prof. dr. O.S. Hoekstra, prof. dr. E.F. Smit, prof. dr. H.J.M. Groen en prof. dr. R. Boellaard), bedankt voor de nuttige discussies en voor het kritisch meelezen van de manuscripten. Next to the Dutch collaborators, I would also like to thank dr. Stefania Rizzo and colleagues from Milan for the fruitful collaboration in the EGFR/KRAS/ALK mutation projects.

Tijdens mijn HBO studie Technische Natuurkunde heb ik stage gelopen bij de radiotherapie afdeling RISO in Deventer. Daar is mijn interesse in de klinische fysica bij de radiotherapie begonnen, wat ook een aanleiding is geweest voor mijn promotieonderzoek, bedankt Rik. Andre en Guido. Deze interesse is verder aangewakkerd op de klifio kamer (Ellen, Leah, Hanneke, Petra en Rob) in Utrecht waar ik mijn afstudeerproject gedaan heb onder begeleiding van Bas en Jochem, bedankt allemaal. Tijdens het 100 -uren project bij MAASTRO heb ik veel geleerd over dose-guided radiotherapie wat tijdens mijn klifioperiode vast nog van pas komt, bedankt Bas, Lucas en Esther. Ook wil ik graag mijn nieuwe collega's uit het CZE (Paul, Coen, Danny, Hanneke, Jacco en Wim) bedanken voor de kans om mijn promotie af te maken tijdens het opleidingstraject tot klinisch fysicus. En ook mijn medeklifio's uit het CZE (Esther en Kathrin), maar ook uit Maastricht (Esther en Celine) voor het delen van ervaringen en aanhoren van promotiefrustaties.

Bedankt voor de leerzame longkanker research meetings (Janna, Jules, Juliette, Karin en Lizza). Lizza ook heel erg bedankt voor het controleren van vele intekeningen, de fijne samenwerking bij het paper over imaging voor medici en de uitwisseling van medische informatie 
vanuit jouw kant en meer technische informatie vanuit mijn kant. Karin bedankt voor de gezelligheid tijdens onze koffiedates, de samenwerking bij de cachexieprojecten en de heerlijke etentjes. Heel veel succes met het afronden van je eigen promotie en daarna natuurlijk met de opleiding tot longarts. Ook wil ik graag prof. dr. A.M.W.J. Schols bedanken voor de samenwerking bij het cachexie paper. Bart bedankt voor het controleren van alle intekeningen, $i k$ heb er veel van geleerd.

Although I didn't had a direct collaboration with anyone of MAASTRO-Lab I would like to thank you all for the nice meetings, wintersport and ESTRO (Ludwig, Ala, Nicolle, Tessa, Marijke, Carolien, Raymond, Kranthi, Venus en Damiënne).

In the research group of Philippe, in the beginning at MAASTRO Clinic, later at the UM as the D-Lab, I shared many happy moments during coffee-breaks, "vlaai" moments, drinks, lunchwalks, diners, carnaval and sports, thank you all (Abdalla, Abir, Adriana, Alberto, Ana, Anshu, Bregtje, Brent, Cary, Celine, Claire, Daan, Daniela, Davide, Emmanuel, Ester, Esther, Frank, Gabriel, Georgi, Giacomo, Guangyao, Isabel, Inge (ook bedankt voor alle klinische ervaring), Iva, Jane, Jean, Joana, Johan (ook bedankt voor de ICT support), Jose, Leon, Leonard, Lotte, Lucas, Manon, Mark, Marta, Mathieu, Matilde, Mariana, Murillo, Patrick, Qing, Pedro, Raghu, Samir, Scott, Sebastian, Sergey, Simon, Stefan, Turkey, Yvonka, Zhen, Zhenwei). Ook wil ik graag de studenten bedanken die ik tijdens mijn promotie heb mogen begeleiden, Stefan bedankt voor de fijne samenwerking, Esma ook bedankt voor de fijne samenwerking en heel veel succes verder met je stage en het afronden je studie. Shane I would also like to thank you for supervising me during my internship, you're a good teacher, it is also because of you that I started my PhD. We still miss your interesting conversations during lunch and coffee breaks. Sara thank you for all the help with radiomics, you're a great person to work with and a nice person to have around. Skadi, naast dat we een interesse delen en daardoor nu allebei klinisch fysicus in opleiding zijn, hebben we ook een aantal karaktertrekken gemeen, bedankt dat ik jouw rol als detective mocht overnemen. Karen, de moeder van de groep, het was fijn dat er altijd iemand was om je verhaal mee te delen, bedankt. Ralph bedankt voor alle hulp met radiomics, elke keer als de software werkte wist ik toch weer een patiënt te vinden waar het niet bij lukte. En natuurlijk ook bedankt voor alle lunch wandelingen, je was toch wel de meest stabiele factor in het wandelteam. Arthur, gedurende mijn hele promotietraject kon ik bij jou terecht voor alle vragen met betrekking tot statistiek, heel erg bedankt hiervoor en ook bedankt voor de begeleiding bij de laatste papers. Henry, although we didn't collaborate on any projects, your door was always open and even more important thank you for all the nice climbing hours where we discussed more than climbing techniques. Sean, my work spouse, although I think I probably helped you more than you me, it was nice to have a native English senior researcher next to me for a couple of years. I wish you, Celine and Luana the best in life. Timo, the German that speaks better Dutch than most Dutch students, thank you for the collaboration in the semantic feature paper and the cachexia paper. It is very nice working with someone that is structured, realistic and funny, good luck finishing your own PhD. Aniek, bedankt voor je hulp met Matlab, maar vooral voor de gezelligheid als buurvrouw, maar ook tijdens het sporten, de borrels, carnaval, de ladiesnights en de etentjes. Jurgen, wat hebben we veel Belgische woorden en grappen geleerd van jou. Bedankt hiervoor en ook bedankt voor de gezelligheid en voor je kennis met betrekking tot MRI. Cecile, Relinde en Janita, met z'n drieën/vieren zijn wij Maastrio/Maasquatro en hebben we regelmatig gezellig met elkaar gegeten, vooral aan het einde van mijn promotie. Door de stage van Janita in Odense hebben we nu het jaarlijks weekendje weg geïntroduceerd. Ik wens jullie allen veel succes met het afronden van jullie promoties en hoop dat we de gezellige etentjes en weekendjes er gewoon inhouden. Relinde ook bedankt voor het carpoolen van Eindhoven naar Maastricht, was erg fijn dat er af en toe iemand anders terugrijdt en bedankt voor de fijne gesprekken.

Mijn paranimfen Janita en Ruben, ik ben ontzettend blij dat jullie op deze belangrijke dag achter mij staan en met mij meedenken. Ruben, bijna tegelijk begonnen en nu bijna tegelijk gepromoveerd. Het was erg fijn iemand te hebben die hetzelfde proces doorloopt. Bedankt voor de introductie van carnaval, wat natuurlijk al begint op de $11 \mathrm{de}$ van de $11 \mathrm{de}$, de sportieve uurtjes bij clubpower en squash en de gezellige autoritjes. Na de moleculaire beeldvormingscursus in 
Madrid, waar we samen naar toe geweest zijn, zijn we naast (bijna)buren ook vrienden geworden. Ook heel erg bedankt voor het op ons huis, huisdieren en planten passen tijdens onze vakanties. Ik hoop dat we ondanks de afstand en de nieuwe drukke banen vrienden zullen blijven. Janita, vanaf jouw eerste werkdag had ik mijn werkmaatje gevonden, dit is inmiddels veranderd naar een hele goede vriendin. Voor alle werk gerelateerde vragen waren we mekaars vraagbaak, daarnaast kon je me altijd helpen de code (Matlab, R) aan de praat te krijgen. Samen zijn we naar de ASTRO geweest en hebben we daarnaast twee hele leuke weken vakantie gevierd in Amerika, ik zal die walvissen nooit vergeten. Ook hebben we samen veel gesport, clubpoweren met Ruben, Aniek, Jurgen, Cecile en Shane, squash cursus, klimmen samen met Henry en Ciska, of de tenniscursussen in de winter waar het elke week spannend was of het doorging. Sinds mijn verhuizing hebben we er ook allebei een logeeradresje bij. Ik wens je heel veel succes met het afronden van jouw promotie en daarna heel veel succes en plezier in Australië, maar wel terugkomen hoor.

Tijdens een promotietraject is ontspanning ook erg belangrijk. Dr. ir. Dennis Bakhuis (en Kim), Jeroen (en Emilie en Arthur), Philip, Thomas A., Thomas W. (en Michelle) en Vincent bedankt voor de gezellige borrels, etentjes, housewarmings en feestjes. Verschillende vooropleidingen kwam samen in een schakelprogramma voor Biomedische Technologie, na verschillende masters zijn er vier promotietrajecten uitgerold bij vier verschillende universiteiten. Ik heb het heel waardevol gevonden te zien welke verschillen er zijn tussen de verschillende universiteiten. Bedankt voor de gezellige etentjes, bruiloft, en promotiefeestjes (Helen, Karlijn, Nikita, Simone en Willem) en succes met afronden Helen en Willem. Miriam en Franciska de meiden van natuurkunde, bedankt voor de gezellige etentjes. Jolanda, we hebben mekaar leren kennen bij MAASTRO, jij studeerde daar af en ik liep daar stage. Doordat we allebei in regio Eindhoven woonden hebben we vaak samen bij elkaar in de trein gezeten en veel goede gesprekken gevoerd. Vervolgens kwamen we elkaar weer tegen in Utrecht, jij als promovendus, ik als afstudeerder. Nu zijn we allebei gepromoveerd en komen we elkaar mogelijk weer tegen als klinisch fysicus. Bedankt voor de lekkere etentjes en veel succes met het vinden van jouw droombaan. Harriët en Marieke, mijn vriendinnen in het Noorden, ondanks dat jullie wellicht het spoor bijster zijn wat ik eigenlijk doe, kan ik wel altijd mijn verhaal kwijt bij jullie. Bedankt voor het begrip dat ik de laatste tijd niet zoveel tijd had om langs te komen en bedankt voor de gezelligheid.

Pappa Erik en Mamma Joostien ik wil jullie graag bedanken voor de steun, er werd nooit gevraagd wanneer het af was, maar ik kon wel altijd mijn verhaal doen waar jullie altijd geïnteresseerd naar geluisterd hebben. Floris en Beata; Rosalinde, Herman, Quinten en Pepijn; Laurens, Marion, Myrthe en Mats; Liselore, Andre en Sven; Charlotte en Jane en Vincent, bedankt voor de afleiding van mijn onderzoek tijdens de afgelopen vier jaar tijdens verjaardagen. Ook wil ik graag tante Annie bedanken voor de steun en interesse.

Heit en Mem, wa hie dat ea tocht in doktor yn de famylje. Ik wol jim graach betankje foar de stipe, mei it begryp en de ynteresse. Jullie hebben me altijd gesteund en de kans gegeven te studeren, bedankt hiervoor. Ik hoop dat jullie net zo van deze dag genieten als ik, echt iets om trots op te zijn.

En last but not least Mathijs. Inmiddels zijn we al 11 jaar samen, en jij kent me dan ook als geen ander. Zonder jou was ik nooit zover gekomen. We hebben elkaar leren kennen tijdens het begin van mijn lange journey tot klinisch fysicus. Het is ooit begonnen als bijles bij wiskunde, maar je bent in heel veel zaken mijn betere helft. Samen doorstuderen aan de universiteit, we hebben elkaar er doorheen gesleept en tijdens mijn promotie stond je altijd achter me. Altijd stond het eten op tafel als ik laat thuiskwam na het werken, maar ook na het sporten of borrelen met collega's. Tijdens het afronden van mijn promotie, tevens de start van mijn nieuwe baan als klinisch fysicus in opleiding kochten wij ons eerste huis. Jij gaf mij de ruimte mijn promotie af te ronden en aan mijn nieuwe baan te wennen en probeerde tevens het in huis zo makkelijk mogelijk te maken voor mij. En natuurlijk ook bedankt voor het helpen met de layout van mijn boekje, nu is het toch echt ons boekje geworden. Mijn excuses voor alle stress en het gezeur te laatste tijd, daar is vanaf nu geen reden meer toe. Bedankt dat je er altijd voor me bent, ik hou van je. 

Curriculum vitae 
Evelyn de Jong was born on the 24th of July 1987 in Drachten, the Netherlands. After finishing her secondary education at the Drachtster Lyceum in Drachten in 2004, she started with the Bachelor Medisch Beeldvormende en Radiotherapeutische Technieken at the Hanzehogeschool in Groningen. She performed her first internship at the Radiotherapy department of the Medisch Spectrum Twente in Enschede, where she was a radiation therapist in training. After her internship she decided to switch to the Bachelor Applied Physics at Saxion Hogeschool in Enschede. During this bachelor she did an internship at the Radiotherapeutic Institute RISO in Deventer, where she investigated absolute film dosimetry for delivery quality assurance for tomotherapy. The other internship was done at the KVI-Center for Advanced Radiation Technology in Groningen, where she studied the dose response of radiochromic film to Carbon ions. For her graduation project, which Evelyn performed in the NIM-biomagnetism group of the University of Twente, she analyzed, balanced, tested and improved a new 4K SQUID magnetometer, which was designed to determine the magnetic relaxation behavior of nanoparticles (e.g. iron filled carbon nanotubes) in suspension, at low frequencies. After her graduation in 2011, she decided to continue studying and enrolled in the premaster of Biomedical Engineering at the Eindhoven University of Technology in Eindhoven. During the master Medical Engineering, she spent three months at the radiotherapy institute MAASTRO Clinic in Maastricht, where she worked on the effect of different tissue modelling schemes on the dose distribution in low energy breast brachytherapy and the influence of adjustment of the treatment plan based on the cone-beam CT for lung cancer patients. For her Master thesis, she did a nine months project at the radiotherapy department of the University Medical Center in Utrecht, where she studied small field dosimetry in a magnetic field. In 2014 she obtained her Master's degree. In August of the same year, she joined the research team of Maastricht University. As a $\mathrm{PhD}$ candidate she investigated the prognostic value of a variety of image features of CT and PET images of stage IV non-small cell lung cancer patients. During her work, she found out that there are many methodological challenges in image feature studies, which she studied further by which she was able to provide recommendations for future multicenter trials with respect to imaging. In April 2018, she started as a Medical Physicist in training at the Catharina hospital in Eindhoven.

\section{Grants}

- BIGART travel grant, Biology-Guided Adaptive Radiotherapy, 2017, Aarhus, Denmark.

- NRS young investigator travel grant, Annual Meeting of the American Society for Radiation Oncology, 2016, Boston, US.

- NRS young investigator travel grant, ESTRO/EANM course on Molecular Imaging and Radiation Oncology, 2015, Madrid, Spain. 


\section{Scientific publications}

- Lambin P., Zindler J., Vanneste B.G.L., Van De Voorde L., Jacobs M., Eekers D., Peerlings J., Reymen B., Larue R.T.H.M., Deist T.M., de Jong E.E.C., Even A.J.G., Berlanga A.J., Roelofs E., Cheng Q., Carvalho S., Leijenaar R.T.H., Zegers C.M.L., van Limbergen E.J., Berbee M., van Elmpt W., Oberije C., Houben R., Dekker A., Boersma L.J., Verhaegen F., Bosmans G., Hoebers F., Smits K.M. \& Walsh S. Modern clinical research: How rapid learning health care and cohort multiple randomised clinical trials complement traditional evidence based medicine. Acta Oncol. 2015; 54(9):1289-300.

- White S.A., Reniers B., de Jong E.E.C., Rusch T. \& Verhaegen F. A comparison of the relative biological effectiveness of low energy electronic brachytherapy sources in breast tissue: a Monte Carlo study. Phys Med Biol. 2016; 61 (1):383-99.

- Scrivener M.*, de Jong E.E.C.*, van Timmeren J.E.*, Pieters T., Ghaye B. \& Geets X. Radiomics applied to lung cancer: a review. Transl Cancer Res. 2016; 5(4):389-409.

- Lambin P., Zindler J., Vanneste B.G.L., Van De Voorde L., Eekers D., Compter I., Panth K.M., Peerlings J., Larue R.T.H.M., Deist T.M., Jochems A., Lustberg T., van Soest J., de Jong E.E.C., Even A.J.G., Reymen B., Rekers N., van Gisbergen M., Roelofs E., Carvalho S., Leijenaar R.T.H., Zegers C.M.L., Jacobs M., van Timmeren J.E., Brouwers P., Lal J.A., Dubois L., Yaromina A., van Limbergen E.J., Berbee M., van Elmpt W., Oberije C., Ramaekers B., Dekker A., Boersma L.J., Hoebers F., Smits K.M., Berlanga A.J. \& Walsh S. Decision support systems for personalized and participative radiation oncology. Adv Drug Deliv Rev. 2017; 109:131-153.

- de Jong E.E.C., van Elmpt W., Leijenaar R.T.H., Hoekstra O.S., Groen H.J.M., Smit E.F., Boellaard R., van der Noort V., Troost E.G.C., Lambin P. \& Dingemans A.-M.C. [18F]FDG $P E T / C T$-based response assessment of stage IV non-small cell lung cancer treated with paclitaxel-carboplatin-bevacizumab with or without nitroglycerin patches. Eur J Nucl Med Mol Imaging 2017; 44(1):8-16.

- Leijenaar R.T.H., de Jong E.E.C., Larue R.T.H.M., van Timmeren J.E. \& Lambin P. Radiomics: de toekomst in medische beeldvorming. Ned Tijdsch Oncol. 2017; 14:82-9.

- de Jong E.E.C., van Elmpt W., Hoekstra O.S., Groen H.J.M., Smit E.F., Boellaard R., Lambin P. \& Dingemans A.-M.C. Quality assessment of positron emission tomography scans: recommendations for future multicenter trials. Acta Oncol. 2017; 56(11):14591464.

- Larue R.T.H.M.*, van Timmeren J.E.*, de Jong E.E.C.*, Feliciani G., Leijenaar R.T.H., Schreurs W.M.J., Sosef M.N., Raat F.H.J.P., van der Zande F.H.R., Das M., van Elmpt W. \& Lambin P. Influence of gray level discretization on radiomic feature stability for different CT scanners, tube currents and slice thicknesses: a comprehensive phantom study. Acta Oncol. 2017; 56(11):1544-1553.

- Lambin P., Leijenaar R.T.H., Deist T.M., Peerlings J., de Jong E.E.C., van Timmeren J.E., Sanduleanu S., Larue R.T.H.M., Even A.J.G., Jochems A., van Wijk Y., Woodruff H., van Soest J., Lustberg T., Roelofs E., van Elmpt W., Dekker A., Mottaghy F.M., Wildberger J.E. \& Walsh S. Radiomics: the bridge between medical imaging and personalized medicine. Nat Rev Clin Oncol. 2017; 14(12):749-762.

- Sanduleanu S.*, Woodruff H.C.*, de Jong E.E.C., van Timmeren J.E., Jochems A., Dubois L. \& Lambin P. Tracking tumor biology with radiomics: A systematic review utilizing a radiomics quality score. Radiother Oncol. 2018; 127(3):349-360.

- de Jong E.E.C.*, Hendriks L.E.L.*, van Elmpt W., Gietema H.A., Hofman P.A.M., De Ruysscher D.K.M. \& Dingemans A.-M.C. What you see is (not) what you get: tools for a non-radiologist to evaluate image quality in lung cancer. Lung Cancer 2018; 123:112-115. 
- de Jong E.E.C., van Elmpt W., Rizzo S., Colarieti A., Spitaleri G., Leijenaar R.T.H., Jochems A., Hendriks L.E.L., Troost E.G.C., Reymen B., Dingemans A.-M.C. \& Lambin P. Applicability of a prognostic CT-based radiomic signature model trained on stage I-III non-small cell lung cancer in stage IV non-small cell lung cancer. Lung Cancer 2018; 124:6-11.

- Rizzo S., Raimondi S., de Jong E.E.C., van Elmpt W., De Piano F., Bagnardi V., Jochems A., Bellomi M., Dingemans A.-M.C. \& Lambin P. Genomics of non-small cell lung cancer (NSCLC): association between CT-based imaging features and EGFR and K-RAS mutations in 122 patients-an external validation. Eur J Radiol. 2018. Article in press.

- Degens J.H.R.J.*, Sanders K.J.C.*, de Jong E.E.C., Groen H.J.M., Smit E.F., Aerts J.G., Schols A.M.W.J. \& Dingemans A.-M.C. Early loss of skeletal muscle and intramuscular fat during chemotherapy is predictive for poor survival in metastatic non-small cell lung cancer. Submitted work.

- Walsh S., de Jong E.E.C., van Timmeren J.E., Ibrahim A., Compter I., Peerlings J., Sanduleanu S., Refaee T.A.G., Keek S., Larue R.T.H.M., van Wijk Y., Even A.J.G., Jochems A., Barakat M.S., Leijenaar R.T.H. \& Lambin P. Decision Support Systems in Oncology. Submitted work.

- de Jong E.E.C., Deist T.M., van Elmpt W., Jochems A., Rizzo S., Petrella F., Bellomi M., Leijenaar R.T.H., Refaee T.A.G., Hendriks L.E.L., Reymen B., Dingemans A.-M.C. \& Lambin P. Can quantitative radiomic features describe qualitative semantic features in non-small cell lung cancer patients? In preparation.

- de Jong E.E.C., Sanders K.J.C., Deist T.M., van Elmpt W., Jochems A., van Timmeren J.E., Leijenaar R.T.H., Degens J.H.R.J., Schols A.M.W.J., Dingemans A.-M.C. \& Lambin P. Radiomics approach to predict skeletal muscle response to chemotherapy in stage IV NSCLC. In preparation.

*equal contribution 